Portland State University

PDXScholar

$1-1-2011$

\title{
Student Approaches to Combinatorial Enumeration: The Role of Set-Oriented Thinking
}

Elise Nicole Lockwood

Portland State University

Follow this and additional works at: https://pdxscholar.library.pdx.edu/open_access_etds Let us know how access to this document benefits you.

\section{Recommended Citation}

Lockwood, Elise Nicole, "Student Approaches to Combinatorial Enumeration: The Role of Set-Oriented Thinking" (2011). Dissertations and Theses. Paper 338.

https://doi.org/10.15760/etd.338

This Dissertation is brought to you for free and open access. It has been accepted for inclusion in Dissertations and Theses by an authorized administrator of PDXScholar. Please contact us if we can make this document more accessible: pdxscholar@pdx.edu. 
Student Approaches to Combinatorial Enumeration:

The Role of Set-Oriented Thinking

by

Elise Nicole Lockwood

A dissertation submitted in partial fulfillment of the requirements for the degree of

\author{
Doctor of Philosophy \\ in \\ Mathematics Education
}

Dissertation Committee:

Sean Larsen, Chair

Cynthia Brown

John Caughman

Karen Marrongelle

Jennifer Noll

Portland State University

(C)2011 
Lockwood Dissertation - i

\begin{abstract}
Combinatorics is a growing topic in mathematics with widespread applications in a variety of fields. Because of this, it has become increasingly prominent in both K-12 and undergraduate curricula. There is a clear need in mathematics education for studies that address cognitive and pedagogical issues surrounding combinatorics, particularly related to students' conceptions of combinatorial ideas. In this study, I describe my investigation of students' thinking as it relates to counting problems. I interviewed a number of postsecondary students as they solved a variety of combinatorial tasks, and through the analysis of this data I defined and elaborated a construct that I call set-oriented thinking. I describe and categorize ways in which students used set-oriented thinking in their counting, and I put forth a model for relationships between the formulas/expressions, the counting processes, and the sets of outcomes that are involved in students' counting activity.
\end{abstract}


Lockwood Dissertation - ii

\section{Acknowledgments}

The completion of my dissertation and my doctoral degree would not be possible without the help of many professors, colleagues, friends, and family members, and I take a moment now to acknowledge a handful of these people by name.

I first thank the members of my dissertation committee. My advisor, Dr. Sean Larsen, provided valuable insight and feedback that greatly improved the quality of the study and the final document. Even more, he has always been a supportive advocate on behalf of his graduate students; I am very grateful for his continued guidance throughout my time in the program. I also thank Dr. Karen Marrongelle, who never failed to meet with me and help me think through a particular aspect of my study. She, too, played a vital role throughout my entire program, contributing greatly to my development as a researcher. I also thank Dr. Jen Noll and Dr. Cynthia Brown for their valuable feedback as I defended both my proposal and my dissertation.

I also thank the twenty-two students who participated in my study, as well as the handful of students who I interviewed in pilot studies over the years. Their generosity with their time and talents is greatly appreciated. Additionally, I thank fellow graduate student Steve Strand who worked alongside me on the project and who provided a number of helpful insights and ideas as I carried out my study.

There are two academic colleagues and dear friends to whom I feel most thankful and indebted. I thank Craig Swinyard, my academic older brother, who has on numerous occasions patiently read drafts, edited presentations, and engaged in productive 
conversations. Even more, at many times he kept me sane throughout the process, providing a listening ear or a word of encouragement when I needed it most. I also thank Dr. John Caughman, who has devoted countless hours to get me to this point in my career. My love for combinatorics began in his classroom, and his sheer enthusiasm for the subject (and for learning in general) has made an ineffable impression on me as a student, a teacher, and a researcher. It has been a joy to learn from him and a privilege to have his ideas mold and shape my own.

A number of other family members and friends from high school, college, grad school, and church have been a wonderful source of support over the last several years. I thank each of them for their continued love and encouragement.

Finally, and most importantly, I thank my parents. Their love has been unconditional, and they have provided steadfast support and encouragement whenever I have needed them. They have provided excellent examples of what it means to love learning and to value hard work. I am truly blessed to be their daughter, and I joyfully dedicate this dissertation to them. 


\section{Table of Contents}

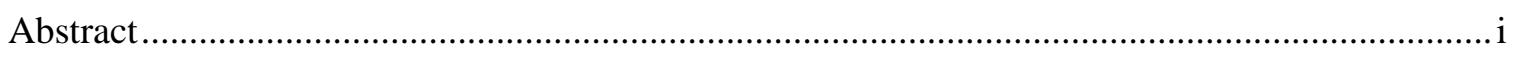

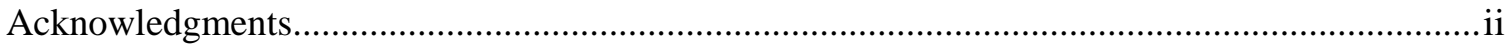

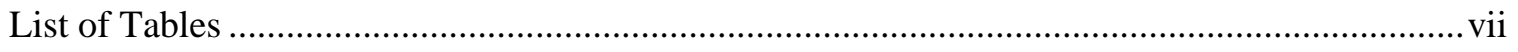

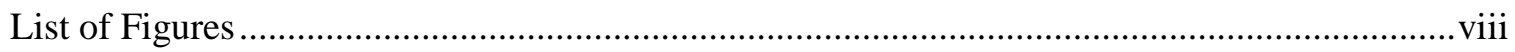

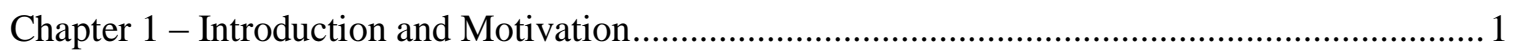

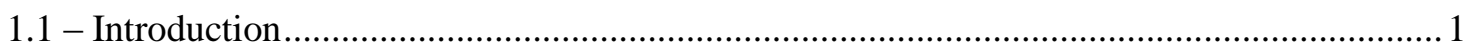

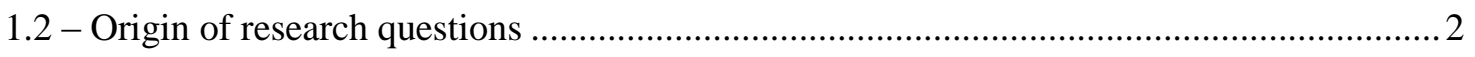

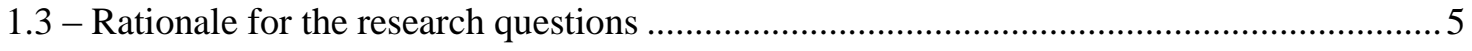

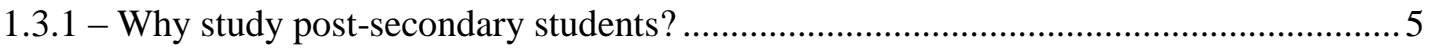

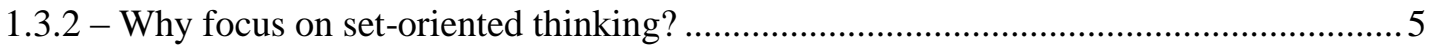

1.3.3 - Why focus on the particular error of over-counting? .............................................. 6

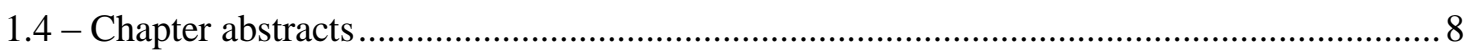

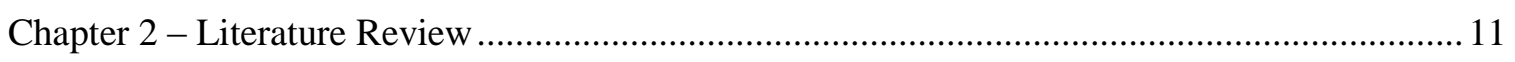

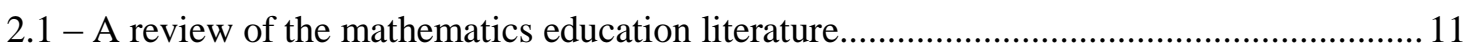

2.1.1 - A brief overview of existing literature on combinatorics in mathematics education. 11

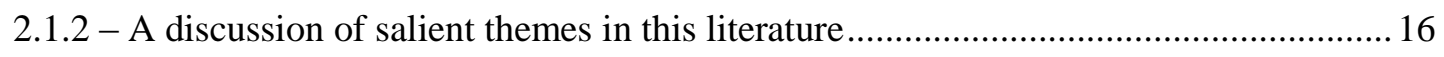

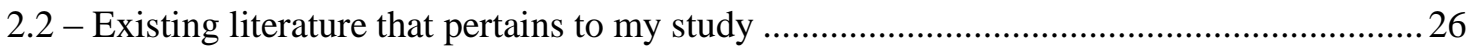

2.2.1 - A discussion of relevant mathematical notions as they relate to and inform set-

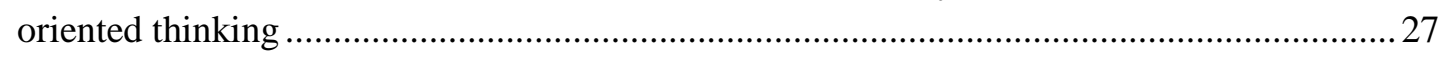

2.2.2 - A discussion of relevant mathematical notions related to errors of overcounting ..... 40

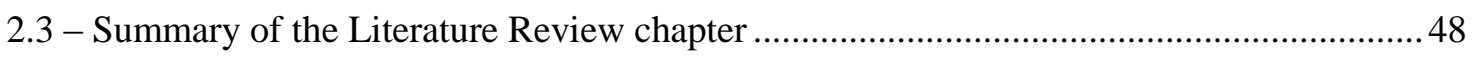

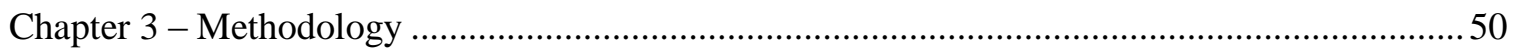

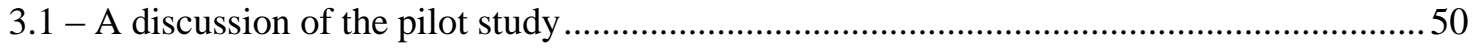

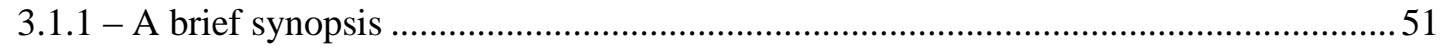

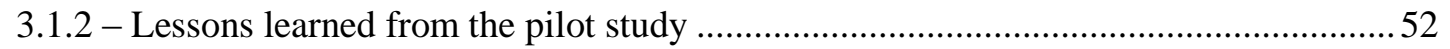

3.2 - Description of, and rationale for, the overall design of the study ...................................55

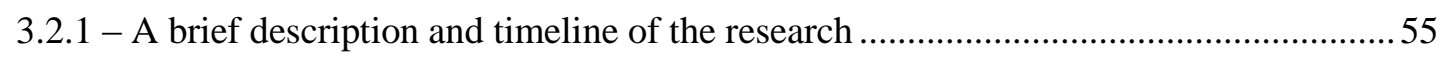

3.2.2 - Further description and rationale of the overall design of the study ..........................56

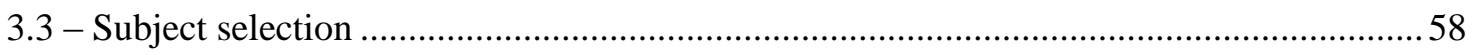




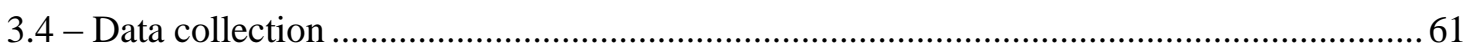

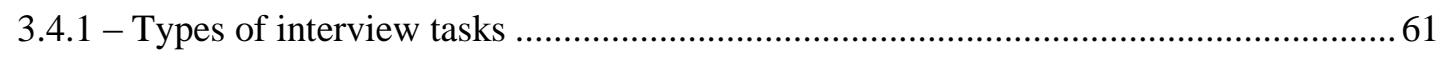

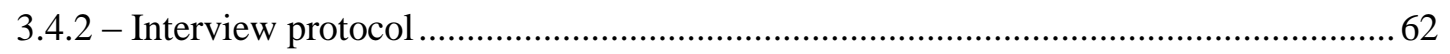

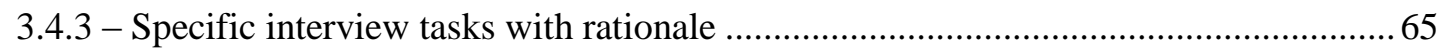

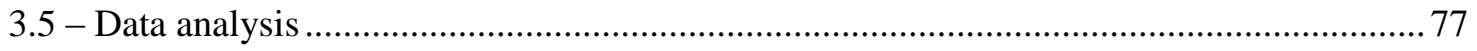

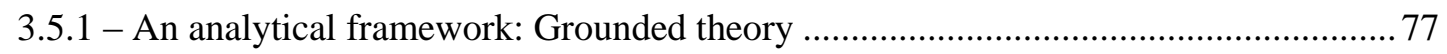

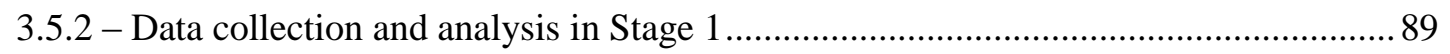

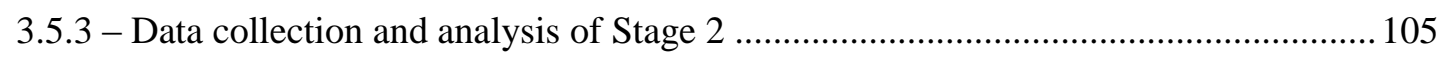

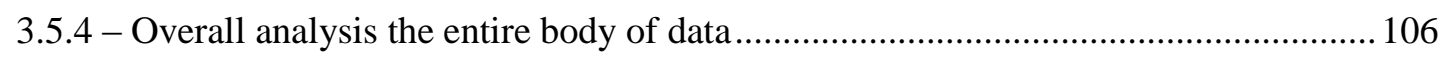

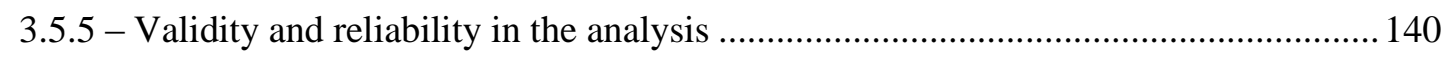

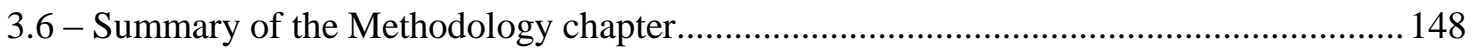

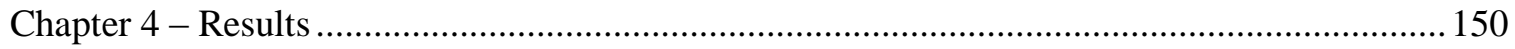

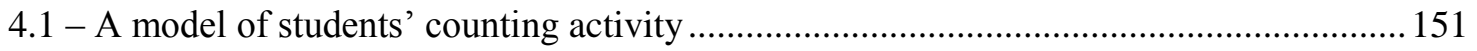

4.1.1 - Key relationships between components of the model........................................... 152

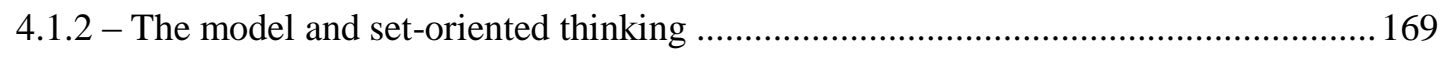

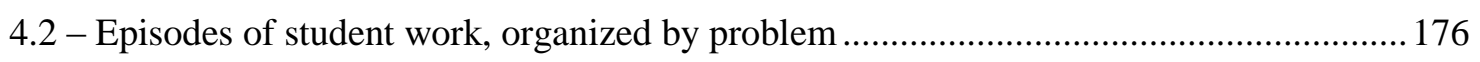

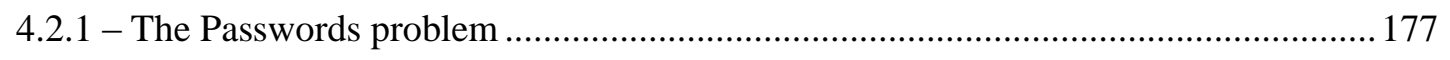

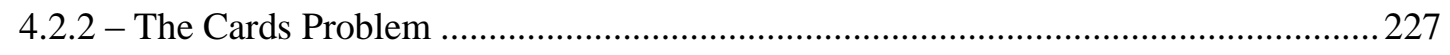

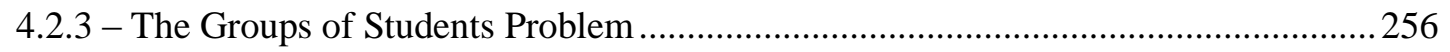

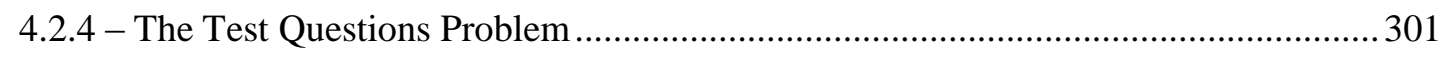

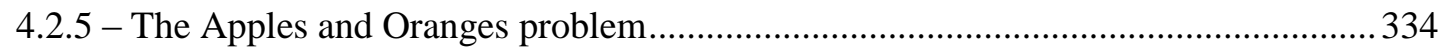

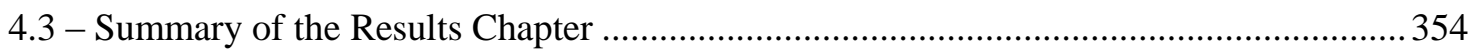

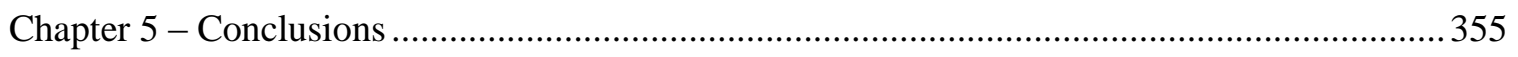

5.1 - A review of the model for the coordination of formulas, counting processes, and sets of

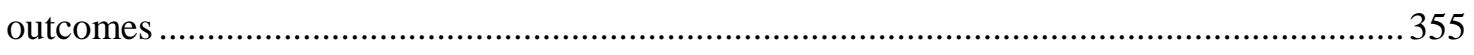

5.2 - Student work that did not indicate set-oriented thinking ............................................ 358

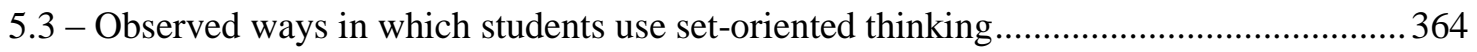

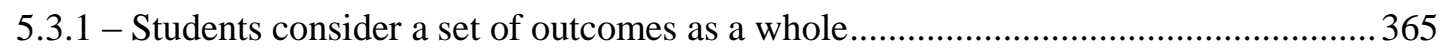

5.3.2 - Students appealed to some specific element of the set of outcomes ......................... 377

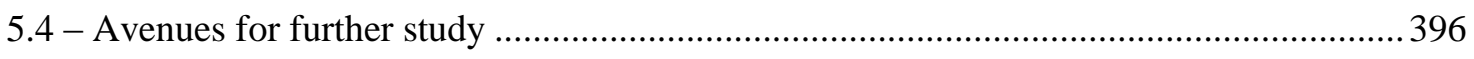

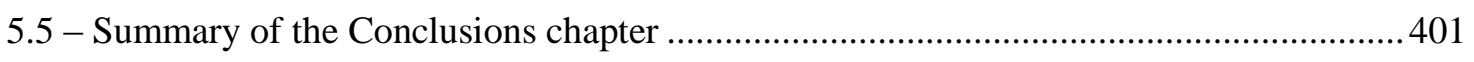




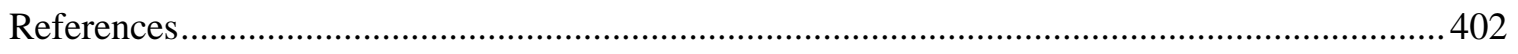

Appendix A - Counting survey used in student selection ..................................................... 413

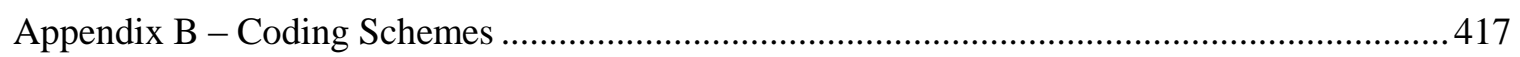

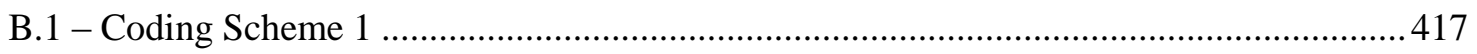

B.1.1 - Coding Scheme 1 Front Page .............................................................................. 417

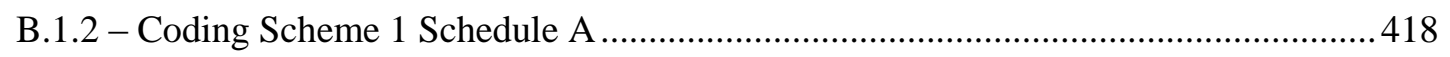

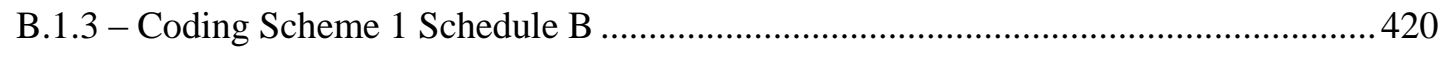

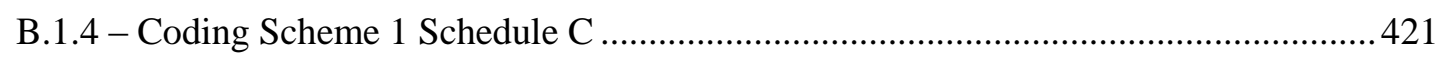

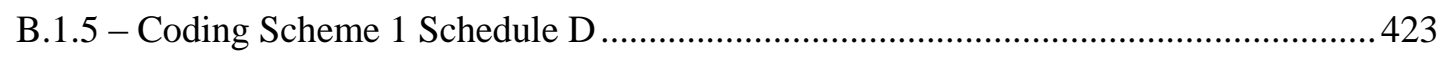

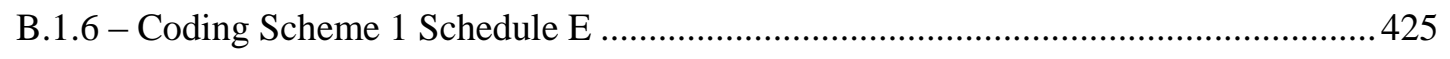

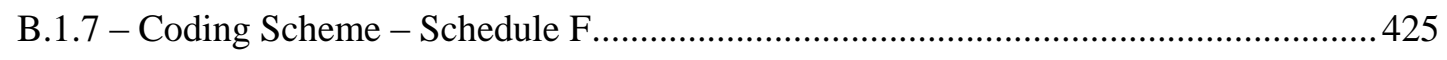

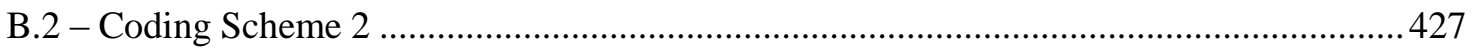

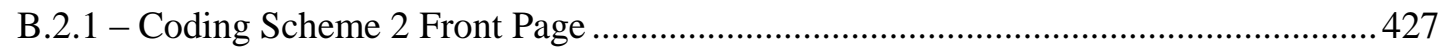

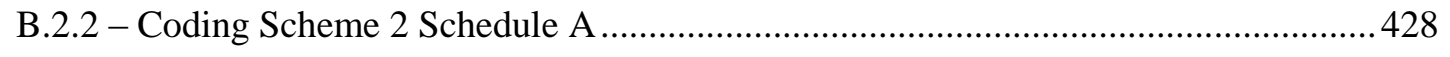

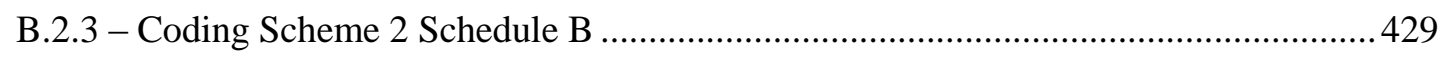

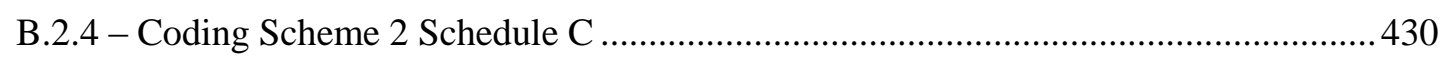

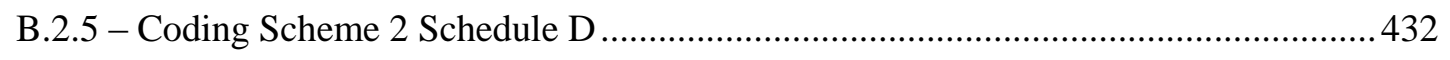

B.2.6 - Coding Scheme 2 Schedule E ........................................................................ 433

B.3 - Results of double-coding of Coding Scheme 1 ........................................................... 434

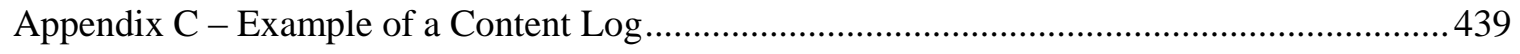




\section{List of Tables}

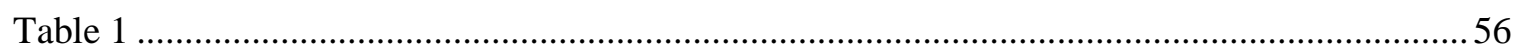

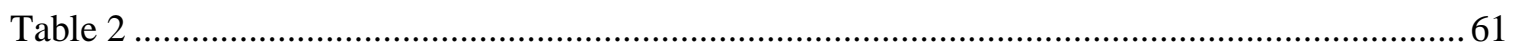

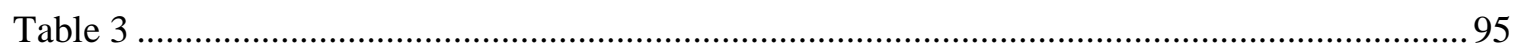

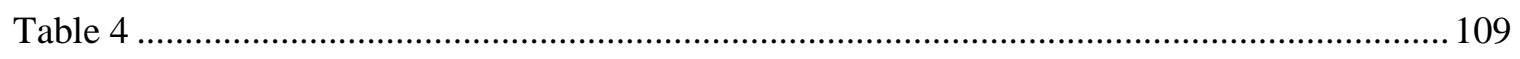

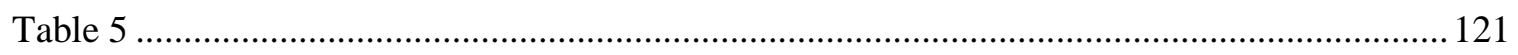




\section{List of Figures}

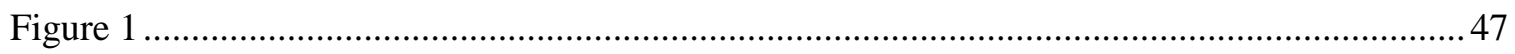

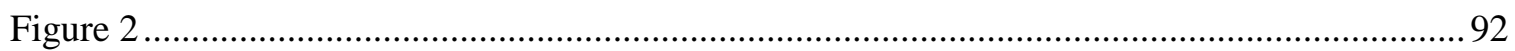

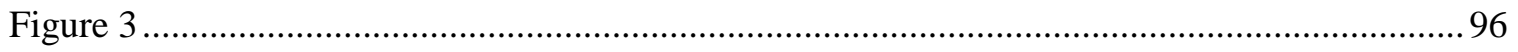

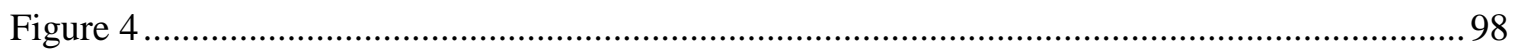

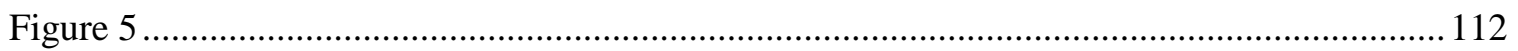

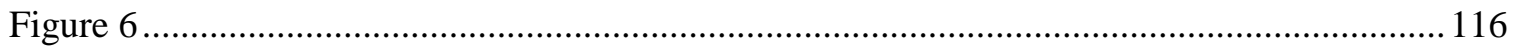

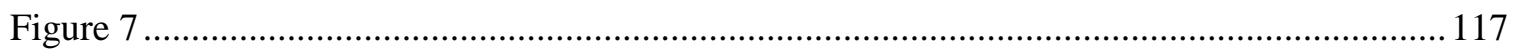

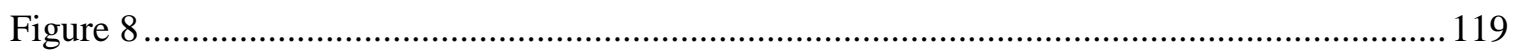

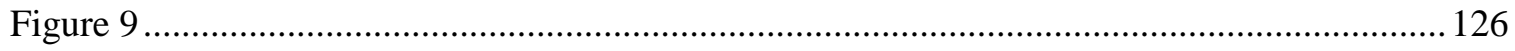

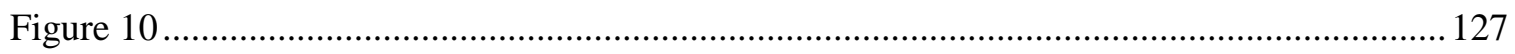

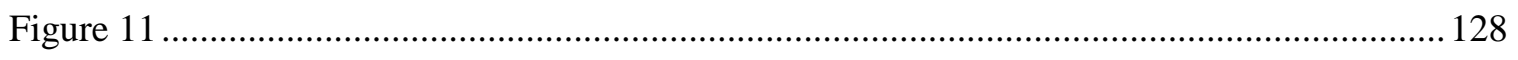

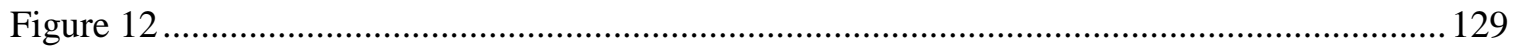

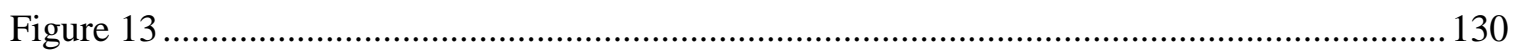

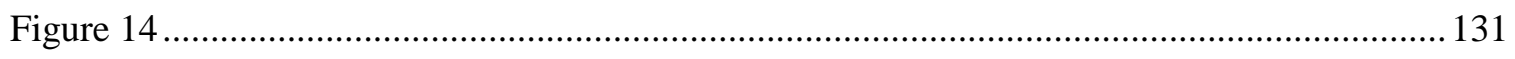

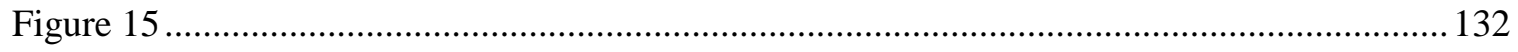

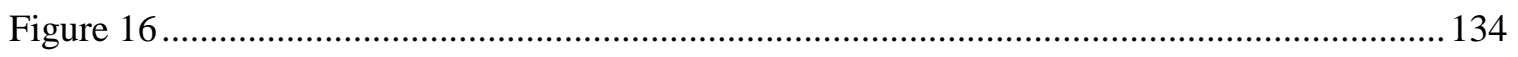

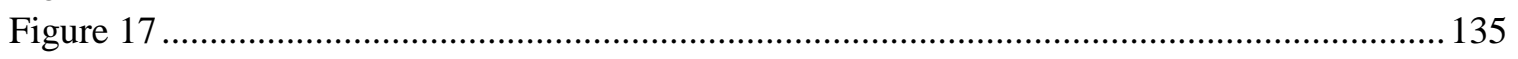

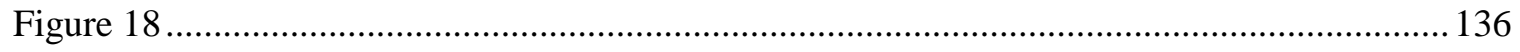

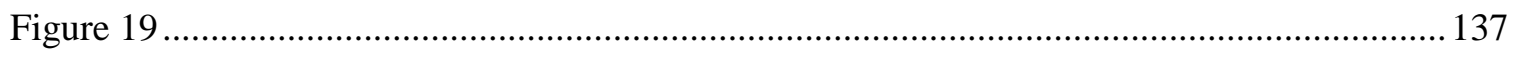

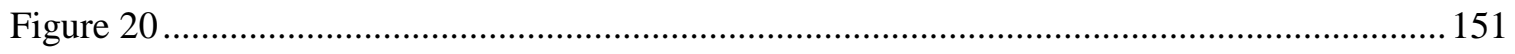

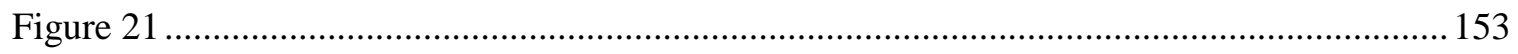

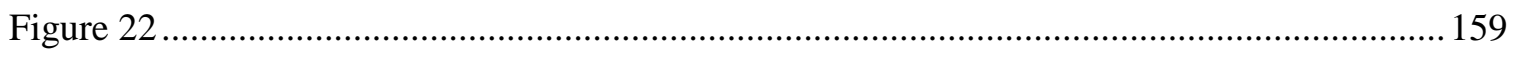

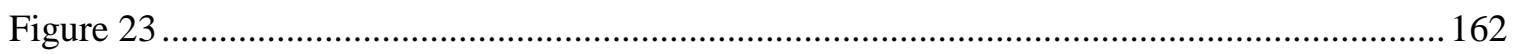

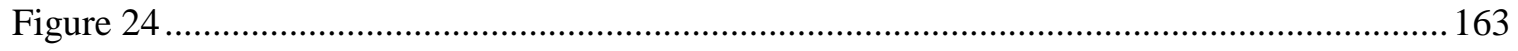

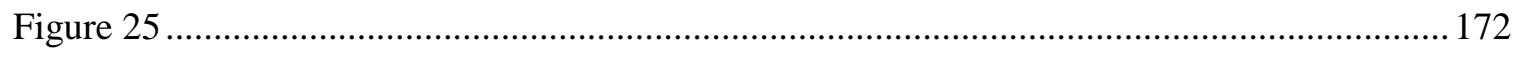

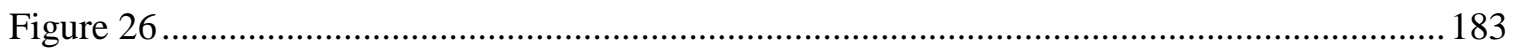

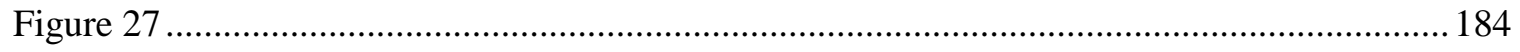

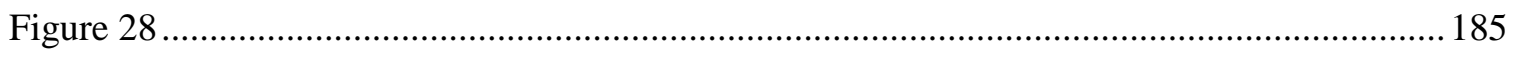

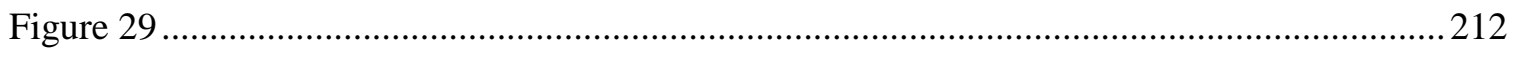

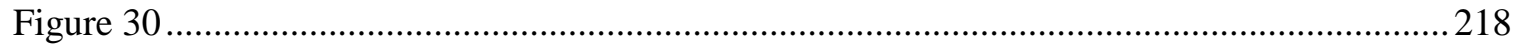

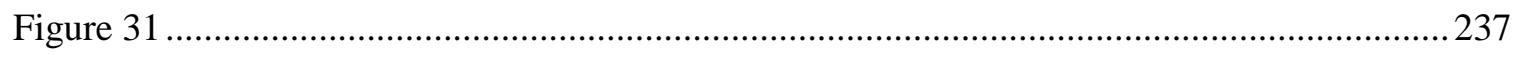

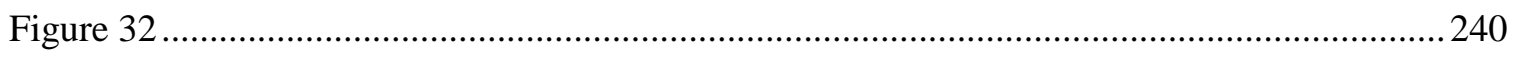

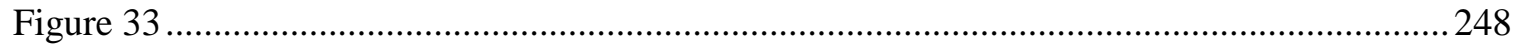

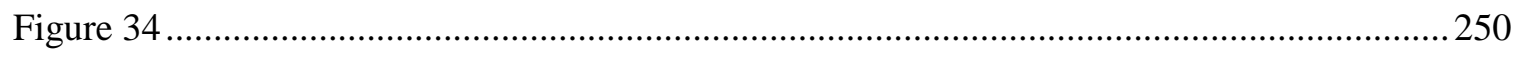

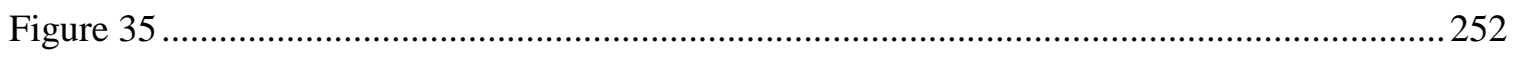

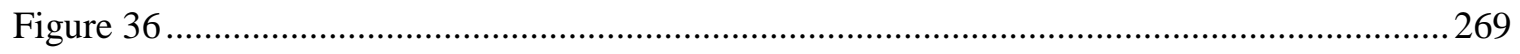

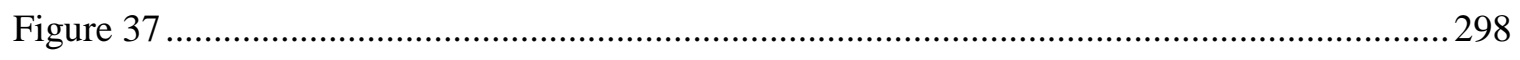

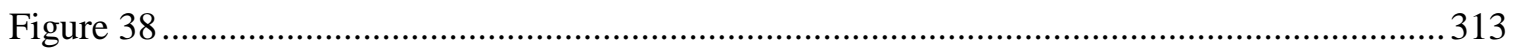




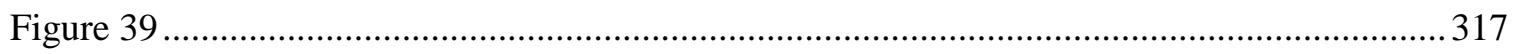

Figure 40

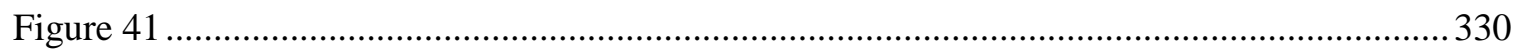

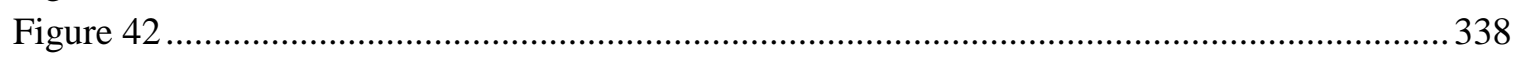

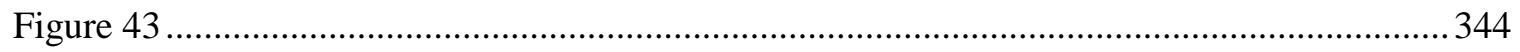

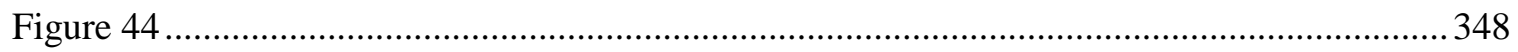

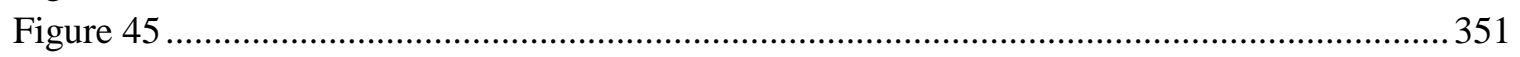

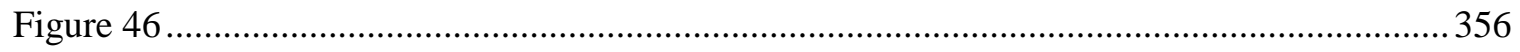

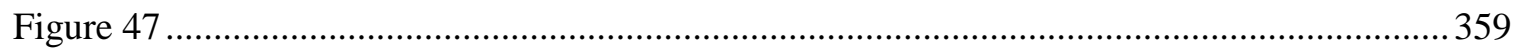

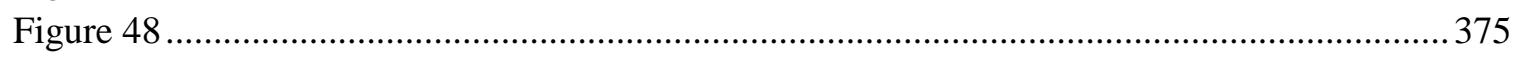

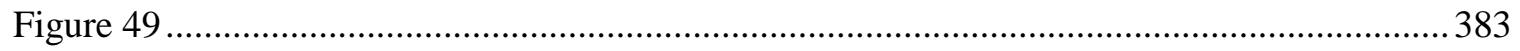

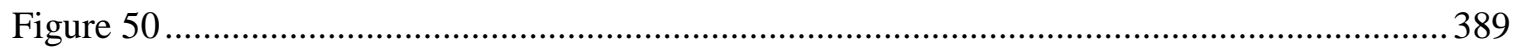

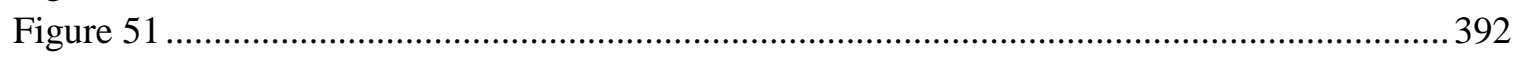


Lockwood Dissertation - 1

\section{Chapter 1 - Introduction and Motivation}

\section{1 - Introduction}

The activity of counting is among the most natural of intellectual processes. Indeed, it is one of the earliest mathematical activities that young children encounter (Lipton, 2006). As students advance mathematically, however, they tend to experience a great deal of difficulty as they come across increasingly complex counting problems; these difficulties are well-documented in the mathematics education research literature (e.g., Batanero, Godino \& Navarro-Pelayo, 1997; English, 2005; Kavousian, 2006). Also wellestablished in the literature is the relevance of combinatorics in the K-12 and undergraduate curricula (e.g., Batanero, Navarro-Pelayo, et al., 1997; English, 1991; Kapur, 1970; NCTM, 2000), particularly because of its applications in probability and computer science. Because combinatorics is becoming increasingly relevant in our computerized age, so, too, is the importance of knowledge and pedagogy related to combinatorics. Sriraram and English (2004) emphasize the mathematically robust aspect of combinatorial problems, maintaining that:

Combinatorial problems thus constitute a rich opportunity for mathematical exploration. In addition, combinatorics is an extremely useful branch of mathematics with numerous current applications in communications, authentication coding, coloring, and packing problems. Combinatorics has an intricate web of theoretical connections with many areas in mathematics (p. 189).

English (1993) confirms the value in studying the teaching and learning of combinatorics, noting that "the domain of combinatorics is a particularly fertile field for research in mathematics education" (p. 451). Attempts have been made to improve the 
implementation of combinatorial topics in the classroom (e.g., Kenney \& Hirsch, 1991; NCTM, 2000), but in spite of such efforts, students overwhelmingly struggle with understanding the concepts that underpin this topic. Batanero, Godino and NavarroPelayo (1997) note the need for an improvement in this area and make the following claim:

All these reasons justify the interest in improving the teaching of the topic [of combinatorics]. Nevertheless, combinatorics is a field that most pupils find very difficult. Two fundamental steps for making the learning of this subject easier are understanding the nature of pupils' mistakes when solving combinatorial problems and identifying the variables that might influence this difficulty (p. 182).

This call by Batanero, Navarro-Pelayo, et al. acknowledges the difficulties described above, and it also highlights a need for a deeper look at students' mistakes that will help researchers comprehend the nature of these mistakes. In addition to emphasizing student errors, there is a need to better understand ways of thinking that students bring to combinatorial tasks.

\section{2 - Origin of research questions}

The following personal historical development should shed light on the origin of the research questions. Because of the apparent paradox that, as stated above, something as simple as counting can actually be quite complex and subtle, I have long been interested in studying the teaching and learning of combinatorics. Over the last several years I have made the most of opportunities to take and teach combinatorics-related classes, all the while enjoying the percolation of potential research questions in my mind. As I began to get more serious about what research on combinatorics education might look like, I 
focused on the literature with the hopes of developing realistic research questions. During this review of the literature, it became clear to me that two fundamental counting principles (arguably the two fundamental counting principles - the addition principle and the multiplication principle) were conspicuously present in combinatorics texts, but conspicuously absent from the corpus of mathematics education work. This realization led me to focus, for a time, on studying how students might think about and understand these two principles. In order to do this, and in order to study what it might mean for students to understand these principles, I developed conceptual analyses of the addition and the multiplication principles. During this process, a particular feature of these principles (and of counting more generally) began to surface - the importance of setoriented thinking in one's counting activity. (I would go on to define set-oriented thinking as combinatorial thinking that involves attending to sets of outcomes in some way; this idea is defined more fully in the Results chapter). With this emphasis on sets in mind, I conducted a pilot study, focusing in particular on students' understandings of the addition and multiplication principles (and, secondarily, on ways in which set-oriented thinking arose for students as they solved counting problems). What emerged from this experience was a realization that an investigation of set-oriented thinking as it relates to counting problems may have some specific, potentially positive effects on our knowledge of what would support students in solving counting problems. I also saw that set-oriented thinking was a particular aspect of counting that I could investigate which could relate meaningfully to both the addition and multiplication principles (and many others besides). 
I reviewed the pilot study data and also reflected on my own experiences as someone who had both taught and learned counting over a number of years. As I considered what set-oriented thinking might entail, as well as the ways in which such a perspective could be leveraged in counting problems, specific instances of such thinking repeatedly came to mind. Each of these instances can be characterized as relating to a notoriously prevalent source of student confusion, namely, errors of over-counting. Upon further investigation, I found that the widespread occurrence of errors of over-counting was indeed substantiated, both by the combinatorics education literature (e.g., Hadar \& Hadass, 1981; Mellinger 2004) and in several combinatorial textbooks (e.g., Bona, 2007; Tucker, 2002). In addition, further evidence of the occurrence of these particular errors was borne out in both of my pilot studies (Lockwood, 2009; Lockwood, 2010). Therefore, the focus on this particular type of error stemmed from personal experience of the author (further underscored by personal communication with a combinatorialist), from research literature on mathematics education, from combinatorial texts, and from pilot study data.

Thus, the overall purpose of this study is to examine ways in which set-oriented thinking arises as post-secondary students solve counting problems that are susceptible to errors of over-counting. This study seeks to address the following research questions (a broad question and a more specific one):

To what extent is set-oriented thinking relevant as post-secondary students solve counting problems? Specifically, in what ways do students use set-oriented thinking as they solve and evaluate counting problems that are susceptible to errors of over-counting? 
Lockwood Dissertation - 5

\section{3 - Rationale for the research questions}

\subsection{1 - Why study post-secondary students?}

Studying post-secondary students allows me to investigate counting problems that enjoy a certain level of sophistication. It is likely that some high school students could also handle complex counting problems, but work with undergraduates and graduate students allows for the investigation of mathematical subtleties that might not arise in basic counting problems. This flexibility is important, as it allows a more precise identification of what might involve subtle aspects of set-oriented thinking. Additionally, I am generally most interested in the post-secondary population, both as a researcher and a teacher. I more clearly outline specific subject selection decisions in Section 3.3.

\subsection{2 - Why focus on set-oriented thinking?}

I take set-oriented to mean that thinking which involves attending to a set of outcomes (the cardinality of which often determines an answer to a counting problem). Indeed, this thinking indicates that, to the counter, the task of counting amounts to counting the number of objects in a set. Intrinsic in this is a conceptualization of the things to be counted as a set of outcomes - a set which people can manipulate as they would any other set. This means that they can conduct membership tests to determine elements in the set, they can consider subsets or partitions of the set, they may consider the union or intersection of that set with other sets, and so on. According to this way of thinking, then, counting techniques or procedures can be viewed as ways to organize this set of objects, producing structure that can render the enumeration of that set more 
manageable. When I talk about conceiving of a counting problem in a set-oriented way, it is not the case that students need to be able to picture or produce an exhaustive list of every object to be counted; this may often not be possible. Rather, set-oriented thinking is characterized by the capacity conceive of the act of counting as counting some set of outcomes and to draw upon the set of outcomes during one's counting activity.

While the construct of set-oriented thinking is not well-documented among the research literature, there are some studies that suggest that such thinking is at least a plausible way of thinking about combinatorial tasks (e.g., English, 1993; Hadar \& Hadass, 1981). Even more, a number of authors of combinatorics and discrete mathematics texts (e.g., Brualdi, 2004; Rosen, 2007; Tucker, 2002) acknowledge that ideas in counting can be formulated in a set-oriented way. In spite of this, no study has yet explicitly addressed the significance of this way of thinking, nor has a study investigated the ways in which set-oriented thinking may affect students' solving of counting problems. In addition to this, my own experience as a teacher and student of counting (and this has also been borne out by pilot studies) has led me to believe that setoriented thinking is potentially quite significant in students' success on counting problems.

\subsection{3 - Why focus on the particular error of over-counting?}

I do not emphasize the error of over-counting because I want to focus on a particular difficulty or misconception with which students struggle. Rather, this error is chosen because it provides a narrow (and therefore more manageable) context in which to study 
set-oriented thinking. Therefore, I want to be clear that the error itself is not the focus of study; rather, the study is focused on set-oriented thinking in the context of errors of over-counting. Nonetheless, the context of errors of overcounting is not an entirely arbitrary narrowing. There was some indication in the pilot study that there were perhaps substantial ways in which set-oriented thinking might arise in this context, so it seemed a fertile ground for a first study. I explain now why this error provides a desirable mathematical context for the examination of the research question.

The error of over-counting is generally warned against in combinatorial texts, as it is one of the ways that a counting problem can be incorrect - one may arrive at an incorrect answer to the question by arriving at an answer that is too small (thus under-counting) or too large (thus over-counting). However, there are particular ways in which overcounting occurs that are difficult to detect in a seemingly correct counting procedure. That is, it is possible for a counting procedure to generate too many solutions, but the error may be virtually undetectable to the untrained eye, and the procedure may seem logically solid. As mentioned above, in my own experience, errors of over-counting often seem to be detectable and correctable by set-oriented thinking. In particular, there are several problem types in which it seems to be the case that set-oriented thinking offers a valuable perspective that can enable students to detect and fix errors of over-counting. It is exactly this kind of relationship that I sought to explore through this study. That is, I investigated whether there are specific ways in which set-oriented thinking affects students' solutions of counting problems. 
Lockwood Dissertation - 8

\section{4 - Chapter abstracts}

Beyond this introductory chapter, this document is comprised of four more chapters, each devoted to a particular aspect of the study. I now provide abstracts of what will be covered in each of the respective chapters.

Chapter 2 is entitled Literature Review. In this chapter I both frame my study in the existing body of literature, and I elaborate the theoretical perspectives that frame my research study. The chapter consists of three major sections. In Section 2.1, I provide a brief, comprehensive review of the current mathematics education literature on combinatorics, including a detailed discussion of relevant themes in the combinatorics education literature. This section should give the reader an overall sense of what has been done previously and should reflect the need for more studies that investigate students' thinking about counting. In Section 2.2, I address how my study is situated within the existing mathematics education literature. To do this, I discuss the treatment of setoriented thinking in the literature (explicating some of the mathematics education literature about the relationship between processes and sets that is related to my study), and I provide my initial views of set and process-oriented thinking. I also further explore errors of over-counting and provide examples to explicate the relationship between setoriented thinking and such errors. I summarize the chapter in Section 2.3.

Chapter 3 is the Methodology chapter, and in it I describe the specifics of how the study was conducted, detailing each of its three overall phases: student recruitment, data collection, and data analysis. In Section 3.1, I briefly describe the pilot study that informed the design of the proposed study. In Section 3.2, I provide a description of the 
overall design of the study, and I offer rationale for particular decisions. In Section 3.3 I provide details for subject selection, and in Section 3.4 I outline specifics of the data collection process. In Section 3.5 I detail the data analysis that I conducted, providing examples of instruments in order to explain and justify my analytical decisions. This includes an in-depth discussion of the methodological framework of grounded theory (as put forth by Auerbach and Silverstein (2003) and including aspects of Strauss and Corbin (1998)). In Section 3.6 I provide a summary of the chapter.

Chapter 4 is the Results chapter, and there are two major parts to the chapter. In Section 4.1 I present a model for the coordination of formulas, counting processes, and sets of outcomes. This model is a conceptual analysis that emerged as a result of my study, and it was instrumental in formulating my ideas regarding set-oriented thinking. In this section I introduce and describe relevant elements of the model. In Section 4.2 I give the reader a sense of what occurred in the interviews. I organized the section according to problem. Specifically, I give detailed treatments of student work on each of the five interview tasks. This involves in-depth descriptions of the students' mathematical activity as they engaged with the Passwords problem (Section 4.2.1), the Cards problem (Section 4.2.2), the Groups of Students problem (Section 4.2.3), the Test Questions problem (Section 4.2.4), and the Apples and Oranges problem (Section 4.2.5). In Section 4.3 I summarize the Results chapter.

Chapter 5 is the Conclusions chapter. This chapter contains the major findings from my dissertation; it is here that I draw the conclusions from my analysis of the data and address the research questions. In Section 5.1 I remind the reader of the model described 
in the Results chapter. In Section 5.2 I give evidence of student work that suggested they did not, at times, use set-oriented thinking. In Section 5.3 I put forth a potential framework that outlines some of the observed ways in which students did use set-oriented thinking in their counting activity. Specifically, I provide examples in which students referred to the entire set of outcomes and I also note instances in which students appealed to particular elements of the set of outcomes. This framework is my initial attempt at categorizing observed instances of set-oriented thinking. In Section 5.4 I propose specific avenues for further study that could come out of my work, and I finish the Conclusions chapter with some final remarks about my findings in Section 5.5. 


\section{Chapter 2 - Literature Review}

In this chapter, I first provide a full review of the mathematics education literature on combinatorics, including a discussion of relevant themes and ideas. Then, I address a handful of particular studies in the literature that pertain to my study. This includes a brief exploration of the mathematics in which my study is rooted, which involves both the development of my ideas regarding set-oriented thinking, and details about errors of overcounting that have played into my development of the study. I conclude with a summary of the chapter.

$2.1-$ A review of the mathematics education literature

2.2 - Existing literature that pertains to my study

2.3 - Summary of the Literature Review chapter

\section{1 - A review of the mathematics education literature}

\subsection{1 - A brief overview of existing literature on combinatorics in mathematics education}

There is a relatively small body of literature available on the teaching and learning of combinatorics. In an effort to give the reader a sense of what is available and what has been done, I briefly summarize this body of work below. The literature could be categorized in a variety of ways, and I offer a more detailed discussion of relevant themes in the next section, but in this section the goal is simply to provide a comprehensive picture of available research on combinatorics education, without expounding in detail upon particular studies. This section is therefore intentionally compact and concise. 
2.1.1.1 - K-12 studies. The majority of work on the teaching and learning of combinatorics has been performed at the K-12 level. Some of the most well-known work was conducted with young children by Piaget in his studies of the concept of chance (Piaget \& Inhelder, 1975), in which he interviewed young students in an effort to determine ages at which they could learn combinatorial concepts such as combination and permutation. In several of her papers (e.g., English, 1991; English, 1993), English builds upon Piaget's work by examining young children's combinatorial strategies, focusing especially on their activity on elementary counting problems involving the multiplication principle. In Tillema's (2007) dissertation he offers significant analysis of middle school students' work on multiplicative combinatorial problems. In addition to this, a major strain of the longitudinal study conducted by Maher (2002) saw students solving combinatorial problems, and thus a number of papers have emerged from this study in which combinatorics plays a major part (even if combinatorics is not itself the major focus of study). Examples of such studies include Maher \& Martino, (1996), which focuses on students' proof and justification on a combinatorial task, Powell \& Maher (2003), which examines heuristics of students' building of isomorphisms as they solved a combinatorial problem, and Warner (2008), which investigates Pirie \& Kieran's (1994) model for the growth of mathematical understanding of a combinatorial concept. Maher \& Speiser (1997) used a combinatorial task involving block towers to investigate higherlevel mathematical ideas with students. Two other studies focus on high school students as they study combinatorics. In particular, Fischbein \& Gazit (1988) examine the effect of instruction on high school students' solutions of combination and permutation 
problems. In this study, they highlight the difference between types of combinatorial operations, as well as the various natures of elements involved, and they suggest that these factors have a bearing on students' abilities to solve counting problems. In a study with a similar emphasis, Batanero, Navarro-Pelayo, \& Godino (1997) examine the effects of problems' implicit combinatorial models.

2.1.1.2 - Undergraduate studies. There are also studies in combinatorics education that focus on undergraduate students. Hadar and Hadass (1981) suggest a number of pitfalls that undergraduate students may face when studying combinatorics. Mellinger (2004) articulates errors of order among undergraduates and suggests ideas for ways in which to address such issues in the classroom. Additionally, Eizenberg and Zaslavsky have conducted studies on cooperative problem solving (2003) and students' verification strategies (2004) specifically within the realm of combinatorics. Godino, Batanero and Roa (2005) focus on undergraduates as they look to use combinatorics as an example through which to introduce a particular framework (an onto-semiotic model of learning). Kavousian (2008) performed studies in two veins - one examined students' work with a novel combinatorial definition as it pertained to their ability to solve combinatorics problems, and the other discussed student-generated examples as a means by which to take advantage of a teachable moment in which students incorrectly solved a counting problem. In another study, Engelke and CadwalladerOlsker (2010) examined undergraduate and graduate students' work with problems involving combinatorial proof. They indicated that students struggled with such problems and put forth a categorization of errors. Finally, Lockwood (2011) used Lobato's (2003) notion of actor-oriented 
transfer in order to analyze the connections that undergraduate students made between counting problems.

A handful of works provide surveys of the combinatorics literature, including book chapters by English (2005) and Batanero, Godino \& Navarro-Pelayo (1997). Srirarim and English (2004) also offer insight into the relationship between research and practice by synthesizing the literature and applying it to instruction. In addition, there are papers that adopt a cross-sectional approach in their studies. For example, Kapur (1970) conducted a study on student learning across a variety of ages, as did Fischbein and Grossman (1997) in their discussion of schemata and intuitions in a combinatorial context.

2.1.1.3 - Discrete mathematics. In 1989, NCTM highlighted the need for the incorporation of discrete mathematics into the K-12 curriculum (NCTM, 1989), which resulted in an NCTM publication entitled Discrete Mathematics across the Curriculum (Kenny \& Hirsch, 1991). This book features chapters (e.g., Althoen, Brown, \& Bumcrot, 1991; DeGuire, 1991; Dossey, 1991; Evered \& Schroeder, 1991; Gardiner, 1991; Holliday, 1991; Miller, 1991; Schielack, 1991; Spangler, 1991) on the teaching of discrete mathematics, and it includes a number of chapters on combinatorial topics. These chapters do not provide results from research on the teaching and learning of combinatorics; instead, they offer suggestions for the incorporation of these ideas into the classroom. Additionally, in 2004, Zentralblatt für Didaktik der Mathematik (ZDM, now the International Journal on Mathematics Education) published a special issue on discrete mathematics, and several articles (e.g., Debellis \& Rosenstein, 2004; Ebert, Ebert, \& Klin, 2004; Schuster, 2004) emerged from that volume. Like the NCTM publication, 
these articles emphasize the practical implication of discrete mathematics instruction in the classroom, and they do not speak specifically to research on the teaching and learning of combinatorics. Similarly, Abromovich and Pieper (1996) offer a non-empirically based paper on fostering recursive thinking in combinatorics through the use of manipulatives and computing technology. In another non-empirically based article on discrete mathematics, Grenier and Payan (1999) suggest discrete mathematics as a tool for learning proof and modeling.

2.1.1.4 - Probability and Statistics. Combinatorial ideas also arise in research on the teaching and learning of probability. Some counting ideas (particularly independence) show up in a number of works on probability education (e.g., Fischbein, Nello, \& Marino, 1991; Fischbein \& Schnarch, 1997; Hietele, 1975; Jones, Langrall, Thornton, \& Mogill, 1999; Konold, Pollatsek, Well, Lohmeier, \& Lipson, 1993; Shaughnessy, 1977; Rubel, 2007). Most often, combinatorial activity (specifically enumeration) arises when students count sample spaces in problems involving probability. In particular, Thompson \& Saldanha (2002) relate students' sampling to their conceptualization of a set of outcomes. English (2005) suggests that studies on probability "indicate that children have difficulty with basic probability ideas because they are not able or not willing to construct combinatorial type outcomes" (p. 133). Thus it seems that counting issues can arise as students consider outcomes and sample spaces in their work with probability.

2.1.1.5 - Other topics. Finally, on occasion, combinatorial topics arise in the mathematics education literature, but combinatorics itself is not the focus of the study. That is, a combinatorial setting or idea may be used as a means or a context by which to study 
some other area. Some of these topics include, for example, mathematical thinking (Maher, 2002), the realization of problem solving techniques (Mamona-Downs and Downs, 2004), school versus non-school mathematics (Schliemann \& Acioly, 1989), and information processing (Scardamalia, 1977).

In summary, although the entire body of work on combinatorics is relatively small, there are a number of topics and ideas that have been addressed by researchers. While there is a base of research from which to set, there forth is still much room for growth in this field. The reader may notice that while there are a number of articles and chapters related to mathematical teaching and practice (such as the NCTM publication Discrete Mathematics Across the Curriculum (Kenney \& Hirsch, 1991) and the 2004 ZDM publication), there is limited work available on ways of thinking and learning that pertain to combinatorics.

\subsection{2 - A discussion of salient themes in this literature}

In my study of the literature described above, there are several themes that have emerged. In this section, I synthesize and elaborate some of these themes. While these themes do not necessarily pertain precisely to the direction that my dissertation research will take, they should further orient the reader to prominent aspects of the literature. This background should help to situate my research study among the existing literature. These themes are: Difficulties with counting, Underlying structures, Representations, and Problem solving strategies. 
2.1.2.1 - Difficulties with counting. A primary theme in the mathematics education literature is that combinatorics (and the solving of combinatorics problems) does, in fact, seem to be challenging for students. Examples of this can be seen in Kavousian (2008), who studied undergraduate students who had been exposed to combinatorial ideas in previous classes. While working through a selection with repetition problem, one student said, "I used to know these things...I just can't seem to picture it anymore...I know this but somehow it's just not coming" (p. 90). This indicates a struggle with the recollection of combinatorial ideas and suggests that the particular model was perhaps initially not learned sufficiently. In addition to this, Godino et al. (2005) note that in Roa's (2000) study, 118 undergraduate mathematics majors were given a questionnaire with thirteen "simple" (Godino et al., 2005, p. 4) combinatorial problems. They note that these students "generally found it difficult to solve the problems (each student only solved an average number of 6 problems correctly)" (p. 4). Eizenberg and Zaslavsky (2004) similarly state that their findings "support the assertion that combinatorics is a complex topic - only 43 of the 108 initial solutions were correct” (p. 31). Additionally, Hadar and Hadass (1981) state that, "combinatorics is a field which most of the students find very complicated” (p. 436). Other researchers (e.g., Batanero, Navarro-Pelayo, et al., 1997;

\footnotetext{
${ }^{1}$ Godino et al. (2005) do not explain what they mean by simple. It appears to mean that the problems were straightforward problems about a particular combinatorial operation (such as combination, permutation, etc.), free of any particularly tricky or deceptive elements. An example of such a problem is found on page 9: "Problem 3: A boy has four different colored cars (black, orange, white and green) and he decides to give out the cars to his friends Fernando, Luis and Teresa. In how many different ways can he distribute the cars? For example, he could give all the cars to Luis."
} 
Fischbein \& Gazit, 1988) also agree with the claim that combinatorial problems are nontrivial for students.

Several authors of combinatorial texts echo the above claim that counting is, in fact, difficult. Tucker (2002), for example, says that in his counting chapter "we discuss counting problems for which no specific theory exists" (p. 169) and calls it "the most challenging and most valuable chapter in this book" (p. 169). In fact, the first section of Martin's (2001) book on counting is entitled "Counting is Hard." He points out that "there are few formulas and each problem seems to be different" (p. 1). And in his forward to Bona's (2007) book, combinatorialist Richard Stanley marvels at the fact that a basic mathematical activity like counting can lead to rich mathematics and ingenuity. In addition to this, there are places in which the textbook authors put forth strategies for solving combinatorial problems; these underscore that counting is far from an algorithmic, predictable discipline. Tucker suggests that logical reasoning, clever insights, and mathematical modeling are necessary skills for success in combinatorics. Martin good-naturedly warns that "you will have to think" (p. 1) in order to solve problems, especially in light of the lack of algorithms in the subject. Thus, there appear to be very real challenges for students to be successful at counting, and this may serve as motivation to find ways to ameliorate this condition.

In spite of such difficulties, there are also instances of success for students. For example, although English (1991) acknowledges difficulties that her young students faced, she provides evidence that young children are able to perform combinatorial tasks at younger ages than those initially put forth by Piaget and Inhelder (1975). Additionally, 
Maher and her colleagues (e.g., Maher \& Martino, 1996; Powell \& Maher, 2003) offer instances of powerful combinatorial reasoning by students at a variety of levels. Thus, we see in the literature that although combinatorics is undoubtedly difficult for students, there is also evidence that combinatorics is a potentially accessible topic in which students may thrive. The potential success that students may experience serves as further motivation to help students engage in this challenging but potentially rewarding topic.

2.1.2.2 - Underlying structures. English (2005) is one of several researches who indicate the need to focus on problem structures. In her work with young students, she suggests that if children are indeed going to make important relationship links, "they need to construct understandings that comprise the structural relations between ideas, not the superficial surface details" (p. 135). She goes on to argue that children should focus on underlying problem structures, and not just on the surface, context-specific features of a problem. However, she also notes that "a common finding in many of the studies on combinatorics is that students have difficulty in identifying related problem structures" (p. 135). Thus, she suggests that their ability to transfer learning to other problems in different contexts is underdeveloped, and it is something with which they struggle. Elsewhere, in their work connecting research to practice, Sriraman and English (2004) note that a common implication of the research is that "choosing problems that vary contextually but are essentially similar in their mathematical structure is pedagogically important" (p. 186). Therefore, we begin to see the importance of the underlying structure of problems in order to foster the effective development of relationships between combinatorial (and other relevant mathematical) ideas and concepts. 
To unpack this theme, I examine several specific structures that some researchers have identified as being of particular importance. Piaget and Inhelder (1975) suggest fundamental differences between the mental processes that combinations and permutations each respectively require, conjecturing that permutations occur at a more formal thought level than combinations. In particular, according to Batanero, NavarroPelayo, et al. (1997), Piaget conjectured that while "combinations involve the coordination of seriation and correspondence, permutations imply an arrangement according to a mobile and reversible system of reference; therefore they are operations on operations, characteristics of the formal thought level" (p. 182). Fischbein and Gazit (1988), building upon this idea (and essentially finding counterexamples to Piaget's conjecture), put forth combinatorial operation as a potentially relevant factor in successful problem solving; combinatorial operations include operations such as permutations and combinations. In particular, Fischbein and Gazit found that, prior to instruction, the order of difficulty of combinatorial operations was: permutations, arrangements with repetition, arrangements without repetition, and combinations (this agreed with Piaget's theory). However, they found that, after instruction, combinations were found to be more difficult for students than permutations. Thus, an underlying structure of a combinatorics problem might be characterized by the type of combinatorial operation that the problem requires. Fischbein and Gazit also examined the nature of elements that were used in problems, in order to determine whether the element type in a problem had an impact on student success. They found that students could work more easily with problems involving digits rather than those that dealt with committees or 
colored flags, which they explain by noting that students are more familiar with operating with numbers than with other objects.

In another attempt to get at underlying structure, Dubois (1984) suggests that combinatorial configurations could be classified into three models: selections, distributions, and partitions. Selections evoke a notion of sampling, distributions bring to mind mapping, and partitions suggest the division of a set into subsets. Dubois also proposes that there are important distinctions that can be made even within the models ${ }^{2}$. In the selection model, such distinctions relate to whether replacement is allowed and whether the samples are to be ordered or not. In the distribution model, relevant features are whether the objects to be distributed are identical or not, whether the containers are identical or not, and whether we must order the objects placed into the containers. Therefore, we see another proposed way in which researchers describe the underlying structure of combinatorics problems - namely, three models of classification. Building off of Fischbein and Gazit (1988), and the work of Dubois (1984), Batanero, NavarroPelayo, et al. (1997) designed a study that investigated the effects of both an implicit combinatorial model and particular combinatorial operations. They created a questionnaire that included problems of model type (distribution, selection, and partition), and within each of these models they included problems with varying

\footnotetext{
${ }^{2}$ For example, in the distribution model he suggested the following six basic types: ordered distributions of different objects in different containers, ordered distributions of different objects in identical containers, non-ordered distribution of different objects in different containers, non-ordered distributions of different objects in identical containers, distributions of identical objects in different containers, distributions of identical objects in identical containers.
} 
combinatorial operations (combinations, permutations with repetition, arrangement with repetition, permutations, and arrangements). The results from this study include a list of common misconceptions they found, as well as four factors that seemed to have an impact on their students' work. These factors include the complexity of the verbal statement of the problem, the combinatorial model, the specificity of permutation problems, and similarities and discrimination between combinations and permutation with repetitions. Finally, in an attempt to investigate students' understandings of similar situations and underlying structures, Lockwood (2011) used Lobato's (2003) framework of actor-oriented transfer. Actor-oriented transfer is an alternative to the traditional transfer perspective; the framework allows for researchers to investigate those connections that students (actors) generate between situations and not rely on transfer tasks that rely on an expert's notion of similarity. Lockwood's work suggests that while the underlying structure of a particular problem may be important, students may consider alternative kinds of underlying structures than teachers or researchers might expect.

2.1.2.3 - Representations. Although many of the results of Maher's longitudinal study (e.g., Glass \& Maher, 2004) focus on non-combinatorial ideas such as proof and justification, many of her studies still suggest trends that are relevant to combinatorics education. In Maher's studies, there is an indication that a prototypical problem was significant for students. That is, after prototypical problems were introduced, the students often later referred back to that prototype in subsequent work, and most often such a reference proved useful in the ensuing activity (this may also be related to the underlying structure theme described above). For example, their students worked on particular 
combinatorics problems that were called the Tower Problem and the Pizza Problem. After these specific problems were introduced, they were brought up again, by name, even years later. Indeed, when studying an additive relationship between terms in Pascal's Triangle in eleventh grade, one student referred back to work he had done with pizza toppings three years earlier (Maher, 2002, p. 12). Other examples of this can be seen in Powell and Maher (2007). In addition, other studies (e.g., Maher \& Martino, 1996) point to ways in which having a meaningful representation for a problem served students well as they solved combinatorial problems.

2.1.2.4 - Problem-solving strategies. A final theme that has arisen in the combinatorics literature is that of the utilization of problem-solving strategies, including verification strategies. In particular, Eizenberg and Zaslavsky (2004) have written about verification strategies that undergraduate students implemented as they solved combinatorial problems. In this study, the authors acknowledge that, generally, verification in combinatorics problems is difficult to accomplish. While the verification strategies are arguably somewhat general, the authors seek to relate them specifically to solving combinatorial problems. Eizenberg and Zaslavsky categorize five verification types (reworking the solution; adding justification to the solution; evaluating the reasonability of the answer; modifying some components of the solution; and using a different solution method and comparing the two), and they judge the relative effectiveness of the strategies. The authors note that while many strategies were generated by the students, the students were not always equipped to come up with (and, more importantly, to implement) efficient verification strategies on their own. English also suggests the 
importance of verification; she notes that, "quality of the children's checking actions played a crucial role in goal attainment" (1991, p. 451).

Another of Eizenberg and Zaslavsky's studies (2003) focuses on the effects of collaboration (in which students worked on problems in pairs instead of individually) and, in particular, the effects of control (i.e., metacognitive) processes. In their analysis, they examine control indicators that give evidence for particular metacognitive problemsolving activity. They found that collaborative problem solving seemed to be more effective than individual problem solving, but they note that "success in problem solving in combinatorics is not a direct outcome of collaborative problem solving. It is mostly a result of enhanced control behavior" (p. 399). That is, they believe that collaboration led to increased control, which in turn led to improved problem solving.

Glass and Maher (2004) also discuss problem solving in a combinatorial context, although their approach has a different emphasis than those mentioned above. That is, while in Eizenberg and Zaslavsky's work $(2003,2004)$ the emphasis is on problem solving as a means to facilitate success on a combinatorics problem, Glass and Maher focus on ways in which a rich combinatorics problem may foster productive problem solving skills. Indeed, they focus on justification and reasoning, and they argue for the value of a rich problem (which combinatorial problems tend to be) in the development of justification and problem solving strategies. In their study, Glass and Maher conducted a 
study on 150 students from a diverse background as they solved the tower problem ${ }^{3}$ (Maher, 2002). After analyzing the 150 students, four types of justification emerged, which Glass and Maher characterize as "justification by cases, inductive argument, elimination argument, and analytic method (use of formulas)" (p. 464). They expound upon these in their work and make the claim that a major implication of the study is that rich problems lead to better explanations and justifications.

In another take on the intersection between problem solving and enumeration, Mamona-Downs and Downs (2004) use the idea of creating bijections for purposes of enumeration as they study the development of techniques in problem solving. They designed three tasks that each involved combinatorial problems that could be solved by identifying a bijection between two finite sets, and they believed these tasks would elicit in their students the particular technique of forming such a bijection. Their findings indicated, however, that students generally did not make such bijections and chose alternative techniques to approach the problems.

There is thus an overwhelming sense that general problem solving strategies are inextricably linked with the successful solution of combinatorics problems. This makes sense, as solving counting problems is really a particular type of problem solving, but there is also a sense (as suggested by Eizenberg \& Zaslavsky, 2004) that some of these problem-solving strategies may be able to be described in a domain-specific way, relating

\footnotetext{
${ }^{3}$ The problem states: How many towers can you build, four high, selecting from cubes available in three different colors, so that the resulting towers contain at least one cube of each color? List all the possible towers. Justify that you have them all. (Glass \& Maher, 2004, p. 464).
} 
particularly to the solving of combinatorics problems. What we see from these studies listed here is that an ability to tackle and categorize problem solving strategies (especially any that may be particular to combinatorics) seems to be a relevant theme in the literature on combinatorics education.

To summarize this Literature Review, we have first seen a comprehensive look at the available studies that have been done on the teaching and learning of combinatorics, including studies in which combinatorial ideas were examined in the context of a variety of topics. We have seen from the combinatorics education literature a variety of noteworthy premises that include difficulties with counting, underlying structures, representations, and problem-solving strategies. While not all of these themes have a direct bearing on my research, they provide background into the kind of research that has been done thus far in combinatorics education. Additionally, it has been my intent to convey the need for further research on how students understand combinatorics and how they solve combinatorial problems.

\section{2 - Existing literature that pertains to my study}

While I have listed and discussed the available combinatorics education literature and have described some of the themes found among existing research, I now attempt to situate my study within this literature. In particular, while there are not many points of contact between my notion of set-oriented thinking and the current literature, I highlight those existing studies to which there is some connection to my work. In order to do this effectively, I must briefly describe some of the mathematics involved in my study. 
Although I will discuss a much more detailed conceptual analysis of the mathematical ideas in Section 4.1, in this section I offer a cursory look at the mathematics in order to better situate my study. While the precise focus of my study has shifted in the course of conducting the research, and while I have changed some of my ways of thinking about (and my definitions of) some of these mathematical constructs, there is value in giving a sense of where I began as I designed my study, and to see what mathematical constructs had direct effects on what I did.

Specifically, there are two major parts of this section. First, in 2.2.1 I discuss my early conceptions of set-oriented thinking (and process-oriented thinking), and I relate these ideas to mathematics described in both combinatorics textbooks and in the mathematics education literature. I also address how the set and process relationship arise in mathematics education more broadly. Then, in 2.2.2 I give details on the mathematics behind errors of overcounting which influenced both the selection of tasks and also some elements of the study's design. These mathematical ideas, and particularly their treatment in combinatorial textbooks and in some mathematics education studies, have informed design and methodological decisions.

\subsection{1 - A discussion of relevant mathematical notions as they relate to and inform set-oriented thinking}

Coming into the study, I was aware of a potential distinction between what I called set and process-oriented thinking (described below). My initial view of these ideas was formed by three major strands. First, my view was based on existing ideas documented 
both in combinatorial textbooks and in the mathematics education literature, as we will see. Second, it was based on my own experience as a teacher and student of combinatorics. Third, the distinction was borne out during a pilot study, which resulted in data that suggested that further investigation into set-oriented thinking might be worthwhile. Therefore, these three elements contributed to designing a study in which I sought to explore set and process-oriented thinking in counting problems. I discuss both of these kinds of thinking now in detail, with the intent of situating my work within the literature. I expound upon the mathematics in detail in Section 4.1.

When I was developing the study initially I was very focused on the distinction between set and process-oriented thinking. I had not fully articulated it, but I suspected that perhaps students engaged either in set-oriented thinking or in process-oriented thinking, and I had not yet given careful consideration to whether a person could consider both simultaneously. In fact, in looking back, I think that I had used the construct of process-oriented thinking as a way to show what thinking in absence of set-oriented thinking might look like. My stance on this has changed, however; I no longer see process-oriented thinking only as the absence of set-oriented thinking, and in fact I think that process-oriented thinking is quite important. I have very much shifted the focus toward set-oriented thinking, though; my study examines student's uses of set-oriented thinking and does not explicitly address process-oriented thinking.

2.2.1.1 - A set-oriented perspective. As I began the study, my initial notion of setoriented thinking was that, to the counter, the task of counting amounts to counting the number of objects in a set. Intrinsic in this is a conceptualization of the things to be 
counted as a set of objects - a set which a student could manipulate as they would any other set. This meant that they could conduct membership tests to determine elements in the set, they could consider subsets or partitions of the set, they may consider the union or intersection of that set with other sets, and so on. According to this way of thinking, then, any counting technique or procedure could essentially serve to organize this set of objects in some way, producing structure that often makes the enumeration of that set more manageable. As an example, a case breakdown is one such counting technique that serves to organize the set of outcomes. In a problem like, "How many times does the digit 7 occur in the list of positive integers from 1 to 9999 ?" the set of outcomes can be organized according to the number of digits the outcome contains. A useful case breakdown might be to consider the one, two, three, and four-digit numbers separately. This case breakdown partitions the outcomes, and each individual case can then be handled as its own counting problem which, in this example, is more manageable to count than the original counting problem. When I talk about conceiving of a counting problem in a set-oriented way, it is not the case that students need to be able to picture or produce an exhaustive list of every object to be counted; this may often not be possible. Rather, set-oriented thinking is characterized by the capacity to conceive of the act of counting as counting some set of objects and manipulate that set of objects as they would any other set.

2.2.1.2 - A process-oriented perspective. My initial idea of process-oriented thinking was that solving a counting problem is conceived of as a process of completing a task, which might be broken down into individual stages. In the problem, "How many ways can 15 
toys be distributed to five children?" a process-oriented perspective might elicit a solution that could be broken down into stages of a task - first we distribute the first toy to a child, then we distribute the second toy to another child, etc. There is an emphasis on the action, the act of distributing the toys, rather than the outcomes of the process, which is the resulting set of distributions. I conjectured that the process-oriented way of thinking may have allowed someone to arrive at a solution of a problem without ever having to think about manipulating some set of objects. It seemed that in some instances, this process-oriented way of thinking is something that students utilize as they become more familiar with counting formulas and procedures.

2.2.1.3 - Set and process-oriented thinking in combinatorial texts. In order to further articulate this distinction, I considered the presentation of counting in several combinatorics and discrete mathematics texts. To do this, I focused especially on these books' treatment of two main counting rules: the addition principle and the multiplication principle (called the sum and product rules, respectively, by some). The enumeration sections of many combinatorial texts (e.g., Bona, 2007; Brualdi, 2004; Roberts, 1984; Tucker, 2002) begin with a discussion of these principles, and texts that do not emphasize these initially (e.g., Cameron, 1994; Martin, 2001) clearly assume that the reader has facility with them. The focus on these two principles is well-founded, as they underpin the majority of subsequent counting rules, principles, and techniques. In this section I discuss the fact that the nature of the definitions of these principles vary within and across textbooks; the purpose of this discussion is to highlight how the distinction 
between set-oriented and process-oriented thinking arose for me as I examined these textbooks.

Tucker's (2002) treatment of the addition and multiplication principles had an impact on me, because I took his definition of the addition principle as decidedly set-oriented, while I took his definition of the multiplication principle to be process-oriented. He asks the reader to "Remember that the addition principle requires disjoint sets of objects and the multiplication principle requires that the procedure break into ordered stages and that the composite outcomes be distinct" (p. 170), and I felt that this summary illustrates the two different potential ways of thinking. To illustrate this, the exact statements of each principle as found in Tucker (p. 170, emphasis in original) are:

The Addition principle:

If there are $r_{1}$ different objects in the first set, $r_{2}$ different objects in the second set, ..., and $r_{\mathrm{m}}$ different objects in the $m$ th set, and if the different sets are disjoint, then the number of ways to select an object from one of the $m$ sets is $r_{1}+r_{2}+\ldots+r_{m}$.

Multiplication principle:

Suppose a procedure can be broken into $m$ successive (ordered) stages, with $r_{1}$ different outcomes in the first stage, $r_{2}$ different outcomes in the second stage, $\ldots$, and $r_{m}$ different outcomes in the $m$ th stage. If the number of outcomes at each stage is independent of the choices in the previous stages and if the composite outcomes are all distinct, then the total procedure has $r_{1} \times r_{2} \times \ldots \times r_{m}$ different composite outcomes.

Other authors vary in their treatment of these definitions. Roberts and Tesman (2005) define the two principles exclusively in a process-oriented way. Some authors (e.g., Brualdi, 2004; Rosen, 2007) list both a set-theoretic and a process-oriented definition of 
the principles. Rosen (2007), too, defines the product rule and the sum rule both in a process and a set-oriented way, opting to present the process way first.

While the above mathematical discussion was important for the development of my own thinking, I do not want to over-emphasize the importance of these particular definitions in my work. While they set the stage for what would become the focus of my study (set-oriented thinking), these definitions themselves were not the basis for what I would come to understand as set-oriented thinking. I present them as a motivation for the question of what set-oriented thinking might entail, and I point out that my thinking of the mathematics and of the notion of set-oriented thinking had its roots in the mathematics discussed above. The existence of such a distinction in combinatorics textbooks raised two questions for me. First, although these texts present two different definitions of fundamental counting principles, I wondered if they, in practice, translated to two different ways of thinking of counting that students may possess. In other words, the distinction between set-oriented and process-oriented perspectives in formal definitions of counting principles is clearly documented in combinatorial textbooks. The question was whether this distinction actually correlates to a difference in ways that students think about and solve problems, or whether this distinction is merely a matter of formal mathematical definitions. Second, I wondered if the distinction was useful in studying how students understand and know counting principles, and how they solve counting problems; that is, was this distinction somehow relevant to research on students' solving of counting problems? It was this latter question that ultimately led to the development of my research questions. 
While I have since shifted away from the distinction between set and process-oriented thinking (and instead on the ways in which students utilize set-oriented thinking as they count), this discussion of textbook definitions of the addition and multiplication principles was an important step in my research. That is, by looking at the distinction between sets and processes, I was led ultimately to focus on set-oriented thinking. I now discuss the presence of set-oriented thinking in the mathematics education literature.

\subsubsection{4 - Set and process-oriented thinking in the combinatorics education literature.}

The relationship between set-oriented and process-oriented perspectives is not so clearly defined in the mathematics education research literature on counting. If mathematics education researchers have noticed this distinction, they have for the most part have done so tacitly. Indeed, there does not seem to be any mention of the ways in which either set or process-oriented thinking may affect the ways in which students solve counting problems. What is present in the literature, though, is at least some evidence that setoriented ways of thinking are considered by some to be a natural way of thinking about counting. I examine three examples (English, 1993; Hadar \& Hadass, 1981; MamonaDowns \& Downs, 2004), in which counting is described, at times, in terms of what I would take to be set-oriented thinking.

Hadar and Hadass (1981) are frequently cited for their discussion of seven pitfalls that befall students as they count. Based on their work with students in a first course in combinatorics (they provide no specific data in their paper), they offer insight into the kinds of mistakes and hardships that their students faced when solving counting problems. The seven pitfalls are: identification of the set of events in question; choosing 
appropriate notation; perceiving the overall problem as a set of particular problems; construction of a systematic method; fixation of one (or more) of the variables; realization of the counting plan; and, generalization. They use an example of one counting problem in order to address each of these pitfalls. For my purposes, what is most noteworthy about this paper is its discussion of set-oriented thinking as it relates to counting. In their discussion, there is an assumption that solving a counting problem entails counting a certain set of objects or events. This gives credence to the above discussion of set-oriented thinking, providing an example of researchers who view counting in this particular way. This is evidenced in several places in the article. Although there are seven pitfalls, I will discuss the first four pitfalls here, as each of these include a direct reference to set-oriented approaches to counting.

Their first pitfall is entitled Identification of the set of events in question. They note that "in general, an incoherent perception of the set of events to be counted is misleading. It might bring out a solution to a question which was not asked" (Hadar \& Hadass, 1981, p. 436). This indicates that, to Hadar and Hadass, the solution to a counting problem is thought of as the cardinality of a set of events to be counted; failure to identify what set students are meant to count could lead to an incorrect solution. Their second pitfall is Choosing an appropriate notation. While this pitfall does not directly relate to sets, in their discussion of it they say that "a proper notation must not fail to reflect the fact that the (finite) disjoint sets of envelopes and letters are of equal size, and moreover, that there exists a specific one-to-one correspondence between them" (p. 436). This language of one-to-one correspondence again signifies an assumption that counting and set- 
oriented thinking are related. Hadar and Haddas' third pitfall is Percieving of the overall problem as a set of particular problems. In their discussion, they emphasize the importance of "perceiving the one problem as a set of particular problems. Recognizing this fact is vital. Without it one would not be able to think of examining particular cases, which is most often a breakthrough in the process" (p. 438). This connection between breaking the problem into a set of other problems and a case breakdown is an important one, and we see it underscored again in their discussion of the fourth pitfall, Construction of a systematic method. In their experience, many of their students failed to plan the counting in a careful way so as to ensure the counting of each and every possibility once and only once. Many times students formed subsets of possibilities to be counted, which were not mutually exclusive. Also, very often, the union of the partial sets counted did not coincide with the whole set under discussion (p. 438). Hadar and Hadass use the word "possibilities" to indicate various outcomes of the counting procedure - that is, the objects that are to be counted. Again, this language indicates that these authors assume there is an aspect of counting that is inextricably related to determining the cardinality of a set.

Another instance of set-oriented language arising in mathematics education literature is English's (1993) work. English's (1991, 1993) studies had young students count outfits for bears, and they worked with relatively small numbers of shirts and pants. Enumeration of elements of the solution set in these studies is perhaps different from enumerating sets which cannot realistically be listed or organized completely. However, it is noteworthy that English, a mathematics education researcher, notes a relationship 
between combinatorics and set-theoretic language. For example, English describes combinatorics as follows,

"In simple terms, combinatorics may be viewed as the operation of cross product. The cross product of two sets, $A$ and $B$, is the set of combinations obtained by systematically pairing each member of $A$ in turn with each member of $B$ " (p. 257).

English also notes the tendency in combinatorial texts to offer alternative ways of defining combinatorial principles, noting that

Combinatorics is also defined in terms of the fundamental counting principle, which asserts that if one task can be performed in $n$ ways and a second task can be performed in $m$ ways, then the number of ways of completing the two tasks is $m n$ (DeGuire, 1991, p. 59) (p. 257).

This is an instance in which set-oriented language is used by a mathematics education researcher to describe combinatorics. In addition, the acknowledgment of an alternative, process-oriented definition of the multiplication principle is noteworthy.

Finally, Mamona-Downs and Downs (2004) offer a paper on the development of techniques in problem solving, and in their work they use combinatorial problems as a context in which to study students' development of particular targeted techniques. Specifically, they gave students three combinatorial tasks, and the technique they wanted students to use was to create a bijection between the set in question and another set that was easier to count. While their paper does not talk about set-oriented thinking (and in fact the paper is more about problem solving techniques than counting), it is noteworthy that they seem to assume a set-oriented view of counting. Mamona-Downs and Downs explain their choice for the development of bijections between sets as their targeted technique, and they say the following. 
"Our study implements only one technique, but it represents well how mental processing of familiar knowledge is often not in a form conducive for application in problem solving. Someone might know that a bijection between two finite sets implies that the number of elements in each is equal, yet would not be able to convert this fact into a solving technique, i.e., if asked to find the number of elements of a given set, one possible strategy would be to seek for another, better understood set with which a bijection can be constructed" (p. 236).

I maintain that in their language above there is an assumption that students see a counting problem as enumerating the number of elements in a set (and that students could create a bijection between two sets of equal size). I mention this study, then, as an example in which the authors seem to view counting as enumerating the elements in a set, which I take to indicate set-oriented thinking.

\subsubsection{5 - Set and processes-oriented thinking in the mathematics education literature.}

In this section, I speak to the general relationship between sets and processes in mathematics education literature. There are two prominent places in which my interest in set-oriented thinking could be framed within the broader research on this topic - in research involving APOS theory, and in research involving structural and operational conceptualizations. I discuss them now as points of connection of my work to related areas of mathematics education research and to situate my theoretical constructs relative to common theoretical perspectives in the field. While I see these as being related to my work, I present them primarily as theoretical background for my ideas related to setoriented thinking. I did not use them explicitly in my analysis because my analysis ultimately examined the ways in which students used set-oriented thinking as they solved 
counting problems, not at the distinctions or relationships between set and processoriented thinking.

A number of researchers (e.g. Asiala, Brown, DeVries, Dubinsky, Mathews \& Thomas, 1996; Dubinsky, 1994; Dubinsky and McDonald, 2001) present work on a theory of learning called APOS theory (the acronym stands for actions, processes, objects, and schema). I briefly outline some of the aspects of this theory and discuss the ways in which sets and processes fit into this framework. Dubinsky and McDonald note that APOS theory "begins with the hypothesis that mathematical knowledge consists in an individual's tendency to deal with perceived mathematical problem situations by constructing mental actions, processes, and objects and organizing them into schemas to make sense of the situations and solve problems" (p. 2). Asiala et al. define an action as a "transformation of objects which is perceived by the individual as being as least somewhat external" (p. 10), and they say that when an action is repeated and reflected upon by a person, it "maybe be interiorized as a process" (p. 10-11). In a process, some action may be carried out without external stimuli, and it may be reflected upon by the person doing the process. Then, according to Asiala et al., when a person can consider operations being applied to that process and can conceive of the process as a totality, that person "is thinking of this process as an object. In this case, we say that the process has been encapsulated to an object" (p. 11, emphasis in original). Finally, the authors note that "a collection of processes and objects can be organized in a structured manner to form a schema" (p. 12). These researchers thus offer a theory of learning in which external actions can become processes, which can then be encapsulated to objects, and 
together processes and objects can be organized into a schema. In their discussion of these aspects of APOS theory, Dubinsky and McDonald note that while these aspects are described in a linear fashion, they may not be linearly constructed by students.

Additionally, Sfard (1991) makes an insightful and related distinction between what she calls structural and operational conceptions of mathematical notions. She notes that many mathematical concepts can be treated and described as objects, but they can also be discussed as processes, algorithms, and actions. She says that "seeing a mathematical entity as an object means being capable of referring to it as if it was a real thing....it also means being able to ... manipulate it as a whole, without going into details" (p. 4). Sfard insists that this is a complementary relationship, not a dichotomous one. She also traces some historical concepts such as number and function and their structural and operational treatments. A typical example of a concept that can be seen from structural and an operational point of view is the concept of function. A number of researchers (e.g., Kaput, 1992; Kieran, 1992; Sfard, 1992; White, 2009) highlight the idea that the concept of function may be considered as a process (a mapping), or as an object (a set of ordered pairs).

There thus seem to be existing aspects of mathematics education research that have explored relationships between processes and objects. Both APOS theory as put forth by researchers such as Dubinsky and McDonald (2001), and Sfard's (1991) notions of structural and operational conceptions thus provide theory in which I can contextualize my work. And, too, there are potential points of connection between my work on setoriented thinking and between these ideas, and I discuss such relationships as further 
avenues of study in the Conclusions chapter. The above discussion was designed to frame my own work within the broader scope of mathematics education literature, and while the above works were not influential in the design of my study, they may serve as avenues of further research to which I may draw connections.

In this section I have attempted to make the case that there is evidence that: a) some combinatorial texts define counting problems, principles and solutions both in a setoriented and a process-oriented way; b) some of the combinatorics education literature acknowledges set-oriented thinking as a legitimate way of viewing counting; and, c) the relationship between sets and processes seems to be a relevant relationship within math education literature that has been addressed in broader contexts than combinatorics. In spite of this, no study has yet explicitly addressed the significance that these two different perspectives may have on students' solving of counting problems. We now turn to a discussion of the second major mathematical aspect of the study - errors of overcounting. Again I attempt to frame my discussion of overcounting within the existing literature on combinatorics education.

\subsection{2 - A discussion of relevant mathematical notions related to errors of overcounting}

As I considered set-oriented thinking, and the ways in which such thinking could be leveraged in counting problems, errors of overcounting repeatedly emerged as a potentially relevant idea. This issue had its roots in my own experience as someone who had both taught and learned counting over the years. This idea was substantiated as well, 
both by the combinatorics education literature (Batanero, Navarro-Pelayo, et al., 1997; Mellinger, 2004) and in several combinatorial textbooks (e.g., Bona, 2003; Tucker, 2002). In addition, further evidence of the occurrence of this particular error was borne out in two previous studies (Lockwood, 2009; Lockwood, 2010). Therefore, the focus on these types of errors stemmed from personal experience (further underscored by personal communication with a combinatorialist), the research literature and combinatorial texts, and pilot study data. Below, I discuss both the research literature and some specific examples from the pilot study that affected my focus on this particular aspect of counting.

Overcounting is generally warned against in combinatorial texts, as it is one of the ways that a counting problem can be incorrect - one may arrive at an incorrect answer to the question by getting an answer that is too small (and thus undercounting occurred) or too large (and thus overcounting occurred). However, there are some particular ways in which overcounting can occur that are difficult to detect in a seemingly correct counting process. It is possible for a counting procedure to generate an overall answer that is too big, because some of the solutions which should have been counted once actually were counted more than once. However, this error can, at times, be virtually undetectable to students, and the associated erroneous counting process can seem to them to be logically sound. In this section, I seek to make two main points: 1) errors of overcounting do in fact arise for students in counting problems, and 2) set-oriented thinking could possibly be leveraged as a way to uncover and correct such errors.

There are some errors that are called "errors of order" in the mathematics education literature (Batanero, Navarro-Pelayo, et al., 1997; Mellinger, 2004). While these are 
described explicitly as errors of order in the literature, I consider these as exemplifying a particular way in which errors of overcounting can occur; that is to say, they fall under the umbrella of overcounting errors, and they will thus be addressed in this study. When this error of order occurs, students make mistakes or solve a problem erroneously because they somehow incorrectly 'order' their solutions - either they impose order when they should not, or they fail to impose order when their solutions should be ordered. Often this arises when the distinction between combinations and permutations is not correctly made. Batanero, Navarro-Pelayo, et al. (1997) list as a specific error an error of order, which they characterize as follows: "This mistake consists of confusing the criteria of combinations and arrangements, that is, distinguishing the order of the elements when it is irrelevant or, on the contrary, not considering the order when it is essential" (p. 191192). Their example of this error involves a student solution in which the student was asked to count selections of people, but instead he or she counted arrangements of people. The student's failure to see that the same group of people is actually being counted multiple times resulted in an overcount of the solution. The point of this example is that this issue of confounding permutations and combinations can lead to overcounting, and this is something that has showed up in the mathematics education literature.

Colloquially, this issue of order is often phrased as whether “order matters or doesn't matter." For example, Mellinger (2004) says that

"Another problem with counting arises when one is trying to select a subset of elements from a larger set. Ordering is the source of many problems. We have several scenarios. Does the order in which you must choose the elements matter? Does the order of the subsets matter?" (p. 334). 
When errors of order particularly relate to the conflation of combinations and permutations, the above questions by Mellinger can be addressed in a fairly straightforward way - order "matters" if we wish to count permutations, where we want to count different arrangements as being distinct solutions to the problem, and order does not "matter" if we desire to count selections, where we do not want to count different arrangements as being distinct solutions to the problem. In fact, the reader may note that these commonly used expressions of order mattering or not mattering can be particularly meaningful in the context of set-oriented thinking. It is precisely whether certain outcomes should be considered distinct or not that can answer a question of whether or not "order matters" in a problem.

Issues of order and overcounting are not always so clear as a mere distinction between combinations and permutations. Indeed, there are much more subtle ways in which overcounting can arise. Let us consider a question suggested as a classroom activity by Mellinger (2004). In the first example we will discuss, he lists two problems and asks his students whether the answers are equal:

"Problem 1: Ten students want to form 2 teams of 5 players each to play basketball. In how many ways can they form 2 such teams?

Problem 2: A woman would like to distribute 10 different candy bars to 2 trick-ortreaters. In how many ways can this be done if each child receives 5 candy bars?

Question: Is the answer to Problem 1 equal to the answer to Problem 2?" (p. 334)

The two problems do not yield the same answer, in spite of appearing to be worded very similarly. Mellinger (2004) explains the reason for their difference by noting that in Problem 2, one of the children may be named Sara, and her favorite candy bar might be 
Snickers (and we may suppose only one of the candy bars in the problem is a Snickers, as they are all different). He says,

Once the candy bars are broken into two sets of 5 bars each, there is still one important decision to be made, namely, does Sara get the set with the Snickers? In other words, the order of the subsets matters. We didn't have this problem with the two basketball teams (p. 334).

Here Mellinger says, "The order of the subsets matters," but we must ask ourselves what this phrase means in the context of this problem. Why should this argument convince us that the problems are different?

To shed light on this, I investigate the numerical difference between these two problems. There are $\left(\begin{array}{l}10 \\ 5\end{array}\right)$ ways of picking the first team, and $\left(\begin{array}{l}5 \\ 5\end{array}\right)$ ways of picking the second team from the remaining players, and, therefore, it appears that the use of the multiplication principle yields an answer of $\left(\begin{array}{l}10 \\ 5\end{array}\right) \cdot\left(\begin{array}{l}5 \\ 5\end{array}\right)$. However, this solution is actually too big; overcounting has occurred. To see why this happened, we consider a specific solution in order to argue this point. In Problem 1, suppose that the ten students consist of five boys and five girls, and, for simplicity's sake, suppose that the two groups are broken up into the group of boys and the group of girls, call them B and G. As it is, the groups are different from each other, but according to our counting procedure, we could have first chosen five children (say we picked the boys), and the second group would then consist of the girls; I'll call this solution BG. Alternatively, as another solution, when choosing the first five children initially, we could have first chosen the girls and then 
chosen the boys, resulting in a solution GB. According to the procedure I just described, we could get BG and GB and count them as distinct solutions - when, really, they are both the exact same way of splitting up the ten students (namely, into a group of boys and a group of girls). Indeed, each solution we obtained by the formula $\left(\begin{array}{l}10 \\ 5\end{array}\right) \cdot\left(\begin{array}{l}5 \\ 5\end{array}\right)$ actually is counted twice, and so the correct solution should be $\frac{\left(\begin{array}{l}10 \\ 5\end{array}\right) \cdot\left(\begin{array}{l}5 \\ 5\end{array}\right)}{2}$. In contrast, we consider Problem 2, and we suppose that Sara and Dan are the two trick-or-treaters. We could divide the candy up in a similar way, yielding $\left(\begin{array}{l}10 \\ 5\end{array}\right) \cdot\left(\begin{array}{l}5 \\ 5\end{array}\right)$, where we first choose five pieces to go to Sara, and we pick the remaining five pieces to go to Dan. In this case, distinguishing between groups of candy makes sense; we could say that Sara gets the first pile and Dan gets the second. Suppose that our two groups of candy are called 1 and 2. In this case, choosing 1 then 2 means Sara gets group 1 and Dan gets group 2. And, choosing 2 then 1 would in fact be a different solution - it would mean that Sara gets group 2 and Dan gets group 1. Thus, the answer is $\left(\begin{array}{l}10 \\ 5\end{array}\right) \cdot\left(\begin{array}{l}5 \\ 5\end{array}\right)$; we do not have to divide by 2 because we want 1-2 and 2-1 to be distinct solutions. Therefore, because BG and GB should not be counted as different solutions in Problem 1, but 1-2 and 2-1 should be counted as different solutions in Problem 2, the two problems yield different answers. This is quite subtle, but it should illuminate the ways in which an apparently logical procedure could, surprisingly, lead to a solution that is too big. 
As a final example that addresses a similar issue of overcounting, we consider another problem (taken from Martin, 2001) from the pilot study ${ }^{4}$ : How many different nonempty collections can be formed from five (identical) apples and eight (identical) oranges? One way of solving this problem is the following: We can choose zero to five apples (six possibilities) and zero to eight oranges (nine possibilities); we cannot choose zero of each, so there are $6 \cdot 9-1=53$ collections. In the pilot study, students solved this problem in a variety of ways, but we present now an answer by Holly. Holly tended to work quickly through problems, and if she missed problems it was often because she did not read the problem carefully or consider what she was trying to count. Likely because the problem was not immediately recognizable to her as a certain problem type, the statement of this problem caused her to approach it a bit more carefully, and she drew a diagram like the one in Figure 1 below. She represented the respective numbers of apples and oranges she could have (one through five apples and one through eight oranges), and she began to reason through the solution. She drew eight branches from each row of A's - one branch each to one through eight O's. Similarly, she drew one branch from each row of O's to each of one through five A's.

\footnotetext{
${ }^{4}$ I discuss the pilot study in more detail in Section 3.1 of the Methodology chapter.
} 
A

AA

AAA

AAAA

AAAAA

$\mathrm{O}$

$\mathrm{OO}$

$\mathrm{OOO}$

OOOO

OOOOO

000000

OOOOOOO

OOOOOOOO

Figure 1

Initially, Holly used her diagram to arrive at an answer of $5 \cdot 8+8 \cdot 5+5+8=93$. The $5 \cdot 8$ is the number of ways she could have one to five apples with one to eight oranges, and the $8 \cdot 5$ is the number of ways she could have one to eight oranges with one to five apples. The 5 and 8 are the number of collections of one piece of fruit (apples and oranges, respectively). The problem with this solution is that the $5 \cdot 8$ and 8.5 both count exactly the same subset of solutions - she has counted the solution three apples and two oranges in the $5 \cdot 8$, but she also counted two oranges and three apples in the $8 \cdot 5$. Thus, overcounting occurred in Holly's initial solution. However, in this problem, Holly was able to look back and recognize this overcounting. After she reviewed her work for a bit, the following exchange took place as she referred back to her diagram; note that she detects her double counting by recognizing that a certain outcome was counted more than once. 
$\mathrm{H}$ : Oh am I double counting though? ... Oh I am, I'm way double-counting, so that number is way too big, because I'm getting one apple and one orange here, and one apple and one orange here...

E: Okay, and can you explain how the double counting was coming in? What was happening?

H: If I look at one orange and one apple, it's the same as one apple and one orange. [refers to Figure 1]

I: Okay, but you'd already counted that.

$\mathrm{H}$ : And I'd already counted that, and I was going to do that a lot of times.

The preceding excerpt suggests that Holly was able to detect double counting by appealing to a particular outcome (one apple and one orange) that had actually been counted twice. By acknowledging which collections had been over-counted, she was able to adjust her answer accordingly. This is a case in which being able to think in a setoriented way (by appealing to a particular solution) apparently enabled Holly to detect and fix her error of overcounting. I suspect that a student who had not used set-oriented thinking might not have: a) recognized her error; and, more importantly, b) recognized how to fix her error. In this example, I felt that Holly utilized set-oriented thinking to address an error, and this kind of thinking is indicative of the type of results $\mathrm{I}$ hoped to see as I designed my study. I wanted to observe ways in which students drew upon setoriented thinking as they solved counting problems in order to deepen my understanding of students' counting activity.

\section{3 - Summary of the Literature Review chapter}

This chapter had two major components. First, I gave a full review of the mathematics education literature on combinatorics, which included a discussion of 
relevant themes and ideas. Then, I discussed the mathematics in which my study is rooted, connecting the mathematical ideas to existing mathematics education research. This included the development of my ideas regarding set-oriented thinking (and its presence in combinatorics textbooks and the mathematics education literature), as well as detail about errors of overcounting that have played into the design of the study. 


\section{Chapter 3 - Methodology}

In this chapter, I lay out the details of the implementation of the study, including the specific research methodologies I used. In order to give some motivation for the design, I will first discuss the pilot study, which shaped some of the design decisions, and I will then discuss the overall design of the study. Then, I will give details on data collection, including a description of and a rationale for the interview tasks, and finally I will elaborate details of the data analysis.

3.1 - A discussion of the pilot study

3.2 - Description of, and rationale for, the overall design of the study

3.3 - Subject selection

3.4 - Data collection

3.5 - Data analysis

3.6 - Summary of the Methodology chapter

\section{1 - A discussion of the pilot study}

Throughout the dissertation, I have at times referred to a pilot study that helped inform some of my design decisions. In this section, I share several more details of this pilot study in an effort to provide additional motivation for certain methodological decisions which will be outlined in this chapter. In particular, I discuss specific lessons that I learned from the pilot study which had a direct influence on the design of the main study. 
Lockwood Dissertation - 51

\subsection{1 - A brief synopsis}

The pilot study was conducted in January and February, 2010. I distributed surveys to three different classes: an introductory statistics course for non-math majors; a juniorlevel discrete mathematics course; and a history of mathematics course designed for senior-level math majors and graduate students, aimed at prospective high school teachers. I selected two students from the statistics course (one student discontinued participation after one interview), three from the discrete mathematics course, and two from the history of mathematics course. I interviewed the remaining six students three times each, and the data consisted of nineteen videotaped interviews, each approximately one hour in length. The students were interviewed separately to get a sense of how the individuals thought about and solved counting problems. In the interviews, the students were given several counting problems (they completed anywhere from four to eight in the hour), and the protocol for the interview sessions was to allow the students to work on the problem until they felt they had finished. Once the students were done, I returned to the problem and asked them to explain their activity. Therefore, the data includes the work that students did when left on their own, and it also incorporates some explicit discussion and explanation by the students as I questioned them more specifically about their activity.

Analysis of this data consisted of re-watching the videos and making content logs while doing so. In writing these content logs, I included time stamps of relevant problem solving activity and highlighted particularly relevant mathematical issues that arose. Next the content logs were reviewed for salient themes, and, based on these emerging themes, 
several relevant sections were transcribed. Through the analysis of the pilot study data, I sought to gain insight about possible methodological lessons that I might learn for a subsequent dissertation study. Although the pilot study was actually initially designed around the investigation of the addition and multiplication principles, it was the notion of students' language about sets (and particularly the set of outcomes) that emerged for me as being the most interesting feature of the students' mathematical activity, as well as being the element with the most inherent research potential. This idea of focusing on what I would later call students' set-oriented thinking had arisen in a conceptual analysis of the addition and multiplication principles, but throughout the pilot study it emerged as the particular feature that I desired to investigate in my dissertation.

\subsection{2 - Lessons learned from the pilot study}

Although some interesting examples of student work emerged during the pilot study, the pilot study did not provide enough data to sufficiently explore what might be involved in students' consideration of the set of outcomes (and at that point I was more focused on a distinction between set and process-oriented thinking). Rather, the value of the pilot study was to provide me with a handful of methodological lessons that I could apply when I designed my dissertation study. I have alluded to one specific instance from my pilot study which exemplifies the types of results that emerged from the pilot study (an example involving Holly, as discussed in Section 2.2.2), but beyond this I do not include any other specific data from my pilot study (the reader may refer to Lockwood (2010) for 
further details). I discuss now the specific ways in which the pilot study has influenced the methods described throughout the rest of this chapter.

As a result of the pilot study, I was able to identify appropriate populations of students. I learned that the students in a lower-division statistics class whom I interviewed did not have the combinatorial background I desired to address the kinds of combinatorial issues I was curious about. While some interesting examples emerged from interviews with introductory statistics students, for the most part I felt restricted in what I could ask them, and also in what I could ultimately glean from them regarding setoriented thinking. Even more, I came to realize that the kinds of errors of over-counting that I desired to focus on required a certain facility with counting. That is, in order for me to investigate ways in which student's set-oriented perspectives might arise, I needed students to be familiar enough with counting to have some counting techniques and strategies in place. Therefore, I decided to focus on mathematics majors, particularly those who had certain facility with some basic combinatorial ideas (such as binomial coefficients) and who possessed some counting heuristics.

In the pilot study, I was focused on the addition and multiplication principles. I had some sense that set-oriented thinking might be a topic of importance, but I did not know how to address it specifically in the pilot study. My questioning strategies were quite broad, and I spent three interviews with each participant, essentially watching them solve counting problems. It was a useful experience to observe students solving counting problems naturally and of their own accord, but I was not very focused - not in my interviewing protocol nor in my task selection. The tasks I chose were broadly geared at 
addressing the addition and multiplication principles, but ultimately this allowed for much interview time to pass without a clear sense of specific mathematical direction. Therefore, in designing the dissertation study, I learned that I ought to tighten up the interview process, both in terms of the mathematical focus and the interview protocol. From this insight, the emphasis on errors of over-counting emerged as a particular, narrow mathematical aspect of counting problems that I decided to pinpoint. Therefore, instead of conducting multiple interviews with students doing a wide variety of problems, I attempted to streamline the process by posing counting problems that were susceptible to errors of over-counting 5 .

Additionally, related to this, I also learned that my interviewing strategy in the pilot study could be made more effective. As mentioned above, the general strategy during the interviews was to let the students work on problems autonomously. In an effort to create a situation where I could observe how students naturally solve counting problems, I intentionally did not teach or guide them, nor did I indicate that they should go back and re-work an incorrect problem. In retrospect, this was a useful strategy in some ways (particularly because it allowed me to observe students' unadulterated approaches to counting problems), but it also caused me to pass by opportunities related to students' set-oriented thinking. In particular, I believe that, in some cases, if I had told students that their answer was wrong, and students had been forced to go back and detect and fix an

\footnotetext{
${ }^{5}$ There were deeper issues than overcounting; see Sections 3.4.1 and 3.4.2 for other factors that played into the task selection.
} 
error, I might have seen more evidence of students examining the set of outcomes. I thus wanted to put student in situations in which they would have to check and monitor their own work.

Finally, while the data did not provide overwhelming insight into students' setoriented thinking, I did find some examples to legitimize my sense that set-oriented thinking might be important. I have discussed one such instance in detail (Holly's case). At the very least, this example served to a identify point of departure from which to proceed with the study. Therefore, in summary, the methods detailed in the coming sections were directly influenced in part by my experience with the pilot study.

\section{2 - Description of, and rationale for, the overall design of the study}

\subsection{1 - A brief description and timeline of the research}

The general data collection method was to interview students in individual, videotaped sessions as they solved counting problems. This interview process consisted of two major stages, as described in Table 1 below. In Stage 1, I interviewed a smaller group of 8 students in two separate interviews each. I then analyzed the data from Stage 1 and, from this first stage of interviews, developed preliminary hypotheses (which could be examined in Stage 2). Then, in Stage 2 I interviewed 14 students in one interview each. The majority of the analysis then took place after Stage 2, when I did deeper analysis on the entire set of data. I will expound upon the particulars of what occurred at each stage below. 


\begin{tabular}{|c|c|c|c|c|}
\hline & Stage 1 & & Stage 2 & \\
\hline $\begin{array}{c}\text { Subject } \\
\text { Selection }\end{array}$ & $\begin{array}{c}\text { Two Rounds of } \\
\text { interviews, } \\
\end{array}$ & $\begin{array}{c}\text { Analysis of } \\
\text { Stage 1 }\end{array}$ & $\begin{array}{c}\text { One Round of } \\
\text { interviews, } \\
14 \text { people }\end{array}$ & $\begin{array}{c}\text { Reflective } \\
\text { analysis }\end{array}$ \\
\hline June 2010 & July 2010 & August - & December, 2010 & January - \\
& & October 2010 & & May, 2011 \\
\hline
\end{tabular}

Table 1

\subsection{2 - Further description and rationale of the overall design of the study}

My research questions are focused on specific mathematical activity, and in particular I have examined both the conceptual ways that students think about problems and the activities in which students engage when solving problems. It is impossible to know exactly what another person is thinking, and studies such as this rely on the physical, observable evidence that can point to students' thinking (e.g., particular phrases, drawings, representations, actions, etc.). Therefore, my data collection needed to utilize a method in which external evidence of student thinking could be observed. Indeed, the pilot study revealed numerous instances in which it proved invaluable not only to hear the exact utterances that subjects made, but also to see their corresponding written work and gestures as well. Because of this, I conducted videotaped interviews, in which students' utterances, gestures, and written work were captured and later analyzed. The interviews put students in a situation which generated rich data in ways that other methods, such as gathering surveys, could not. I chose to videotape (and not only audiotape) because it 
allowed me to revisit and re-examine what transpired during the interviews. Powell, Francisco, and Maher (2003) offer a review of the literature on the collection of videotape data. They say that "video is an important, flexible instrument for collecting aural and visual information" (p. 407), and they cite Clement (2000) when they note that video is able to capture "rich behavior and complex interaction and it allows investigators to examine data again and again" (Powell et al., 2003, p. 407). In addition to this, interviews were an appropriate method because interviews allowed flexibility in the data collection process. While there was an intentional protocol to each interview (Section 3.4.2 below), the interactive interview methodology enabled me to adjust and finesse questions throughout a given interview. Additionally, during an interview I could ask probing questions to try to further elicit the interviewee's thinking. Depending on the particular interviewee or on the particular problem, there were times in which I had to make slight adjustments, and the interview setting made such flexibility possible.

In Stage 1, I interviewed eight students (details of subject selection are in Section 3.3). The rationale for this number was to provide a small enough number for which the interviews could realistically be analyzed (by one person in a matter of months) to a level of detail where meaningful insights could be drawn and hypotheses could be formed. I interviewed each of these eight students twice. In the first interview I gave these students five to seven problems; they solved the problems autonomously, and when they were done we discussed their work. In the second interview I gave the students alternative answers to some subset of the problems they had solved and asked the students to make 
sense of the new expression (more details of the interview protocols, including rationale for this particular aspect of the design of the study, are described below in Section 3.4.2).

In Stage 2, I wanted the number of students to be large enough so that I could achieve theoretical saturation (Auerbach \& Silverstein, 2003). The idea behind theoretical saturation is to continue to interview participants "until you find that new groups of participants are not producing new data that add new concepts to your theory" (p. 19). In Stage 2, I interviewed fourteen students (after which point I felt the data was saturated), and I was able to streamline the interviews substantially. I condensed both their problem solving and their examination of alternative answers into one interview. Thus, in Stage 1 I went deeper with a few students, and in Stage 2 I was able to cover more ground with a larger number of students.

\section{3 - Subject selection}

While I wanted to explore student thinking about counting problems, I was not interested in measuring their learning. Thus, while I strove to be informed about the students' combinatorial background, I did not need to control for their previous knowledge and experience in order to gauge their progress.

I had used a counting survey to recruit students in the pilot study, and the pilot study had revealed that students' performances on the survey did not always correspond with students' abilities to count. In fact, what seemed to be most important in subject selection in the pilot study was the participants' own willingness and interest in being involved, which seemed to facilitate the recruiting students who were willing to talk and explain 
their thinking. Based on the types of counting errors and issues I was interested in, I sought a certain level of mathematical maturity among the subjects of this study. In particular, the pilot study revealed that knowledge of binomial coefficients (such as an ability to use and properly manipulate the "choose notation") was a valuable tool for the pilot study participants, and thus on the survey I included questions that had solutions involving binomial coefficients. My subject recruitment for both stages of the dissertation was fairly similar. In the recruitment process, I went into certain mathematics classrooms (discussed in detail below), and I asked the students to complete a counting survey (see Appendix A). In addition to mathematical content, I included a question on the survey which asked participants about their level of interest in being involved in the study, and I took this into account as I selected students.

I drew upon two populations of post-secondary students - mathematics majors who were enrolled upper-division mathematics courses, and prospective or in-service high school teachers enrolled in senior-level courses. In Stage 1, I recruited students from four classes being taught in Summer, 2010 - Number Theory, Advanced Calculus, Modern College Geometry, and a Probability for Teachers class. The rationale behind recruiting from this group of students was to choose students who might have some facility with counting so I could present them with interesting tasks, as discussed above. In Stage 1, I successfully recruited two computer science majors, four senior-level mathematics majors, and two post-bac students who were preparing for graduate work in the fall (one had a bachelors degree in mathematics, one had a $\mathrm{PhD}$ in chemistry). 
In Stage 2, I recruited in Graph Theory and Set Theory classes, and I chose 14 students. One result of the analysis of Stage 1 (discussed in Section 3.5.2) was that I felt I should recruit some stronger students. Therefore, as I recruited for Stage 2, I was open to recruiting not only undergraduate mathematics majors, but also graduate students as well. In Stage 2 I recruited 14 more students - one junior math major, six senior math majors (one of whom was a returning post-bac), and seven graduate students ${ }^{6}$. Table 2 below gives a list of all 22 students' pseudonyms and their major at the time of the interview (the Stage 1 students are listed first, then the Stage 2 students).

\begin{tabular}{|l|l|l|}
\hline Stage & Student & Academic Status \\
\hline 1 & Anderson & Senior level computer science major \\
\hline 1 & Casey & Senior level computer science major \\
\hline 1 & Daniel & Senior level mathematics major \\
\hline 1 & Jenny & Post-bac mathematics major, entering MS in statistics program \\
\hline 1 & Kristin & Senior level mathematics major \\
\hline 1 & Nancy & $\begin{array}{l}\text { Has a PhD in chemistry, taking courses to prepare for graduate } \\
\text { work in mathematics }\end{array}$ \\
\hline 1 & Nick & Senior level mathematics major \\
\hline 1 & Mike & Senior level mathematics major \\
\hline 2 & Aiden & Senior level mathematics major \\
\hline 2 & Ben & MS in mathematics student \\
\hline 2 & Brandon & Senior level mathematics major \\
\hline 2 & Jon & Senior level mathematics major \\
\hline 2 & Joshua & MS in mathematics student \\
\hline 2 & Keith & Senior level mathematics major \\
\hline 2 & Kim & PhD in mathematics education student \\
\hline 2 & Makaena & MS in mathematics student \\
\hline
\end{tabular}

\footnotetext{
6 The graduate students' programs are as follows: One student was in the mathematics education PhD program, one was in the applied mathematics $\mathrm{PhD}$ program, four students were in the master's in mathematics program (MS), and one was in the master's in teaching mathematics program (MST).
} 


\begin{tabular}{|l|l|l|}
\hline 2 & Marcus & Junior level mathematics major \\
\hline 2 & Matthew & MS in mathematics student \\
\hline 2 & Owen & PhD in mathematical sciences student \\
\hline 2 & Paige & MST in mathematics student \\
\hline 2 & Peter & Senior level mathematics major \\
\hline 2 & Zach & MS in mathematics student \\
\hline
\end{tabular}

Table 2

\section{4 - Data collection}

As I have articulated in the Literature Review chapter, I chose problems that focused on errors of over-counting that might highlight students' uses of set-oriented thinking. The mathematical content of the interview tasks will be outlined below. However, I will first speak about the structure and design of the interviews themselves.

\subsection{1 - Types of interview tasks}

There were two broad types of activities in which students engaged, both of which served to put students in situations where they could articulate their thinking. The first task type was simply to have students attempt to solve problems, and, once they were done, to have them explain what they did. This protocol conferred the benefit of: a) providing me with access to students' natural solving of counting problems; and, b) offering additional access to their thought process by having them explain their steps and answer probing questions about their solutions. This was a fairly natural way to observe what students knew and how they thought about and solved counting problems. 
The second task type was to have students consider alternative answers/expressions ${ }^{7}$ to counting problems. In my case, this technique provided further opportunities for the subjects to talk about how they view and/or analyze the problem. And, more specifically, it allowed me to introduce solutions that I suspected would put in situations in which they were presented with seemingly equivalent processes that were actually different. I wanted to see how they would handle such situations, and particularly to see if they would refer to the set of outcomes in order to explain the differences between the processes. Essentially, being able to suggest alternative expressions allowed for further opportunities for student discourse about a problem, and even more it allowed me to gain insight about their utilization of set-oriented thinking. The decision to incorporate this kind of task was influenced by a desire to put students in a situation in which they would have to scrutinize their work and to assess the correctness of their own work and of alternative solutions.

\subsection{2 - Interview protocol}

I designed the interview protocol to allow for much flexibility; regardless of a student's initial answer, I was able to give them an appropriate alternative expression to evaluate. For each of the tasks (discussed in detail below) I had prepared a typical correct answer, as well as a common incorrect answer that involved overcounting in some way. Thus, by giving students alternative answers, I was able to ensure that they would analyze

\footnotetext{
${ }^{7}$ I refer to expressions to mean those combinatorial expressions that represent an answer to a counting problem. I will discuss these at length in the Results chapter, but I assume that the expression I presented to students represented not only a numerical expression but also a counting process that they could evaluate.
} 
solutions about which I was particularly curious. That is, if a student answered a question correctly initially, I could give them an incorrect answer that overcounted, to see if they could make sense of why it overcounted. Or, if a student initially got an incorrect answer, I could show them a correct one and have them make sense of it. And, because I suspected that set-oriented thinking might arise for students as they compared counting processes that differed in subtle ways (thus engaging in the checking phase of problem solving), it was important for me to ensure that students could, at some point, consider such differing solutions. My protocol allowed for students to evaluate common answers to the problems. The specific protocol was as follows:

1) The student solved a counting problem on their own

a. I posed a counting problem to the student, and I typically observed them working on it until they felt they had finished the problem. There was some time limit, as the interviews were capped at 90 minutes, and I occasionally had the student stop and move onto a new task in order to maintain the flow of the interview to ensure they had time to work on the remaining problems.

b. Once the student felt they were done, I asked them to talk back through their solution, explaining their work and what they did. This exchange was fairly interactive, and I asked questions to uncover any unclear aspects of the subjects' solution or explanation.

c. This same protocol was repeated for all of the problems the students attempted. 
2) Evaluating alternative expressions

a. I revisited some subset of the problems (typically 2-4) with each student. The number of problems and the particular problems we revisited depended both on time constraints and on their original work on the problems. Typically I prioritized the Passwords, Test Questions, and Groups of Students problems, and I sought to revisit problems that I felt could yield results about the student's set-oriented thinking. During this time, I asked the student to make sense of the proposed answers and to determine their veracity.

b. I then asked the student questions to clarify their statements and explanations. On some occasions during this part of the interview, I also intervened and told them whether they were correct or not (this did not happen with every student on every problem, but there were times in which I told the student which answer was right). This was done for a couple of reasons. In some cases, a student was stuck or said they did not know how to continue. Rather than stop their work on the problem, I occasionally intervened to keep them talking and to allow them still to engage in other aspects of the problem. Other times, I felt the need to speed the process along in order to have time for other problems. I may have felt that the student might eventually have been able to make progress on the problem, but I wanted to more quickly have them be in a 
situation where they could explain why an answer was right or wrong. Thus on occasion I told students whether or not an answer was correct.

In order to keep the students from suspecting that they were correct or incorrect based on my response, I used the same protocol for each problem I posed, and students attempted all of the problems before I proceeded with alternative answers. The rationale for this was to try to preserve students' natural approaches to a problem. That is, if they were shown alternative solutions or were asked to detect errors after their work on the first problem, it may have affected the way they solved subsequent problems. Therefore, this protocol necessitated flexibility on my part. Particularly in the Stage 2 interviews, when both original work and the evaluation of alternative answers were in one interview, I needed to be able to adjust the questions I asked students based on their initial responses to the problem; an appropriate alternative answer to present to them depended on their initial answer. For each task, then, I had at least two potential student solutions that I could use. In some cases I improvised and tried new solutions that I had not prepared beforehand. And, ultimately, I tried to be as flexible as possible, trying more than anything to get students to think and talk about their work on the problems. I now present the tasks used in the interviews.

\subsection{3 - Specific interview tasks with rationale}

There were numerous aspects of counting that I could have focused on in this study. Even after narrowing to an emphasis on set-oriented thinking, there are many possible topics, problem-types, errors, or misconceptions that could be emphasized. As discussed 
in the Literature Review chapter, I chose to focus on problems that would get at students' set-oriented thinking, and this emphasis on errors of over-counting and on comparing seemingly similar processes stemmed from my own personal experience (further underscored by personal communication with a combinatorialist), and from research literature and combinatorial texts (e.g., Batanero, Navarro-Pelayo, et al., 1997; Bona, 2005; Mellinger, 2004; Tucker, 2002), from pilot study data (Lockwood, 2010). I explain below how the chosen interview tasks relate to these particular issues.

My own experiences (the pilot study confirmed this) seemed to indicate that students might naturally tend to try to solve counting problems primarily without relying on setoriented thinking. It is difficult to substantiate this idea in the literature, because the literature has largely not offered insight into students' perspectives on counting problems (such as ways of thinking about counting), and certainly not about set-oriented thinking. In Section 2.2.1, I have discussed some of the limited ways in which set-oriented thinking has shown up in the mathematics education literature, but these largely did not have an effect on my choice of problems. The tasks I chose were adapted from Martin (2001) and Tucker (2002) and were influenced by my experience as a teacher and student of combinatorics. My goal was to select problems that might be solved without set-oriented thinking, but for which I could easily produce alternative solutions or particular errors that might encourage students to consider the set of outcomes (and thus set-oriented thinking).

In the following section I will describe the interview tasks in detail. The mathematical explanations below should provide some rationale for the inclusion of the problem - each 
incorrect answer highlights an error of over-counting, and the errors in the incorrect answers can be effectively explained by providing a problematic element of the set of outcomes - but I will discuss some aspects of the rationale now. To be specific, I chose these tasks because I thought they would generate particularly interesting data when students were in situations in which they were comparing alternative answers. From my experience, these were problems for which students could be convinced that an incorrect answer was actually correct, because the incorrect solution could sound convincing. And, when students learned that the answer was actually incorrect, the error could be effectively explained by appealing to the set of outcomes - in particular by identifying some outcome that was counted more than once (or not counted at all) by the incorrect expression. I was also influenced in this decision by a desire to facilitate students' checking phase of problem solving. I conjectured that if I could put students in situations in which they had to compare correct and incorrect solutions to these problems, I suspected that they would potentially draw upon the set of outcomes in order to explain the discrepancy. That was a major motivation behind the choice of these particular problems.

When I interviewed the students in Stage 1, I posed a total of seven problems. Upon analysis of the first stage of interviews, I decided to ask the students in Stage 2 five ${ }^{8}$ of those seven questions (I further explain this decision in Section 3.5.2.4 below), and the data I have analyzed carefully consists of all 22 students' work on these five problems. I

\footnotetext{
${ }^{8}$ Task 1 and 2 were adapted from Tucker (2002). Tasks 3, 4, and 5 were adapted from Martin (2001).
} 
now describe these five tasks in detail. I explain one or two correct solutions to the problem $^{9}$, and in some cases I discuss a common incorrect solution. I also provide rationale for the inclusion of the task and describe the alternative expressions I showed to students throughout the interviews.

\subsubsection{1 - The Passwords problem}

The Passwords problem states, "A password consists of 8 upper case-letters. How many such 8-letter passwords contain at least 3 E's?" The at least constraint is a tricky one. Because of this constraint, the problem can be broken down into cases, in which the passwords contain three, four, five, six, seven, or eight E's. For any of those cases, the number of passwords containing $k$ E's is found by choosing spots for those E's to go (done in $\left(\begin{array}{l}8 \\ k\end{array}\right)$ ways), and then filling in the remaining $8-k$ spots with any of the 25 letters that are not E. Therefore, the correct result is

$$
\sum_{k=3}^{8}\left(\begin{array}{l}
8 \\
k
\end{array}\right) 25^{8-k}=\left(\begin{array}{l}
8 \\
3
\end{array}\right) \cdot 25^{5}+\left(\begin{array}{l}
8 \\
4
\end{array}\right) \cdot 25^{4}+\left(\begin{array}{l}
8 \\
5
\end{array}\right) \cdot 25^{3}+\left(\begin{array}{l}
8 \\
6
\end{array}\right) \cdot 25^{2}+\left(\begin{array}{l}
8 \\
7
\end{array}\right) \cdot 25^{1}+\left(\begin{array}{l}
8 \\
8
\end{array}\right) \cdot 25^{0}
$$

Another correct approach is to subtract the "bad" cases from the total number of passwords - that is, to subtract from all 8 -letter passwords those that contain either 0,1 , or 2 E's. This answer yields the expression

\footnotetext{
${ }^{9}$ There are multiple ways of expressing correct solutions to a problem. The solutions I provide in this section are most relevant to what the students did and to the discussion at hand.
} 


$$
26^{8}-\left[\left(\begin{array}{l}
8 \\
2
\end{array}\right) \cdot 25^{6}+\left(\begin{array}{l}
8 \\
1
\end{array}\right) \cdot 25^{7}+\left(\begin{array}{l}
8 \\
0
\end{array}\right) \cdot 25^{8}\right]
$$

There is also a tempting solution that does not involve a case breakdown which reflects a common error. Namely, we could arrive at an answer of $\left(\begin{array}{l}8 \\ 3\end{array}\right) \cdot 26^{5}$ by arguing that by first choosing where to put three E's (done in $\left(\begin{array}{l}8 \\ 3\end{array}\right)$ ways), we are guaranteed to have at least three E's in the password. Therefore, the remaining five letters could be any letter, including an $\mathrm{E}$ (hence, $26^{5}$ ). However, the problem with this answer is that some particular solutions get counted more than once. For example, the solution $\underline{E} \underline{E} \underline{\mathrm{E}} \underline{\mathrm{A}} \underline{\mathrm{B}} \underline{\mathrm{E}} \underline{\mathrm{E}}$ $\underline{E}$ gets counted multiple times, both when the first three E's were chosen ( $\underline{E} \underline{E} \underline{E}{ }_{-}--_{-}$ ) and the rest of the word was filled in with $\mathrm{ABEEE}$, and then again when the last three E's were chosen $\left({ }_{-}{ }_{-}-\underline{E} \underline{E} \underline{E}\right)$ and the rest of the word was filled in with EEEAB.

I have chosen this task because it represents a type of problem that contains an at least constraint, where an overcounting error can easily occur. More specifically, the at least constraint can allow for a seemingly correct answer which does not rely on cases, but which involves just a two-step process (represented by the expression $\left(\begin{array}{l}8 \\ 3\end{array}\right) \cdot 26^{5}$ ). That is, the incorrect answer described above can seem correct (and indeed this was the case for the students), but the error could be explained by identifying a problematic outcome, as above. This was exactly the type of situation I wanted my students to experience, and this problem lent itself well to that. Additionally, passwords are a fairly common context 
for counting problems, and I wanted to have at least one problem that would provide a familiar situation for students. I suspected that while the context might be familiar, the protocol of having to evaluate an alternative answer might put students in a situation in which they had to monitor their work. Finally, I had used a problem similar to this in my own teaching, and, in my experience, it yielded rich discussion about issues of overcounting.

\subsubsection{2 - The Cards problem}

The Cards problem asks, "How many ways are there to pick two different cards from a standard 52-card deck such that the first card is a face card and the second card is a heart?"A correct solution to this problem involves a case breakdown. The number of choices available for the second card (a heart) is dependent upon whether or not the first card (a face card) is a heart. Therefore, the solution can be broken into cases based on whether or not the first card is a heart. In the first case, if the first card is a face card and a heart (three options), then there are twelve hearts left in the deck; there are thus $3 \cdot 12=36$ such pairs. In the second case, if the first card is a face card but not a heart (nine options), then there are thirteen hearts left in the deck, and there are $9 \cdot 13=117$ such pairs. Therefore, there are $36+117=153$ total pairs. A tempting incorrect solution is simply to apply the multiplication principle, arriving at $12 \cdot 13$ because there are twelve choices for the first card (twelve face cards) and thirteen choices for the second card (thirteen hearts). However, this over-counts the correct number of solutions. In particular, a pair such as the Jack of hearts and the Queen of hearts gets counted more than once. 
This task was included because its particular constraint (the two specifications in the problem are not independent) means that it cannot be solved simply with a single application of the multiplication principle. However, the problem may look and feel similar to other problems in which a simpler solution is possible, and I suspected the problem could lead to opportunities for errors and thus for potentially rich discussion. Additionally, playing cards provide a familiar combinatorial context for many students, and I wanted to include some tasks in which the process was more familiar. A similar problem was given in the pilot study, and it yielded rich data.

\subsubsection{3 - The Groups of Students problem}

The Groups of Students problem states, "In how many ways can you split a class of 20 into 4 groups of 5?" A correct answer to this problem is

$$
\frac{\left(\begin{array}{l}
20 \\
5
\end{array}\right) \cdot\left(\begin{array}{l}
15 \\
5
\end{array}\right) \cdot\left(\begin{array}{l}
10 \\
5
\end{array}\right) \cdot\left(\begin{array}{l}
5 \\
5
\end{array}\right)}{4 !}
$$

To arrive at this solution, we choose five students to be in a group, done in $\left(\begin{array}{l}20 \\ 5\end{array}\right)$ ways, then choose 5 of the remaining students to be in another group, $\left(\begin{array}{l}15 \\ 5\end{array}\right)$, then five more to be in a group, $\left(\begin{array}{l}10 \\ 5\end{array}\right)$, and then finally the last five to be in a group, $\left(\begin{array}{l}5 \\ 5\end{array}\right)$. However, after doing this process, we must divide by 4 factorial because the groups are not meant to be 
labeled or distinguished in any way - there is not a Group 1, Group 2, Group 3, and Group 4. The process that led to the numerator

$$
\left(\begin{array}{l}
20 \\
5
\end{array}\right) \cdot\left(\begin{array}{l}
15 \\
5
\end{array}\right) \cdot\left(\begin{array}{l}
10 \\
5
\end{array}\right) \cdot\left(\begin{array}{l}
5 \\
5
\end{array}\right)
$$

being counted as though the groups were distinguishable causes some solutions to show up multiple times, and division by 4 factorial corrects for this. In order to see why we need to divide by 4 factorial, we consider particular outcomes. Suppose the first group that was chosen consisted of kids A, B, C, D, E, (we'll call it Alice's group), the second group, kids F, G, H, I, J (we'll call it Frank's group), the third group, kids K, L, M, N, O (we'll call it Kyle's group), and the fourth group, kids P, Q, R, S, T (we'll call it Paul's group). Then this solution could be written as Alice-Frank-Kyle-Paul, or AFKP, and it denotes the order in which the groups were chosen. However, suppose that each of the groups were the same (Alice's group still consists of A, B, C, D, E, etc.), but that they were chosen in a different order. That is, suppose the solution instead was KAFP or PAKF, representing the same groups that were picked in different orders. The problem only asks for groups of children; there is no first group, second group, etc. Therefore, if the particular division of the class is AFKP, it is the same division as KAFP. In fact, such a division occurs exactly 4 factorial ways (the number of ways of arranging the sequence of letters A, F, K, P). Therefore, division by 4 factorial ensures that each solution gets counted once, as it should be. Another way to think of this is that we may "label" the groups of students (the first group, the second group, etc.) as we use the multiplication 
principle; but, as we do not want the groups to be labeled, we thus divide by 4 factorial to effectively un-label the groups. A typical incorrect solution is

$$
\left(\begin{array}{l}
20 \\
5
\end{array}\right) \cdot\left(\begin{array}{l}
15 \\
5
\end{array}\right) \cdot\left(\begin{array}{l}
10 \\
5
\end{array}\right) \cdot\left(\begin{array}{l}
5 \\
5
\end{array}\right),
$$

where the division by 4 factorial is neglected. The subtlety of the discussion above highlights the fact that this mistake could easily occur, and this possibility for error made it a particularly desirable problem for the study. Note, too, another correct answer is $\frac{20 !}{5 ! 5 ! 5 ! 5 ! 4 !}$. It is equivalent to the correct answer above (which can be checked by application of the formula for binomial coefficients). The solution can be seen by modeling the problem as arranging 20 students in 20 factorial ways (with a group in the first 5 slots, another in the second 5 slots, etc.), and then adjusting for repetition as follows. Each of the 5 factorials in the denominator takes care of the ordering within the 4 groups, and the division by 4 factorial takes care of the ordering of the groups themselves.

This problem was chosen because it fostered the desired situation described above, in which students would be comparing alternative expressions and could potentially have appealed to the set of outcomes to explain any discrepancies. My interview protocol would allow me to facilitate their checking phase of problem solving if they did not do so on their own. Also, unlike some of the other problems, this is a problem that tends to be less familiar to students. I was hopeful that giving students something they were not necessarily familiar with would lead to rich data that could potentially provide further 
insight into their set-oriented thinking. And, too, this is a problem in which the outcomes are not trivial to identify (they are arrangements of 20 students into 4 groups of 5). Such arrangements are abstract to conceptualize, and the large numbers can make conceiving of outcomes particularly difficult. I suspected that it would be worthwhile to see how students would handle a problem in which outcomes were not as concrete as passwords or pairs of cards.

\subsubsection{4 - The Test Questions problem}

The Test Questions problem states, "Suppose an exam consists of 10 questions, and you must answer 5 questions. In how many ways can you answer 5 questions if you must answer at least 2 of the first 5 questions?" This problem again has the at least constraint, and again we provide a solution which involves a case breakdown. We identify sets of five questions in which exactly two, three, four, or five of the first five questions were answered. For any of those cases, the sets of questions containing $k$ questions from the first 5 questions is found by choosing $k$ of those questions (done in $\left(\begin{array}{l}5 \\ k\end{array}\right)$ ways), and then choosing the remaining $5-k$ questions with any of the questions from the second half of the test (done in $\left(\begin{array}{l}5 \\ 5-k\end{array}\right)$ ways). Therefore, the correct result consists of four cases:

$$
\sum_{k=2}^{5}\left(\begin{array}{l}
5 \\
k
\end{array}\right) \cdot\left(\begin{array}{l}
5 \\
5-k
\end{array}\right)=\left(\begin{array}{l}
5 \\
2
\end{array}\right) \cdot\left(\begin{array}{l}
5 \\
3
\end{array}\right)+\left(\begin{array}{l}
5 \\
3
\end{array}\right) \cdot\left(\begin{array}{l}
5 \\
2
\end{array}\right)+\left(\begin{array}{l}
5 \\
4
\end{array}\right) \cdot\left(\begin{array}{l}
5 \\
1
\end{array}\right)+\left(\begin{array}{l}
5 \\
5
\end{array}\right) \cdot\left(\begin{array}{l}
5 \\
0
\end{array}\right)
$$

Another correct approach is to subtract the "bad" cases from the total number of sets of five questions - that is, to subtract the sets of questions that contain fewer than 2 of the 
first 5 questions from all of the 5 -question subsets of test questions. There are $\left(\begin{array}{l}10 \\ 5\end{array}\right)$ total 5-question subsets that could be answered. The total minus bad strategy thus yields the answer

$$
\left(\begin{array}{l}
10 \\
5
\end{array}\right)-\left[\left(\begin{array}{l}
5 \\
0
\end{array}\right) \cdot\left(\begin{array}{l}
5 \\
5
\end{array}\right)+\left(\begin{array}{l}
5 \\
1
\end{array}\right) \cdot\left(\begin{array}{l}
5 \\
4
\end{array}\right)\right]
$$

A tempting solution that does not involve a case breakdown reflects a common error.

We could arrive at an answer of $\left(\begin{array}{l}5 \\ 2\end{array}\right) \cdot\left(\begin{array}{l}8 \\ 3\end{array}\right)$ by arguing that by first choosing two of the first five questions (done in $\left(\begin{array}{l}5 \\ 2\end{array}\right)$ ways), we are guaranteed to have answered "at least two" of the first five questions. We could thus choose any 3 from the remaining 8 questions in $\left(\begin{array}{l}8 \\ 3\end{array}\right)$ ways and still fulfill the constraint. However, the problem with this answer is that some particular outcomes get counted more than once. For example, the set of questions $\{1,2,3,7,8\}$ gets counted multiple times. We could arrive at the answer when questions $\{1,2\}$ were chosen in the $\left(\begin{array}{l}5 \\ 2\end{array}\right)$ step, and questions $\{3,7,8\}$ were chosen in the $\left(\begin{array}{l}8 \\ 3\end{array}\right)$ step. And, this outcome also could have been identified by having first chosen the questions $\{1,3\}$ and then having chosen questions $\{2,7,8\}$.

Like the Passwords problem, the at least constraint in the Test Question problem allowed for me to present students with a seemingly correct answer to the problem that 
was actually incorrect. While the overall structures of these two problems were similar from my perspective (both involved at least constraints), I maintained that this problem differed enough from the Passwords problem still to be interesting. Numerically, the answer to the Test Questions problem is much smaller, and I left the possibility open for some alternative kinds of reasoning or verification than the Passwords problem might have allowed. I also felt that the outcomes of the Test Questions problem (5-question subsets of a 10-question test, in which at least 2 of the first 5 are chosen) are somewhat more abstract in nature than something more concrete like a password. I wanted to give students a problem with the at least constraint that involved a different kind of outcome that they could potentially consider.

\subsubsection{5 - The Apples and Oranges problem}

The Apples and Oranges Problem asks, "How many different nonempty collections can be formed from five (identical) apples and eight (identical) oranges?" To solve this problem, we can choose zero to five apples (six possibilities) and zero to eight oranges (nine possibilities); we cannot choose zero of each, so there are $6 \cdot 9-1=53$ collections. Another common solution to the problem is to consider as cases the different options for the number of pieces of fruit in the collections. There cannot be a collection of size 0 , but there can be collections of size 1 through 13 . These can be counted directly, and the total number of collections is $2+3+4+5+6+6+6+6+5+4+3+2+1=53$.

In Section 2.2, I described a solution to this problem by Holly, a pilot study participant; we saw in that excerpt how set-oriented thinking arose as Holly worked 
through this problem. In the pilot study, this task yielded a variety of interesting solutions, and the reason for the inclusion of this task was thus the potential for rich mathematical discussion. Another desirable feature of this problem is the fact that in my experience with it as a teacher and a student, for many students it is not an easilyidentifiable problem type. I hoped that including it could allow students to reason about the problem in new ways and not rely too heavily on familiar formulas or problem types. And, too, the numbers were small enough to encourage some experimentation if a student was unsure of how to proceed.

\section{5 - Data analysis}

Having described the subject selection, data collection, interview protocol, and task selection, I now describe the analysis of the data. I begin by describing the principles of analysis that drove my study. I then describe what I did as I analyzed Stage 1, and I will discuss the effects that it had on my interviews in Stage 2. Then, I give a detailed account of the overall analysis I conducted on the entire set of Stage 1 and Stage 2 data combined.

\subsection{1 - An analytical framework: Grounded theory}

I utilized the methodological perspective of grounded theory as I analyzed my data, particularly Auerbach and Silverstein's (2003) steps for developing theory from raw text and Strauss and Corbin's (1998) detailed coding processes. Given the relatively new field of combinatorics education, my research, including any hypotheses or ideas that are being formed, is still at a very preliminary stage. Because of this, I am still in the phase of 
forming and articulating conjectures and hypotheses, and grounded theory is well-suited as a methodological framework for this study.

In their book on qualitative research, Auerbach and Silverstein (2003) offer an insightful description of grounded theory, as do Strauss and Corbin (1998). While I use Auerbach and Silverstein's language in much of what follows, I will at times refer to Strauss and Corbin as well. For my purposes, grounded theory is useful as a methodological guideline. In this section, I discuss some of the main ideas behind grounded theory, with the understanding that specific methodological concerns will be addressed in the Methodology chapter.

Grounded theory was originally developed in the 1960's and 1970's by two sociologists, Glaser and Strauss. As they conducted qualitative analysis, they recognized the need for "making comparisons between data to identify, develop, and relate concepts" (Strauss \& Corbin, 1998, p. 10). What has emerged is an alternative to the traditional, quantitative, hypothesis-testing research methodologies that dominated research for many years. At the core of grounded theory is the premise that, at times, researchers may wish to study phenomena about which no previously-existing theory or construct exists. That is, they may find themselves in a situation where they need to develop theory, rather than to implement or test theory. Grounded theory is a research methodology created to support such research needs.

The basic idea behind grounded theory stands in contrast with standard quantitative methods. Rather than using data to test already-established hypothesis, grounded theory seeks to develop hypotheses, allowing the data to influence directly the formation of 
conjectures and, eventually, theoretical constructs. This theory has been subsequently used, described, and developed by other theorists as well (e.g., Charmaz, 2006; Strauss \& Corbin, 1998). I now outline some of the main ideas of qualitative research and grounded theory, as put forth by Auerbach and Silverstein.

3.5.1.1 - Designing a study using grounded theory. Auerbach and Silverstein (2003) begin by articulating a distinction between quantitative and qualitative studies; they label them as hypothesis-testing and hypothesis-generating studies, respectively ${ }^{10}$. They list the following five steps as typical of the design of a quantitative study which seeks to test a hypothesis: 1) Conduct a literature review and identify a research problem; 2) Develop a research hypothesis; 3) Operationalize the variables; 4) Establish a random sampling technique; and, 5) Determine sample size. According to Auerbach and Silverstein, following these steps, and conducting appropriate statistical analysis of the data, would result in a study that allows for the testing of a particular hypothesis. In contrast to these stages, they describe five steps that are characteristic of a qualitative design that utilizes grounded theory.

Step 1: Research issues (instead of research problems). Auerbach and Silverstein argue that under grounded theory, it may not be possible to glean a specific research problem from the literature; indeed, something much more vague, or something that is completely absent from the literature, might emerge from a literature review. They

\footnotetext{
${ }^{10}$ It could be argued that this is an oversimplification of the relationship between qualitative and quantitative research; one can generate hypothesis from quantitative work and test them with qualitative methods. I have presented this notion as Auerbach and Silverstein (2003) proposed it.
} 
therefore use the term research issues, which they take to be more general than research problems, to allow for such a possibility.

Step 2: Research concerns (instead of research hypotheses). According to Auerbach and Silverstein, "because grounded theory does not assume that the researcher knows enough to formulate specific hypotheses," (p. 15), the progression is from research issues to research concerns, not to specific research hypotheses. They use the broader term research concerns, and intentionally do not use the term hypotheses, to articulate this distinction. According to them, a study is not designed with specific hypotheses in mind; rather, hypotheses will emerge as a product of the research.

Step 3: Narrative interviewing (instead of operationalizing variables). In this stage, the researcher engages with participants with the intent of gathering information, and with the mindset that the participants may bring up unanticipated topics. Indeed, these interviews are marked by flexibility by the researcher, who is open to the emergence of new ideas.

Step 4: Theoretical sampling (instead of random sampling). Auerbach and Silverstein note that "Grounded theory research does not think that random sampling is realistically possible, and approaches generalizability in terms of developing hypotheses, not testing them" (p. 17, italics in original). To them, randomization of a sample is often not possible, not to mention not particularly helpful in the grounded theory framework. Rather, theoretical sampling allows the researcher to select participants based on their potential to help get at the research concerns. They note that "In this way, your theory, 
rather than the requirement of randomness, determines which research participants will constitute your sample” (p. 19).

Step 5: Theoretical saturation (instead of statistical significance). Auerbach and Silverstein note that in quantitative studies, the goal is to measure the usefulness of the results by appealing to some sort of statistical significance. However, this kind of measure has less meaning in qualitative studies in which hypotheses are not being tested and samples sizes are relatively small. They describe an important construct of theoretical saturation as being critical in determining sample sizes in such a qualitative study. In particular, they note that because grounded theory research is more concerned with the construction and generation of theory (as opposed to the testing of theory), each group of research participants interviewed embodies a new opportunity to develop and refine theory. Therefore, in grounded theory research one continues interviewing research participants until they find that new groups of participants are not producing new data that add new concepts to the theory (p. 19). They call this procedure theoretical saturation. Thus, theoretical sampling and theoretical saturation together help to determine the sample size.

These steps above are written by Auerbach and Silverstein (2003) as a sort of "recipe" (p. 20) for designing a qualitative study. They define qualitative research as "research that involves analyzing and interpreting texts and interviews in order to discover meaningful patterns descriptive of a particular phenomenon" (p. 3). The intent of the preceding discussion is to denote specific differences between quantitative and 
qualitative studies, and to highlight the variety of research methodologies that could be associated with each.

3.5.1.2 - Grounded theory and analysis. Beyond addressing aspects of the design of the study, grounded theory is inextricably linked with the data analysis process. That is, because the kinds of data, as well as the kinds of products of the research, are quite different between qualitative and quantitative studies, Auerbach and Silverstein (2003) (along with other grounded theorists such as Strauss and Corbin (1998)) offer specific details about the details of the analysis and coding processes. Data analysis, and the coding of data, is arguably the most significant aspect of grounded theory. I offer a broad outline of Auerbach and Silverstein's coding scheme here, but I will discuss it in more detail in the Methodology chapter. I will also describe particular coding that Strauss and Corbin advocate.

Again, the main point of grounded theory is to try to glean patterns and themes (and ultimately theory) by open-mindedly reviewing the data - the data itself is integral in the development of themes. Auerbach and Silverstein (2003) suggest specific ways in which coding ought to take place, noting that their coding method is "based on the premise that no one is smart enough or intuitive enough to read a series of transcripts and immediately see the patterns within them" (p. 31). The coding method allows the researcher to begin with a massive amount of raw data, to organize and make sense of this data by noting recurring patterns and themes, and then ultimately to develop theory from the patterns. Researchers using grounded theory find themselves in the midst of massive amounts of raw data in the form of transcripts of videotaped interviews, and they must organize and 
make sense of that data in order to emerge with hypotheses and constructs related to their research concerns. But how does one get there? Auerbach and Silverstein offer the following hierarchical sequence to progress from raw text to research concerns: raw text, relevant text, repeating ideas, themes, theoretical constructs, theoretical narrative, and research concerns. I now briefly describe each of the stages of the progression.

To Auerbach and Silverstein (2003), the raw text consists of the transcripts of videotaped interviews. From here, the researcher can cut down the text to a more manageable quantity by reading through the raw text with the overall research goals in mind. That is, the researcher can read through the raw text and highlight the relevant parts of the text that pertain to the research in which he is interested; this process makes the text easier to work with. Then, the researcher can look back through the relevant text, looking for repeating ideas that shed light on the research concerns. Then, as repeating ideas are noted, groups of repeating ideas that have something in common should begin to emerge - Auerbach and Silverstein call what they have in common a theme, "an implicit topic that organizes a group of repeating ideas" (p. 38). These themes are then organized into larger, abstract ideas. Auerbach and Silverstein refer to the abstract grouping of themes as theoretical constructs. These theoretical constructs can then be organized into a theoretical narrative, "which summarize(s) what we have learned about our research concerns" (p. 40). That is, the narrative, which is based on theoretical constructs, which were based on themes that stemmed from repeating ideas that emerged from the data, can give a sense of how the research concerns are related to the raw data. This narrative "bridges the gap between research concerns and raw data" (p. 41). 
Strauss and Corbin (1998) offer further details about types of coding in grounded theory; I present them now and will specify how they arose in my study when I discuss my analysis in the Methodology chapter. Strauss and Corbin make a distinction between three kinds of coding: open coding, axial coding, and selective coding. Each of these plays a role in the development of theory from raw data, and I briefly outline each of these in the paragraphs that follow.

Open coding. Strauss and Corbin (1998) define open coding as "the analytic process through which concepts are identified and their properties and dimensions are discovered in data" (p. 101). The focus of open coding is on concepts, which the authors define as labeled phenomena (p. 103), and open coding is so named because "to uncover, name, and develop concepts, we must open up the text and expose the thoughts, ideas, and meanings contained therein" (p. 103). In open coding, a researcher will go through the data, carefully identify phenomena, and name them. Strauss and Corbin note that open coding typically consists of line-by-line analysis, in which each word is carefully analyzed, but they note that analyzing whole sentences or paragraphs could also be a way in which to implement open coding.

Axial coding. Once those labeled phenomena are identified, researchers can then engage in axial coding ${ }^{11}$. Strauss and Corbin (1998) note that axial coding allows the researcher "to begin the process of reassembling data that were fractured during open

\footnotetext{
${ }^{11}$ This type of coding is called axial because coding occurs around the axis of a category, linking categories at the level of properties and dimensions (p. 123).
} 
coding" (p. 124). In particular, the product of a researcher's open coding may be some number of labeled phenomena. In axial coding, the task is now to consider how those phenomena relate to each other. Strauss and Corbin characterize axial coders as helping researchers "relate categories to their subcategories" (p. 124) and "contextualize a phenomenon" (p. 127). This kind of coding does not necessarily involve the line-by-line analysis of open coding; instead the coders sort through the concepts they have identified and try to articulate ways to organize them in relation to each other.

Selective coding. Selective coding is defined as "the process of integrating and refining theory" (p. 143). In this type of coding, the researcher now formulates theory by describing connections and relationships between the categories that came out of their axial coding. This involves more than simply identifying a number of interesting phenomena or ideas that came out of the data; it involves careful thought as to the structure of such themes. As Strauss and Corbin (1998) describe, "if theory building is indeed the goal of the research project, then findings should be presented as a set of interrelated concepts, not just a listing of themes" (p. 145). The development of theory that occurs through selective coding is thus rooted in both open and axial coding, and it requires coordination and structuring of major themes that have emerged from the data.

Thus, Auerbach and Silverstein (2003) and Strauss and Corbin (1998) have outlined a systematic process that will enable someone to maneuver through the data in its very rawest form and to emerge with a meaningful narrative that relates directly to the research concerns. As mentioned above, further details of this coding process will be discussed in the Methodology chapter in conjunction with other methodological 
concerns. However, this brief discussion of grounded theory, as well as the introduction of some of Auerbach and Silverstein's and Strauss and Corbin's particular language, is intended to provide the reader with a sense of the theory that has grounded and shaped the design of the study. This study is one in which I hope to form, and not test, hypotheses, in which I seek to make connections and open-mindedly learn what conjectures, issues, and questions will emerge from the data. Therefore, I have drawn upon grounded theory for specific ways to design my proposed qualitative study. While Auerbach and Silverstein (2003) served as a valuable resource for the larger picture of where I was going (raw text to research concerns), I also drew upon Strauss and Corbin's (1998) discussion of grounded theory in some key steps of my analysis. In particular, I used their notions of open coding, axial coding, and selective coding as I moved from the videotape data and transcripts to the theory that has emerged in my Conclusions chapter. I have outlined these types of coding in the Literature Review chapter, and I will address how they arose specifically as I explicate my analysis in the sections to follow.

While grounded theory is the key methodological perspective on which I drew, I also used Powell et al.'s (2003) seven-phase analytical model for studying the development of mathematical thinking (particularly from videotape data). Powell et al. (2003) suggest the following sequence of seven "interacting, non-linear phases" (p. 413) as the basis for their analytical process:

1. Viewing attentively the video data

2. Describing the video data

3. Identifying critical events

4. Transcribing

5. Coding 
6. Constructing storyline

7. Composing narrative

Auerbach and Silverstein's (2003) steps in grounded theory (p. 35) are those listed below; I number them for future reference.

1. Raw Text

2. Relevant Text

3. Repeating Ideas

4. Themes

5. Theoretical Constructs

6. Theoretical Narrative

7. Research Concerns

As can be seen above, both 7-step processes above, the models are similar in that they describe methods of building up a story from data. However, there are differences as well. The analytical process suggested by Powell et al. (2003) does not start from the raw text of the transcripts. Rather, Powell et al. identify and describe methods by which to get from the videotape data to the transcripts. These authors seem to value the videotapes highly as data (that is, they do not view the video data and the transcripts as being the exact same set of data), and thus they spend considerable time examining the video data before they create the transcripts. In contrast, Auerbach and Silverstein's (2003) coding process begins once the transcripts (the raw text) have already been made; they provide little comment on how to get from the videotape data to the raw text. In light of this, it seems that all of Auerbach and Silverstein's seven steps actually fit into steps 5-7 of Powell et al. I attempted to weave both of these ideas into one analytical process, drawing upon Powell et al. for analysis before and up to transcription, and drawing upon Auerbach and Silverstein and Strauss and Corbin for specific coding once transcription 
was completed. The process described by Powell et al. were particularly important during the Stage 1 interviews, in which it was important for me to conduct some analysis between two interviews with the same student, but for which entire transcriptions of interviews was not possible in the time allotted. These two analytical influences complement each other, each providing detail where the other framework is lacking it. Thus, together these two frameworks formed a complementary analytical system for my study.

I interviewed each student twice in the first stage of interviews and each student once in the second stage. I will briefly describe the details of the analysis of Stage 1 below, and I will briefly mention analysis of the Stage 2 data, but the majority of the analysis (and indeed the findings in my results chapter and the conclusions I have drawn) was done on both Stages of data as a whole. Therefore much of what I discuss in the rest of the chapter relates to my reflective analysis of the entire data set (all 22 students' work on the five problems discussed above).

As discussed in my theoretical frameworks chapter, on a broad level I wanted to utilize Auerbach and Silverstein's (2003) plan for analyzing data using grounded theory. And, indeed, globally, this is what I did. Beginning with video data, and then the transcripts, through much coding and evaluation and thought I eventually emerged with theory. I also drew upon Strauss and Corbin's (1998) particular stages of coding in order to realize this development of theory from raw data. I began with video data and transcripts and have ultimately ended up with a theoretical contribution related to students' set-oriented thinking as they solve counting problems. 
As I describe the analysis to follow, I want to give some sense of the progression as my analysis developed. Thus, while some of what follows is not what I ultimately found to be most useful (and involves mathematics that I did not emphasize in the end) it is an accurate depiction of my analysis process. Particularly given the grounded theory on which my analysis is based, it is important to see some specifics of how the final conclusions emerged from the raw data. This section will thus serve to elaborate the process that took me from my raw videotape data to the development of the theory that is described in my conclusions.

\subsection{2 - Data collection and analysis in Stage 1}

3.5.2.1 - Collection and organization of Stage 1 data. I interviewed 8 students in Stage 1, and for each of these I both conducted the interview and ran the camera. Following each interview I wrote a reflection on my general impressions, giving a description of what happened and also making note of any particularly relevant episodes. The purpose of this reflection was to try to retain any thoughts, ideas, insights, and impressions from that interview, and to help me plan for the second interview with the same student. In Stage 1, I interviewed each student twice, and the length of time between the two interviews ranged from one day to two weeks, depending on the schedules of the particular students. Between a student's pair of interviews I reviewed what had happened in their first interview and made a plan for what I would try to cover in the second interview. Because I thought that the evaluation of alternative expressions might have required a substantial amount of time in some cases, I did not expect to cover all of the 
problems in the second interviews. During the analysis of the first interview I thus determined and prioritized which problems I would cover in each second interview, and I prepared alternative answers based on what each student had done initially.

To prepare for second interviews, I viewed the videotape of each first interview and made a content $\log ^{12}$. More specifically, producing content logs involved re-watching the interviews and taking notes on what had transpired. The logs consisted of three columns; in the first I marked the time at which a new problem (or a particularly noteworthy episode) began, in the second I wrote a narrative description of what was happening during the interview, and in the third I wrote down my own thoughts and interpretations of what was happening during the episodes. The content logs allowed me to take another pass through the data, helped me to gain a sense of the overall narrative of the interview, and enabled me to flag any potentially interesting episodes (see Appendix $\mathrm{C}$ for an example of a content $\log$ ). This process of viewing the data and writing a content log utilized the first three stages of Powell et al. (2003)'s framework - viewing attentively the video data, describing the video data, identifying critical events.

After I finished conducting all of my interviews, and after I wrote the content logs, I transcribed both interviews for each of the 8 students, totaling 16 interviews for Stage 1. This was a valuable experience and an indispensable aspect of my overall analysis. Both Powell et al. (2003) and Auerbach and Silverstein (2003) are proponents of the

\footnotetext{
${ }^{12}$ I also wrote content logs after the second interviews, and these content logs gave me another pass through the data, allowing me to further familiarize myself with the students' work on the problems.
} 
transcription of videotape data. Powell et al. acknowledge the importance of transcription in a study which, like mine, is guided by grounded theory. They note that, "the production of the transcript and the physical, static rendering of a research session afford researchers opportunities for extended, considered deliberations of talk and noted gestures (p. 422)." The transcription facilitated a deeper level of analysis, allowed me become extremely familiar with students' utterances, strategies, successes and failures on each problem.

After I completed the transcripts from the Stage 1 interviews, I organized the data using Microsoft Excel. I made one workbook for each of the 8 students, and in that workbook each problem had its own spreadsheet (I have included a screenshot of Kristin's Excel file in Figure 2 below; this was her work on the Test Questions problem, and the black line separated her work on the problem initially from her work on the problem as she evaluated alternative answers). 


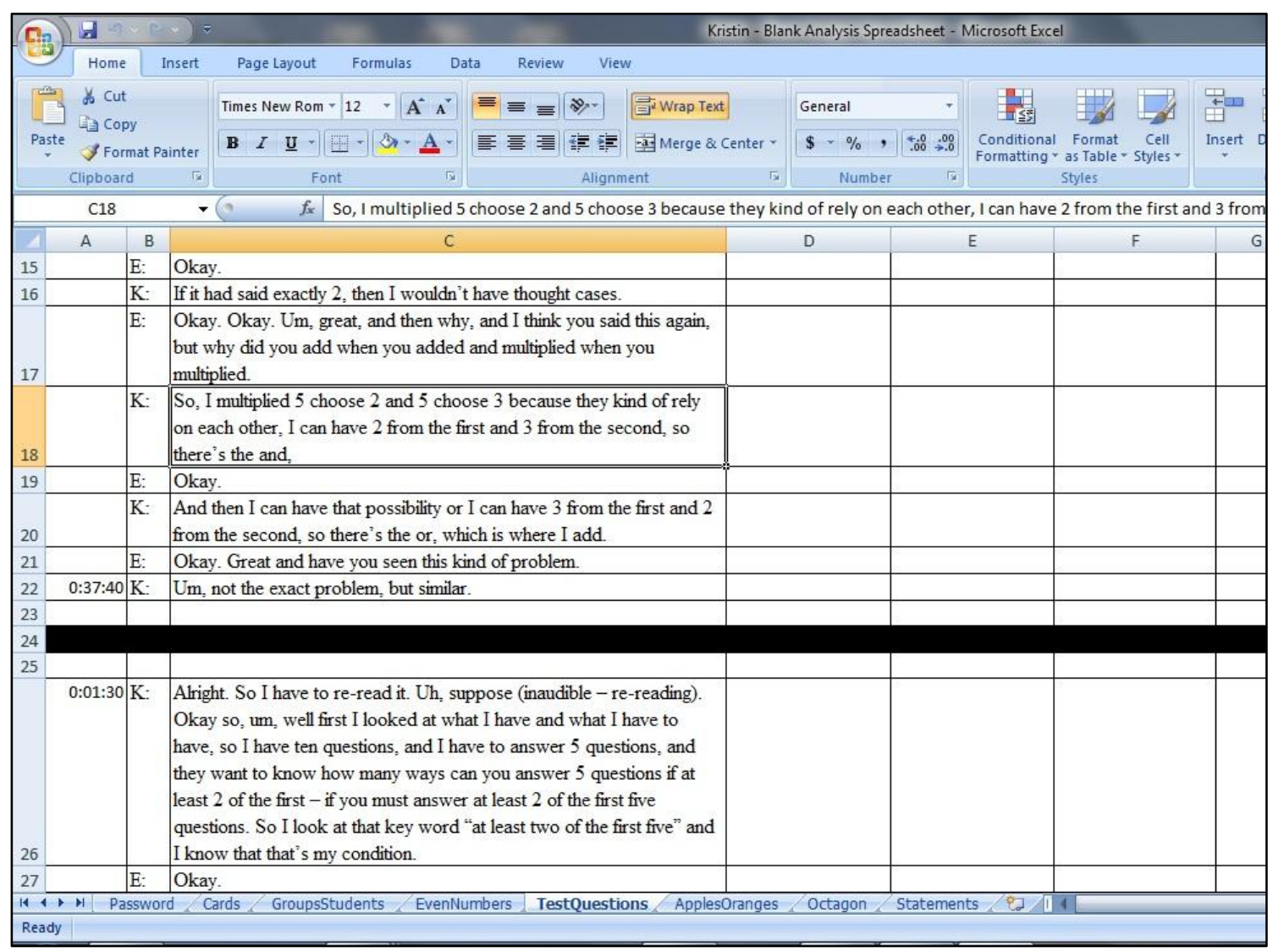

Figure 2

Since the interviews switched back and forth between the student and me talking, each row of the spreadsheet consisted of an entire turn by either the student or me. Thus the numbered rows alternated between the student's dialogue and my own. This format gave me easy access to any students' work on a particular problem. Another positive feature of this organization was that I could easily make notes in columns to the right of the column that contained the transcription. Additionally, I could color-code the transcript and generally manipulate the text for my analytical needs. In subsequent examples of coding I will highlight some of the ways I utilized these features of the spreadsheet. 
3.5.2.2 - Analysis of Stage 1 data. After I had settled on this organization of the data, I began to analyze it, and I particularly looked to code the data so that interesting phenomena (which I could eventually formulate into themes and hypotheses) would emerge. Before I proceed with descriptions and examples of my analysis, I first make a brief interjection about language. Because my analysis is based on those observable elements of students' activity, language is a primary source from which to determine phenomena. In this section, and in sections to follow, I will at times use the phrase "settheoretic language." I take set-theoretic language to be that language which explicitly refers to principles of set theory - those terms that might be in a glossary of a set-theory textbook and that have accepted set-theoretic meaning in the mathematical community. Specifically, I consider the following such words to be set-theoretic: set, element, subset, union, intersection, complement, Cartesian product, partition, cardinality, and disjoint. This is not an exhaustive list of such terms, but these words have meaning in the context of set theory, and this gives a sense of what I mean by "set-theoretic." Students may use language which implies they consider set theory or the set of outcomes; they may, for example talk about "dividing up a group into subgroups that do not overlap" instead of using the word "partition," but I take set-theoretic language to be language in which they clearly use accepted set-theoretic terminology. In Section 3.5.4 I will language (settheoretic and not) that I took as evidence that a student considered a set of outcomes.

In my analysis of the Stage 1 data, my goal was to ultimately be in a place where I could formulate some hypotheses which could be tested and either substantiated or disconfirmed in Stage 2. While I knew that Stage 2 would bring new data that might 
cause me to change my hypotheses (or create new hypotheses), I still wanted to define some hypotheses at the end of my analysis of Stage 1 and before I began the Stage 2 data collection.

In an attempt to form such hypotheses, I then engaged in what Strauss and Corbin (1998) call open coding, which they define as, "the analytic process through which concepts are identified and their properties and dimensions are discovered in data" ( $\mathrm{p}$. 101). Specifically, I began by going through the transcripts line by line, identifying particular phenomena that seemed interesting and relevant. Having interviewed the students, written content logs, and transcribed their interviews, I already had some idea of some of the phenomena that seemed particularly noteworthy. I began to label the phenomena I observed, which is a key element of open coding. At the time I was very focused on both set and process-oriented thinking, and so a primary phenomenon I paid attention to was whether a particular utterance was set-oriented or process-oriented. Four of my main codes were thus "Implicit Set," "Implicit Process," "Explicit Set," and “Explicit Process," as described below, but I noticed other phenomena as well, especially language and episodes that came up frequently, such as students" use of "slots" and “choices" language, and students' tendencies to use tree diagrams. What emerged from some hours spent performing line-by-line analysis of the transcript was a preliminary list of codes that represented what I considered to be relevant phenomena that I had observed in these interviews. I provide an example of the format of the list of codes in Table 3 below. 


\begin{tabular}{|c|c|c|}
\hline Brief Description & Code & Detailed Description and Example \\
\hline $\begin{array}{l}\text { Students explicitly talk about } \\
\text { sets }\end{array}$ & $\begin{array}{l}\text { EXPSET } \\
\text { (Explicit Set) }\end{array}$ & $\begin{array}{l}\text { Students are talking in terms of sets, with explicit, set- } \\
\text { theoretic language. } \\
\text { Example: Daniel, Int 2, 0:27:27, "And, kind of like I } \\
\text { was saying earlier, I mean, any, any way of answering } \\
\text { any number of questions on the quiz is like a subset of, } \\
\text { you know, the set where you have, like, all of the } \\
\text { possible answers, right?" }\end{array}$ \\
\hline $\begin{array}{l}\text { Students explicitly talk about } \\
\text { processes }\end{array}$ & $\begin{array}{l}\text { EXPPRO } \\
\text { (Explicit } \\
\text { Process) }\end{array}$ & $\begin{array}{l}\text { Students are talking in terms of process, with explicit, } \\
\text { process-oriented language. }\end{array}$ \\
\hline $\begin{array}{l}\text { Students' language indicates } \\
\text { set-theoretic reasoning, but } \\
\text { they do not explicitly talk } \\
\text { about sets }\end{array}$ & $\begin{array}{l}\text { IMPSET } \\
\text { (Implicit Set) }\end{array}$ & $\begin{array}{l}\text { There is some appeal to a set of objects that is being } \\
\text { counted. } \\
\text { Example: Nancy, Int 2, 0:33:15, "And that's going to } \\
\text { be counted twice I think." }\end{array}$ \\
\hline $\begin{array}{l}\text { Student's language indicates } \\
\text { process-oriented reasoning, } \\
\text { but they do not explicitly talk } \\
\text { about processes }\end{array}$ & $\begin{array}{l}\text { IMPPRO } \\
\text { (Implicit } \\
\text { Process) }\end{array}$ & $\begin{array}{l}\text { The language seems to indicate a process way of } \\
\text { thinking, in which students describe stages or steps in } \\
\text { a procedure. } \\
\text { Example: Interview 2, 0:51:08, "If you select your } \\
\text { three E's, then, uh, you could select anything else, and, } \\
\text { uh, I mean, you choose } 3 \text { E's out of the } 8 \text { characters, } \\
\text { then, uh, it doesn't matter what else you choose, there } \\
\text { will always be, um, E's." }\end{array}$ \\
\hline $\begin{array}{l}\text { Single statements/excerpts } \\
\text { where the student seems to } \\
\text { be simultaneously using both } \\
\text { set and process thinking }\end{array}$ & $\begin{array}{l}\text { BOTH } \\
\text { (Both sets and } \\
\text { processes). }\end{array}$ & $\begin{array}{l}\text { This seemed to happen a lot for Daniel - possible } \\
\text { because I think he had a really strong sense of each } \\
\text { kind of thinking. Within these statements there seem to } \\
\text { be literally process aspects and set aspects. } \\
\text { Example: Second interview, } 0: 32: 00 \text {, "But it doesn't } \\
\text { show - unfortunately it shows a way to count some of } \\
\text { the answers more than once." } \\
\text { Example: Second interview, } 0: 36: 11 \text {, "Those two ways } \\
\text { of choosing are actually choosing the same, uh, } \\
\text { combination of answered questions." }\end{array}$ \\
\hline
\end{tabular}

Table 3

Figure 3 below provides an example of some of this initial coding. It is an excerpt taken from Casey's work on the Passwords problem, I coded the utterances using the list of 
codes I had established. I had developed a color-coding scheme in which set-related utterances were coded as blue and process-related utterances were coded as red, and anything that might have been some combination of the two was coded as purple. At the time I felt that these colors would enable me to get a sense of when set or processoriented thinking occurred in the interview.

\begin{tabular}{|c|c|c|c|c|}
\hline 65 & $0: 51: 08$ & E: & $\begin{array}{l}\text { Okay so can you just talk about, well talk initially maybe what you had } \\
\text { thought initially about the } 8 \text { choose } 3 \text { times } 26^{\wedge} 5 \text {. }\end{array}$ & \\
\hline 66 & & D: & $\begin{array}{l}\text { Okay, um, I just thought, uh, if you select your three E's, then uh, you } \\
\text { could select anything else, and, uh, I mean, you choose } 3 \text { E's out of the } 8 \\
\text { characters, then, uh, it doesn't matter what else you choose, there will } \\
\text { always be, um, E's. But the problem is that if you have E's in those other } \\
\text { characters that you choose, then, uh, then that's going to count some } \\
\text { things twice, so. }\end{array}$ & BOTH, OBJECT \\
\hline 67 & $0: 52: 07$ & E: & Okay, then talk me through what this one is doing. & \\
\hline 68 & & D: & $\begin{array}{l}\text { So this one is, uh, basically the same as I was doing in the other case. } \\
\text { You partition the combinations by the, uh, count of, um, by the count of } \\
\text { E's, and then, um, because each solution, yeah, then each solution, or } \\
\text { what do I want to say, each of these ways of counting only counts the } \\
\text { thing in one partition - or what, in one set, or in one element of the }\end{array}$ & EXPSET \\
\hline 69 & & E: & Sure, okay. & \\
\hline 70 & & D: & So, uh, there's no overlaps between them. & OVERLAP, EXPSET \\
\hline 71 & & E: & Okay. & \\
\hline 72 & & D: & $\begin{array}{l}\text { So if you count all the cases, and vou count evervthing in each case, then } \\
\text { you don't have any overlaps and then you don't have anything missing. } \\
\text { Dang (or I think). }\end{array}$ & CASE \\
\hline
\end{tabular}

Figure 3

In addition to the coding I had done thus far, I had also wanted to keep in mind Carlson and Bloom's (2005) problem solving phases as a lens through which to analyze my data ${ }^{13}$. During my analysis of Stage 1 , then, I paid attention to the emergence of

\footnotetext{
${ }^{13}$ Carlson and Bloom (2005) put forth a problem solving framework which includes, among other elements, a four-stage problem solving cycle which included orienting, planning, executing, and checking phases. In my analysis of Stage $1 \mathrm{I}$ had, although ultimately the problem solving phases did not particularly have an impact on my overall analysis, my results, or my conclusions.
} 
problem solving phases and tried to keep track of any relationships between the phenomena I was finding and the problem solving phases. To this point, this analysis had remained relatively informal - I had done some color coordinating involving the problem solving phases and had guessed at some relationships between problem solving phases and instances of set-oriented thinking (the purple shading in Figure 3 above indicates an early attempt at coding for the checking phase).

The list of codes above facilitated further engagement with the data and allowed me to develop preliminary hypotheses so I could proceed with Stage 2 interviews, and a couple of issues came up for me after my coding of the Stage 1 data that had an effect on the design of my study. I mention these now, as I feel this discussion explains some of the shift in my perspective from a distinction between set-oriented and process-oriented thinking to an emphasis on the ways in which students use set-oriented thinking.

The open coding that I had done to this point conferred two major benefits. First, it yielded a list of relevant phenomena that I had observed as students solved counting problems. This list was a first attempt at identifying key concepts in my data, and while it felt unwieldy at times, it was nonetheless beginning to give some structure to what I was finding in my data. Second, the process of conducting the line-by-line open coding made me keenly aware that a shift in my research concerns was needed. In particular, when I initially began my open coding, I was quite interested in investigating the distinction between set and processes-oriented thinking. I thus began to code expecting to label utterances as involving sets or involving processes. When I actually began to code the data, though, I often found single utterances that warranted codes involving both 
processes and sets, and I therefore became more interested in the connection among these ways of thinking and less interested in the distinctions between them. To be more specific, my analysis was making me more aware of the fact that there was not a clear dichotomy between set-oriented and process-oriented thinking, and that it might not be possible to articulate a distinction easily. As an example of some lines that I felt involved both set and process-oriented thinking, we see Casey's work on the Test Questions problem (Figure 4).

\begin{tabular}{|c|c|c|}
\hline 171 & Hmm. Ahh. & \\
\hline $172 \underline{\underline{F}}$ & Okay what. & \\
\hline 173 & $\begin{array}{l}\text { Hang on, alright, so I've had a thought, okay. So, uh, uh, it occurs } \\
\text { to me that my solution is counting too many, uh, iust from the first } \\
\text { 3, uh, sets or possibilities that I have here, it's, just enumerating - } \\
\text { answering the first four and then, uh, one from the remaining } 6 \text {, }\end{array}$ & BOTH? \\
\hline $174 \overline{\mathrm{B}}$ & Okay. & \\
\hline 175 & $\begin{array}{l}\text { Uh, or questions } 2 \text { through } 5 \text { and then one left over, and then } 1 \text {, } \\
345 \text { and then } 1 \text { left over. Um, then I realized that you can answer, } \\
\text { you know, the one before what you've already answered, or, one in } \\
\text { the middle of what you've already answered. But in that case this - } \\
\text { oops, is exactly the same as this. }\end{array}$ & \\
\hline $176 \overline{\mathrm{B}}$ & Okay. & \\
\hline $\begin{array}{r} \\
177 \\
177\end{array}$ & $\begin{array}{l}\text { So now, and this is } 6 \text {, there's } 6 \text { here, } 6 \text { here, and } 6 \text { here, and there } \\
\text { are going to be, and I assume that it's going to be } 5 \text {, uh, different } \\
\text { ways of, uh, ordering the first four question, it will be } 6 \text { times } 5 \text { is } \\
30 \text {, which is to arrive at my answer, but then there are going to be } \\
\text { too many of them because answering the first five in a row is } \\
\text { counted, uh, too many times, } 5 \text { too many times in fact, which gets } \\
\text { us to } 25 \text {, so there you go. }\end{array}$ & BOTH OR IMPSET? \\
\hline
\end{tabular}

Figure 4

In line 173 I was unsure of whether or not Casey's language suggested set-oriented thinking or process-oriented thinking, and I felt like there were aspects of both. He 
certainly seems to articulate outcomes, and in fact he uses the term "sets of possibilities" which suggests to me an ability to consider the set of outcomes he is counting. However, his statement, "my solution is counting too many" is noteworthy, and to me it suggested that Casey was considering some kind of process (the process reflected in his solution) that was "counting too many." When my paradigm had been focused on a distinction between the two kinds of thinking, I felt unsure of how to code such an utterance, and I thus coded it as "BOTH." As I continued to code there were increasing instances that I felt could be assigned a "BOTH" code, and my notion of these ways of thinking and their interaction began to change. As a result of this, I began to change my focus away from an emphasis on some dichotomy between set-oriented and process-oriented thinking (I was not sure such a dichotomy existed), and instead concentrate on identifying ways in which students utilized set-oriented thinking as they counted. It was thus through my initial experience with open coding that I realized an adjustment might be warranted.

3.5.2.3 - Hypotheses from Stage 1. Bearing in mind the changes that I was considering in light of my open coding of the Stage 1 data, I proceeded with some axial coding of the Stage 1 data in order to articulate some hypotheses from Stage 1. Specifically, I framed some of those phenomena that I had labeled in Stage 1 into hypotheses with which I could begin the second stage of my study. I now discuss the hypotheses that emerged after my initial coding and after a realization that I wanted to pay particular attention to 
ways in which students used set-oriented thinking in their counting ${ }^{14}$. At the time the hypotheses were developed, they were only loosely organized, and they were in some ways still quite preliminary, but they indicate some sense of direction as I moved forward with Stage 2. I will briefly discuss each hypothesis after I state it.

- Hypothesis 1: Students utilize set-oriented thinking for their benefit as they solve counting problems.

- Hypothesis 1a) Students utilize strategies such as case breakdowns and totalminus-bad in order to organize their work and determine appropriate courses of action. These strategies can indicate a consideration of a set of outcomes.

- Hypothesis 1b) As they evaluate alternative solutions, students use setoriented thinking in order to discover and identify errors of over-counting, particularly through the identification of a particular element of the set of outcomes that was counted more than once.

- Hypothesis 1c) When students are stuck in their work on a counting problem, set-oriented thinking can be a resource to give them an alternate solution strategy and help them progress in their problem solving.

\footnotetext{
${ }^{14}$ I had created a couple of different versions of these hypotheses, one presented as an outline with supporting examples, and one expressed more in terms of the underlying set theory I felt was behind them. The list presented here is representative of the hypotheses as they were presented in both of those versions.
} 
- Hypothesis 1d) Set-oriented thinking may provide students with elements of verification strategies, which tend to be elusive in counting problems (Eizenberg \& Zaslavsky).

Hypothesis 2: There are other ways (not found in Stage 1) in which set-oriented thinking could arise, that might become apparent in students with greater facility with set theory. This second hypothesis stemmed from the fact that set-oriented thinking had not come up very often in Stage 1, and I wanted to keep open the possibility that more set-oriented thinking could arise for students. As I have explained, the ideas that led to this hypothesis contributed to decisions about subject selection for Stage 2 .

\subsubsection{4 - Changes to Stage 2 based on Stage 1 interviews}

As a result of my analysis of Stage 1, there were three practical changes that I made as I prepared for Stage 2. First, I realized that because I was planning on interviewing more students in Stage 2 than I had in Stage 1 (12-15), I looked for ways in which I could efficiently streamline the interview process. Practically, this meant that I decided to limit the problems from 7 to 5 , and the choice of which problems to cut was based on my experience in Stage 1. I found upon analysis that both the Even Numbers problem and the Octagon problem ${ }^{15}$ did not seem to yield as much rich data as some of the other problems did. The Octagon problem, which was the last problem I posed in the interview, had

\footnotetext{
${ }^{15}$ The Even Numbers problem states, "How many even, 5-digit numbers (no leading zeros allowed) are there with distinct digits?" The Octagon problem states, "How many triangles can be formed by joining different sets of three corners of an octagon? How many triangles if no pair of adjacent corners is permitted?"
} 
occasionally been skipped in the first interviews in Stage 1 and had not been revisited often, and neither problem had the same kind of effect I had hoped for when I had chosen them initially - people were caught up with some details of the problems that I felt detracted from what I could learn about set-oriented thinking. Therefore, I chose not to pursue those two problems in the second stage of interviews, and instead focused on the five problems discussed above.

Second, in the Stage 2 interviews I was open to recruiting more advanced students. While I was recruiting for Stage 1, I was somewhat careful not to simply find the top counting students, who might just easily and effortlessly solve the problems. In my analysis of Stage 1, though, I found that the problems were challenging for the students. While this difficulty was not overly problematic (their interviews still yielded rich data), I realized that it could be useful if some more advanced students ended up in my study. Further, given the fact that students did not overwhelmingly draw upon set-oriented thinking in Stage 1, I decided to recruit some students with more mathematical experience, particularly more experience with set theory. Therefore, as I recruited for Stage 2, and I had a number of senior level mathematics majors, and I ended up with a handful of students working on master's in mathematics, and even $\mathrm{PhD}$ students in mathematics and mathematics education. I was curious whether by interviewing such students, they might offer further insight into set-oriented thinking related to counting ${ }^{16}$.

\footnotetext{
${ }^{16}$ As it turns out, this change in recruitment from Stage 1 to Stage 2 was not very significant. I ended up with slightly higher level students (in particular I recruited a number of graduate students), but this did not seem to have a very big effect. The students with the most mathematical experience were not necessarily
} 
Third, as a result of my Stage 1 analysis, I prompted students more in Stage 2. In Stage 1 I wanted to see what students did on their own, and so even as we discussed their work and I gave them alternative solutions, I tried to refrain from directing them toward a particular course of action. Because I had developed some testable hypotheses in Stage 1, though, I decided to ask probing questions and even directly prompt students to redirect them toward a particular activity or strategy. I still had them work on their own and initially try the problem autonomously, but when we revisited the problem, I let myself ask questions that I thought could elicit set-oriented thinking. Even more, in Stage 2 I was not so much interested in whether or not students would use set-oriented thinking; rather, I wanted to get more information about how they might utilize such thinking. I asked such questions in a number of situations. First, if students had not already done so, at times I asked them to articulate particular elements they were trying to count. I did this because I saw the value that this conferred for people in Stage 1, and I suspected that identifying what they were trying to count could cause them to draw upon set-oriented thinking. I also believed that such interactions would increase students' dialog about sets of outcomes, and this would allow me greater insight into set-oriented thinking. And, related to this, I also occasionally used language that encouraged the coordination of sets and processes. For example, after I had given students ample time to work, if they felt were stuck, I might have asked a student to "identify a particular element that was 
counted more than once by this expression." This prompt most commonly arose when a student was evaluating two seemingly-similar processes, and I used it to test the conjecture that students could draw upon a particular outcome in order to explain why a given expression was incorrect. Again, I wanted to learn more about ways in which students utilized set-oriented thinking, and I was curious about the extent to which identifying such a problematic outcome could help students. Also, after students had done work on their own, I occasionally asked students to draw diagrams in Stage 2. I did this because diagrams often arose in Stage 1, and sometimes in powerful ways, and also because having students draw diagrams allowed for more time in which they could explain their thinking and reasoning. I felt that such activity could help me gain richer data as a researcher, and also could shed light on students' set-oriented thinking, especially in cases in which the diagram was meant to represent some aspect of the set of outcomes. I typically waited until students had done work on their own, and then I asked if there was a diagram or picture they could draw. In my prompting, I was sometimes specific (such as asking for a Venn diagram), but other times I simply asked students if there was any picture or diagram they were using.

These were some of the kinds of prompts came up in Stage 2 as a result of the analysis I had done on the Stage 1 data. On the whole, these prompts were designed not to disrupt the students' work, and I tried to prompt only after students had worked and had been free to try a variety of techniques. These prompts served to provide me as a researcher with opportunities to observe students' language and activity surrounding setoriented thinking. This concludes my discussion of my analysis of the Stage 1 data and 
the ways in which that prepared me for Stage 2. I now discuss the specifics of Stage 2. I will go on to discuss the overall analysis that followed.

\subsection{3 - Data collection and analysis of Stage 2}

In Stage 2, I interviewed 14 students one time each, and as mentioned above these interviews had been streamlined to accommodate both students' work on five problems and their evaluation of alternative answers to some subset of those problems. The Stage 2 interviews ranged from 60 to 90 minutes in length. I chose not to write content logs for Stage 2, but instead I immediately began to transcribe the interviews. This decision was made primarily because I did not have to plan for a second interview with the same student, and I was thus not in a hurry to analyze the data to prepare for another interview. I was very satisfied with the Excel format of the transcripts that I had developed during Stage 1 analysis, so after the Stage 2 interviews were transcribed I created Excel workbooks for each of the Stage 2 students, and before long I had the data from all 14 Stage 2 students (along with the 8 from Stage 1) organized in spreadsheets.

When I had the Stage 2 data available to work with, I initially went through what I might describe as a hybrid of open and axial coding. It was similar to open coding in the sense that I did go through the transcripts, examining what was happening in the text. I did not, however, carefully examine the transcripts line by line to the extent that I had in Stage 1. I was no longer looking to code particular utterances as being set-oriented or process-oriented. I focused instead on sentences and paragraphs that the students said, and on even longer exchanges between the students and me. As I mentioned above I was 
in the process of shifting toward emphasizing set-oriented thinking, so I highlighted instances that I took as examples of a student using set-oriented thinking. So while I did not code each utterance, I did go through and carefully read the transcripts and in some cases re-watched the video, paying particular attention to episodes that involved setoriented thinking. In some ways this coding process felt as though I was, on a broader level, organizing some of the phenomena I had found in my Stage 1 analysis, and this suggested elements of axial coding.

At this point I was still developing and articulating a working definition of setoriented thinking, but the identification of what I interpreted to be examples of setoriented thinking was helping me to refine the definition. Also, I suspected that the coordination of sets and processes might have been an important feature of students' setoriented thinking. As I went through the transcripts and identified particularly noteworthy situations, I familiarized myself with the data and tried to highlight times in which I observed what I interpreted as students coordinating sets and processes. At this time I was working with the entire body of data (including both Stage 1 and Stage 2), and I began to develop effective ways of analyzing both rounds of data together, which I discuss in the following section.

\subsection{4 - Overall analysis the entire body of data}

I now give details of how I went about coding the entire corpus of data, which included both Stage 1 and Stage 2 data. In particular, I outline the analysis that directly affected the development of the results and conclusions of my study, and particularly the 
analysis that supported the writing of both of the Results chapter and the Conclusions chapter. I first list the 4 steps in my analysis to give the reader a sense of how this section is organized, and I proceed to elaborate those steps in the pages to follow.

- Step 1: Identifying episodes in the data that suggested relevant themes related to setoriented thinking.

- Step 2: Using emerging themes from Step 1 to select episodes for the Results chapter, and using careful analysis of such episodes to construct the narrative in the Results chapter.

- Step 3: Using the themes in the Results chapter to develop a Coding Scheme to validate and contextualize the results.

- Step 4: Drawing upon the results from Steps 2 and 3 in order to draw conclusions.

As I will show in subsequent sections (as I discuss data analysis and present findings from my study), there were a number observable aspects of students' work that I took as evidence that a student was considering a set of outcomes. While some of this discussion will make more sense after subsequent sections arise (once sets of outcomes are more clearly articulated, for example), I present a general discussion here in the context of my data analysis. As mentioned in Section 3.5.2.2 above, sometimes students used settheoretic language in their counting, and such language often indicated to me that students drew upon set-oriented thinking. More often than not, however, students did not use such set-theoretic language, and I was left examining their language and activity to determine when they considered sets of outcomes. As I interviewed students and began to 
analyze data, I found that there were some common synonyms for some of the settheoretic terms described in Section 3.5.2.2. For example, instead of "elements" students might use "possibilities" or "options," and instead of "disjoint" a student might say that there is "no overlap between groups." Also, sometimes strategies, actions or gestures acted as indicators of students' considerations of set-oriented thinking. Instead of saying "complement" a student might have used a total-minus-bad strategy (discussed in more detail in Section ??), or instead of saying "partition" a student may have broken the set of outcomes into distinct cases. And, too, rather than explicitly talking about the importance of an element of the set of outcomes, a student may have simply provided a useful example of such an outcome. Thus, there were a variety of words and actions that I took as evidence of students drawing upon sets of outcomes. In subsequent sections when I talk about specific ways in which students used set-oriented thinking, I will more clearly elaborate what I took as evidence of such thinking.

\subsubsection{1 - Step 1: Identifying episodes in the data that suggested relevant themes related} to set-oriented thinking. As Auerbach and Silverstein (2003) propose, the methodological lens of grounded theory allows for the development of themes and ideas from the raw data. In this step of my analysis, I was identifying bits of relevant text and was drawing from them repeated ideas which ultimately lead to themes, to use Auerbach and Silverstein's language. Table 4 below reflects Auerbach and Silverstein's (p. 43) phases for developing theory from raw text. In this Step 1 of my analysis I was engaging in phases 2 and 3 in the table below - selecting relevant text and making note of repeated ideas. 


\section{MAKING THE TEXT MANAGEABLE}

1. Explicitly state your research concerns and theoretical framework.

2. Select the relevant text for further analysis. Do this by reading through your raw text with Step 1 in mind, and highlighting relevant text.

HEARING WHAT WAS SAID

3. Record repeating ideas by grouping together related passages of relevant text.

4. Organize themes by grouping repeating ideas into coherent categories.

\section{DEVELOPING THEORY}

5. Develop theoretical constructs by grouping themes into more abstract concepts consistent with your theoretical framework.

6. Create a theoretical narrative by retelling the participant's story in terms of the theoretical constructs.

\section{Table 4}

Specifically, this involved going through the transcripts and flagging episodes that I felt highlighted instances of set-oriented thinking. Guided by my initial passes through the data, and also the coding from Stage 1, I went through the transcripts, re-watching the video at times, and flagged episodes that exemplified students' uses of set-oriented thinking. As mentioned in the previous section, as I went through the transcripts I was not coding every single statement or line, but rather I highlighted those exchanges that had to do with set-oriented thinking. This process involved elements both of open and axial coding, but because I was making connections between concepts that I had already partially developed (ways in which students used of set-oriented thinking), I would characterize this activity as axial coding.

As a set of criteria for deciding which episodes to choose, I made the following considerations. First, I looked for those episodes that particularly had to do with my existing notions of ways in which students might utilize set-oriented thinking. This included any of the kinds of set-oriented thinking I had started to see in my analysis of 
Stage 1, including students' uses of case breakdowns, students' uses of a total-minus-bad strategy, and students' appeal to a particular element for the sake of determining whether an overcount had occurred. If students' discussions or activities involved any of these particular aspects, I would analyze the situation carefully to see whether or not a student was actually attending to the set of outcomes in some way. That is, there were times in which a student might have mentioned a case breakdown which might not have been clearly related to the set of outcomes; such an episode would not meet the criteria. While I initially looked for language and activity that suggested activity related to the hypotheses from the analysis of Stage 1, I also examined the episodes closely to determine whether I thought set-oriented thinking was occurring. I wanted to flag only those instances in which students' clearly (though it could have been implicitly) attended to the set of outcomes. Second, I also looked for instances in which a student was coordinating sets and processes. By this I mean that I paid attention to language or activity that suggested that a student related a particular counting process and a particular outcome. This could have been in through attributing a particular outcome to some counting process, or through indicating that a counting process had generated some particular outcome. Examples of such coordination are more fully described in Section 4.1, but this was an activity that suggested to me set-oriented thinking. The criteria for selection of an episode in Step 1 certainly had to do with students' language (as mentioned in Sections 3.5.2.2 and 3.5.4 above), but it could have been the case that 
students' activity or gesture indicated either one of the observed ways in which students' used set-oriented thinking or the coordination of sets and processes ${ }^{17}$.

As an example of this step of analysis, in Figure 5 I present part of the spreadsheet I had made for Makaena's work on the Test Questions problem. Coloring a part of the transcript purple meant that I flagged the episode as being significant, and that I specifically took it to be related to a coordination of sets and processes; the green indicated a place in which I had prompted in the original interview. In this instance, in addition to flagging the episode, I put notes down about what I felt was happening; I will explain my rationale below. To put the episode in context, Makaena was trying to determine why an incorrect expression, $\left(\begin{array}{l}5 \\ 2\end{array}\right) \cdot\left(\begin{array}{l}8 \\ 3\end{array}\right)$ was wrong.

\footnotetext{
${ }^{17}$ I will note, too, that the interviews had been designed to put students in situations in which they might have drawn upon set-oriented thinking. In particular, I had designed the study to put students in situations in which they were evaluating alternative expressions and were comparing seemingly-similar processes, and I suspected that students might use set-oriented thinking in such situations by appealing to a particular outcome that was overcounted. While this did not affect my criteria for selecting episodes, and while I also looked carefully at other parts of students' activity for instances of set-oriented thinking, I suspected relevant episodes might have arisen as students revisited problems and evaluated alternative expressions.
} 


\begin{tabular}{|c|c|c|c|c|}
\hline 59 & & M: & $\begin{array}{l}\text { So let's say they did. } 1,2,3,4,5 \text { and then they really did } 7,8,9,10 \text {, } \\
\text { okay. So they're choosing two of these, so let's say } 2 \text { and, number } 2 \\
\text { and number } 4 \text {. Okay. Then they're picking } 3 \text { from the rest. Well, see } \\
\text { this, yeah this doesn't, uh, I, this could equally have been } 6 \text { and } 7 \text {, and } \\
\text { then I could have chosen, uh, } 8 \text { and } 2 \text { and } 10 \text { or something, and that } \\
\text { wouldn't satisfy the requirements, so, I think that this } 8 \text { is the problem, } \\
\text { is because then, let's say you choose two of these, well you should still } \\
\text { be able to pick some of these questions. }\end{array}$ & $\begin{array}{l}\text { trying to } \\
\text { coordinate, but not } \\
\text { successfully }\end{array}$ \\
\hline 60 & & E: & This is good, I'm just letting you keep thinking about it. & \\
\hline 61 & & M: & Yeah. Hmm. & \\
\hline 62 & $1: 00: 40$ & E: & $\begin{array}{l}\text { Yeah it's like, can you come up with something that gets counted twice } \\
\text { by that one that doesn't get counted twice by yours? }\end{array}$ & big prompt! \\
\hline 63 & & M: & $\begin{array}{l}\text { Mmm. Okay well if we chose, let's say this chose } 2 \text { and } 4 \text {. And then I } \\
\text { pick } 3 \text { from the rest. These are gone. I pick } 3 \text { from the rest. Oh, well if } \\
\text { I pick } 1 \text { and } 3 \text { and } 5 \text {, okay, well let's say I pick } 1 \text { and } 3 \text {, and then } 2 \text { and } \\
4 \text { and } 5 \text {. Ah, I think that works. Um, so this is the same set of } \\
\text { questions as this, and they could have both been chosen using this } \\
\text { method, and they would have been counted as separate, uh, ways to } \\
\text { answer the, or to do the exam. }\end{array}$ & $\begin{array}{l}\text { there we go! The } \\
\text { prompt helped! }\end{array}$ \\
\hline 64 & & E: & Okay. & \\
\hline
\end{tabular}

Figure 5

I interpreted that initially (line 59) Makaena had coordinated sets and processes, because she did seem to attribute particular outcomes (sets of test questions) to the counting process. She did not, however, pick a problematic example (even more, she picked a set of questions that did not satisfy the constraint of the problem), and she was thus not able to use that coordination in order to justify why the incorrect answer was wrong; in my notes to myself I thus called her coordination 'unsuccessful. ${ }^{18}$, In line 63 , though, after I had prompted her, we see that she was able to identify a problematic outcome that was overcounted by her incorrect answer. Her language "so this is the same set of questions as

\footnotetext{
${ }^{18}$ This was a note to myself, and it is not entirely precise. Her coordination in line 59 was successful insofar as it was a coordination of sets and processes, but it did not ultimately help her in her work on the problem. I used the word "unsuccessful" to distinguish it from the coordination in line 63 which did allow her to successfully explain why the expression was incorrect.
} 
this, and they could have both been chosen using this method, and they would have been counted as separate...ways to...do the exam" indicated to me that she coordinated a counting process with an element of the set of outcomes, and this enabled her ultimately to see an error in the incorrect expression. Because this example indicated to me an instance of coordinating sets and processes, and this was an episode that I highlighted as I went through and flagged instances of set-oriented thinking.

What emerged from this Step 1 of my analysis, then, were a number of episodes that particularly highlighted instances of set-oriented thinking. These episodes were the product of the analysis I had done using a lens of grounded theory, and they provided repeating ideas from which I could eventually develop themes.

\subsubsection{2 - Step 2: Using emerging themes from Step 1 to select episodes for the Results} chapter, and using careful analysis of such episodes to construct the narrative in the Results chapter. After I had identified episodes that involved instances of set-oriented thinking, I prepared to select examples of students' work that would be presented in my Results chapter. In the Results chapter I primarily wanted to highlight set-oriented thinking, and I also wanted to give the reader a sense of how the interviews went and some of the different approaches that students took to the problems. To do this, I decided that I would organize the chapter by problem, giving details of three students' work on the problem and including other noteworthy episodes ${ }^{19}$. I thus needed to select which students I would discuss in detail for each particular problem. I knew that I wanted

\footnotetext{
${ }^{19}$ This is explained in greater detail in the Results chapter.
} 
compelling episodes that would highlight the ways in which set-oriented thinking arose, and I also wanted to give a sense of what occurred in the interviews. I chose episodes to go in the Results chapter based on how well I thought they would reflect instances of setoriented thinking. My goal in selecting episodes was not to get the most common kinds of activity with which students engaged, but rather to find those episodes that were most interesting and that were related to set-oriented thinking.

In order to do this, I again drew upon principles of grounded theory, particularly Auerbach and Silverstein's (2003) phase of developing themes from repeated ideas. Specifically, I first looked back through those flagged episodes from Step 1. Then, for each problem I made note of those episodes that exemplified the various ways of using set-oriented thinking, and I tried to choose a set of student episodes that would, as whole, give examples of the kinds of set-oriented thinking I had observed. As I began to narrow those episodes that I wanted to include in the Results chapter, I carefully examined each of them to explicate the role of set-oriented thinking in the students' combinatorial problem solving. Through this I started to get a better sense of what set-oriented thinking entailed. I also looked through students' work to create a set of examples that could highlight the various "best" instances of set-oriented thinking. The end product of this phase of analysis was those cases presented in the Results chapter. While Strauss and Corbin (1998) do not give precise language for this kind of activity, I would characterize it as being somewhere between axial and selective coding. By examining and selecting particularly enlightening instances of set-oriented thinking, I was organizing concepts into broader categories, which suggests axial coding. The organization that was being 
done, though, was on higher-level concepts than I had organized previously, and so this step felt closer to theory development than Step 1 had. Additionally, beyond organizing themes, there was a narrative element of this aspect of my analysis as well. During this time I was reconstructing the story of what happened as the students engaged with the problems in terms of my constructs related to set-oriented thinking.

As an example of this part of my analysis, I highlight Anderson's work on the Passwords problem; Anderson was one of the students I chose to discuss in detail on this problem. Below I will show how I flagged Anderson's work, but the excerpts should also serve to indicate that Anderson's work was representative of a couple of interesting episodes. He used the total-minus-bad strategy, and he also successfully drew upon a particular element of the set of outcomes. His work on this problem will be presented in much greater detail in the Results chapter, but this should give some sense of why his was an episode chosen to highlight as an important result.

First, in Figure 6 we see that I had flagged lines 114-116, and I felt that these provided an instance in which Anderson used set-oriented thinking in a total-minus-bad strategy. His language in these lines suggested that he counted the total and then subtracted off bad cases. 


\begin{tabular}{|c|c|c|c|}
\hline 112. & & $\begin{array}{l}\text { another, if I had one E in any location - so that's } 8 \text { times } 25^{\wedge} 7 \text {, and } \\
\text { then subtract the case where I have } 2 \mathrm{E}^{\prime} \text { 's in any location? Um, is this } \\
\text { right? Because I have...that would be (inaudible) possibilities for } 2 \text {. } \\
\text { Um, okay, let's see. What l'm trying to do right now is I'm trying to } \\
\text { work at the problem backwards. }\end{array}$ & \\
\hline 113 & & E: Okay. & \\
\hline 114. & & $\begin{array}{l}\text { A: Um. where I take the total number of possible 8-letter } \\
\text { combinations. }\end{array}$ & \\
\hline 115 & $00: 21: 47$ & E: Yeah. & \\
\hline 116 & & $\begin{array}{l}\text { A:And then I subtract the cases where the password doesn't meet our } \\
\text { criteria of having at least } 3 \text { E's. Um, so in terms of total passwords, } \\
\text { there's, uh, } 26^{\wedge} 8 \text { total combinations, 'cause there's } 26 \text { letters, and } \\
\text { there's } 8 \text {, uh, it's } 8 \text { characters, or } 8 \text { letters. And then the case where } \\
\text { one of them would be an E, or the case where none of them would be } \\
\text { an } E \text { is, um, } 25^{\wedge} 8 \text { characters because it's the same - it's any letters } \\
\text { for all } 8 \text {, and there's } 25 \text { letters total. Then the next case where there's } \\
\text { only one E, the E can only be in one of } 8 \text { locations, multiplied by uh, } \\
\text { the remaining } 7 \text { positions filled by any of the } 25 \text { letters, so that's } 25^{\wedge} 7 \text {, } \\
\text { subtracted by another - in the case where there's } 2 \text { E's, which for } \\
\text { some reason is not coming to mind, um, I don't know if I'm really } \\
\text { going to brute force this, I guess. Brute force is so inefficient. Um. } \\
\text { Well in the one case we have one, in the two case we have } 2 \text {, three } \\
\text { case we have } 3,4 \text { cases we have...Because in } 3 \text { cases we had } 3 \text {, but } \\
\text { in } 4 \text { cases we have } 9 \text { ? In } 5 \text { cases we would have another. } 4 \text {. What am } \\
\text { I missing here? I have - this is not coming to mind, um, }\end{array}$ & $\begin{array}{l}\text { uses total - bad as } \\
\text { an alternative way } \\
\text { to approach the } \\
\text { problem!! }\end{array}$ \\
\hline 117 & & E: Okay, that's okay. & \\
\hline 118 & & A: Yeah, I'm not, I quess my brain's not on today. & \\
\hline 119 & & $\begin{array}{l}\text { E: So, I have a quick - so I see, I see you're maybe considering that } \\
\text { opposite way of looking at it, that maybe that will give you some }\end{array}$ & \\
\hline
\end{tabular}

Figure 6

We also see that later in the transcript I again flagged Anderson's work as having involved set-oriented thinking (Figure 7) by shading relevant episodes. This time Anderson identified a particular password (the all E's password) that had been counted more than once by the incorrect expression. This excerpt is also particularly insightful because we see that it captures the fact that he could see how both expressions might make sense (line 188), but he was able to talk about how the incorrect expression produces the all E's password more than once. 


\begin{tabular}{|c|c|c|c|}
\hline 184 & & A: Okay. & \\
\hline 185 & & $\begin{array}{l}\text { E: And let's say, again, a high school student or something comes } \\
\text { and says, I can't tell which of these is right, }\end{array}$ & \\
\hline 186 & & A: Okay. & \\
\hline 187 & & $\begin{array}{l}\text { E: Can you talk through how you would - how you'd explain to them } \\
\text { which is right, and, }\end{array}$ & \\
\hline 188 & & A: Okay. Um. Well l'd say they both are very sound from a first view. & \\
\hline 189 & $00: 38: 53$ & E: Okav. & \\
\hline 190 & & $\begin{array}{l}\text { A: A first look. Uh, I mean they look, in your head they sound just } \\
\text { fine. So um, the problem, like I said, with this one with, um, } \\
\text { simplifying the problem here is that it simplifies it a little too much. } \\
\text { Um, if, because it says we can take - we can apply - we only need to } \\
\text { worry about } 3 \text { of the letters, those have to be E's, and the rest can be } \\
\text { anything we want, we can take the example where all of them are E's, } \\
\text { um, now because we treat } 5 \text { of those E's as just fluff, and it doesn't } \\
\text { really matter, then when we go back, and like sort of re-iterate the } \\
\text { problem, we can end up with the initial } 3 \text { E's that we had in different } \\
\text { locations, um, the ones that we have set. So say the first time we go } \\
\text { through the } 3 \text { E's are the first } 3 \text { letters of the password, second time } \\
\text { we go through the } 3 \text { E's are the last } 3 \text { letters of the password. Since } \\
\text { the other } 5 \text { letters can be any of the } 26 \text { letters, let's say we make } \\
\text { them all E's again. Well the password is exactly the same in both } \\
\text { cases, but this solution would count them twice. Um, because it } \\
\text { doesn't really care about the } 5 \text { non-E's. or } 5 \text { not set E's. }\end{array}$ & BINGO!!! \\
\hline \multirow[t]{2}{*}{191} & & $\mathrm{E}: \mathrm{Mm}-\mathrm{hmm}$. & \\
\hline & & $\begin{array}{l}\text { A: Um, the first problem wants to say, let's try not to over-lap } \\
\text { ourselves here, and split them into distinct cases. So let's say we } \\
\text { have exactly } 3 \text { E's. How many ways can we arrange } 3 \text { different E's in, } \\
\text { you know, an 8-letter password. For the remaining } 5 \text {, we'll just, you } \\
\text { know, they can be any combination of letters, it really doesn't matter, } \\
\text { as long as they're not E's, because then we start crossing into other } \\
\text { cases, uh, so the next case would be, let's have exactly } 4 \text { E's, how } \\
\text { many ways could we arrange that, and then the remaining } 4 \text { letters }\end{array}$ & \\
\hline \multicolumn{2}{|c|}{$14+1$} & asswords Cards Groups Students Even Numbers Test Questio & ons Apples\&C \\
\hline
\end{tabular}

Figure 7

Having chosen particular students' work on particular problems, I then began to write the Results chapter. Doing so provided another aspect of analysis in itself. In the Results chapter, I elaborated more than just the particular instances of set-oriented thinking (I also included other kinds of insights or approaches that students took), but I attempted to emphasize students' uses of set-oriented thinking as much as possible. Specifically, I described the students' work on the problem, and I included those excerpts that I thought 
fleshed out the feel of the interviews. As I constructed my narrative of the Results chapter, themes began to emerge, and these are outlined in Table 5 in the following section. While I had certainly been attuned to these ideas through my analysis thus far (some of these were included as hypotheses from Stage 1), the writing of the Results helped my thinking about these ideas to be more concrete.

\subsubsection{3 - Step 3: Using the themes in the Results chapter to develop a Coding Scheme}

to validate and contextualize the results. There was another important aspect of my analysis that came out of the writing of the Results chapter and the development of themes, which I will describe now. In addition to flagging episodes that reflected ways in which students used set-oriented thinking, my analysis of the data involved another analytical method. Even though I was doing a qualitative study and was using grounded theory to develop themes and hypothesis, I wanted some coding scheme that could help to ensure that my findings were not overly dependent on my own interpretation of the data. Additionally, I thought that coding the data as a whole and getting a sense of the numerical tallies for certain occurrences of phenomena would help me to determine and describe the typicality of the particular episodes I had chosen to include in the Results chapter. I thus developed a Coding Scheme that I hoped would both validate my thinking about the students' mathematics and that could serve to contextualize the episodes I had chosen to include as results.

3.5.4.3.1 - Identifying themes to include in the Coding Scheme. The Coding Scheme is organized according to particular documents that contained a series of yes/no questions that a coder could answer, which I refer to as "schedules." I provide examples of such a 
schedule in Figure 8 below to give the reader a context for the following discussion. Figure 8 shows Schedule A, which is a schedule related to students' uses of case breakdowns.

\begin{tabular}{|c|c|c|c|c|c|c|}
\hline \multicolumn{7}{|c|}{ Schedule A-Case Breakdown } \\
\hline \multirow[t]{4}{*}{ Check } & \multirow{2}{*}{\multicolumn{3}{|c|}{$\begin{array}{l}\text { Is this case breakdown in the context of a } \\
\text { smaller case? }\end{array}$}} & Lines & U/P & Comments \\
\hline & & & & & & \\
\hline & \multicolumn{3}{|c|}{$\begin{array}{l}\text { Is the smaller case is omorphic to the } \\
\text { original problem? }\end{array}$} & & & \\
\hline & \multicolumn{3}{|c|}{$\begin{array}{l}\text { Does the student relate the work in the } \\
\text { smaller case back to the original problem? }\end{array}$} & & & \\
\hline \multicolumn{7}{|c|}{ Does the student: } \\
\hline \multirow[t]{3}{*}{ Check } & & & & Lines & $\mathrm{U} / \mathrm{P}$ & Comments \\
\hline & \multicolumn{3}{|c|}{$\begin{array}{l}\text { Refer to (but not implement) the case } \\
\text { breakdown? If so, go to } \mathrm{Al} \text {. }\end{array}$} & & & \\
\hline & \multicolumn{3}{|c|}{$\begin{array}{l}\text { Implement the case breakdown? } \\
\text { If so, go to } \mathrm{A} 2 \text {. }\end{array}$} & & & \\
\hline \multicolumn{7}{|c|}{$\frac{\mathrm{Al}}{\text { In their reference to the cas e breakdown, is there evidence that }}$} \\
\hline \multirow[t]{4}{*}{ Check } & \multirow{3}{*}{\multicolumn{3}{|c|}{\begin{tabular}{|l|} 
The case breakdown is correct? \\
(just the cases, not necessarily the counts) \\
The case breakdown is incorrect?
\end{tabular}}} & Lines & $\mathrm{U} / \mathrm{P}$ & \\
\hline & & & & & & \\
\hline & \multicolumn{2}{|c|}{$\begin{array}{l}\text { The cas e breakdown is incorrect? } \\
\text { (just the cases, not neces sarily the counts) }\end{array}$} & & & & \\
\hline & \multicolumn{3}{|c|}{ No basis for judgment. } & & & \\
\hline \multicolumn{7}{|c|}{$\frac{\mathrm{A} 2}{\text { In their implementation of the case brealdown }}$} \\
\hline \multirow{4}{*}{\multicolumn{2}{|c|}{\begin{tabular}{|l|l|} 
Yes & No \\
\end{tabular}}} & & & Lines & $\mathrm{U} / \mathrm{P}$ & Comments \\
\hline & & \multicolumn{2}{|c|}{ Is the case breakdown correct? } & & & \\
\hline & & & Are the cases disigint? & & & \\
\hline & & & $\begin{array}{l}\text { Is the breakdown } \\
\text { complete? }\end{array}$ & & & \\
\hline & & & ases counted correctly? & & & \\
\hline
\end{tabular}

Figure 8

I will elaborate the various schedules in detail in the following section, and the schedules themselves (as well as descriptions of and rationale behind the individual schedules) can be found in Appendix B. Additionally, I give more detail on the development of the Coding Schemes and their role in validity and reliability in Sections 
3.5.4 and 3.5.5. Also, I decided to make two separate Coding Schemes, one for the students' original work on the problem, and one for when they revisited the problem and evaluated alternative answers. I did this because as I developed the Coding Scheme, it became clear that there were different kinds of information I wanted in each type of situation; I felt that it would have been inefficient had I tried to incorporate questions for both kinds of information into one Coding Scheme. I will refer to the coding scheme that was used for students' initial attempts at the problems as Coding Scheme 1, while the coding scheme used to describe the times when students revisited a problem is called Coding Scheme 2.

The development of the specifics of the Coding Scheme was rooted in my analysis to that point. In particular, the themes in Table 5 had already emerged as prominent ways in which students used set-oriented thinking, and these were thus the ideas that I wanted to code for as I developed the Coding Scheme. Table 5 reflects the ways in which the themes showed up in the schedules of the Coding Scheme, and I explain the rationale for each element of the coding scheme in the following section.

\begin{tabular}{|l|l|}
\hline $\begin{array}{l}\text { Themes that may suggest set-oriented } \\
\text { thinking }\end{array}$ & Schedules that got at that theme \\
\hline Case Breakdowns & $\begin{array}{l}\text { Coding Scheme } 1-\text { Schedule A } \\
\text { Coding Scheme } 2-\text { Schedule A }\end{array}$ \\
\hline Total-Minus-Bad Strategy & $\begin{array}{l}\text { Coding Scheme } 1-\text { Schedule B } \\
\text { Coding Scheme } 2-\text { Schedule A }\end{array}$ \\
\hline Appealing to Particular Outcomes & $\begin{array}{l}\text { Coding Scheme } 1-\text { Schedule D } \\
\text { Coding Scheme } 2-\text { Schedule C }\end{array}$ \\
\hline Coordinating Sets and Processes & Coding Scheme 2 - Schedule A, B, C \\
\hline
\end{tabular}




\begin{tabular}{|l|l|}
\hline Diagrams $^{* 20}$ & $\begin{array}{l}\text { Coding Scheme 1 - Schedule F } \\
\text { Coding Scheme 2 }- \text { Schedule D }\end{array}$ \\
\hline Multiplication Principle* & Coding Scheme 1-Schedule C \\
\hline
\end{tabular}

Table 5

I elaborate these phenomena in greater detail in the Conclusions chapter, and in fact some of my main conclusions involve fleshing out the ways in which students utilized set-oriented thinking. I discuss them here briefly as a way to describe how the development of the Coding Schemes was based on emerging themes related to students' set-oriented thinking.

3.5.4.3.1.1 - Case breakdowns. In my analysis of the data to that point, I had observed students breaking up their set of outcomes in some way. Sometimes they used settheoretic language (such as "partitions" or "outcomes") in order to describe their work, but more often they referred to this activity as involving "cases." I thus suspected that students' language and activity surrounding cases could be tied to their understanding of the set of outcomes, and I thus made schedules in both Coding schemes that addressed cases. There are two aspects of cases I tried to capture in these schedules. First, I wanted to allow for a distinction between when students implemented case breakdowns, or when they mentioned but did not execute a case breakdown (I felt that even a mention of a case breakdown could signify some set-oriented thinking, and I did not want to focus only on

\footnotetext{
${ }^{20}$ Diagrams and Multiplication Principle were two themes that I had not yet seen as being as explicitly tied to set-oriented thinking as the others were. Nonetheless they represented common phenomena that had arisen in my initial analysis, and I included them in the schedules both because of their potential relationships to set-oriented thinking and because they arose in the data quite frequently.
} 
the implementation of a case breakdown). Second, the two most important mathematical aspects of a case breakdown are that the cases should partition the set of outcomes into disjoint subsets whose union is the entire set of outcomes. I wanted to consider these aspects separately from how the students then actually counted the cases. I thus designed the schedule to allow coders to identify situations in which a student got a problem wrong, but in which they had still correctly determined an appropriate way to break the problem into cases.

3.5.4.3.1.2 - Total-minus-bad strategy. Another phenomenon that suggested students used set-oriented thinking was the "total-minus-bad" strategy, in which students counted the total number of outcomes and subtracted off those outcomes that did not satisfy a particular constraint. I interpreted such activity as reflecting set-oriented thinking, and I thus made a schedule for the total-minus-bad strategy. Specifically, in the schedule I made a distinction between students' reference to and implementation of the total-minusbad strategy. I also made a distinction between how students articulated the total-minusbad relationship and how they actually counted the "total" and the "bad." If a student was going to get a problem wrong when they used a total-minus-bad strategy, I wanted to know if it was because they incorrectly considered total-minus-bad (which could say something about their set-oriented thinking, perhaps) or if they correctly implemented total-minus-bad but incorrectly counted the parts. I also included a question about the principle of inclusion/exclusion because it is a more explicitly set-theoretic version of total-minus-bad, and I was curious how and whether the principle of inclusion/exclusion would arise for students. 
3.5.4.3.1.3 - Appealing to particular outcomes. Another theme seemed to be that students would, on occasion, appeal to particular elements of the set of outcomes. This arose in various contexts; sometimes students did this to determine whether or not order mattered in a problem, and other times they did so to identify that a particular counting process had overcounted. I took instances in which students referred to or used particular elements of the set of outcomes as an instance of set-oriented thinking, and this was a feature that I wanted to include in my Coding Schemes. I made a schedule for Coding Scheme 1 that essentially asked questions about the ways in which students appealed to a particular outcome. Students occasionally appealed to particular outcomes as they revisited problems and compared two seemingly-identical solutions, and thus I made a particular schedule in Coding Scheme 2 that asked specific questions about students' activity related to identifying particular elements that had been overcounted.

3.5.4.3.1.4 - Coordinating sets and processes. Another emerging theme was that the coordination of sets and processes was an important aspect of students' set-oriented thinking. While I was still articulating what that entailed, at that time I was interested in instances in which students seemed to be attributing some outcome (or set of outcomes) to a counting process they were implementing. Given its apparent connection to setoriented thinking, I asked questions about the coordination of sets and processes in one of the schedules in Coding Scheme 1, and I made a schedule completely devoted to it in Coding Scheme 2.

3.5.4.3.1.5 - Drawing a diagram. I also made a schedule to get at students' uses of diagrams. As I was writing the schedules, there seemed to be the potential for diagrams to 
have served an important role in students' set-oriented thinking, and I thus wanted to provide an avenue to record the diagrams that students had used in their counting activity.

3.5.4.3.1.6 - Multiplication principle. There was another phenomenon that was extremely common among students, and that was to use language such as "slots" or "spots" and to refer to the number of choices they had for each slot. After my initial analysis I was not convinced that this was clearly related to set-oriented thinking, but because it arose so frequently, and because there could have potentially been a connection, I included a schedule for it in Coding Scheme 1. Additionally, because the slots language represents a way of considering the multiplication principle, in this schedule I also asked questions about students' other uses of the multiplication principle that I thought might have been related to set-oriented thinking, namely tree diagrams and Cartesian products.

This concludes the section on the schedules of Coding Schemes. In order to give the reader an example both of the Coding Schemes and of what analysis looked like using the instruments, I now go through two examples of students' work. First, I will describe Makaena's work on the Groups of Students problem, and I will show how her work was coded according to Coding Scheme 1. Where appropriate, I discuss excerpts that were included as evidence of a particular phenomenon. I have scanned in the schedules I coded, and I discuss each schedule below ${ }^{21}$. Also, as a note to the reader, in the schedules

\footnotetext{
${ }^{21}$ I chose to describe this problem because it was one that was double-coded by both another coder and me, and we had $100 \%$ agreement in our coding on this particular student and problem. Further description of the double coding is given in Section 3.5.5 on validity and reliability.
} 
there are places in which the coder can write in "line" numbers. These were intended so that if the coder answered yes to a given question about a given phenomenon, he or she should include the line numbers from the transcript as evidence of their decision. Each of the coders worked from the same transcripts in the same spreadsheets, and the "lines" refer to specific rows in the Excel spreadsheet in which the transcripts were organized. As noted above, the transcripts were organized so that each row represented one person's turn of talking; a change in row indicated a change in the speaker. Thus, when I refer to lines below (and when lines were given in evidence in the schedules) it could be the case that a single line signifies several sentences and ideas given by a single person.

\subsubsection{2 - Example of Coding Scheme 1}

Makaena's Front Page: 


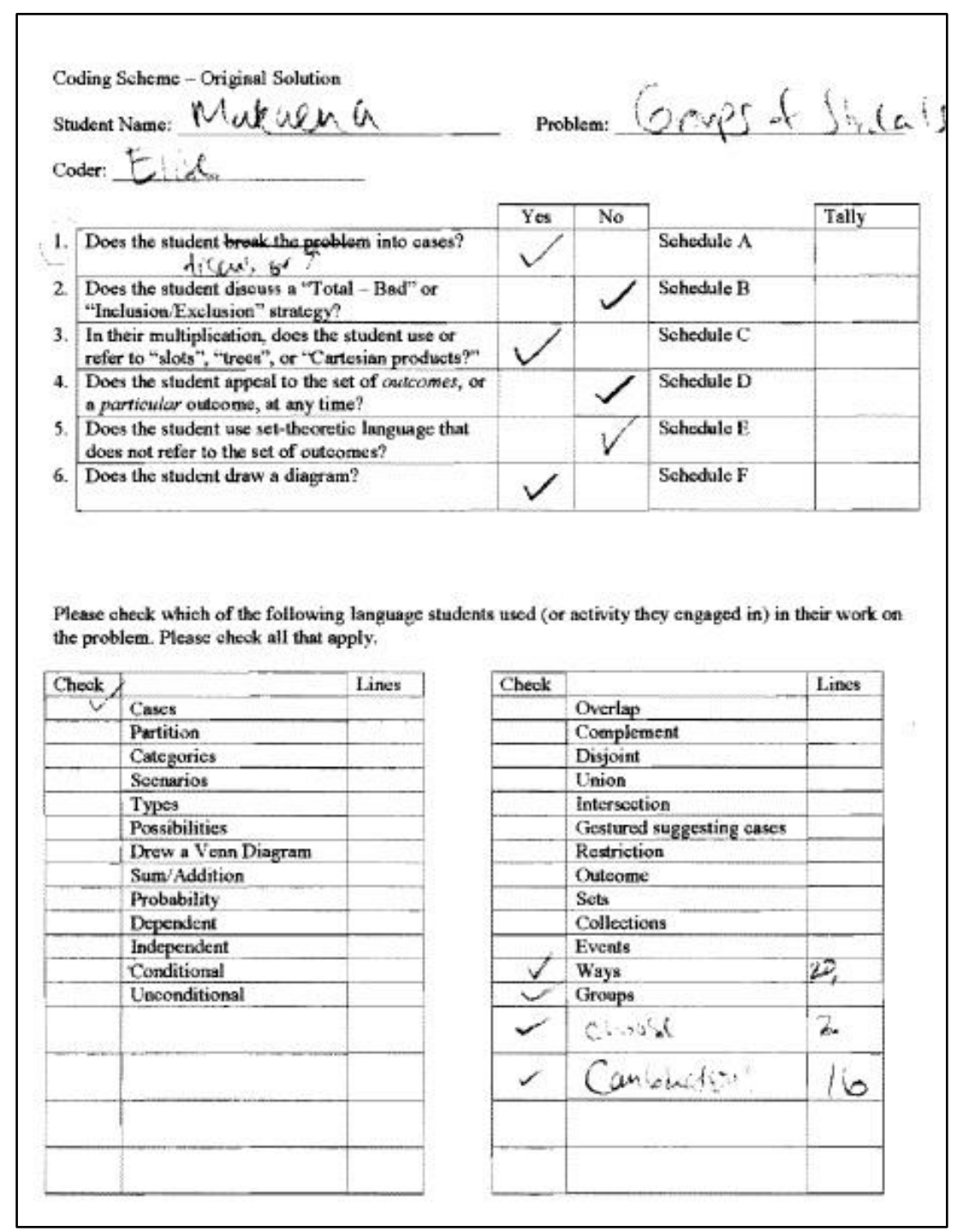

Figure 9

From the Front Page (Figure 9) we see that I filled out schedules A, C, and F (which represent case breakdowns, multiplication, and diagrams, respectively) for Makaena's work on this problem. Also, we see that she used the following language as some point in her work on the problem: cases, ways, groups, choose, and combinations. 
Below is my coding of Schedule A (Figure 10), which indicated that Makaena referred to or used a case breakdown in some way.

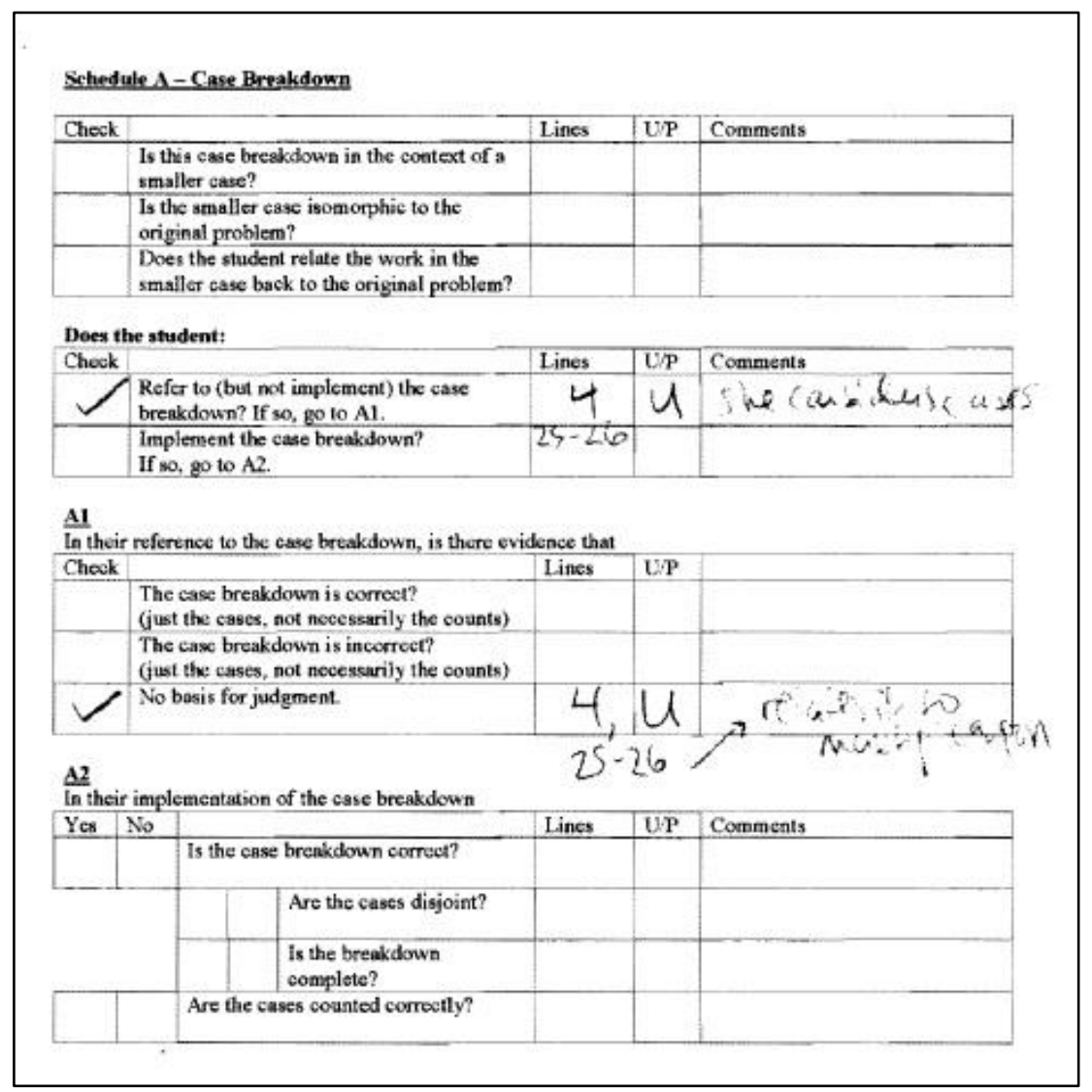

Figure 10

From Makaena's Schedule A on the Groups of Students problem, we see that I indicated that she referred to, but did not implement, a case breakdown. I cited line 4 to indicate this, and I noted that there was an additional mention of cases in lines 25-26 as well ${ }^{22}$. Line 4 reads:

${ }^{22}$ Lines 25-26 had included a brief mention of cases as a way of thinking about multiplication. I cited it in Schedule $\mathrm{A}$ as another instance in which she referred to a case breakdown but did not implement it. 
M: Well, I guess part of what I was thinking is that, there's, yeah I always want to break things down into kind of cases, and the cases are sort of a lot, and I had almost, I had almost wanted to try this with smaller groups,

Given her language above, I coded that while she certainly referred to (but did not implement) a case breakdown, there was no evidence in her language above for me to determine whether or not her case breakdown was correct. The $\mathrm{U}$ in the schedule means that her work in that line was unprompted; she brought up the case breakdown on her own without prompting from me.

Makaena's Schedule C was the next schedule on this problem.

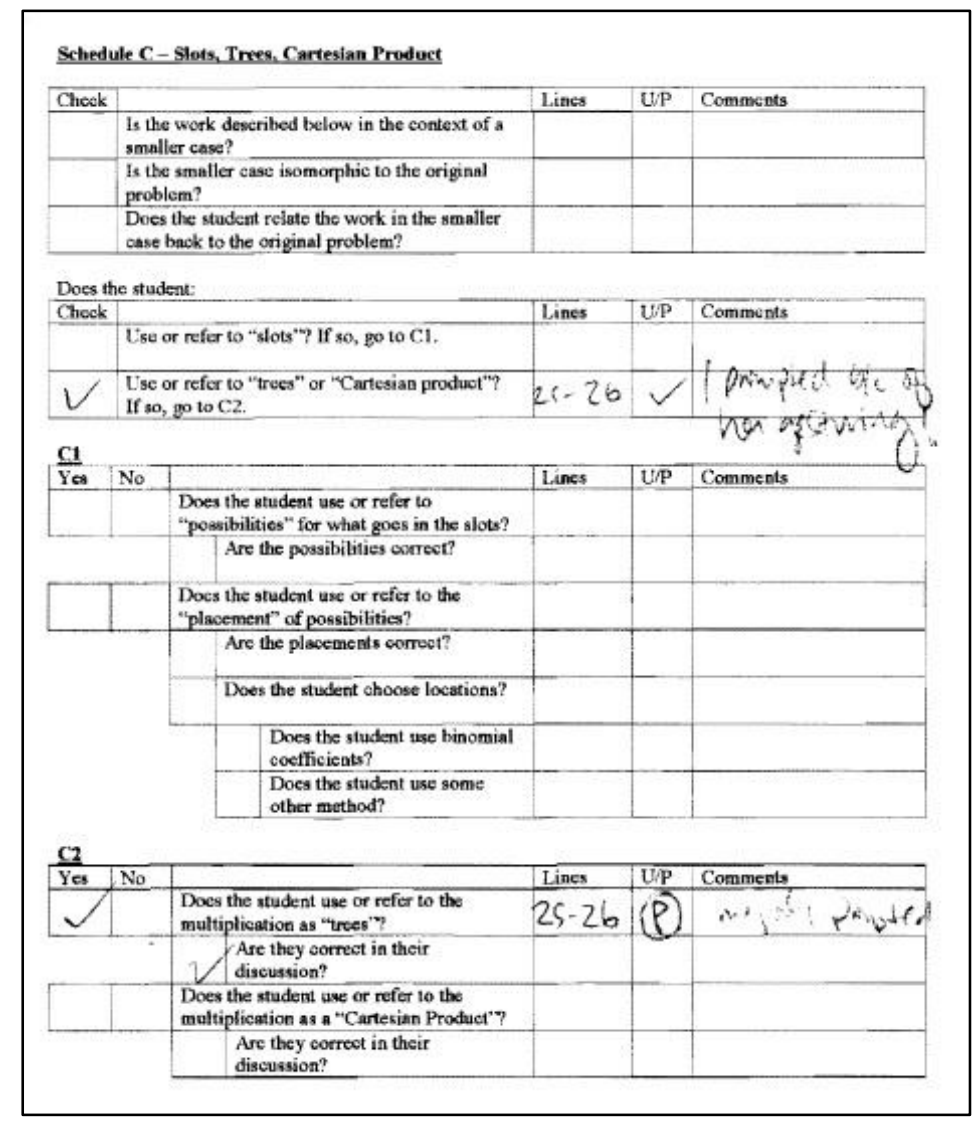

Figure 11 
In this schedule, while the other coder and I had the same boxes checked, I had cited lines 25-26 while he had cited line 30, so I include lines 25-30 below as evidence for this code. Both of us had indicated "Prompted."

E: Are you picturing like a tree kind of thing for that, or are you just matching things up, you had that (inaudible)

M: Yeah, I guess, the reason I multiply is that I'm thinking, okay, let's say I pick one of these ways... Well I've still got to do this. And then if I had, for each one of those, so each one of those ways, yeah, I guess it would be like a tree, but it's just sort of like, each one is its own little case.

My question about trees is the reason that I coded the episode as "Prompted." In response to my prompt she indicated that she considered trees, although she also said that she thought of cases as well. This mention of trees prompted the coding of Schedule $\mathrm{C}$ above.

Makaena had also drawn a diagram for the problem, and so I filled out a Schedule F (Figure 12). She had drawn during the excerpt in Line 2; to compare, I include a picture of her actual diagram as well (Figure 13).

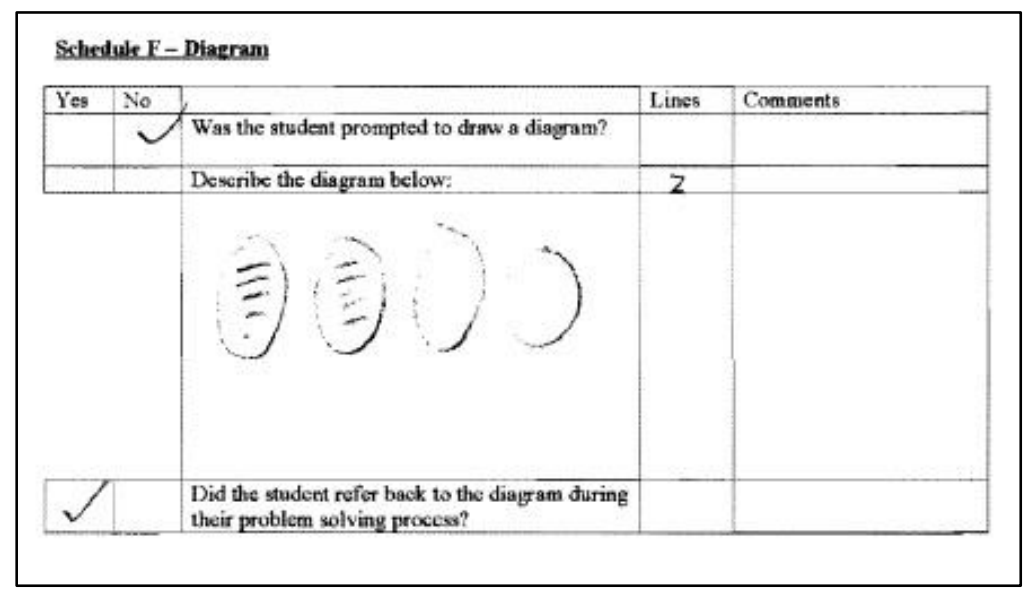

Figure 12 


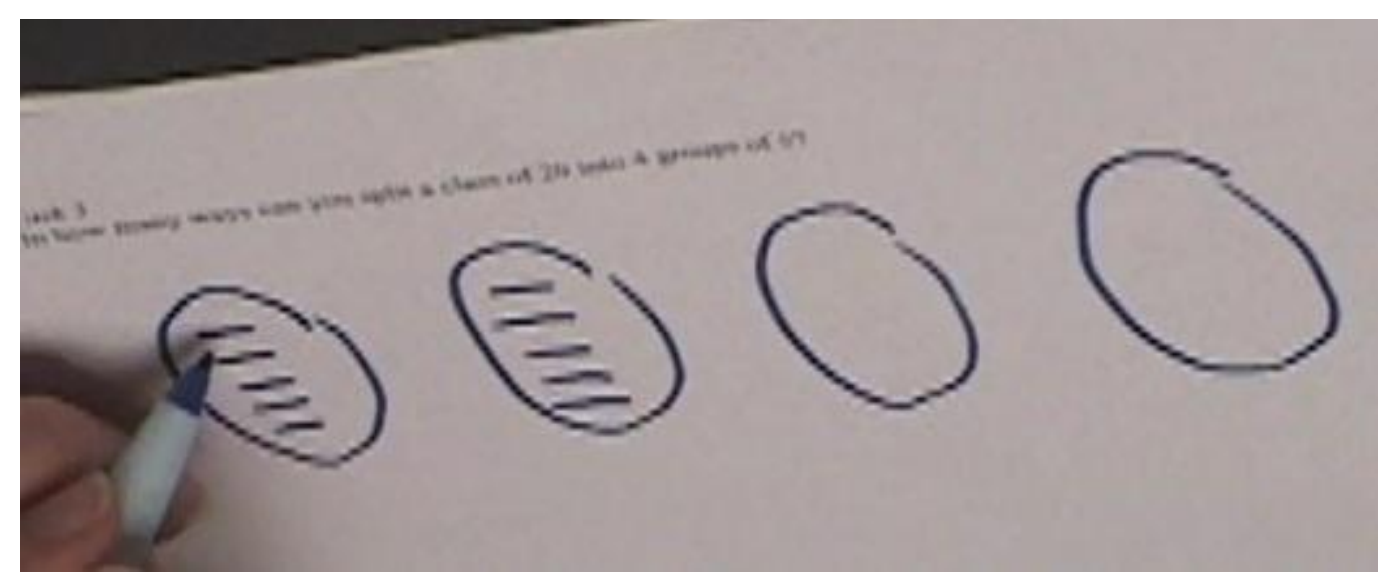

Figure 13

This discussion thus highlights how Makaena's work on the Groups of Students problem was coded. This above example was designed to get a sense of how the analysis occurred and the kind of evidence that was used in order to determine coding decisions. I will note that in Makaena's work on this problem, we do not see very much evidence of set-oriented thinking. She mentioned cases briefly, but she made no mention of sets of outcomes.

\subsubsection{3 - Example of Coding Scheme 2}

In order to give a further sense of the coding process, I provide an example of my codes for Brandon's work when he revisited the Groups of Students problem. This is from Coding Scheme 2. 


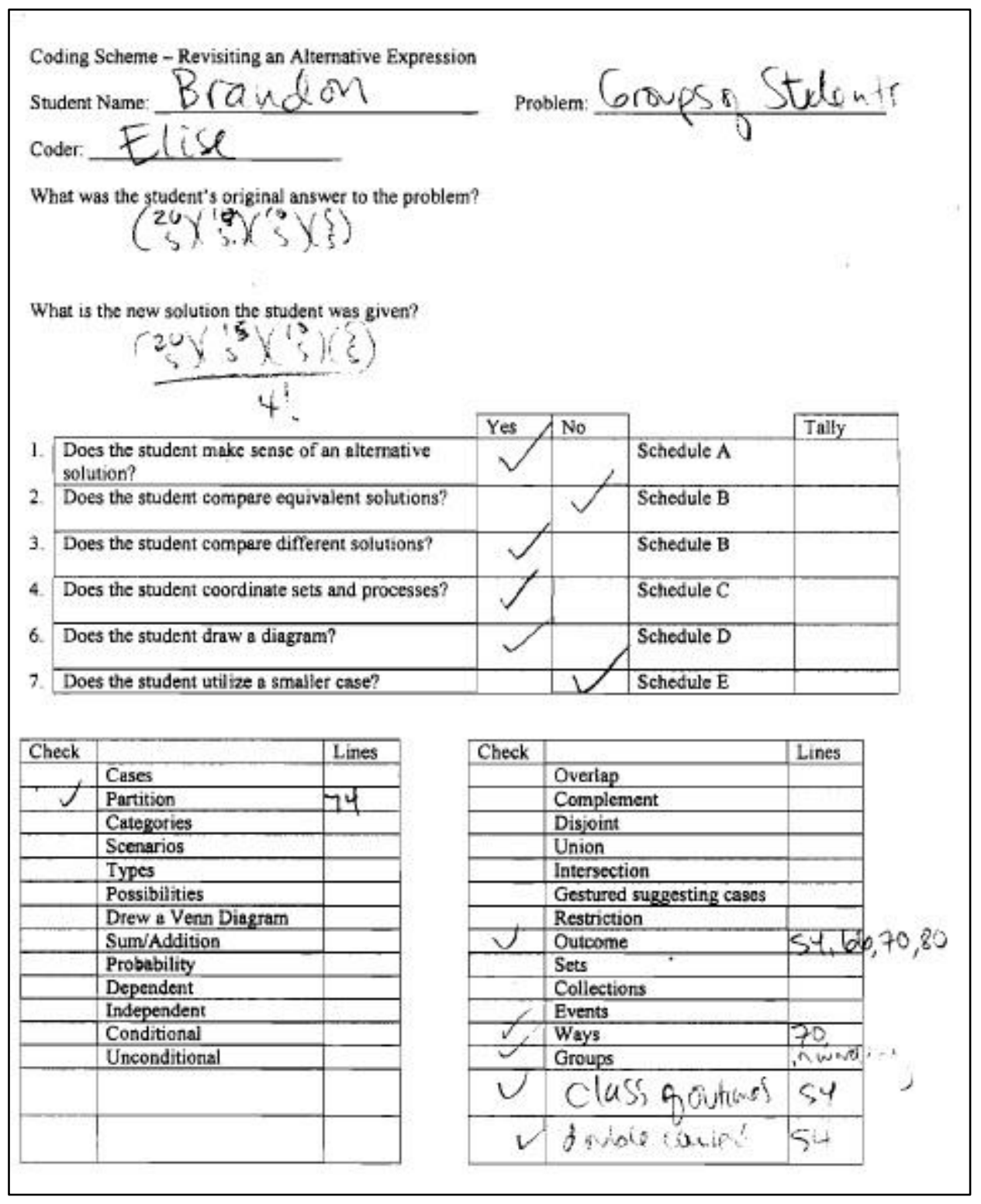

Figure 14

On Brandon's front page (Figure 14), Brandon's original solution to the problem had been $\left(\begin{array}{l}20 \\ 5\end{array}\right) \cdot\left(\begin{array}{l}15 \\ 5\end{array}\right) \cdot\left(\begin{array}{l}10 \\ 5\end{array}\right) \cdot\left(\begin{array}{l}5 \\ 5\end{array}\right)$, while the alternative answer he examined was 


$$
\frac{\left(\begin{array}{l}
2 \\
5
\end{array}\right) \cdot\left(\begin{array}{l}
15 \\
5
\end{array}\right) \cdot\left(\begin{array}{l}
10 \\
5
\end{array}\right) \cdot\left(\begin{array}{l}
5 \\
5
\end{array}\right)}{4 !} \text {. We also see that I filled out Schedules A, B, C, and D, and I had }
$$

noted that he used the words partition, outcome, ways, groups, class of outcomes, and double counted.

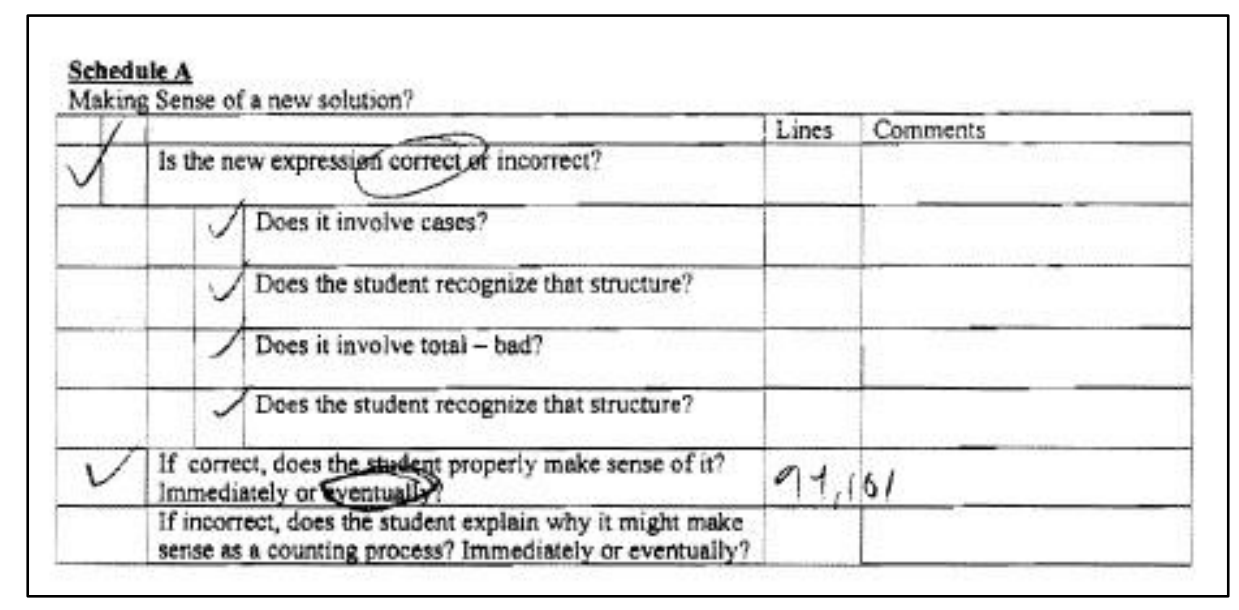

Figure 15

In Schedule A (Figure 15), I recorded that the new expression was correct and involved neither case breakdowns nor total-minus-bad. I gave Lines 94 and 101 as evidence that Brandon eventually made sense of the alternative answer, and I include Lines 94-101 below.

B: Yeah I think, I actually did it, like I was saying, I'd assign students to group 1, and then I think it naturally fell out, then I realized, oh, you know, if I do that in a different order, I say assign them to group 4 instead of group 1, I think what we're talking about earlier was where you asked me, um, are they doing something specific, like did you give them specific assignments, you go to recess, you go to lunch, something like that. Or are they just breaking up?

E: Mm-hmm.

B: Um, I think once I kind of remembered that, it kind of naturally fell out that, oh, it doesn't matter if I call them group 4 or group $1 \ldots$ They're doing the same 
thing, they're together, it's that these five students are grouped together, is the important thing, not what they're called, or what they're doing.

E: Okay, cool. Cool okay so you think that that answer gets at what the question is asking.

The above exchange suggests to me that Brandon was able to see why the division by 4 factorial was necessary in the problem. He realized that if he had the same group that was assigned in a different order, it would be the same group of people. He realized that only the make-up of the groups was important, and they did not need to be distinguished in any way.

Schedule B (Figure 16) has to do with what happened as Brandon compared alternative answers, and in this case he was comparing two different expressions, as described above. I have already included Lines 94-101, and I provide Lines 70-72 below, which I had used as evidence of Brandon appealing to a particular element. 


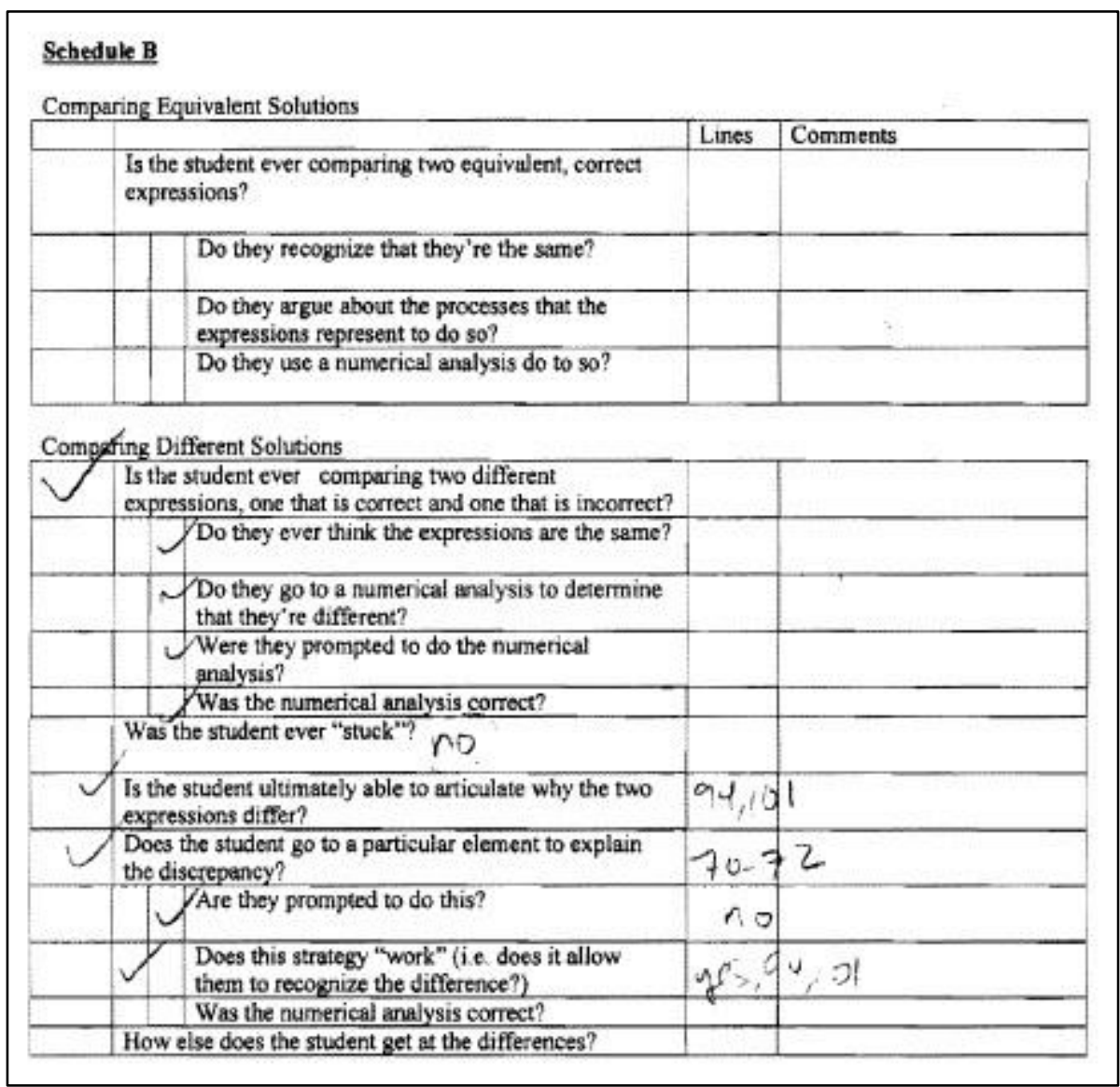

Figure 16

His language in Lines 70-72 below shows that he decided to appeal to a "specific outcome," which I took as evidence of an appeal to a particular outcome.

B: Um, then maybe there are, oh wait a second, let's say I assign, I think, yeah I think there's ways in which the order in which you choose the groups will end up with, it's like if I say I'm going to a specific outcome again... Let's say I choose group 1 as my first one, and, I'll use this paper, I choose group one as my first and I get GCDEA as my, as my group 1 .

In Schedule C, I coded Brandon's coordination of sets and processes. 


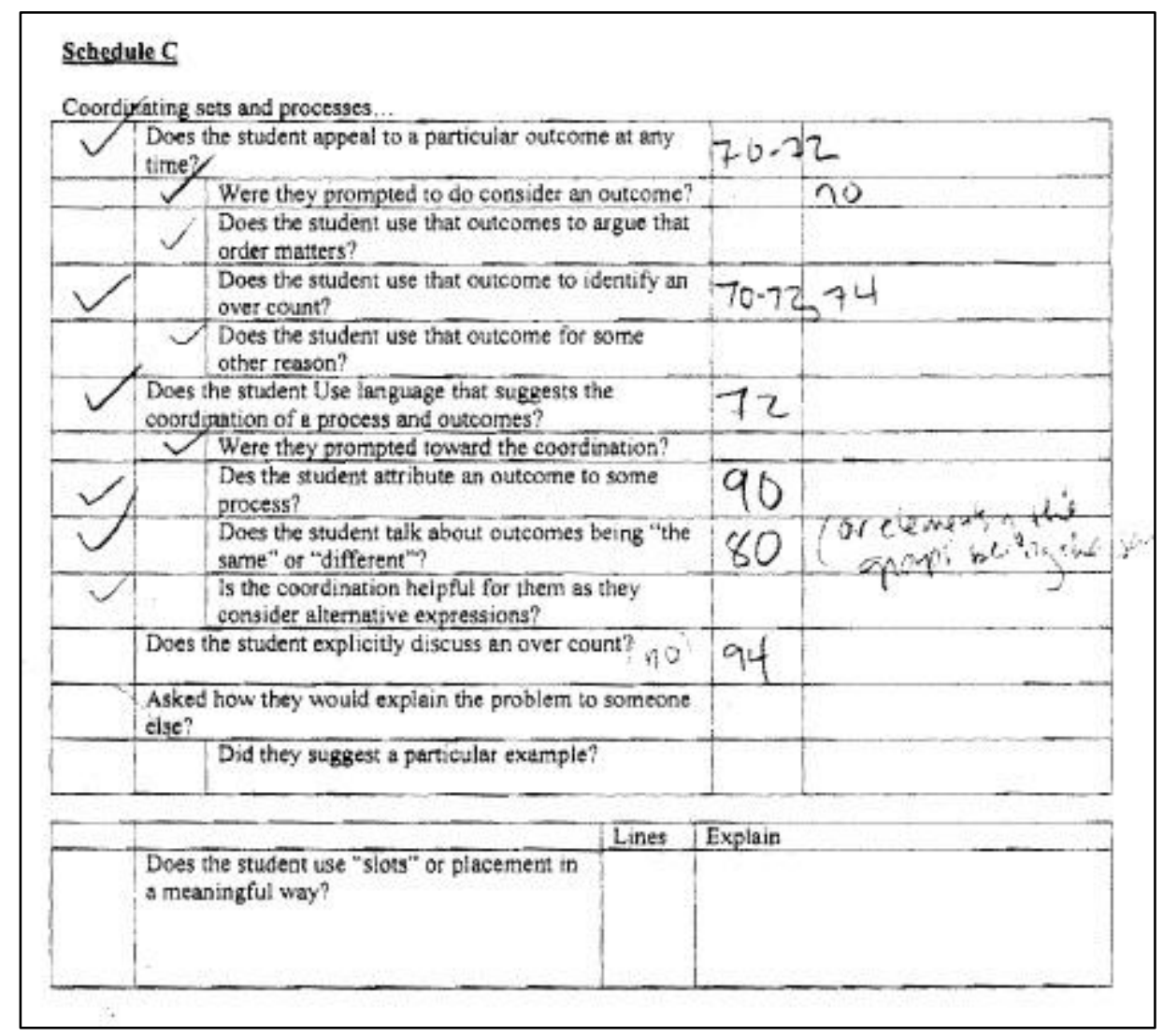

Figure 17

I cited Lines 70-72, shown above in Figure 17, as related to particular outcomes, and I note these lines along with line 74 (shown below) as evidence of Brandon referring to an overcount. Line 80 shows Brandon talking about language about outcomes being "the same thing."

Line 74:

B: Um, it seems to me that you could choose group 4 first, have GCDEA, so GCDEA is still together in one group, but it's not in group 1 anymore, it's in group 4. But it doesn't really matter, because the groups are just, they're just partitions of the whole, it's that like, there's nothing specific that group 4 is, is maybe doing that group 1 isn't doing, like, say, let's say we do have this class, and I've got 20 students in it, and it's just, I've assigned, uh, a reading, it's an 
English class, and I assigned a reading last night.

Line 80:

B: So um, in this case, um, group 4 is doing the same thing as group 1, calling them group 1 or group 4 doesn't make it a separate outcome,

Finally, in Schedule D (Figure 18) I discussed his diagram, and include the diagram he drew (Figure 19) below. He drew the diagram during his discussion in lines 72-74 shown above.

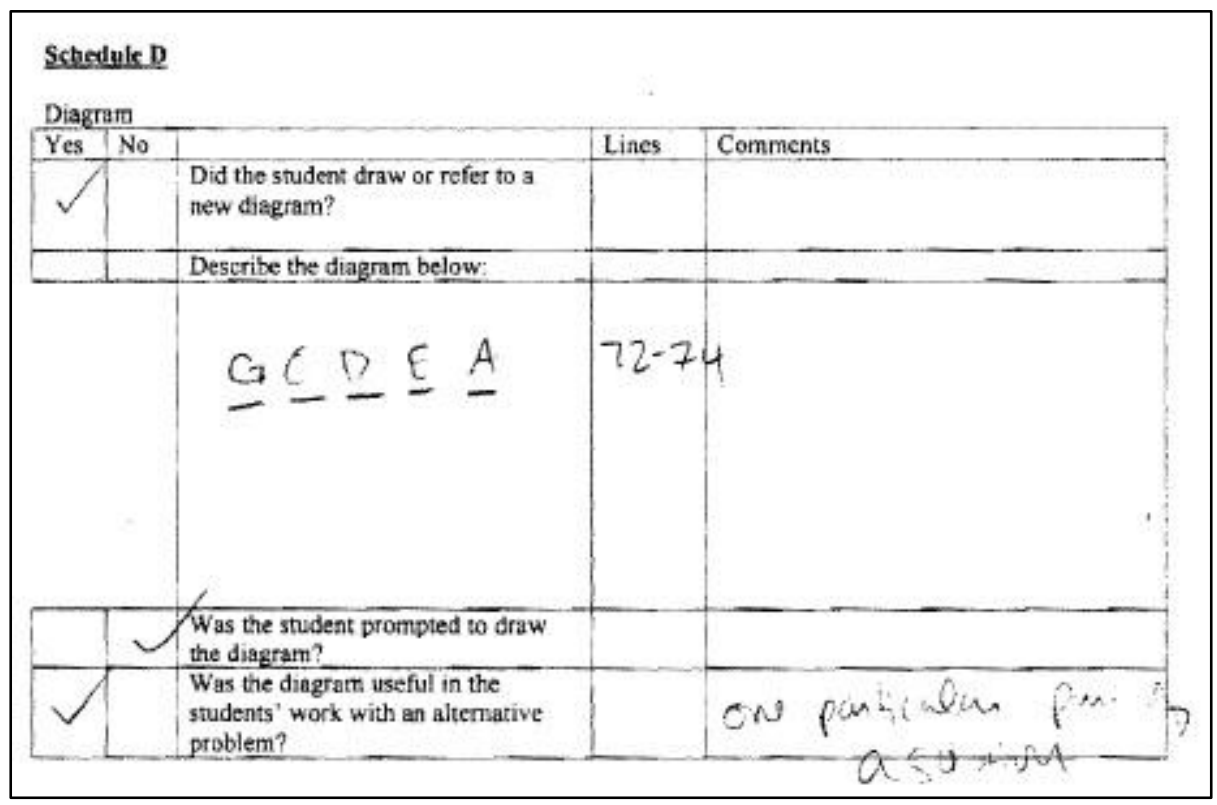

Figure 18 


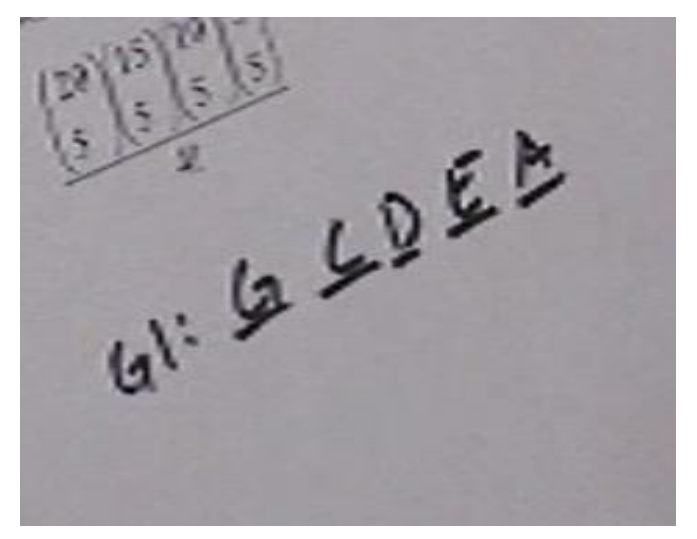

Figure 19

This description should thus give a sense of how I used Coding Scheme 2 to code the instances when students revisited problems. I went on to code all such instances, of which there were 70, using Coding Scheme 2.

3.5.4.3.4 - Analyzing the results from the Coding Schemes. After all of the schedules had been coded, I input them into an Excel spreadsheet (I had two separate spreadsheets for Coding Scheme 1 and 2, respectively). To do this I enumerated all of the boxes on the Coding Scheme, and I had each box correspond with a column in the spreadsheet. Each row of the column was a particular student's work on the problem, and I input $x$ 's into the spreadsheet in whatever corresponding boxes had $x$ 's in the Coding Scheme. This resulted in a large spreadsheet that I could manipulate, and I calculated some tallies of phenomena that related to set-oriented thinking. I was able to count the total number of times in which students used case breakdowns, for example, and to find the number of times in which students appealed to a particular element. I then went back through the Results chapter and used some of these tallies to give some context to the episodes, and these tallies were also incorporated into the Conclusions chapter. That is, I used the tallies 
to give some sense of whether a particular episode that I highlighted had occurred frequently or was relatively unique.

I do not consider these quantitative tallies to be the major results of my work. Rather, the themes related to set-oriented thinking that emerged from my grounded theory analysis are, in my view, the more substantive results. And it was these that contributed to the findings in the Results and the Conclusions chapters. However, the quantitative information has allowed me to elaborate the context in which many of the set-oriented instances occurred, and it has also enabled me to validate some of my interpretations of students' mathematical activity related to set-oriented thinking. Issues of validity and reliability related to my methods are discussed subsequently.

\subsubsection{4 - Step 4: Drawing upon the results from Steps 3 and 4 in order to draw}

conclusions. Finally, having written the Results chapter and also having coded the data with an eye toward identifying instances of set-oriented thinking, I went about drawing conclusions from the analysis of my data. To do this, I drew upon the themes that had emerged from the Results chapter and I also considered the results from having coded the schedules. In addition, the act of writing my findings and my conclusions helped in my refinement of the themes I had seen. The process of synthesizing the results contributed to my final description of the model and categories presented in the conclusions.

This process of writing the conclusions is related to the development of theory that both Auerbach and Silverstein (2003) and Strauss and Corbin (1998) describe. In particular, in writing the conclusions I connected the elements that I had been formulating from axial coding. The two final steps in Auerbach and Silverstein's stages for 
developing theory from raw text are: 5) Develop theoretical constructs by grouping themes into more abstract concepts consistent with your theoretical framework, and 6) Create a theoretical narrative by retelling the participant's story in terms of the theoretical constructs (p. 43). I did both of these things as I brought together ideas and themes in the conclusions of my study. Additionally, the drawing of conclusions involves the selective coding that Strauss and Corbin (1998) describe. They define selective coding as "the process of integrating and refining theory" (p. 143). In this type of coding (and this stage in the process from raw text to theory), the researcher now formulates theory by describing connections and relationships between the categories that came out of their axial coding. This involves more than just a listing of interesting phenomena or ideas that came out of the data; it also involves careful thought as to the structure of such themes. As Strauss and Corbin state, "if theory building is indeed the goal of the research project, then findings should be presented as a set of interrelated concepts, not just a listing of themes" (p. 145). As my Results chapter shows, a model that has emerged as a major conclusion goes beyond a list of themes, but I focus on the relationships between themes as well. Strauss and Corbin also encourage the use of diagrams in this final process of developing theory, noting that "diagramming is helpful because it enables the analyst to gain distance from the data, forcing him or her to work with concepts rather than with details of data" (p. 153). I drew upon both Auerbach and Silverstein's and Strauss and Corbin's works as I developed the results and conclusions of my study.

Additionally, as I developed the theory and wrote the Conclusions chapter, I drew upon episodes that I had written about and analyzed in the Results chapter. I included a 
number of illustrative examples from the data in my conclusions, and I also used some of the tallies that emerged from the Coding Scheme in order to contextualize episodes and findings that I mention in the Conclusions chapter. This concludes my description of my analysis process, which I described in four steps. Having described the analysis of my data in detail, I now address issues of validity and reliability, particularly related to the Coding Schemes.

\subsection{5 - Validity and reliability in the analysis}

The Coding Scheme gave me the opportunity to address issues related to validity and reliability in my study. I will address each of these below and will describe measures I took in an attempt to establish validity and reliability in my analysis.

3.5.5.1 - Validity. Qualitative studies are fundamentally different than quantitative studies in many ways, and as a result, it is reasonable that such studies involve different measurements of validity. Maxwell (2002) addresses the difficulty of dealing with validity in qualitative studies, and he suggests five different types of validity that concern qualitative researchers: descriptive validity, interpretive validity, theoretical validity, generalizability, and evaluative validity (p. 43). He does not claim that this is a complete list, nor that every consideration of validity fits nicely into one of these categories, but he proposes the list as a useful way to consider validity in qualitative studies. According to Maxwell, descriptive validity involves the accuracy of a qualitative researcher's account - the actual physical objects, events and behaviors involved in a particular study (p. 48). Interpretive validity goes a step further and has to do with researchers' interpretations of 
what these "objects, events, and behaviors mean to the people engaged in and with them" (p. 48, emphasis in original). In interpretive validity, Maxwell considers the participants' perspective, which may involve mental activity, cognition, affect, belief, and other such qualities. As in descriptive validity, accuracy is the focus of interpretive validity, and it is the elements of participants' perspectives that are determined to be accurate.

Theoretical validity is a third kind of validity that Maxwell (2002) describes. This kind of validity "goes beyond concrete description and interpretation and explicitly addresses the theoretical constructions that the researcher brings to, or develops during, the study" (p. 50). Theoretical validity "thus refers to an account's validity as a theory of some phenomenon" (p. 51, emphasis in original). Maxwell points out that theoretical validity can include issues that are not currently agreed upon within a research community. In discussing differences between descriptive/interpretive validity and theoretical validity, he says

My distinction between the two types is based on...the presence or absence of agreement within the community of inquirers about the descriptive or interpretive terms used. Any challenge to the meaning of the terms, or the appropriateness of their application to a given phenomenon, shifts the validity issue from descriptive or interpretive to theoretical (p. 52).

Theoretical validity involves establishing not only the accuracy of, but also the appropriateness of, the use or categorization of emerging theoretical concepts. Maxwell's (2002) remaining two types of validity are generalizability (which has to do with the extent to which a given account can be extended to other situations, p. 52), and evaluative validity (which arises when researchers make value judgments about an incident in a given account); these do not directly pertain to my study. 
I now use Maxwell's (2002) categorization of validity in qualitative studies to discuss validity in the study I conducted. I contend that I drew upon aspects of both theoretical and interpretive validity; in the paragraphs that follow I describe measures I took to consider validity, and I make a case for how my methods reflected these two types of validity. Because I used principles of grounded theory to develop themes from the raw data, a potential concern was that I might be relying too heavily on my own interpretation of what was happening in the data. Since I had personally spent so much time thinking about notions of set-oriented thinking, and because I had spent numerous hours engaging with the data, I wanted to, in some way, try to show that my ideas of set-oriented thinking were valid. That is, I wanted to be sure that my own interpretation about the kinds of student mathematical thinking in which I was interested (specifically ways in which students use set-oriented thinking) was legitimate. While the Coding Schemes described above (and in Appendix B) certainly served the purpose of allowing me to contextualize some of my results, they also played a role in establishing validity.

I now outline the specific ways in which I used the Coding Schemes to try to ensure that my study was theoretically valid; specifically I describe the development of the Coding Schemes, in which two other colleagues gave their feedback as I constructed the instrument. I made an initial attempt at writing Coding Scheme 1, which included writing a number of schedules that hit upon some of the ways in which I had seen set-oriented thinking arise for students. After I created a first version, I used it to code two interviews, 
making notes of changes I should make. I also had a colleague, Philip, ${ }^{23}$ try the first version on a problem I had coded and give me feedback, which led to further revision. Then, once I had a revised version of Coding Scheme 1, I wanted to compare answers and also make any further changes that would improve it.

Brian and Philip were both willing to help with double coding. There were 103 instances in which students attempted the problem initially (22 students attempting up to 5 problems each, but there were a handful of students who did not try all 5 problems due to time). I randomly chose 10 of these instances, using a random number generator, which would be coded by more than one person. Of the 10 particular instances I had chosen, I picked three of them at random that we would all code (Matthew's Groups of Students problem, Jenny's Cards problem, and Paige's Groups of Students problem). I instructed Brian and Philip on how to use Coding Scheme 1, by talking them through one interview I had previously done, and we all went on to independently code the three interviews I had randomly chosen. The three of us each used a second version of Coding Scheme 1, and we talked through the three problems and discussed the codes that everyone had given. We took time to talk about discrepancies that arose among our codes, and this provided an opportunity for me to better to communicate some ideas to the other coders about what I meant by some of the language in Coding Scheme 1. There was some discussion about what I meant by the coordination of sets and processes, and I

\footnotetext{
${ }^{23}$ Two colleagues helped me in establishing reliability in the coding process. Brian is a mathematics education PhD student, and Philip is a combinatorialist in the mathematics department (the names are pseudonyms).
} 
explained more carefully what I intended this idea to mean. The discussion of the details of the Coding Scheme was particularly meaningful and beneficial after they had done some coding (more so than the training session had been), and we were able to strengthen the instrument significantly through this discussion.

Philip and Brian suggested a number of helpful revisions to the Coding Scheme schedules. Specifically, in a number of cases, words like "mentioned" were changed to "used or mentioned," so the coder could code some activity if the student used, but did not make an utterance about, a particular idea. Also instead of having a separate "Smaller Case" Schedule, we decided to incorporate a box to check on each of the other schedules, indicating whether the particular activity was done in a smaller case. And, instead of having lists of set-oriented words on each schedule, it was suggested that we place one list of words on the front page. I also made a separate Diagram Schedule so that the coders could more easily code multiple diagrams. These specific changes to Coding Scheme 1, as well as a detailed discussion about the actual codes from some of these interviews, were quite beneficial. As a result, I made the adjustments and produced a new Coding Scheme 1 that was used for all subsequent coding of students' first attempts at the problems.

The fact that others were involved in the development of the Coding Schemes was one step toward validating my work. By getting other knowledgeable individuals' views and opinions on the development of the instrument, I was able to articulate my thinking to them and get constructive feedback about my understanding of ways in which students used of set-oriented thinking. Involving others essentially opened up the whole process 
and hopefully kept me from becoming too myopic in my thinking about the issues at hand. I contend that the activity of having other colleagues my work was an attempt at establishing theoretical validity. When we all coded some episodes, and then met to discuss them, I was getting their feedback on phenomena that were not strictly coming from my participants' perspectives. We coded for (and went on to discuss and refine) some phenomena that involved some aspect of theory that I was developing; they could not be checked for accuracy by looking back at the videotape. For example, some coding involved questions about "referring to a particular outcome," or to "coordinating sets and processes." These were constructs that had emerged from my work, and they do not exist as known constructs in the research community. By having others weigh in on the development on an instrument that dealt with emerging ideas and constructs that I was formulating, I was addressing theoretical validity.

After the Coding Schemes were made, I distributed the remaining 7 episodes that had been randomly chosen among Philip, Brian, and me. Brian also coded an additional 8 episodes, and I coded the remaining 155 episodes. The double coding that Philip and Brian helped with (which is described in more detail in Appendix B) was done for the sake of interpretive validity (and reliability, as mentioned below). That is, by having Philip and Brian also examine and code students' mathematical activity (particularly related to set-oriented thinking) I could compare their results with mine and see the extent to which our codes matched. If I could establish that our codes matched to a certain degree, this could suggest that my own interpretation of the students' mathematical activity was not somehow occurring in a vacuum. I maintain that this particular aspect of 
the study has to do with establishing interpretive validity. It is interpretive and not descriptive because we coded phenomena that had originated from the participants' perspective, and we did not simply code externally observable objects, behaviors or events that were free of interpretation or meaning. It is interpretive and not strictly theoretical because after Philip, Brian, and I met and refined the Coding Scheme (and clarified and discussed some of the theoretical constructs that I had developed and had included in the Coding Scheme), the phenomena that we were coding became agreedupon phenomena among the three of us. Thus the coding they did addressed the issue of how accurately we identified particular phenomena. By having Philip and Brian double code, I was establishing accuracy, and Maxwell (2002) would describe such activity as addressing interpretive validity.

In sum, I have made attempts at addressing both theoretical and interpretive validity at different points in my analysis of data. By getting external input on the development of the instrument, I attempted to establish theoretical validity. Doing so ensured that the constructs I was developing as elements of the Coding Scheme were not based too heavily on my own understanding alone. Then, by having external coders also code a number of episodes, I addressed interpretive validity by attending to the accuracy of the coding of such phenomena.

3.5.5.2 - Reliability. Because I ended up doing a majority of the coding (I coded a total of 95 of the 103, and I coded all 70 of the incidents when students revisited the problem), there was not as much of a need for me to establish inter-rater reliability. That is, if I had divided up the codes more evenly among the coders, it would have been important to 
establish consistency among the coders; as it stands, however, I was able to do the vast majority of the coding, which reduced the need to measure such consistency. I do suspect that aspects of the development of the Coding Scheme, and particularly my interaction with other coders, did have an impact on my own personal consistency with the instrument. More specifically, the activity of training other coders and receiving feedback from them about the instrument likely caused me to be more precise and consistent in my own coding.

Nonetheless, I did compute some measure of inter-rater reliability on those Coding Schemes that Philip, Brian, and I coded. In particular, I tallied the number of boxes on each schedule, took a raw score of how many boxes matched and then calculated percentages that indicated the extent to which we agreed. I found that the three problems that Brian, Philip and I coded prior to the refinement of Coding Scheme 1 each matched in the range of $80 \%-88 \%$, and after the refinement of the instrument, the rest of the reliability measures were above $87 \%$ (with one exception which matched $80 \%$ ). More numerical data, as well as details about the computation, can be found in Appendix B.

These measures above give a sense of the reliability of the instrument. In particular these numbers show that I was supported in my own understanding of the mathematics and the ways in which some of the phenomena related to set-oriented thinking might have been showing up in the interviews. Of course I was (and am) more familiar with my data than the other coders were, but by establishing some measure of agreement in our coding, I was able to lend credence to my own coding of the data. 
One other point to make about validity and reliability is that I decided only to have double coders on students' initial work on the problem. While I ultimately coded both students' original work and the times in which they revisited the problems, Philip and Brian only coded episodes using Coding Scheme 1. This was primarily for efficiency's sake, but it is justifiable in terms of validity and reliability. The Coding Schemes have to do with my interpretation of the students' mathematics, particularly how I interpret students' work involving sets of outcomes, and I felt that I could test the validity of my interpretation of set-oriented thinking on the first treatment of the problem alone. Since the students' mathematical activity that I was analyzing (and particularly the determination of whether or not students were engaging in some of the activities that I took as set-oriented thinking) was similar in both original and revisiting situations, having other coders examine students' first treatment of the problem could ensure that my own thinking about set-oriented thinking was valid. That is, I did not need to check for validity again using Coding Scheme 2. In addition, since I knew I was going to be the only coder of those instances in which students revisited problems, testing for inter-rater reliability was not essential.

\section{6 - Summary of the Methodology chapter}

In this chapter, I provided the details of the implementation of the study, including the specific research methodologies I used. I began with addressing the ways in which the pilot study shaped some of my design decisions. I then proceeded to describe how I designed and carried out the study, beginning with a description of the methodological 
framework of grounded theory. I then provided specifics of data collection, including a description of and a rationale for the interview tasks. I went on to elaborate details of the data analysis, which took place in several stages and which was influenced by existing methodological frameworks. This Methodology chapter has filled out the methodological details of what I actually did to gather and analyze the data, and it should provide a context for what I did in order to generate my results and ultimately synthesize those results into theoretical contributions. 


\section{Chapter 4 - Results}

The Results chapter consists of two major parts. First, I present as a result a model of students' counting activity. This model is both an outcome of my analysis and a lens through which I analyzed the data. I consider it to be a conceptual analysis of students' counting activity, but one that has been refined and elaborated through the process of analyzing data ${ }^{24}$. Therefore, the model is both an outcome of my study (indeed, no other model for students' counting activity currently exists), and also it played an important role in my data analysis.

Second, in this Results chapter I provide a narrative of what occurred in the interviews. Specifically, for each of the five problems that students attempted in their interviews, I offer selective details about some of the students' work, and I summarize other relevant information more broadly. By doing so, I hope to provide the reader with a sense of the variety of student approaches to the respective problems to further illuminate some mathematical subtleties of the problems. My goal in this second part of the chapter is to provide the reader with a sense of what the interviews entailed, and to provide a backdrop of some of the ways in which students drew upon (or did not draw upon) set-

\footnotetext{
${ }^{24}$ I present the model here and not earlier as a theoretical perspective because it was a result that emerged from my study. That is, while many of the mathematical ideas that exist in the model emerged before I analyzed data (and while the model itself was a tool by which I analyzed data), the model presented below was also greatly refined during that analysis process. Specifically, the mathematical ideas and relationships in the model were refined as I used it in my data analysis, and what resulted was an even more well-defined and articulated version of the model. So the model was developed primarily as a conceptual analysis, from mathematical concepts, and it was not developed from the data. However, it was not developed completely independently either; rather, the data helped to flesh out and refine the model.
} 
oriented thinking as they solved counting problems. In order to organize this chapter efficiently, I will discuss each problem individually. I will provide greater detail for this section in Section 4.2 below.

4.1 - A model of students' counting activity

4.2 - Episodes of student work, organized by problem

4.3 - Overall summary of the results chapter

\section{1 - A model of students' counting activity}

In this section I propose a model (Figure 20) that further explains this coordination of sets and processes in combinatorial problem solving, highlighting relationships between counting formulas/expressions, counting processes, and sets of outcomes. In what follows, explicate the components of the model and describe the ways in which these different components interact with each other. Throughout the chapter I connect my findings and conclusions to this model.

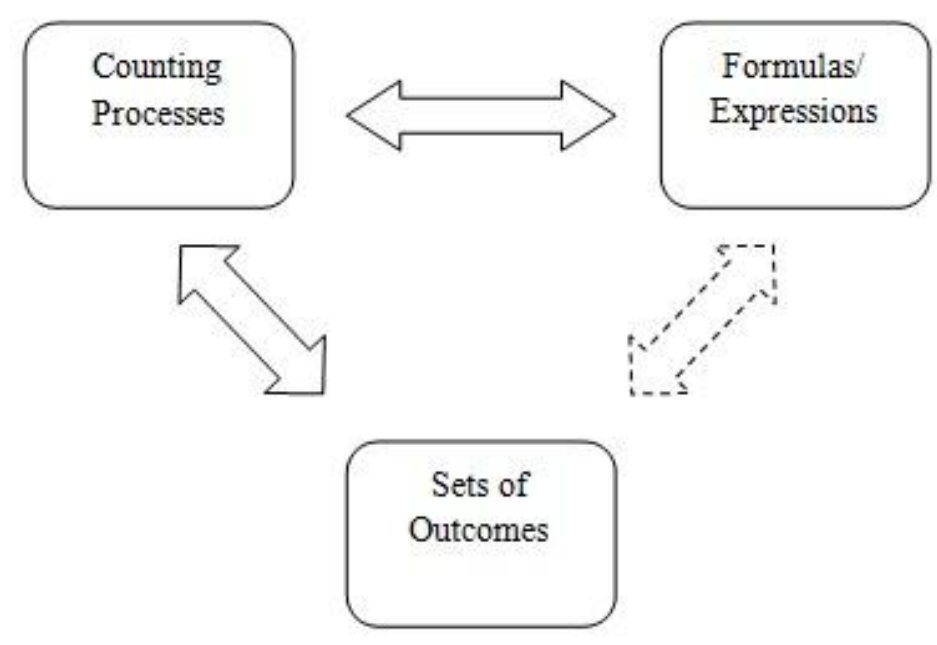

Figure 20 
I begin by explaining each of the components of the model: Formulas/Expressions, Counting Processes, and Sets of Outcomes. By Formulas/Expressions I mean mathematical expressions that yield some numerical value. The formula could have some inherent combinatorial meaning (such as a binomial coefficient $\left(\begin{array}{l}8 \\ 3\end{array}\right)$ ), or it could be some combination of numerical operations ${ }^{25}$ (such as a sum of products $9 \cdot 13+3 \cdot 12$ ). By Counting Processes, I mean the enumeration process (or series of processes) in which a counter engages (either mentally or physically) as they solve a counting problem. By Sets of Outcomes I mean those sets of elements that one can imagine being generated or enumerated by a counting process. In the context of a counting problem, this may be the set whose cardinality represents the answer to that counting problem, but sets of outcomes could also refer to any set that can be associated with a counting process (even if that set is not the answer to the counting problem at hand). For a given counting problem, a student may work with one or more of these components and may explicitly or implicitly coordinate them. I now elaborate the key relationships between these components.

\subsection{1 - Key relationships between components of the model}

\subsubsection{1 - Counting processes and expressions/formulas}

\footnotetext{
${ }^{25}$ While my study uses a relatively restricted definition of formulas/expressions, this category could potentially be extended to include other combinatorial tools and techniques. This idea is discussed later as an avenue of further research.
} 
In order to most effectively discuss the model, I first discuss the relationship between a counting process and an expression or formula, which addresses the relationship highlighted below in Figure 21.

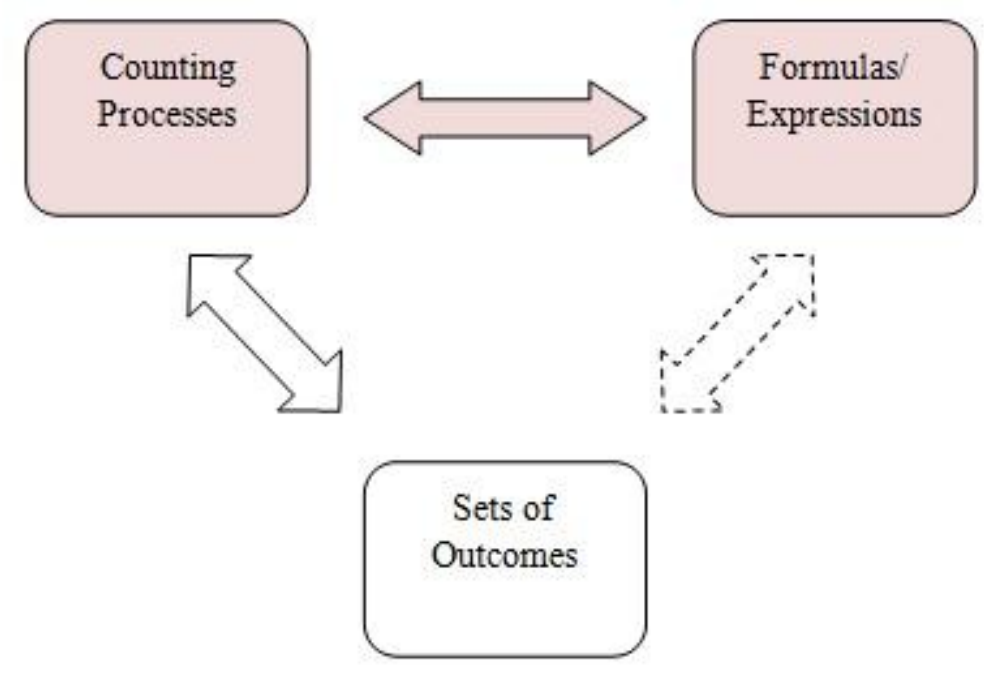

Figure 21

The relationship between counting processes and formulas/expressions is not trivial, and it is an important aspect of understanding what is involved in solving a counting problem. I propose that in the context of a counting problem, a given mathematical expression can often naturally be associated with a counting process. For example, we may consider an expression like $\left(\begin{array}{l}5 \\ 2\end{array}\right) \cdot\left(\begin{array}{l}5 \\ 3\end{array}\right)$. This product of binomial coefficients can represent a number of things. From one perspective, it is a just number; we could calculate the product to arrive at 100 . However, in the context of counting, this same product typically signifies a particular process - specifically, it is an instance of the multiplication principle. A typical element of the set of outcomes is constructed in two 
stages; in the first, two objects are chosen from five distinct objects, and in the second three objects are chosen from five distinct objects. The multiplication indicates that the two stages are chosen independently ${ }^{26}$. We can further specify a context, such as the Test Questions problem, and suddenly the expression can represent an even more specific process - choosing two of the first five questions and then choosing three of the second five questions. In another context, this process might involve choosing committee members or books. Regardless of the context, however, counters can attribute combinatorial meaning to a mathematical expression in the form of an enumeration process.

In the opposite direction, we could conceptualize a counting process that generates an appropriate formula. If we wanted to count the number of ways of arranging 5 objects from a set of 10 distinct objects, there is a counting process that would allow us to do that, and this counting process could be expressed through a formula. We could consider the number of options for our first position (10), then consider the number of options for our second position (9), etc., and using the multiplication principle we could arrive at an answer of $10 \cdot 9 \cdot 8 \cdot 7 \cdot 6$. So there are formulas and mathematical expressions that can represent a particular counting process with which we want to engage. In fact, this particular act of generating a formula from a counting process is what "solving a counting

${ }^{26}$ In general, a binomial coefficient $\left(\begin{array}{l}n \\ k\end{array}\right)$ represents the number of ways of choosing $k$ objects from a set of $n$ distinct objects. There is thus an enumerative meaning to that expression, and the language of "choosing" does represent some number of outcomes that have been counted. 
problem" often entails. Also, in counting problems with very large numerical answer, often an expression or a formula can be just as meaningful as a numerical value.

Now, there may be more than one counting process associated with a formula, and as an example we consider the expression $\left(\begin{array}{l}10 \\ 5\end{array}\right)$. This is a numerical expression (algebraically it is equivalent to $\frac{10 !}{5 ! 5 !}$, or 252 ). If we consider the questions "How many ways are there to choose a committee of 5 people from a faculty of size 10 ?" The answer to this question is $\left(\begin{array}{l}10 \\ 5\end{array}\right)=252$, but there are two different counting processes that could get us there, each represented by the same expression of $\left(\begin{array}{l}10 \\ 5\end{array}\right)$. We could first have arrived at the answer by choosing 5 of 10 people to be in the committee, yielding $\left(\begin{array}{l}10 \\ 5\end{array}\right)$. We also could have arrived at the answer by choosing 5 people not to be on the committee, also done in $\left(\begin{array}{c}10 \\ 5\end{array}\right)$ ways. So, while the expressions are the same, the processes that arrived at the expressions differ. The appropriate counting process would depend on a student's way of thinking about the problem.

It also may be the case that there may be more than one different (though mathematically equivalent) expression associated with some counting process. An example is that two different students may have memorized different expressions for the process of choosing a set of $k$ objects from $n$ distinct objects. For one student, an 
expression associated with that process may be $\left(\begin{array}{l}n \\ k\end{array}\right)$, for another it may be $\frac{n !}{(n-k) ! k !}$.

While these are mathematically equivalent (in the sense that they can simplify to the same expression) and I take them to be different expressions, they may be associated with the same counting process ${ }^{27}$.

There also may be multiple processes that arrive at mathematically equivalent expressions, although if the processes differ the forms of the expressions tend to differ. For example, if we wanted to arrange 5 objects in 10 slots, we could use the multiplication principle to successively place objects in positions, arriving at $109 \cdot 8 \cdot 7 \cdot 6$. However, instead of arranging 5 of 10 objects in slots, we could first choose 5 of the 10 objects, done in $\left(\begin{array}{l}10 \\ 5\end{array}\right)$ ways, and then arrange them in 5 ! ways. This yields an answer of $\left(\begin{array}{l}10 \\ 5\end{array}\right) \cdot 5$ !. The expressions $10 \cdot 9 \cdot 8 \cdot 7 \cdot 6$ and $\left(\begin{array}{l}10 \\ 5\end{array}\right) \cdot 5$ ! are equivalent but they differ in form, and the counting processes underlying them are different.

Also, I want to be clear that I am interested in students' construction of the relationship between counting processes and formulas/expressions (and vice versa). That is, regardless of whether we take a Platonic view that there is one, particular process that is fundamentally represented by some expression, what I am interested in is students' construction of this relationship. I care about students' construction of processes given

\footnotetext{
${ }^{27}$ I acknowledge that I cannot claim to know what process a student may associate with an expression, or vice versa. The discussion is meant to elaborate the relationship between formulas/expressions and counting processes.
} 
formulas (or of formulas, given processes) and not the objective reality of this relationship, if there is one.

Further elaboration of the relationship between counting processes and formulas/expressions - examples from the data. In order to further elaborate the model and to ground it in the results of my study, I offer two examples that highlight this relationship between counting processes and formulas/expressions. The first example shows a student for whom it was natural to associate a process behind a given combinatorial expression. The second example shows a student who was unable to construct such a relationship.

First, we consider part of Joshua's work on the Test Questions problem. He had initially gotten the problem right, yielding

$$
\left(\begin{array}{l}
5 \\
2
\end{array}\right) \cdot\left(\begin{array}{l}
5 \\
3
\end{array}\right)+\left(\begin{array}{l}
5 \\
3
\end{array}\right) \cdot\left(\begin{array}{l}
5 \\
2
\end{array}\right)+\left(\begin{array}{l}
5 \\
4
\end{array}\right) \cdot\left(\begin{array}{l}
5 \\
1
\end{array}\right)+\left(\begin{array}{l}
5 \\
5
\end{array}\right) \cdot\left(\begin{array}{l}
5 \\
0
\end{array}\right)
$$

and I asked him to evaluate the answer $\left(\begin{array}{l}5 \\ 2\end{array}\right) \cdot\left(\begin{array}{l}8 \\ 3\end{array}\right)$, which is too big. We see below that he analyzed the alternative answer and described a process that he associated with the expression.

$\mathrm{J}$ : Um, okay. Uh, yeah, this $\left[\left(\begin{array}{l}5 \\ 2\end{array}\right) \cdot\left(\begin{array}{l}8 \\ 3\end{array}\right)\right], \ldots$ what you have done is like, you picked up 2 questions from the first, uh, 5 questions, from the rest of the 8 questions, which are left out, you picked up 3 of them. 
What I want to highlight in this example is that Joshua seemed to have talked fairly easily about the fact that the numerical expression in front of him, $\left(\begin{array}{l}5 \\ 2\end{array}\right)\left(\begin{array}{l}8 \\ 3\end{array}\right)$, represented a counting process. Although I presented him with only a product of binomial coefficients (and not an explanation of a counting process), he realized that it represented picking 2 of the first 5 questions and then 3 of the 8 remaining. Throughout the interviews, for the most part, students naturally made that connection between counting processes and formulas; in fact it appeared to be so engrained that it rarely came up as something they felt they had to discuss or explain.

Our second example provides one exception, however, as the relationship between an expression and a counting process was not so clearly evident for one student. Nancy had worked on the Apples and Oranges problem and had eventually counted up cases correctly to arrive at the answer of 53. In the excerpt below, I had given her an alternative answer of $6 \cdot 9-1$.

$\mathrm{N}$ : 6 times 9 minus 1. Yeah I don't know what that is. Does mine turn out to 53, I don't know.

E: I think yours would turn out to 53 .

$\mathrm{N}$ : Why do that? Yeah I don't know where this is coming from. I have - I don't know how they did that.

E: Okay. What if I asked you to just take another minute or two and think about it...

N: Okay. Well it's, it's 5 plus 1 times 8 plus 1 minus 1 . So I don't know if that works all the time or not.

E: $\quad$ Okay so where could the 5 plus 1 times 8 plus 1 come from?

$\mathrm{N}$ : Well 5 apples and 8 oranges, so if that's a formula that works,

E: Okay, and how about, why does that make sense in terms of the problem. Like, what - why would 5, like why multiplication...

$\mathrm{N}$ : I don't know, I don't know. 
In this example Nancy could not see why $6 \cdot 9-1$ could be a correct answer. Even more, though, there is nothing in her language above that suggests that she tried to connect the expression to some underlying counting process. I suspect that the new expression was so different from what she had done that she perhaps did not even know where to begin, or perhaps she was tired of doing counting problems, but I still find it noteworthy that there was no attempt to try to determine the reasonability of the formula by connecting it to a counting process. As I mentioned, this example was quite exceptional.

In this section I have described the relationship between counting processes and formulas/expressions, providing both mathematical explanation and also using examples from the data to highlight the relationship. I now turn to discussing the relationship between counting processes and sets of outcomes.

\subsubsection{2 -Counting processes and sets of outcomes}

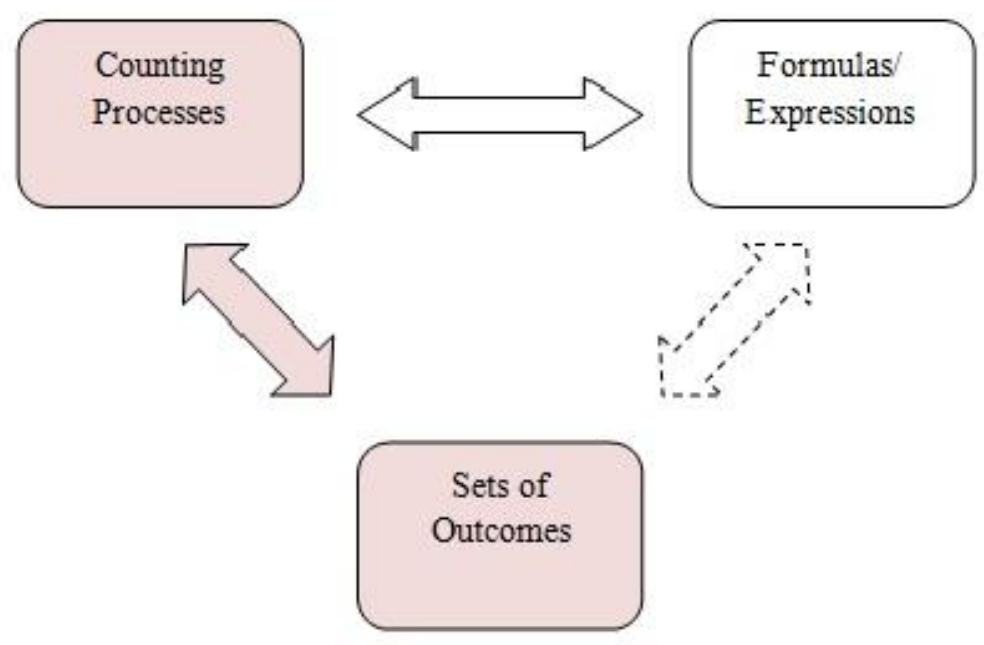

Figure 22 
As with the relationships between counting processes and formulas/expressions, the relationship highlighted in Figure 22 above is bi-directional. On the one hand, counting processes may generate some set of outcomes. Conversely, a given set of outcomes may be enumerated (or its size may be determined) via some counting process. I have addressed this to some extent in the Literature Review chapter, but here I provide further evidence of the importance of this coordination. For a given counting problem, the answer to the problem may be conceptualized as a counting process that arrives at an appropriate expression, but it also may be conceptualized as the cardinality of an appropriate set of outcomes. Students may recognize that the answer to the problem can be found by applying an appropriate counting process (there may be more than one), and/or they could recognize that the answer to the problem can be found by determining the cardinality of the set of outcomes. Answering a counting problem can be related to both of these components of the model.

I elaborate the following example to delineate the relationship between counting processes and sets of outcomes: "How many 3-letter 'words' are there using the letters A, B, and C (repetition allowed)?" The set of outcomes associated with that problem are the three letter words that satisfy the constraint, of which there are 27 . There are multiple counting processes that could correctly answer the counting problem, and I discuss two such processes for this example. First, the question can be solved in a straightforward manner using the multiplication principle; one possible counting process is first to apply the multiplication principle to consider the number of choices for the first letter, second letter, and third letter. The choices are independent, and, per our discussion above, this 
process can be represented by the expression $3 \cdot 3 \cdot 3=27$ solutions. A second process that would solve the problem is to break the problem into cases according to the number of repeated letters in a particular outcome. That is, we first consider the solution with all A's, all B's, or all C's, then we consider solutions consisting of just A's and Bs, just A's and C's, or just C's and B's, and then we consider solutions consisting of one of each letter. The three respective parts of the case breakdown are size 3, 18, and 6, respectively, which gives a total answer of $3+18+6=27$.

The relationship between the counting processes and the set of outcomes can be conceptualized in a couple of ways. On one hand, a counting process can be seen as actually generating some set of outcomes, and in fact different processes can result in different structuring of the set of outcomes. Staying with the example above, the process of considering choices for the three respective positions in the word produces a particular listing of the set of outcomes. That is, by considering first that the first letter can be A, B, or $\mathrm{C}$, and then noting that for each of those choices, the second letter can be $\mathrm{A}, \mathrm{B}$, or $\mathrm{C}$, and so on, the set of outcomes can be generated in Figure 23 as follows. The tree diagram in Figure 23 can be useful in making the generation of outcomes more apparent; the structure of the diagram gets at the 3-stage process of the multiplication principle, and to the right we see the listing of the set of outcomes. 


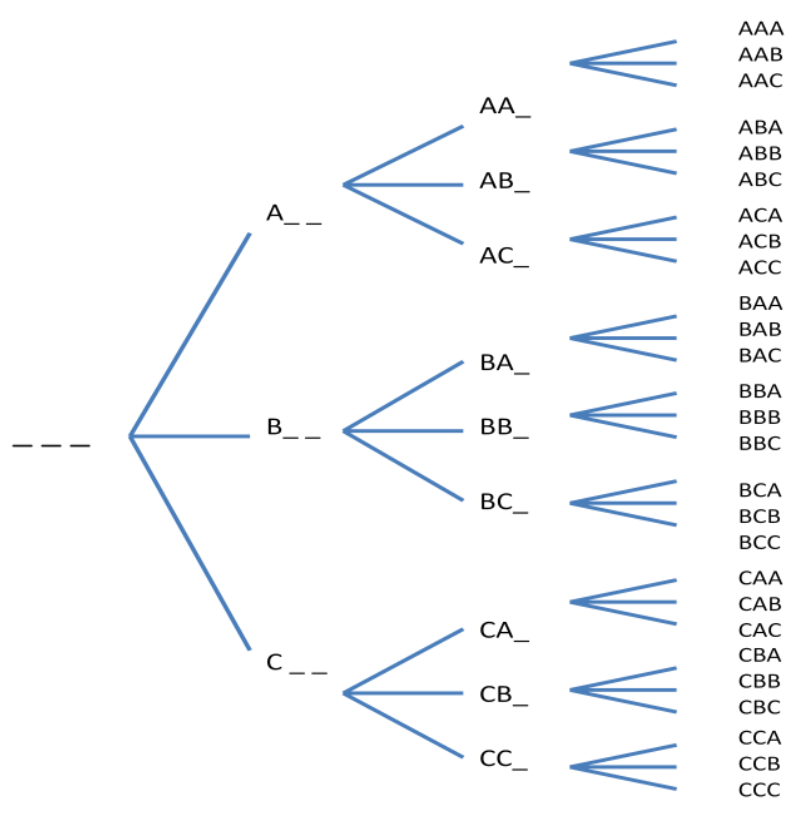

Figure 23

In addition to generating a set of outcomes, a counting process can impose a structure onto set of outcomes. We see in Figure 23 that the counting process in the tree diagram actually organizes the set of outcomes into an alphabetical list, and, given our process of considering letter options for the respective positions, this makes sense. Alternatively, the process of breaking the problem into cases and counting words based on the number of repeated letters can be seen as organizing the set of outcomes in a different way. In Figure 24 below, I have included on the left the alphabetical list of outcomes that was generated by the multiplication principle process, and on the right the list of outcomes based on the number of repeated letters. The diagram below shows the two ways in which the different counting methods structured the set of solutions; there is a one-to-one correspondence between the set on the left and the set on the right. The set of outcomes is represented in both lists, and the cardinalities are the same, but the processes that yielded 
the set of outcomes differed. This example of two different counting processes illustrates the fact that a counting process imposes a structure on the set of objects being counted.

\begin{tabular}{|c|c|c|c|c|c|c|}
\hline & & & AAA & \multicolumn{2}{|l|}{ BBB } & $\mathrm{CCC}$ \\
\hline AAA & $A A B$ & AAC & & & & \\
\hline ABA & $A B B$ & $A B C$ & $A A B$ & ABA & & BAA \\
\hline ACA & ACB & $A C C$ & BBA & BAB & & $A B B$ \\
\hline & & & $A A C$ & $A C A$ & & CAA \\
\hline & & & CCA & CAC & & $A C C$ \\
\hline BAA & BAB & BAC & & & & \\
\hline BBA & BBB & BBC & $B B C$ & BCB & & CBB \\
\hline BCA & $\mathrm{BCB}$ & $\mathrm{BCC}$ & CCB & $\mathrm{CBC}$ & & $\mathrm{BCC}$ \\
\hline CAA & CAB & CAC & & & ACB & \\
\hline CBA & CBB & $\mathrm{CBC}$ & & & BCA & \\
\hline CCA & CCB & $\mathrm{CCC}$ & & & CBA & \\
\hline
\end{tabular}

Figure 24

The discussion above has focused on one direction of the relationship - how a student can generate (and organize) a set of outcomes from some counting process. I now discuss the other direction, in which a student can arrive at a counting process from a set of outcomes. For both of the processes described above we could also think about starting with the set of outcomes, deciding to organize it in a particular way, and then coming up with a formula to enumerate the set that is consistent with that organization of the set. For instance, we could have decided to start the problem by imagining (or actually) listing the outcomes alphabetically. This could have led to the consideration of choices for each 
letter, and ultimately to the multiplication principle. Or, we could have decided that we wanted to break the outcomes up according to the number of different letters in each word, and then we could have determined how many passwords were in each possibility. In fact, I contend that case breakdowns are an illustrative example of this direction of the relationship between sets of outcomes and counting processes (indeed, cases often arose in my study in this way). A student can determine that they want to structure the set of outcomes into cases based on some constraint (regardless of whether they can conceptualize every element of the set, which I will further discuss below), and that is going to determine how they go about actually selecting an appropriate counting process.

Finally, there could be a back and forth relationship between counting processes and sets of outcomes. That is, a person could start a counting problem by choosing a particular counting process, and they could consider the set of outcomes that it generates. In a case in which those outcomes the process generated do not align with the desirable set of outcomes, the person might compare those sets of answers and then try to engineer a counting process that correctly enumerates the desirable set of outcomes. I thus contend that the relationship between counting processes and sets of outcomes can be (and should be) a very flexible, fluid relationship, in which counters easily move from one component to another. If the counter can easily coordinate a counting process and a set of outcomes, this affords them much tractability in their counting. Indeed, there are often multiple ways to solve a counting problem, and such approaches can amount to different ways of organizing the set of solutions. In this sense, counting can be seen as an activity that relates counting processes to an underlying set of outcomes. 
An important aspect of the set of outcomes is that while the set of outcomes may exist as the set whose cardinality is the answer to a counting problem, a student may not necessarily want to (or be able to) consider the whole set of outcomes. In some cases, the size of the set of outcomes might be quite manageable, and it might not be difficult to actually list out the set of outcomes or to somehow conceive of the entire set of outcomes. For sets that are bigger, though, students may not be able to list out all the solutions, and they may not even be able to conceive of the set all at once as some finite list of elements. It is possible to think about very large sets of outcomes, however, and even about organizing them, perhaps by thinking abstractly or by considering representative elements of the set of outcomes. So when we talk about students considering a set of outcomes and then thinking about what counting process might enumerate (or about the set of outcomes that some process generates), they may be thinking about the set abstractly or may be theoretically organizing the set of outcomes.

Finally, an important point to make about this relationship between counting processes and sets of outcomes is that a set of outcomes can provide a way for students to ground their combinatorial activity and ultimately determine whether a counting process is correct. Part of what makes counting fascinating is that counting processes can seem correct but can actually be wrong. Counting processes can be considered in isolation of the set of outcomes (students may focus solely on the relationships between counting processes and formulas discussed above), and students can arrive at answers to counting problems that are based on a counting process alone. While this counting process may generate a set of outcomes, it may not be the set of outcomes whose cardinality is the 
answer to a particular counting problem. There can thus be a disconnect between what the set of outcomes a given process is actually counting and the set of outcomes that is to be enumerated (the set whose cardinality is determines the answer to the counting problem). Students can determine whether a process is counting correctly by grounding their work in a set of outcomes, and by thinking about how the process can be used to structure the set of outcomes in some way that facilitates the enumeration of the set.

Further elaboration of the relationship between counting processes and sets of outcomes - examples from the data. The majority of my discussion in this conclusions chapter has to do with this relationship between counting processes and sets of outcomes. While I provide examples here to illustrate the discussion above, these ideas will be elaborated further in the remainder of the chapter.

As an example of the relationship between counting processes and sets of outcomes, we consider Makaena's work on the Groups of Students problem. Of particular note is her use of the "same" and "different" language. Makaena had arrived at the (incorrect) answer

$$
\left(\begin{array}{l}
20 \\
5
\end{array}\right) \cdot\left(\begin{array}{l}
15 \\
5
\end{array}\right) \cdot\left(\begin{array}{l}
10 \\
5
\end{array}\right) \cdot\left(\begin{array}{l}
5 \\
5
\end{array}\right)
$$

and we were in the process of discussing the (correct) answer

$$
\frac{\left(\begin{array}{l}
20 \\
5
\end{array}\right) \cdot\left(\begin{array}{l}
15 \\
5
\end{array}\right) \cdot\left(\begin{array}{l}
10 \\
5
\end{array}\right) \cdot\left(\begin{array}{l}
5 \\
5
\end{array}\right)}{4 !}
$$

I had asked Makaena how she might explain to someone else why the division by 4 factorial was necessary. She talked through an example and highlighted what would 
happen as people were chosen to be in different groups - that the same 5 people could have been chosen first or second, but that would not necessarily mean a different division of the 20 people.

E: So what if someone came to you and gave you, you know just the numerator there? How would you explain what, or, I mean same kind of deal, or...

M: Yeah I guess I would draw a picture. And say, okay well let's, um, you know imagine picking 5 people and putting them in this group, and then I'd actually list out either some names or some letters or something like that. And, um, okay, let's pick 5 out of the rest of the people, put those in here [one of the circles she drew]. And then have them imagine, um, saying okay, since in the beginning we could have picked, um, 5 from the whole group, what if it had been the 5 that were picked here in the second group, what if those [5 other people] were picked first? $\underline{\text { Right and then that second group was now it could have been any of those people }}$ from the first group, and let's say that was there. Is that a different set of four groups, or is it the same? And that kind of thing. So I think examples would be the most persuasive in that case.

In her underlined work I interpret her to be describing a counting process, and her question, "Is that a different set of four groups, or is it the same?" suggested to me that she considered that process to have generated an outcome of some set of four groups. As she explained her work, she considered a set of outcomes, and she realized that the problematic expression represented a process that actually generated some outcomes multiple times that should not have been counted as different. She was thus able to identify why an expression was incorrect ${ }^{28}$.

\footnotetext{
${ }^{28}$ This "same" and "different" language was used frequently throughout the interviews (30 times in students' original attempts at the problems, and 47 times when students revisited), and in fact when students were able to identify instances of overcounting, $80 \%$ of the time (32/40) they did so by appealing to two elements that were the same or different.
} 
We also recall Joshua's example on the Test Questions from above, in which he analyzed the incorrect expression $\left(\begin{array}{l}5 \\ 2\end{array}\right) \cdot\left(\begin{array}{l}8 \\ 3\end{array}\right)$. Joshua had correctly articulated a counting process by which the expression was represented, namely that it indicated first choosing 2 of the first 5 problems, and then from the remaining 8 problems, choosing 3 . This is a correct interpretation of what a process might be that yielded the expression. However, Joshua went on to state that he believed that expression to be equivalent to his correct expression, which was an incorrect assertion. Here Joshua's error was not because there was a mistake in the relationship between the expressions and the counting processes, but because the two counting processes were not actually the same. From my perspective, I knew the two processes were not the same because they did not count the same set of outcomes (the process behind $\left(\begin{array}{l}5 \\ 2\end{array}\right) \cdot\left(\begin{array}{l}8 \\ 3\end{array}\right)$ generates repeated outcomes). Unfortunately, though, Joshua had not made any connection between the counting processes and the set of outcomes. He did not seem to have a way of grounding the two processes that he was comparing, and thus he mistakenly thought that they were both providing correct answers to the problem.

\subsubsection{3 - Sets of outcomes and formulas/expressions}

In the model, there is one other relationship to discuss. I mention it here briefly for the sake of completeness and to highlight it as a potential avenue for further study. While it could be the case that some counters could utilize/establish the relationship between sets of outcomes and formulas/expressions, I did not explicitly investigate this 
relationship in my study, and I did not find evidence that would help to flesh out the relationship. I conjecture that perhaps for some particularly experienced counters, there may be certain sets of outcomes that could be directly connected to certain formulas or expressions without having to consider a counting process. A possible example of this is

a binomial coefficient $\left(\begin{array}{l}n \\ k\end{array}\right)$. While there is an underlying counting process that it represents (choosing a subset of $k$ objects from a set of $n$ distinct objects), for some advanced counters it can become an expression with encapsulated set-theoretic meaning. Specifically, it can be seen as the set of all possible $k$-element subsets whose elements come from some larger $n$-element set. While my study does not speak to this relationship directly, I maintain that it is a potentially useful aspect of the model. In what follows, this particular direct relationship in the model will not be considered; the sets of outcomes and the formulas and expressions are connected through counting processes.

\subsection{2 - The model and set-oriented thinking}

The relationships between sets of outcomes and counting processes, and the relationship between counting processes and formulas/expressions are both key elements of the model. In my study, I have focused particularly on the relationship between counting processes and sets of outcomes, and particularly how the consideration of sets of outcomes can play into students' counting activity. While I believe that the inherent, natural relationship between counting processes and formulas/expressions is important, in my study it has largely been in the background, and for the most part the students and I 
treated that relationship almost as an assumption. In what follows, I will appeal to this aspect of the model only in cases where this relationship was problematic for the student.

In my study I have primarily looked at students' set-oriented thinking, and my definition of set-oriented thinking can be described in terms of this model. Specifically, I define set-oriented thinking as combinatorial thinking that involves attending to sets of outcomes in some way. While I obviously cannot know for sure what is going on in the students' minds, I can examine their observable external activity (including language, gestures, and inscriptions) and make interpretations about their thinking. I thus take as evidence of set-oriented thinking to be those observable activities and artifacts that suggest students have, in some way, considered sets of outcomes in their work. It can be assumed, then, that when I talk about students' thinking, and more specifically their setoriented thinking, I am referring to my interpretation of what they are thinking based on their observable language and activity.

Most often when I talk about a student using set-oriented thinking, I mean that the student was referring to precisely the set of outcomes that is generated by a particular counting process (or to the set of outcomes whose cardinality is the answer to a particular counting problem). In some cases, though, set-oriented thinking might include the consideration of some other set which is related to the set of outcomes. For example, when a student solves a counting problem by first counting some total set and subtracting of the "bad" outcomes (this strategy is discussed in detail in Section 5.3.1.2), they technically think of other sets in addition to the set of outcomes - namely the total set and the bad set - and they situate their desirable set of outcomes in terms of those other sets. I 
would still consider such activity to be an indication of a student using set-oriented thinking, because the sets of outcomes are closely related to those sets being considered.

As another example of set-oriented thinking that involves not the set of outcomes, but a set related to the set of outcomes, I present Peter's work on the Cards problem. The example below is interesting for a number of reasons, but here I highlight what I take to be his set-oriented thinking. In Peter's work on the Cards problem, he had initially arrived at an incorrect answer of $12 \cdot(12+13)$. He drew the Venn diagram below (Figure 25 ), in which he was trying to describe the number of options he had for face cards that were hearts, and the number of options of face cards that were not hearts. 


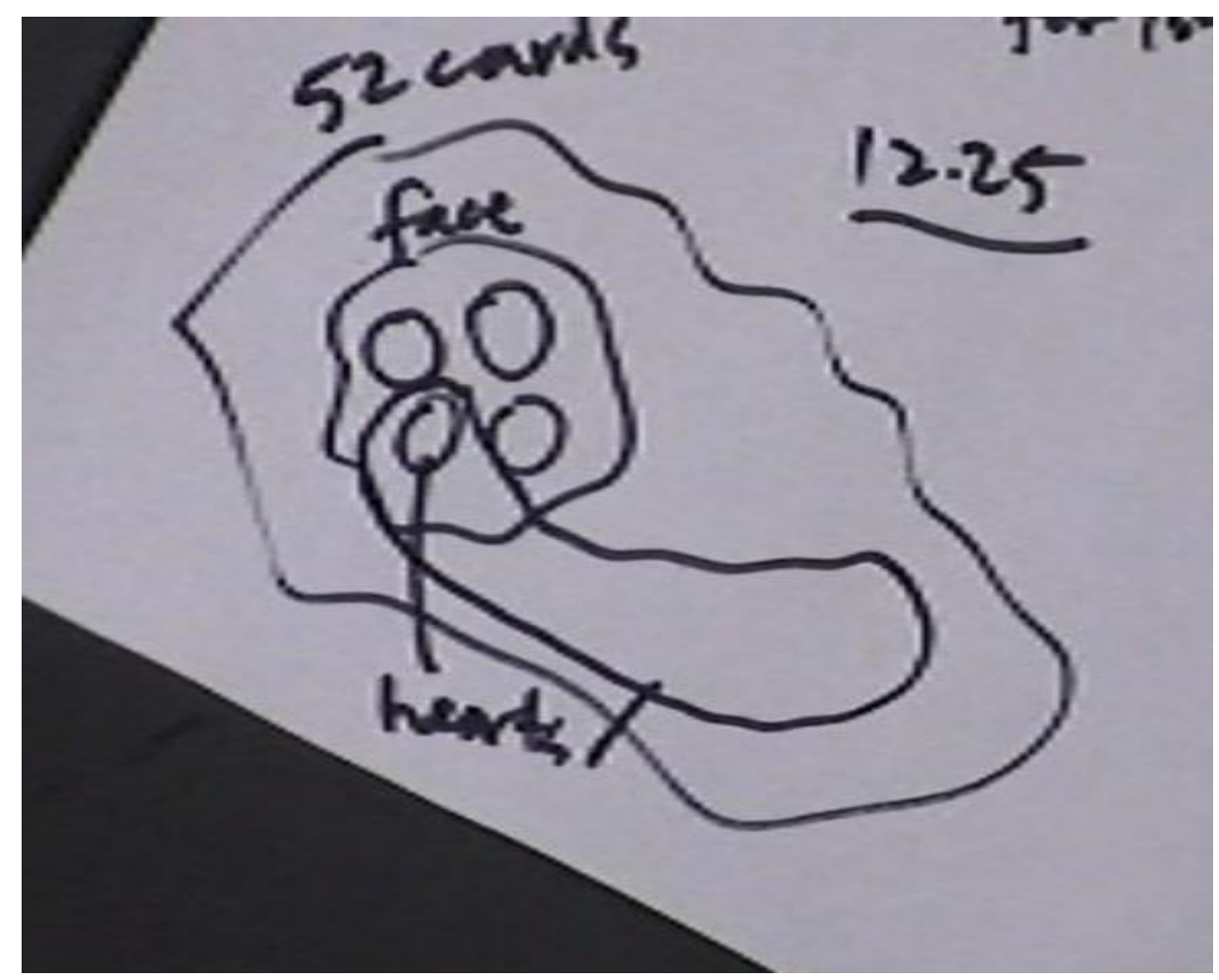

Figure 25

This diagram served to organize the set of options that he had for each card. The set of outcomes that gives the answer to this problem is a collection of pairs of cards, and it can be thought of as a Cartesian product of two sets (the set of cards that are face cards and the set of cards that are hearts). While this Venn diagram does not itself have pairs of cards as its elements, its elements are individual cards that essentially comprise one part of the Cartesian product. I note that his clear, set-theoretic diagram helped him to detect the error in his work and ultimately to arrive at the correct answer. Specifically, his 
drawing allowed him to see that he wanted $3 \cdot 12$ and not $12 \cdot 12$ as the number of pairs of cards that included a heart face and a heart. While this Venn diagram represents a set that is not technically the set of outcomes, I still take Peter's work here to reflect his setoriented thinking - his activity shows that he thought about a set that was a component of the set of outcomes (one of the sets whose Cartesian product would yield the set of outcomes). In sum, then, while most of the examples throughout the chapter will actually refer to some particular set of outcomes, I maintain that my definition of set-oriented thinking includes thinking of these kinds of related sets as well as the set of outcomes to be enumerated.

In the following section I will go on to outline some of the particular ways in which students drew upon set-oriented thinking, but first I provide an example of how a students' set-oriented thinking fits into the model I have described thus far. In the excerpt below, we examine part of Peter's work on the Passwords problem. Peter was in the midst of examining an alternative answer $\left(\begin{array}{l}8 \\ 3\end{array}\right) \cdot 26^{5}$ to determine whether or not it was correct.

P: Okay. Um, so okay this process will sort of, uh, okay so what this process does is it generates something like this (EEEeeeee), right?

E: Mm-hmm.

P: And then I can sort of arrange those E's wherever I want - those big E's wherever I want them to be. Um, it gives me, so, in other words I can, um, I can, so, so all these, oh, um. Ah, that's it. Okay. Yeah. Yeah, certainly if I have all 8 E's it seems to count it twice, right?

E: Okay, how so?

P: Well, okay so let's say, um, you know I pick the first 3 are going to be E's. And then the last 5, let's say just by random chance, happen to be all 5 E's. So I've got 
8 E's in my thing. Well I could get that same result by picking the last 3 to be E's and then having the first 5 just by random chance being E's as well. So, so this is certainly one, so this is a string that's counted twice, yeah. At least twice, actually more, yeah, more than once. So okay, yeah. That at least convinces me that this is an over count.

It is first of all noteworthy that while Peter was initially only given an expression, $\left(\begin{array}{l}8 \\ 3\end{array}\right) \cdot 26^{5}$, he quite naturally (and of his own accord) spoke of it in terms of a counting process that it could represent. This example shows a student who correctly identified a relationship between a formula/expression and a counting process. This example also shows how this relationship seemed to be an assumption for students; Peter did not explicitly discuss how or why a counting process was associated with the expression, but he simply moved on as if it was a natural association. We also see that Peter made a general statement about that counting process generating some outcome. As he drew out a possibility of what the process generated, he wrote out EEE in the first 3 slots and wrote small e's in the remaining 5, yielding the password EEEeeeee. Then he realized that he also could have placed the initial E's in the last spot, and filled the first five slots with E's, so he got eeeeeEEE. Each of these represented to him the all E's password. In the final cell above, he articulated well that the all E's password was going to be counted more than once by the expression $\left(\begin{array}{l}8 \\ 3\end{array}\right) \cdot 26^{5}$, and so he recognized that the expression did in fact over count and was therefore incorrect.

Peter's work here is a powerful example of how the identification of a particular element of the set of outcomes can help someone determine (and justify) why a particular 
solution is incorrect. Peter found that the set of outcomes generated by the process of choosing 3 of 8 slots for E's and then filling any letter into the remaining 5 slots (the expression is $\left(\begin{array}{l}8 \\ 3\end{array}\right) \cdot 26^{5}$ ) actually generated a set with repeated elements. And thus, the set of outcomes that the incorrect process generated did not match exactly (there was not a one-to-one correspondence) with the set of outcomes that were to be counted (the set of all 8-letter passwords with at least 3 E's). Until Peter identified a specific element (the all E's password) that was overcounted, he was unable to explain why the expression was incorrect. In order to come up with particular password that could be useful for him, Peter utilized set-oriented thinking - specifically to think about how a counting process might have generated a password. In this example, Peter made a clear connection between a counting process and an element of the set of outcomes, thus giving evidence of setoriented thinking.

This concludes my introduction to the notion of set-oriented thinking as it relates to the model. My contention is that relationships between sets of outcomes, counting processes, and formulas/expressions are fundamental aspects of counting. Such coordination facilitates a bridging of students' procedural counting processes with their consideration of the set of outcomes to be enumerated.

In this section I described the components of the model and the relationships between these components. In the Conclusions chapter I will further elaborate some of the ways in which set-oriented thinking emerged in the data, and I will frame these findings within the model described above. In the remainder of this chapter I will describe particular 
episodes As I go through these episodes, I will at times relate them back to the model, exemplifying ways in which the model helped me to explain/describe/analyze the data.

\section{2 - Episodes of student work, organized by problem}

In this section, I strive to give the reader a sense of what happened in the data. For each problem, I discuss three students' counting activity in detail. For each of the students, I first provide a brief (one paragraph) overall description of their work on the problem. I then offer a chronological description and discussion of what their work on that problem entailed. In these detailed descriptions, I seek to be fairly comprehensive in my discussions, and I thus include any noteworthy activity by the student, regardless of whether it pertains exactly to set-oriented thinking. I also, however, elaborate upon aspects of student work that are particularly relevant to the research questions and to the conclusions that are drawn in the next chapter. I organize this chronological discussion into sub-sections, labeling each sub-section according to a particular noteworthy incident or theme. At the end of the discussion of each problem, I also identify, among other students who were not discussed in detail, any particularly relevant episodes that occurred, emphasizing major themes or phenomena that relate to my research questions. Finally, I wrap up the discussion of each problem with a brief summary. In the summary I include some general information about each problem, including data about the number of students who solved the problem correctly and the frequency of some common solutions that students presented. 
In this chapter, I primarily seek to describe what happened during the interviews. By giving the reader a sense of what happened, the reader can make judgments about whether they feel my interpretations and analyses (both in this chapter and the Conclusions chapter) are justified. While I want my account of what happened to be straightforward, as I present these results and tell the story there may necessarily be some instances in which I must provide my own interpretations of what happened in the interviews. And, more specifically, as I relate the particular episodes to the model, I will certainly provide interpretation in order to do so. I will make the reader aware of my interpretations as they happen, and will provide some justification for my interpretation. In particular, I may make an interpretation in an overview or a summary of a students' work on the problem, but I will back up that interpretation in the more detailed discussion of that problem. Therefore, the discussion of these episodes will involve my interpretation, but I am making an effort to paint a true picture of what happened in the interviews.

\subsection{1 - The Passwords problem}

The Passwords problem states, "A password consists of 8 upper case letters. How many such 8-letter passwords contain at least 3 E's?" In my discussion of the Passwords problem, there are two major expressions to which I routinely refer. A detailed analysis of the Passwords problem was given in the Methodology chapter, and I discussed two typical responses - a correct case breakdown and a common incorrect answer. For the sake of space and efficiency, in this section I will refer to the correct case breakdown 


$$
\left(\begin{array}{l}
8 \\
3
\end{array}\right) \cdot 25^{5}+\left(\begin{array}{l}
8 \\
4
\end{array}\right) \cdot 25^{4}+\left(\begin{array}{l}
8 \\
5
\end{array}\right) \cdot 25^{3}+\left(\begin{array}{l}
8 \\
6
\end{array}\right) \cdot 25^{2}+\left(\begin{array}{l}
8 \\
7
\end{array}\right) \cdot 25^{1}+\left(\begin{array}{l}
8 \\
8
\end{array}\right) \cdot 25^{0}
$$

as "Expression A" and the common incorrect answer $\left(\begin{array}{l}8 \\ 3\end{array}\right) \cdot 26^{5}$ as "Expression B." When I revisited the problem with students, I typically gave them Expression A first to look at (nobody initially arrived at Expression A), and then once they made sense of that I gave them Expression B.

To contextualize the episodes below, I include some overall data on the Passwords problem. All 22 students attempted the Password problem, and I revisited the problem with 20 of them. Of the initial responses, 5 students got the problem right. This Passwords problem can fairly naturally be broken down into cases, (which could be done in the context of subtracting the bad cases from the total), and indeed, in their initial work on the problem, there were 15 instances in which students utilized or attempted some sort of case breakdown on the problem. In fact, identifying the structure of the case breakdown was not problematic for students - 14 times they correctly identified the case breakdown, but the actual cases were counted correctly only 7 times. It is also noteworthy that all 5 of the students who got the problem right on their first attempt utilized the totalminus-bad approach and arrived at the correct answer

$$
26^{8}-\left[\left(\begin{array}{l}
8 \\
2
\end{array}\right) \cdot 25^{6}+\left(\begin{array}{l}
8 \\
1
\end{array}\right) \cdot 25^{7}+\left(\begin{array}{l}
8 \\
0
\end{array}\right) \cdot 25^{8}\right]
$$

Nobody gave the correct answer 


$$
\left(\begin{array}{l}
8 \\
3
\end{array}\right) \cdot 25^{5}+\left(\begin{array}{l}
8 \\
4
\end{array}\right) \cdot 25^{4}+\left(\begin{array}{l}
8 \\
5
\end{array}\right) \cdot 25^{3}+\left(\begin{array}{l}
8 \\
6
\end{array}\right) \cdot 25^{2}+\left(\begin{array}{l}
8 \\
7
\end{array}\right) \cdot 25^{1}+\left(\begin{array}{l}
8 \\
8
\end{array}\right) \cdot 25^{0}
$$

although some students attempted the structure of adding cases for exactly 3 through 8 E's (but incorrectly counted the cases). Three students gave the incorrect answer $\left(\begin{array}{l}8 \\ 3\end{array}\right) \cdot 26^{5}$, while fourteen students gave some other incorrect answer.

\subsubsection{1 - Peter's work on the Passwords problem}

4.2.1.1.1 - Overall summary. Peter arrived at an initial correct answer by considering all of the possible 8-letter passwords and then subtracting those with 0,1 , or 2 E's in them. Upon revisiting the solution, I gave him the correct case breakdown, which he made sense of ${ }^{29}$, and then I gave him an incorrect answer as well. He explained why the incorrect expression could be a possible answer, and in fact he thought that all three of the expressions (his and the two alternatives) "logically make sense." I then asked how he might more definitely compare the answers; he did a numerical calculation and found that his answer agreed with Expression A, but Expression B was larger than his answer. He then stated that he should look either for something that was overcounted by the bigger answer or undercounted by the smaller one. Peter was able to make articulate statements about what identifying an overcount might entail (finding something that is counted once by the correct answer but more than once by the incorrect one). He attempted to find such

\footnotetext{
${ }^{29}$ I will use the phrase "made sense of" throughout the chapter. If I say that a student made sense of an expression, I mean that they indicated that they could understand the motivation behind such an expression and how it could have come out of some counting process.
} 
a password, but he was unable to do so. I prompted him to consider writing some examples of what he was trying to count; in doing this he introduced notation that enabled him to consider the all E's password. He realized that this password was overcounted by the incorrect solution, and this resolved the numerical discrepancy for him. He thus appealed to one particular password in order to clarify a discrepancy that he could not identify by simply analyzing the processes. In doing this, Peter extended beyond the relationship between counting processes and formulas/expressions and instead emphasized a relationship between counting processes and sets of outcomes. This reflected set-oriented thinking. On this problem there were four other students who, like Peter, initially thought two processes were the same, then appealed to a particular outcome to explain the discrepancy.

\subsubsection{2 - Detailed account.}

4.2.1.1.2.1 - Peter utilizes “total minus bad." Peter began by orienting himself to the problem, confirming that there were 26 letters he had to deal with, any of which could be repeated, and he wrote out 8 dashes. He then stated that he would solve the problem by subtracting some of the passwords from the total (see excerpt below). This statement indicates that he was considering the entire set of outcomes, and it served as a planning step for him as well.

P: Um, so okay, I want to know how many contain at least 3 E's, that's, I'm going to probably, just because the counting is easier, I'm going to probably turn that around, and say I want to know how many contain 2 or 1 or $0 \mathrm{E}$ 's, and then subtract that from the total. 
Peter reasoned about counting these cases as follows, referring to the spots and the number of choices he had for each $\operatorname{spot}^{30}$. He first reiterated his strategy for total-minusbad, and then he went about calculating the total and the cases for 0,1 , or 2 E's in the passwords.

P: The total should be, if there's no restriction on anything at all, the total should be, uh, 26 to the $n$, or to the 8 . Um, because I've got 26 in each slot, so I want to take that minus however many that have, um, so 0,1 or 2 E's.

Um, alright, so if it's got 0 E's, that means that none of the slots can have any, so in that case I have, um, 25 to the 8 possibilities for each of them. If it has one, it can only have one E, then basically I've got to pick a spot where that E will go. And none of the others can have it, right, so there's got to be, so 25, uh, plus 25 to the 7 slots have no E's, and then one of them has an E, and I have to choose 1 out of 8 slots to get it. And then, uh, same for the other, I think. So it's 26 to the $6^{\text {th }}$ will have, so 6 slots will have no E's, um, 25 choices for that. And then I have 2 slots out of 8 where I can put the E's, and there's only one letter that can go in each of those.

At the end of this excerpt, Peter had written out his (correct) answer as

$$
26^{8}-\left(25^{8}+\left(\begin{array}{l}
8 \\
1
\end{array}\right) \cdot 25^{7}+\left(\begin{array}{l}
8 \\
2
\end{array}\right) \cdot 26^{6}\right) .
$$

I asked him to talk me through his work, and again he expressed and explained the notion of total minus bad. Peter's articulation of the "total" suggests to me that he had a sense of the entire set of outcomes. Being able to consider the entire set of outcomes allowed him to structure those outcomes and contributed to him effectively arriving at the correct answer.

\footnotetext{
${ }^{30}$ The "slot" and "choices" language was common among the students. In their original treatment of the problem, there were 34 instances in which students referred to or utilized slots in some way.
} 
P: There's 8 slots, 26 different choices of letter for each slot, so it's 26 times 26 times 26, 8 times, it's $26^{8}$, that's the total. But that counts all the ones that have, you know, any number of E's maybe, um, so I want to subtract all the ones that will have either 0 E's or $1 \mathrm{E}$ or $2 \mathrm{E}$ 's, and so if I take all of them, and I subtract all the ones that have 0,1 or $2 \mathrm{E}$ 's, that should give me the number of all the ones that have 3 or more E's.

I then asked if he was picturing anything (this was a common prompt, discussed in the Methodology chapter), and he wrote out the diagram seen below (Figure 26). As his description suggests, the larger circle represents the total number of passwords. Again this shows how he broke up the total set of outcomes into those that contain three or more E's and those that contain less than 3 E's.

E: Were you, could you describe at all, or even write down, were you picturing anything? Like is there any kind of picture or image that you have in your mind that gets at what you're trying to count, or that would help you with this problem?

P: Kind of, um, it's sort of abstract, um, I was thinking like, you know like maybe a Venn diagram, right. So um, this is kind of like all the passwords, here, that we could have with any letter, and then, I sort of just divided it up and said there's some number of those that would have, you know, like 01 or $2 \mathrm{E}$ 's, and then there's some other number that would have 3 plus E's. And so then I just said well, if I want that, then I can take the whole amount, and I subtract this. 


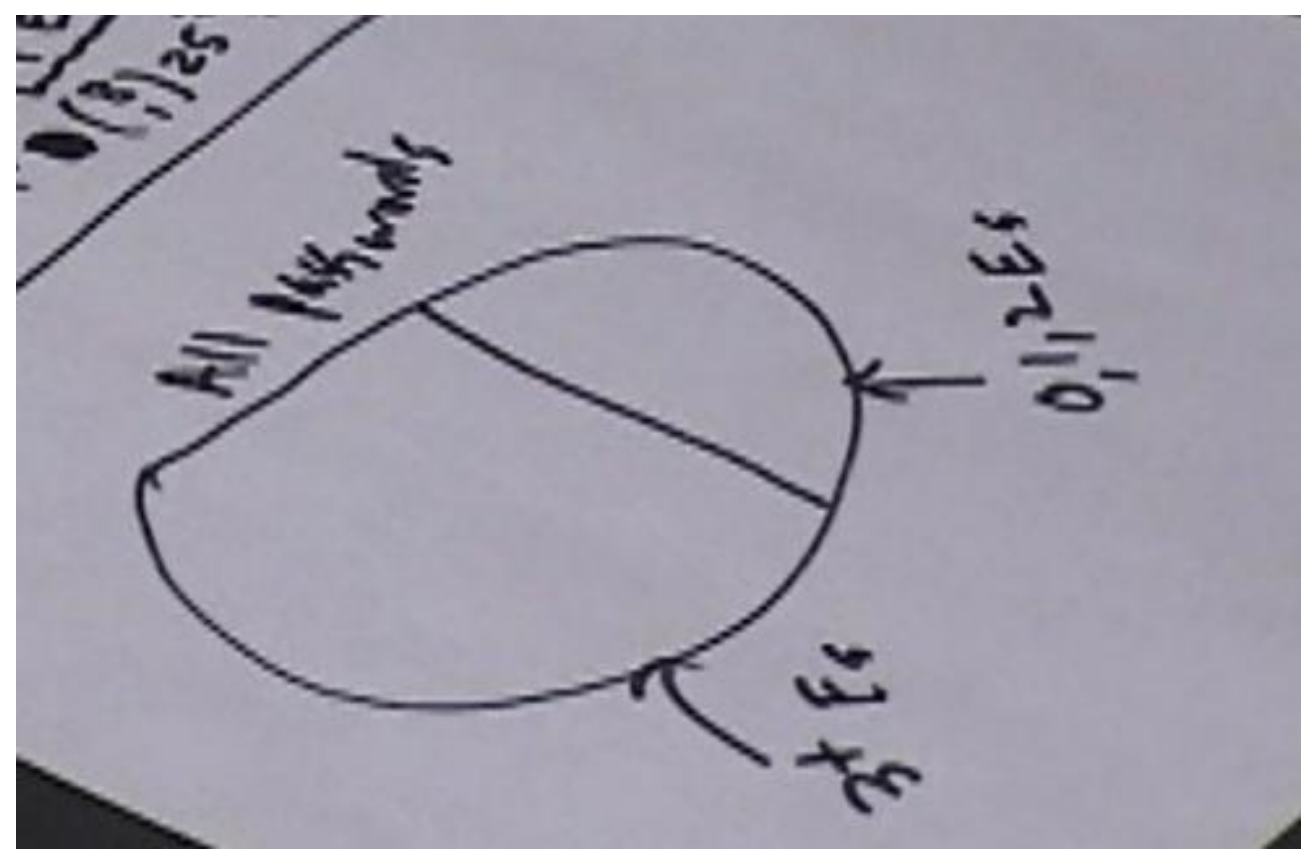

Figure 26

I then asked if he pictured any particular passwords, and he said no. While he had a sense of the length of passwords and of the slots and which letters could go where, he did not initially consider particular examples of what he was trying to count. This concluded Peter's initial work on the problem.

E: Were you picturing at all, any particular passwords, any particular 8-letter passwords?

P: ...No, not at all. I pictured these slots, basically, I thought, okay well I've got 8 slots, and, what can go in those slots, but I never really thought of a specific password at all.

Peter's work to this point suggested to me that he was making a relationship between the counting process and the set of outcomes. In the model, then, his work in this section reflected the relationship below in Figure 27. 


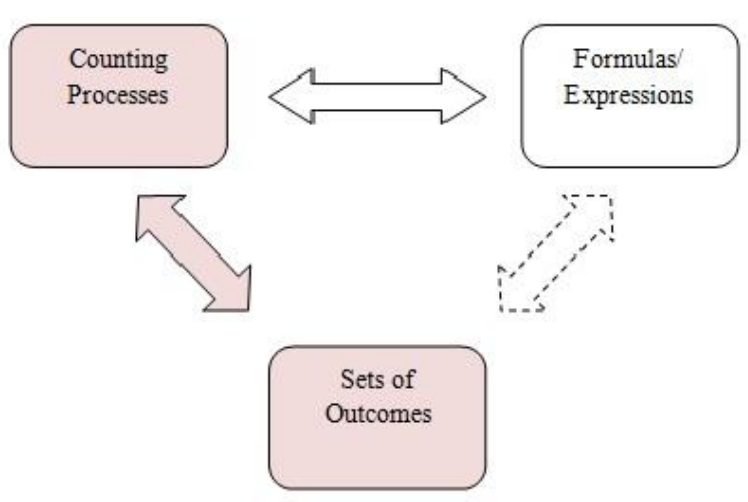

Figure 27

4.2.1.1.2.2 - Peter considers the two alternative answers as the same. We revisited the problem later in the same interview, and I gave him another case breakdown, Expression A. He recognized right away that this was "sort of the way that I said I wanted to avoid, right?" because it involved counting more cases than his approach had, and he noted that the new expression should be equivalent to his original solution. I then gave him an incorrect answer to examine, Expression B. He was not sure initially whether this was correct or not, and he took some time to consider it.

P: Hmm. I'm not convinced that this is a correct solution. I have to think about it... I think what this is saying is, um, choose any letter for - we've got 8 slots, right? So choose any letter we want for 5 of those slots, and then we know we have to have E's for the last 3 slots, so we just pick which 3 out of the 8 to put E's in. I guess this, I guess it's right, I guess it's the same. So it's another way of looking at it.

As he explained what might be going on in the solution, he felt that it made sense and that it suggested another way to look at the problem. Again, he used the slots and choices language as he reasoned about the problem. I consider his work above not to be indicative of set-oriented thinking. He is arguing about the counting process, but he makes no 
appeal to the set of outcomes in any way; instead he analyzes the reasonability of a particular expression by running through the process that the expression represents. This could be reflected in the model as in Figure 28 below, where the red line represents a lack of an appeal to sets of outcomes. We also see that this is an example in which Peter was given an expression, and he was fairly naturally able to determine a counting process that was represented by that expression, as discussed in Section 4.1.1.1 above.

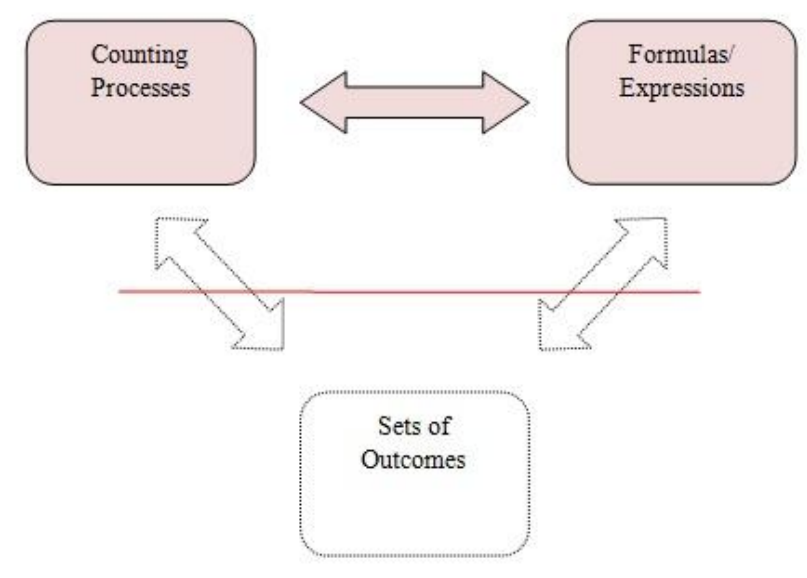

Figure 28

I then asked him to explain how Expression B got at the "at least" constraint, in order to see if he could recognize how the expression handled that part of the problem, and it seemed clear that he understood Expression B as providing a correct answer to the Passwords problem.

E: It satisfies the at least constraint?

P: Exactly. Yeah exactly, because you're guaranteeing that, with this choice here you're guaranteeing that you've got your 3 E's... It's a cleaner way to look at the problem, it wasn't the way I would have thought of it right away though. 
At this point in the interview, I asked him how he might go about further comparing the answers, if he had to be completely certain that they were all the same. We see in the excerpt below that, according to his "analysis" (see the underlined section), he felt that both alternative solutions should have been the same as his original one, and that all three were correct. When he says "analysis," I interpret that to mean his careful examination of both his counting process (which involved total minus bad) and the counting process represented by Expression A (which involved cases), and how each of those counting processes worked. He analyzed the counting process behind each expression, and he felt that they should all be the same. He also suggested that he would compare the values numerically on a calculator, and I asked him to do that.

P: Like for this one for example [Expression B], I didn't think of it. This one [Expression A] I immediately realized is the same type of approach, right? So I'm like okay I understand that approach right away because I already worked through it. And, um, I'm fairly certain that these numbers will add up correctly just because of the analysis. This one [Expression B] I wasn't sure at first, I had to sort of convince myself that all the constraints of the problem would be met by this expression... But then once I did, um, I thought oh yeah that's correct. I mean logically, they all seem solid, I would be fine with all of them... Um, just to convince myself further, like I said just run the numbers in the calculator or something like that.

E: Okay so I might actually ask you to do that, if you don't mind.

He performed the numerical calculation and found that his original answer and Expression A matched numerically as 575,111,451, while Expression B was $665,357,056$. In the exchange below we see that the different numerical answers indicated to him that both could not be correct, and he needed to decide which was 
correct, and why. He first investigated if perhaps the bigger expression was actually overcounting. During this time, it was not immediately clear to him why the answered differed; in fact, it took several minutes for him ultimately to resolve this issue.

P: Um, what is this 575,111,451. Okay, I think I was right, and I think this is right, too. So I think this [Expression B] is an overcount, and, I'm not $100 \%$ convinced that it's an overcount, but, um, the fact that it doesn't add up, and let me do it again just to make sure that it doesn't add up. ... Yeah. So the fact that it doesn't add up to these,

E: And I think that those are the right numbers, just for what it's worth.

P: Oh okay, alright. Well it's interesting, because I'd talked myself into believing this one [Expression B], but now I sort of don't anymore. I have more faith in these.

E: As opposed to, like you said, those undercounting and that being the right answer, right?

P: I think so. I mean, um, there's a nagging kind of doubt in the back of my mind that maybe these are undercounting, but I don't see how...Um, I guess I'd put it sort of like 80-20 (laughs)...maybe these are undercounting, but I really think this [Expression B] is overcounting. So I'm going to try and convince myself that this is overcounting first, um.

In the excerpt above he starts to use language about an "overcount," suggesting to me that he perhaps was starting to be in a situation where he was relating the counting process to the set of outcomes.

4.2.1.1.2.3 - Peter identifies a particular password that is overcounted. I then asked Peter what he meant by an overcount, and in the excerpt below he described what identifying an overcount might entail.

E: And so when you're thinking of an overcount, what are you trying to, what are you trying to do, or what are you trying to find?

P: I'm trying to find a, uh, I'm trying to find a I guess a situation, let's say like an arrangement of letters... where, um, it would only be counted once in an expression like these [his answer and Expression A]... But it would be counted twice or more in an expression like this [Expression B]. 
His language above suggests to me a set-oriented perspective, particularly a coordination of sets and processes. He seemed to indicate that a certain process (which was represented by the given expression) generated some number of outcomes (arrangements of letters, to use his language). He recognized that he was looking for a particular arrangement of letters that was counted only once in one expression but twice or more in another expression. I interpret this as a significant contribution by Peter. He knew that he was trying to identify something counted more than once by the expression that he suspected to be too big, and this exchange suggested to me that he coordinated his counting process with an outcome generated by that process. In terms of the model, his work above provides an excellent example of activity that suggests a student considered the relationship between counting processes and sets of outcomes.

Peter went on to write down 8 slots and to consider if E's were guaranteed to be in 3 of them. He wrote $\_\underline{E}{ }_{-} \underline{E} \underline{E}{ }_{-}$, and then he wrote out XEXXEEXX, where "X can be any letter." He tried to reason about overcounting arising, perhaps, if some of the X's were E's, and then the letters that were originally E's didn't have to be E's, but this was not fruitful for him. After about 3 minutes of work he said, “that doesn't convince me it's an overcount, though...So far I haven't been able to convince myself that this [Expression B] is an overcount." Because of this, he returned to his original answer to see if perhaps he was missing a situation, but he felt that his should be correct.

In an effort to keep things moving in a timely manner, I then prompted him with the following. Since this was a Stage 2 interview, I was interested to see if an activity which 
involved considering particular outcomes might help him proceed in his counting activity.

E: And I guess maybe, yeah, can you give, I don't know, a couple of examples of passwords that that process generates? You know?

P: That this process? [referring to Expression B]

E: Yeah. And I mean that's kind of what you're doing, but I'm just curious, yeah, like, are there a couple of examples you could write out?

I gave this prompt expecting him to write some particular passwords, conjecturing that this might help him in some way. His response was somewhat unexpected; rather than writing out particular passwords with at least 3 E's (such as EEABCDEE, for example), he wrote out what might be considered a prototypical password. He wrote out the first three E's, EEE, and then he stated that he wanted some other symbol to represent other letters that could or could not be E. Since X had previously stood for a non-E letter, he let a lowercase e stand for any letter (which could or could not be an E). So, in response to my prompt, he wrote EEEeeeee, noting that he could arrange the capital E's wherever he wanted. Then, after staring for a bit he then says, "ah that's it," and wrote eeeeeEEE. In the excerpt below we see how he explained his work.

P: And then I can sort of arrange those E's wherever I want - those big E's wherever I want them to be. Um, it gives me, so, in other words I can, um, I can, so, so all these, oh, um. Ah, that's it. Okay. Yeah. Yeah, certainly if I have all 8 E's it seems to count it twice, right?

E: Okay, how so?

P: Well, okay so let's say, um, you know I pick the first 3 are going to be E's, and then the last 5, let's say just by random chance, happen to be all 5 E's... So I've got $8 \mathrm{E}$ 's in my thing. Well I could get that same result by picking the last 3 to be E's and then having the first 5 just by random chance being E's as well. So, so this is certainly one, so this is a string that's counted twice, yeah. At least twice, 
actually more, yeah, more than once. So okay, yeah. That at least convinces me that this [Expression B] is an overcount.

So he identified one particular password (the all E's password) that was counted more than once by the incorrect solution. And, as he put it, this convinced him that the solution given in Expression B was an overcount. Although I do not know for sure (because I did not ask him), I suspect that staring at EEEeeeee likely contributed to him seeing the all E's password. It is possible that this example occurred to him because he used $e$ 's (and not some other letter, like $n$ ) to represent "any letter." As we concluded our work on the problem, I asked how he knew that his solution did not overcount the all E's password, and the following exchange occurred. Although he did not explicitly mention cases in this excerpt, I interpret that there is a connection between his statements below and his case breakdown. By “controlling how many E's there are at each step," he has guaranteed that his cases are, in fact, disjoint.

E: And how do you know that all E password doesn't get overcounted in your example? By your solution?

P: Because, because we're controlling how many E's there are at each step... Like in this one [Expression A], for example, um, this 25 to the 5 is, we're specifically excluding E's from the spots that we haven't chosen to put E's in. So we're controlling the number of E's at each stage.

Peter was finally above to identify what was wrong with the incorrect expression, and he drew upon the set of outcomes to do this. Indeed, it was by drawing upon a particular element in the set of outcomes that he was able to reconcile the error in Expression B. 
This kind of activity is reflected in the model in the relationship between counting processes and sets of outcomes (Figure 27 above).

4.2.1.1.3 - Summary. In Peter's work on this problem, he arrived at a correct answer initially, and he drew upon the set of outcomes to do so. When he was presented with an incorrect alternative, he made some sense of it and suspected that it did the same thing as the correct solution. That is, he could explain why the alternative solution could be a solution to the counting problem, and in fact he thought it was correct. Comparing the processes represented by each expression was not enough to allow him to determine the error (and where the overcounting occurred), and in fact in this comparison he did not draw upon the set of outcomes but looked only to the relationship between the formulas/expressions and the counting processes. Ultimately, though, he returned to considering the set of outcomes and identified a particular element of the set of outcomes (in his case the all E's password) in order to make sense of why the incorrect answer overcounted. There were four other students whose work on the Password problem closely resembled Peter's. Though not all of the particulars of these interviews were the same, the structures were similar; their work was characterized by a) suspecting that the incorrect answer "did the same thing" as the correct one and b) identifying a particular password that was overcounted by the incorrect answer in order to explain the discrepancy.

\subsubsection{2 - Anderson's work on the Passwords problem}


4.2.1.2.1 - Overall description. In this problem, Anderson initially did a case breakdown for passwords containing three through eight E's respectively, yielding $25^{5}+25^{4}+25^{3}+25^{2}+25+1$, suggesting some initial organization of the set of outcomes. In the second interview, I gave him the correct case breakdown, Expression A, and he recognized that the binomial coefficients (which had been absent from his work) allowed for the placement of the E's; he was able to articulate how it provided a correct answer. I then gave him the incorrect answer, Expression B, and he made some sense of it and said that it was a simpler way of approaching the problem; in doing so he drew upon the relationship between formulas/expression and counting processes. He calculated the values numerically and found that they differed, and he went about trying to make sense of the numerical difference. He considered doing the problem "backwards" by subtracting off the bad cases, but he was slowed down by computing those cases. He then went to a smaller case of a 4-letter password that contained at least 3 E's, and he did some detailed investigation of that smaller problem. At that level, he applied both expressions and analyzed them numerically, and he noticed that even with the smaller case there was a numerical discrepancy. It was at this point that he identified a password that was overcounted, and he was able to apply his reasoning to the original problem, thus using the set of outcomes to justify the discrepancy. We concluded with him recapping his work on the problem, and he stated that his work gave him confidence in which answer was correct.

\subsubsection{2 - Detailed Account}


4.2.1.2.2.1 - Anderson arrives at an initial incorrect answer. Anderson began the problem by considering all of the possible passwords. In the excerpt below, we see that he referred to the total and noted that there were $26^{8}$ total 8-letter passwords. He seemed to realize that, in relation to the total, he wanted to find those answers with exactly 3 E's, exactly 4 E's, all the way up to exactly 8 E's. His initial answer was

$$
1^{3} \cdot 25^{5}+1^{4} \cdot 25^{4}+1^{5} \cdot 25^{3}+1^{6} \cdot 25^{2}+1^{7} \cdot 25^{1}+1^{8} \cdot 25^{5}
$$

which he simplified to $25^{5}+25^{4}+25^{3}+25^{2}+25+1$ as he described below. The 1 's in his initial answer represented that he had just one choice for each of the E's, and the 25's represent that he could have any non-E letter in the remaining spots.

A: So if, let's see how many would there be in total? There would be $26^{8}$, which I don't really want to calculate right now, alright, which means I need 3 E's out of 8. So how many passwords would have 3 E's? That means out of however many that is, we would have $1 \cdot 1 \cdot 1$ because it's set to be E's somewhere, and then we would have, um, 25 other letters done 5 times. And then we repeat this, $1 \cdot 1 \cdot 1 \cdot 1$ for if there were 4 , 'cause it says at least, times $25^{4}$, and we continue this, $1 \cdot 1 \cdot 1 \cdot 1 \cdot 1 \cdot 25^{3}, 1 \cdot 1 \cdot 1 \cdot 1 \cdot 1 \cdot 1 \cdot 25^{2}$, then I guess $1^{7}$ times 25 . Um, and then I guess there's $1^{8}$.

So I guess the answer would be $1 \cdot 25^{5}+1 \cdot 25^{4}+1 \cdot 25^{3}+1 \cdot 25^{2}+25+1$. So I guess that's $25^{5}+25^{4}+25^{3}+25^{2}+25+1$. Um, it's a large number.

I asked him what made him add the products together, and his response shows how he thought about the cases; his consideration of such cases contributed to the organization of his broader work on the problem. That is, he recognized that he could think of the problem in terms of six cases, each of which represented counting passwords with exactly some number of E's, and he thus decided to count each case individually and add them 
up to get the total. This discussion concluded Anderson's work on the problem in Interview 1.

E: Yeah, that's fine. Okay, great, and can I ask, so you got those products and then you added them together. What made you add them together?

A: Okay well I'm thinking of 8 different cases. Um, each case would have a different number of capital E's in the password... So one case would be there's one E, one case there'd be 2 E's, one case there'd be 3 E's. So since we need at least 3, I would calculate how many passwords, or how many different combinations would have at least 3 E's - would have exactly 3 E's in them, and that's $25^{5}$, and then I would add together with how many passwords have exactly 4 E's, etc. up until all 8 E's.

The work to this point suggested an implicit consideration of sets of outcomes. He generated a formula from a counting process, and he also seemed to realize that he was organizing the set of outcomes. It seems that he considered both the relationship between counting processes and formulas/expressions and the relationship between counting processes and sets of outcomes in his initial work on the problem.

4.2.1.2.2.2 - Anderson recognizes an error in his original answer. In Interview 2, I first asked Anderson to re-cap what he had done in the first interview. The underlined excerpt below sheds light on how his cases related to the whole, and gives some evidence that he considered partitioning the set of outcomes in some way. Note, as he described his initial solution, he did not change his solution in any way.

A: Alright, um, well the task, um, the problem is that there's a password that has 8 uppercase letters, and how many of, um, how many possible 8-letter passwords would contain at least 3 E's? Um, the way I originally did this problem is I split it up into 8 cases, or 9 cases technically, where the password would contain exactly 0 E's, one E, 2 E, and I excluded those from my, uh, solution.

So I started off with, uh, calculating how many different passwords, or, uh, 
combination of letters would have at least 3 E's, and I did that by, um, basically taking 1 to the 3rd because they're - because 3 of the letters are set, so I don't have any other choice in the matter. And the remaining 5 letters would be any of the other 25 letters in the alphabet. Um, then I continued on with the next case, where, uh, 4 of the letters are E's, so $1^{4}$ multiplied by $25^{4}$ for the 4 remaining letters, and I continue on, um, 5 E's, 3 other letters, 6 E's, 2 other letters, and 7 E's, 1 other letter, and then, all E's.

I then gave him the correct alternative solution, Expression A, and asked him to make sense of it. After Anderson looked at it for some time, we had the following exchange.

A: It does make sense to me, um, I think if I were to compare it to the way I did it, um the way I did it is more of a, if like say the E's were in set places. So I guess I didn't answer the question as I should. Um, for example in my first case where I did $1^{3}$ times $25^{5}$, um, that's assuming for example the first 3 letters are E's, rather than them being in any order.

E: Okay. Okay and so if you had to compare which of the - I mean, so do you think your solution and that solution, are those different numbers?

A: Yes.

E: Okay, and if you had to compare which you think, um, answers the question, like what's the right, you know, right answer, what do you think?

A: I think based on, yeah, this solution, this other solution would be the correct answer, um, because this does take into account that the E's can be in any location in the 8-letters.

In the above episode, Expression A differed from his solution only by the presence of the binomial coefficients before each term. It is noteworthy here that Anderson was able to focus on particular features of the passwords. His language suggests that he considered the format of the passwords and imagined E's being in the first 3 places, and compared that with the situation in which E's were in different positions in the password. Based on his analysis here and his understanding of the new answer, he determined that the alternative expression (Expression A) correctly answered the problem. 
I then gave him the other alternative answer, Expression B. He took some time to consider it, and as he talked through the expression, he justified to himself why it might make sense. The excerpt below shows how he made sense of the alternative answer, and "based on how it works" he felt that it could make sense.

A: Hmm. Okay. Um, this one I think follows along the same pattern where it first accounts for the fact that the E's can be in any location within the 8-letter password, um, where it changes, uh, where it multiplies by $26^{5} \mathrm{I}$ think is trying to simplify the remaining parts of the problem, um, saying that, we don't really care what's in the other 5 letters, they could be E's, they might not be E's, um, it doesn't matter, though, because we've already accounted for 3 of them, which means that any combination, E's or not, of the other 5 letters, would already be taken into account, or would already qualify for this problem. Um, yeah so I guess,

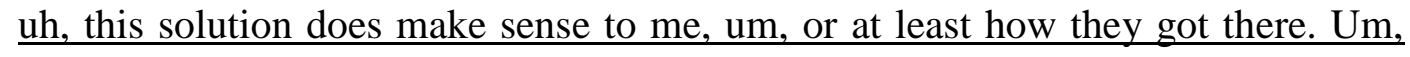
but yeah I think it's sort of a simpler way of approaching the problem, rather than breaking down case by case.

E: Okay. Um, do you think it's an equivalent answer, do you get, um, I mean does it seem like it's doing the same thing that I just showed you?

A: Off the - yeah, off the top of my head, without any calculations, based on how it works, I would say yes...Um, for some reason I have something in the back of my head telling me there might be something off, but I'm not really sure what it would be right now.

In these excerpts, Anderson's language suggested that he was evaluating the process behind the expression, and in his analysis above he seems to be arguing about the process and not considering the set of outcomes (Figure 28 above). He did note some suspicion about it, but he could not articulate what specifically might have been wrong with the answer. Thus, we see that based on a description of how the problem worked, Expression B seemed to Anderson like a reasonable possibility. 
I then proceeded to ask him which answer he preferred, if he had to choose one that he felt most comfortable with, and without any further analysis, he noted that he felt more comfortable with Expression A because the case breakdown felt more precise to him. He then found the exact answers on a calculator, and he found that Expression B was much bigger. He commented that neither answer took into account the cases that it should not have - namely those with two, one, or no E's. This suggested some thought about the entire set of passwords, and making a distinction between those that have at least 3 E's (and that satisfy the constraints of the problem) and those that have fewer than 3 E's.

I encouraged him to continue to think about why the discrepancy occurred, and he continued to work on that aspect of the problem. For a time he considered what might happen if he tried "to work at the problem backwards," where he would subtract from the total the cases where he has zero, one, or two E's. This excerpt provided evidence that he was conceiving of the total number of passwords and subtracting those he did not want (thus utilizing a total minus bad strategy). He tried this because he wanted some other way to think about the problem, and the total minus bad strategy gave him an additional way to approach and think about the problem.

A: What I'm trying to do right now is I'm trying to work at the problem backwards. Um, where I take the total number of possible 8-letter combinations, and then I subtract the cases where the password doesn't meet our criteria of having at least 3 E's. Um, so in terms of total passwords, there's, uh, $26^{8}$ total combinations, 'cause there's 26 letters, and there's 8 , uh, it's 8 characters, or 8 letters. And then the case where one of them would be an E, or the case where none of them would be an E is, um, $25^{8}$ characters because it's the same - it's any letters for all 8 , and there's 25 letters total. Then the next case where there's only one E, the E can only be in one of 8 locations, multiplied by uh, the remaining 7 positions filled by any 
of the 25 letters, so that's $25^{7}$, subtracted by another - in the case where there's 2 E's, which for some reason is not coming to mind, um, I don't know if I'm really going to brute force this, I guess. Brute force is so inefficient.

His language above suggests that he had a sense of what he wanted to do - to subtract off those bad cases. However, in the underlined section above, we see that he had a difficult time coming up with the case that has exactly 2 E's in the password. He considered it for a while, but he ultimately abandoned the idea of total minus bad because he could not properly compute that one case; he did not use the existing correct solution in front of him - Expression A - to find the "exactly two" case.

Next, I brought him back to comparing the two expressions, and I asked him which answer he suspected to be correct, if he had to guess. As we see below, he thought Expression B was incorrect. His reasoning this seemed to have had more to do with the fact that Expression A was a case breakdown and Expression B was not; it is as though he inherently trusted the case breakdown more than the slicker, shorter solution. He suspected that by skipping steps, "something's not being accounted for."

E: So do you have a hunch - or, which you think is right? Like, you know, do you think it's the case, if one of those two is right, uh, which one do you think is off?

A: Um, my gut feeling tells me that the second one is off, but for some reason I'm not - yeah I'm not coming to an answer as to why.

E: Okay and I guess I'm curious, like, what, um, and maybe this is what you're trying to but, yeah, how could you, I don't know, convince yourself that that's the one that's off. If you had to, yeah, try to decide which of those is right?

A: I feel like, it's because the first one handles things on a case by case basis, which is what I tend to do. The second one sort of pulls it all into one case and tries to simplify it, but I feel like it might create like some room for error somewhere, like something's not being accounted for. Um, I guess I would call it some bug in the system. 
This issue of judging whether or not 'slick' solutions were better came up with other students, too. The opinions varied, however; some, like Anderson, trusted the longer case breakdowns, while other students seemed to prefer more succinct answers.

4.2.1.2.2.3 - Anderson carefully examines a smaller case. As Anderson continued to work, he decided to truncate the situation to examining the number of 4-letter passwords that contain at least 3 E's, rather than 8-letter passwords that contain at least 3 E's. In particular, he had written down some slots and had put 1's where an E would go, and 25's where a non-E would go. After writing a couple of these slots, he decided to look instead at only 4 slots instead of dealing with all 8 . He noted that there were 25 options for any placement of 3 E's, and he explained again that there were $\left(\begin{array}{l}4 \\ 3\end{array}\right)$ ways to place 3 E's in the 4 slots, and then 25 choices for the remaining letter. And then he considered the case in which all 4 letters were E's, and he stated that there was just one way to do that (this gave him an initial answer of 101). In this activity, I interpret that Anderson was relating counting processes to expressions in the context of a smaller case.

Then, Anderson engaged in some numerical computation for several minutes. He went from the 4-letter password scenario to looking at 5-letter passwords. Though he played around with these passwords for a while, it was not clear that this was fruitful for him. In the midst of this computation, I made the following intervention, asking him which expression (A or B) he was using as he was performing the calculations (I did this 
to keep us on track; we had already spent a good deal of time on the problem, and I felt like the 5-letter case was getting away from him).

E: What you're finding there, like for your four case and your five case, which of the two solutions was that implementing?

A: Uh, the first one [referring to Expression A].

E: The first one, okay.

This intervention ended up being significant; in response to my question, he abandoned his work on the case of 5 letters, and instead he tried the 4 letter problem with the second alternative answer (Expression B). When he did this, he arrived at $\left(\begin{array}{l}4 \\ 3\end{array}\right) \cdot 26$ which gave an initial answer of 104. Expression A had given him an answer of 101, and thus he noted, "the difference is already there."

A: Uh, if I was going to translate to the second one then it becomes, uh, in the 4 case it becomes c $(4,3)$ equals 4 , but since the last one doesn't matter, it's 26 , which is, uh, blah, 104. Well, let's see. Uh, what was the total on this? It was 100 , and 1. The difference is already there. It's 101, versus 104 . Um, okay well since there's already a difference here in the half case, where I'm only taking 4 letters instead of 8 ...

This numerical difference of 101 and 104 was small enough for him to be able to consider in detail, and he proceeded to examine the difference here more closely. The following episode was key for him. First, he said "I have E, E, E, A through Z. Which is equal to 26." And he wrote down E E E A-Z. Then, he said, “Then I have another E, E, A through Z, E, which is another 26," and he wrote E E A-Z E. Then he said, "And since I do this 4 times, I have 4 times 26, which is 104, okay, which would suggest that the second one (Expression B) is correct." Then, nearby, he wrote out E E E A-Z-E, and he 
said, "I have 3 E's, then I set them to any 25 letters, so let's see, A through $Z$ minus E. And so I have 100 different ways to do that." Then he wrote E E E E and noted "But then I also have 4 E's, and there's only one way to do that." His reflection on this discovery is seen below. He identified a particular password (the all E's password) that was counted too many times by the incorrect solution. The underlined portion suggests a coordination of processes and outcomes - that he understood that the incorrect process was counting some passwords too many times. This is again highlighted within the model as the relationship between counting processes and sets of outcomes.

A: Oh, there we go, that's where the difference is. So the difference is, um, yes there's 26 different ways to arrange it so that the first 3 letters are E's, and then the last one can be any of the 26 letters. And then there's another way to arrange it so that the first 2 and the last letter are E's, and the 3rd letter is any letter between A and Z, except if the third letter is an E, it's exactly, it's the exact same case as if the $\mathrm{E}$ was the last letter in the first case, which means it's counting multiple passwords twice.

I asked him to explain again what had clicked for him, and he described the general phenomenon that happened.

A: Okay, I wrote this out, and then I thought well, okay that makes sense. And then I wrote this out and I was like, but that also makes sense! So what's going on? And then I realized well, I'm, okay so I'm not going to count the E here, but there's only one way to arrange 4 E's here. And then I looked at it and thought, but, wait, if this is an E, then all 4 are E's, so that's fine. But if this is an E, then all four of these are E's, but that's exactly the same as what we just did, which means it has to be $26-1 . .$. So the first one's right, but the second one is wrong only because it counts the same passwords multiple times.

His first underlined statement above is noteworthy - he acknowledged that as he wrote both answers out, they both made sense to him. It was when he was able to relate each 
process to what they were generating (the second bit of underlined text) that he ultimately realized that the one process was counting the all E's password more than once.

After this, I asked Anderson a couple of questions as a follow up. In the underlined portion below, he gave some sense as to why it took him a while to explain what was wrong with the problem. It seems that just writing down the range (either $\mathrm{A}-\mathrm{Z}$ or $\mathrm{A}-\mathrm{Z}$ excluding E) was not enough for him to see what was happening. His brain was "too lazy to come up with a specific example," and it was not until he came up with that particular example that he could identify the error. This is an insightful self-reflective statement. Indeed, he was able to identify that a key step in seeing the issue was writing down a specific example instead of just writing down a range of choices.

A: And I was like, oh, the problem - the two methods still come up with different answers, so something must be off on some fundamental level somewhere. Uh, so I realized, well, since my brain's not all that math oriented, I guess I'll just like write it out and see where I go, uh, so let's come up with a few examples, so I was like EEE, and I was like, well, my brain's too lazy to come up with a specific example, so I guess I'll just write down the range, and then I should be okay.... Um, and I guess it's that step that my brain kept skipping due to laziness, (chuckles) that made me overlook that one problem.

As one final example of his emphasis on the relationship between counting processes and sets of outcomes, I had asked Anderson to explain, one more time, what he meant by something being overcounted in the original 8-letter Password problem. Below, he expressed a particular example (the all E's password), explaining how that password was counted more than once by Expression B. 
A: So if we go through the problem once, we have, we set the first 3 letters to be E's. Okay? And then we'll set the remaining ones to be E's. And that's fine, it's, you know, it's a valid password, it fits our criteria just fine [writes E E E E E E E E].

E: Mm-hmm.

A: So that's one. Um, now another combination of $\left(\begin{array}{l}8 \\ 3\end{array}\right)$ would be, let's say, E E something and then E [writes E E _ E]. Well hey let's fill the remaining 5 letters with all E's again, [does this, yielding E E E E E E E E].

Sure, that's another valid password, I mean, it meets the criteria, sure why not? The problem is since they're all upper case letters, um, and like I said the lower case is just for explanation, these two passwords are considered different, because this solution only focuses on where we're placing the $3 \mathrm{E}$ 's, um, and how many different ways we can arrange that. The remaining 5 letters can be anything we want, but if they happen to be E's, then we may end up counting the same password twice as we do here. So this would actually count as two separate passwords [according to Expression B], when in reality they're just the same password.

4.2.1.2.3 - Summary. On a number of occasions Anderson showed evidence of being able to coordinate a counting process with some set of outcomes. In particular, he identified a specific element (the all E's password) that was counted more than once, and he engaged in very involved work with a smaller case in order to identify such an element. In his work, he also displayed an unusually high amount of self-reflection; he was remarkably engaged and was up to trying a variety of strategies. Even if tasks were monotonous, Anderson showed a great deal of patience as he worked through a smaller case in careful detail. Anderson's work shows the value of being able to coordinate the relationship between counting processes and sets of outcomes, and he displayed an ability to do so in meaningful and productive ways. Indeed, he was effectively stuck in his work on the problem until he connected the counting process to an actual outcome; after that point he 
was able to reason more coherently about the problem. Anderson's work on this problem is representative of a handful of students who similarly examined small cases. There were 19 instances in which students investigated smaller cases in some way.

\subsubsection{3 - Kristin's work on the Passwords problem}

4.2.1.3.1 - Overall description. Kristin initially arrived at an incorrect answer; she broke the problem into cases, but she counted each case incorrectly based on an assumption that repetition was not allowed. She stated that she had not considered any particular examples of what she was trying to count, and my interpretation is that her work did not indicate that she coordinated a counting process with a particular set of outcomes. When we revisited the problem, I first gave her the correct Expression A, and she was able to explain it and determine why it was right and her initial answer was wrong. I then gave her the incorrect Expression B, and while she could explain the process that the expression represented, she suspected that it either over or under-counted. She spent some time deliberating the discrepancy, but she was ultimately unable to determine why the incorrect answer was wrong. Throughout her work, while there were some instances that suggested implicit set-oriented thinking, such thinking was not prominent, and she appealed primarily to her own intuition or to a counting rule she had learned. Indeed, I

would characterize her work as being almost completely restricted to the relationship between counting processes and formulas/expressions, and not drawing upon sets of outcomes.

\subsubsection{2 - Detailed account}


4.2.1.3.2.1 - Kristin arrives at an incorrect first answer. Kristin began by writing out 8 slots, and she stated that she could have 3 through 8 E's, so she "would do it by cases." She proceeded to talk through her solution, but there did not seem to be clear evidence that she considered the set of outcomes. Her initial answer was

$$
\left(\begin{array}{l}
3 \\
3
\end{array}\right)\left(\begin{array}{l}
25 \\
5
\end{array}\right)+\left(\begin{array}{l}
4 \\
4
\end{array}\right)\left(\begin{array}{l}
25 \\
4
\end{array}\right)+\left(\begin{array}{l}
5 \\
5
\end{array}\right)\left(\begin{array}{l}
25 \\
3
\end{array}\right)+\left(\begin{array}{l}
25 \\
2
\end{array}\right)+\left(\begin{array}{l}
25 \\
1
\end{array}\right)+\left(\begin{array}{l}
25 \\
0
\end{array}\right)
$$

she explains her work below.

K: And then, so if I have 3 E's, I would do that first. And I have, uh, um, 3 choose 3, I think, and then I have to pick 5 more so take the E out of there, so 25 choose 5. And then the second case would be if I pick 4 E's, so 4 choose 4, and then 25 choose 4 , and I add them because, uh, they're cases. So they're not connected to each other, is how I think about it... So then there can be 5 E's, so I do 25 choose 3 , and then there can be 6 E's, so that would be 25 choose 2, and 7 E's, 25 choose 1. And then 8 E's would just be 25 choose 0 . Is that right?

Right after she arrived at her initial answer, she made the following statement about total minus bad.

K: Or I would think about it as doing, since it's at least 3, I would do 1 minus, um, having 1 or 2 or none, which would probably have been easier... But I always do that second after I do cases.

The above exchange is noteworthy because it shows that she conceived of an alternative approach to the problem (subtracting some of the passwords from the total number of passwords). Her language also suggests that total-minus-bad was a strategy that she often utilized, particularly after she had already considered a direct case breakdown. It is hard for me to tell, however, whether this instance of total-minus-bad actually points to a consideration of a set of outcomes, or whether it suggests a strategy she had learned and 
employed independently of a consideration of the set of outcomes (particularly given the underlined statement above). She did not expound any further on this strategy at the time.

After she worked on the problem initially and arrived at her answer, I asked her some follow-up questions. I first asked her to explain more clearly why she chose to use combinations for the problem, and her response below shows that the binomial coefficients represented choosing non-E letters to be in the password. (We will see later that she had not considered that repetition was allowed, which explains her decision to use combinations).

E: Okay so, let's look at maybe the - that 4 choose 4 times 25 choose 4, so what are, what is that doing to you, what are each of those combinations getting you?

K: So, 4 choose 4 gives me my 4 E's, and the 25 choose, I have 4 E's and 8 total letters, so I have 4 letters left to pick. And only, um, I want exactly 4 that time, so I only have 25 letters left to pick from, So I pick 4 from those 25 I have left.

$\mathrm{E}$ : Okay and so the 25 is excluding what?

$\mathrm{K}$ : E.

E: The E, okay. Okay.

K: 'Cause I want either exactly $3,4,5,6$, 7, or 8 .

I then asked her to explain why she did cases. In her response below, she indicated that she "always" prefers to use a case breakdown, but she did not justify mathematically why she broke the problem into cases. She drew upon her own experience with counting problems and "at least" problems in order to come up with the cases strategy.

E: Okay. Sure. Um, and can I ask what made you, what made you think to do cases?

$\mathrm{K}$ : 'Cause it says at least 3, so I know I can have up to 8, so I always do cases normally when I see that I can have multiple ways of doing it.

I asked her to explain some of the specifics of what the expressions represented. She somewhat hesitantly stated that she chose combinations because she was "pretty sure 
order doesn't matter with combination," but she did not provide a clear reason for how she determined whether order matters. As she put it, it was more based on her "gut."

E: Okay. Cool and then could you explain maybe one or two of those - the actual terms that you're adding up. So what is that counting for you?

$\mathrm{K}$ : So I'm doing the combination ones, because, um, I'm pretty sure order doesn't matter with combination.

E: Mm-hmm, okay. And why, why do you want order - you don't want order to matter or you do want order to matter?

K: Uh, no, well. No, I don't want order to matter.

E: Okay, and how come?

K: I'm not sure about that one (laughs)... That I just kind of go off my gut for it, on the ones that don't specifically say order matters or it doesn't matter.

I then asked her if she, at any point, pictured any particular passwords.

E: Okay. Cool, and were you at any point, uh, picturing any particular passwords, or did you, you know,

K: No.

E: Were you thinking about any kind of particular thing? Okay.

$\mathrm{K}$ : I wasn't.

E: You wrote the slots down, is that kind of just to, is that how you typically start that kind of problem, or is that just to give you a sense of...

K: Yeah I always do, when I have, um, like an option of 8 or 7 or whatever, I always put the slots down, kind of so I can imagine how many I need, and see that I can either have 3 or 4 E's or 5 E's, 6 E's, or all E's.

Kristin noted that she did not consider particular passwords. She noted that the slots helped her to imagine how many letters she needs (although she did not specifically say that she means letters), and that drawing out slots is a strategy that she frequently uses.

In this section, we see that even her use of cases and total-minus-bad do not clearly reflect a consideration of the set of outcomes. Her reliance on her intuition suggests that 
counting for her entails counting processes and formulas/expressions, but does not necessarily need to involve the outcomes in a given problem.

\subsection{2 - Kristin examines an alternative answer and detects an error in her work.}

When we revisited the problem, we looked again at her original answer,

$$
\left(\begin{array}{l}
3 \\
3
\end{array}\right)\left(\begin{array}{l}
25 \\
5
\end{array}\right)+\left(\begin{array}{l}
4 \\
4
\end{array}\right)\left(\begin{array}{l}
25 \\
4
\end{array}\right)+\left(\begin{array}{l}
5 \\
5
\end{array}\right)\left(\begin{array}{l}
25 \\
3
\end{array}\right)+\left(\begin{array}{l}
25 \\
2
\end{array}\right)+\left(\begin{array}{l}
25 \\
1
\end{array}\right)+\left(\begin{array}{l}
25 \\
0
\end{array}\right)
$$

and I asked to describe again what she had done. As she looked back at her work, she was unable to make sense of the binomial coefficients like $\left(\begin{array}{l}3 \\ 3\end{array}\right)$ or $\left(\begin{array}{l}4 \\ 4\end{array}\right)$ that she had written in front of some of the terms. She had started to change her answer to

$$
\left(\begin{array}{l}
25 \\
5
\end{array}\right)+\left(\begin{array}{l}
25 \\
4
\end{array}\right)+\left(\begin{array}{l}
25 \\
3
\end{array}\right)+\left(\begin{array}{l}
25 \\
2
\end{array}\right)+\left(\begin{array}{l}
25 \\
1
\end{array}\right)+\left(\begin{array}{l}
25 \\
0
\end{array}\right)
$$

but then I asked her if she was assuming whether repetition would be allowed for the remaining letters. With this question, I had not intended to push her toward a particular train of thought, but because of how she had described her use of the combination formula, I was curious about how she was thinking about that aspect of the problem and suspected that she was thinking that repetition was not allowed. The excerpt below shows our discussion of repetition, including how she changed her answer.

E: And are you assuming that there's repetition allowed for those other letters?

$\mathrm{K}$ : Yes, I think.

E: So like I could have EEAAAA and that would be okay.

$\mathrm{K}$ : Then I wouldn't do it like that. Are they allowed, repetition?

E: Um, yes.

K: So, again,

E: Was that assuming that they, that it wasn't allowed? 
K: Well I don't know, because I get confused with using combinations a lot of times with like the letters... So I think I would have just wrote $25^{5} \ldots$ So I can have, assuming it's not an E, because I've picked my 3 E's, and technically I could do times $1^{3}$, because that's my E's. And then I'm adding, so $1^{4}$ if I have 4 E's, $25^{4}$, and then $1^{5}$ if I have 5 E's, $25^{3}, 1^{6}, 25^{2}$, and then $1^{7}, 25$, times 25 , and then plus $1^{8}$. I like that better...I wasn't taking into consideration that you could have AAA.

In this discussion I likely gave her too much information (particularly when I said "So like I could have EEAAAA and that would be okay"), but I was anxious to proceed in the interview. It is noteworthy that she asked if repetition was allowed; other students determined whether repetition mattered by considering the objects they were counting (passwords). While I cannot draw firm conclusions from this, my interpretation is that it seems as though her decisions about whether order matters or does not matter were not based on considering the objects she was counting (passwords), but rather on a clue in the wording of the problem or on some prior experience she had with counting. After this, she raised the idea of total minus bad again.

K: That's what I would do. But then I was thinking also that I might be able to do, I've never, I'm not confident with these, but if I were to do just 26 choose, oh, so $26^{8}$, which is the total ways that I can arrange any 8 letters... And then I would want to subtract something, so I was thinking if I subtracted the no $\mathrm{E}$, or the no E's, the $1 \mathrm{E}$ 's, if there's no E's, if there is $1 \mathrm{E}$, and if there are $2 \mathrm{E}$ 's, then that would give me the same thing.

E: Mm-hmm.

$\mathrm{K}$ : So I would have done, so $26^{8}$ minus, so no E's would be $25^{8}$, plus $1 \mathrm{E}$ is $25^{7}$, plus 2 E's $25^{6}$. I would assume those would give me the same thing.

In the excerpt above, we see that Kristin considered her original case breakdown to be equivalent to a situation in which she subtracted the passwords with 0,1 , or 2 E's from 
the total number of passwords. This excerpt is an example of something that I took to be a reference to (but not an implementation of) a total-minus-bad strategy, because it indicated that she could think of breaking up the total number of passwords in an alternative way (and organizing them in a potentially useful manner).

I then asked her to make sense of the alternative Expression A. She recognized the case breakdown, and she realized that the binomial coefficient $\left(\begin{array}{l}8 \\ 3\end{array}\right)$ represented choosing 3 of 8 spots in which to place E's. The excerpt below shows her ability to examine an expression and to determine a counting process that could underlie that expression.

K: Well that makes sense to me, too. So,

E: Okay what are they doing here?

K: So they did, um, they did it case-wise, too, so you can have 3 E's, 4 E's, 5 E's, 6 E's, 7 E's, or 8 E's. And then they're picking their E's in a spot first, so 8 choose 3 , because you have 8 spots, putting 3 E's anywhere, and then in the remaining 5 spots $26^{5}, 26$ options for each.

As we continued to discuss her work, she never considered the possibility that the two expressions might represent equivalent numerical values (Expression A is clearly bigger than her answer, because it had additional binomial coefficients in each term, each of which was bigger than one), but she seemed to think that the two expressions both made sense, and that she felt like "they're both right."

E: Okay, and what do you think about that? Or what do you think is...how does that compare to your answer?

K: Well it'd be bigger, and I want to say that that's right. But I also felt like mine was right, so then when I see an answer that actually does make sense to me, and my answer makes sense to me, I feel like they're both right, but they're not the same (laughs). 
E: Okay so what do you now to try to decide which one's right, and to make sense of that?

$\mathrm{K}$ : Mmm. Well, in mine, what is 8 choose 3? So that gives me 8 factorial over 5 factorial 3 factorial, which is $8 \cdot 7 \cdot 6$, over 3 factorial. Gosh it just makes me second guess my answer, because it seems completely logical.

I proceeded to ask her more questions as she made sense of the relationship between her answer and Expression A. The exchange below is noteworthy. It represents some coordination of sets and processes, but the coordination is not fully formed and articulated. I asked her what the $\left(\begin{array}{l}8 \\ 3\end{array}\right)$ was doing in Expression A, and in answering, she offered a quasi-example; Figure 29 shows what she referred to in the excerpt below.

E: Okay well what's the 8 choose 3 doing?

K: So it's,

E: Because that's the only difference, right? You have a 1 and they have an 8 choose 3.

K: So they're having more ways to place their E's than I do. So they're taking into consideration, I mean I was just saying, you know, 26, 26, 26, 26, 26, and then 1 $11 \ldots$ And I was saying that that's the same as, as, like, $111,26,26,26,26$, so I think in my answer, actually I'm almost certain in my answer these two are the same. And in theirs, they're not... And since it's a password, these shouldn't be the same. So I would think, I would go with his or hers, whosever it is, because I wasn't thinking about it that the E's mattered where they went. 


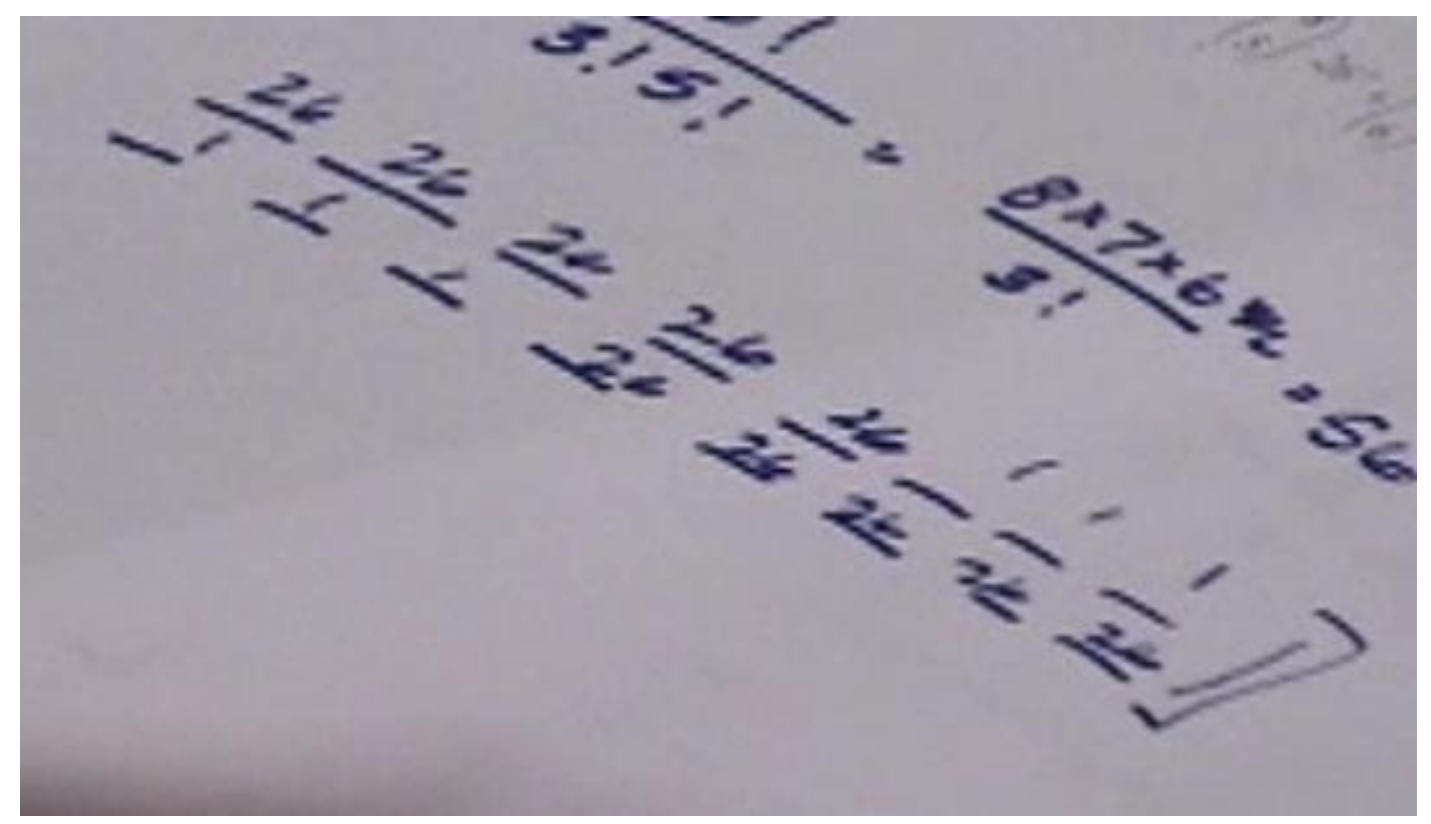

Figure 29

In Figure 29, we see that Kristin said she had considered 2626262626111 as being the same as 1112626262626 , but that the $\left(\begin{array}{l}8 \\ 3\end{array}\right)$ in Expression A made those two situations different. This is insightful, because indeed the placement of the 1 's in $\left(\begin{array}{l}8 \\ 3\end{array}\right)$ ways is important. It is not exactly an example of an outcome, however; the 1's and the 26's represented choices she had for each particular position in the password, but not a particular password. In some senses, comparing two situations in which the choices are jumbled up does not make a lot of sense. I assume that she meant that she had considered that two different arrangements of the E's were the same in her answer but different in Expression A, but this is a step away from comparing something like AAAAAEEE and 
EEEAAAAA $^{31}$. Regardless of whether she considered jumbling the choices or considered a particular example, though, this consideration proved to be useful for her. As she considered the different arrangements of E's in her password, she realized that she actually wanted to count passwords in which the E's were arranged differently as distinct from each other. And, ultimately this allowed her to make sense of the alternative Expression A and to decide that it, and not her original answer, was the correct solution. Even though she considered 26's and 1's (as opposed to, say, thinking of a particular element of the set of outcomes), she was referring to some feature of the passwords (this marks a change from the discussion above, when she did not refer to particular aspects of the password in order to determine whether repetition mattered).

As she continued to articulate the difference between her original answer and Expression A, she restated her thinking. There are three noteworthy points in the following exchange, which I will discuss below.

$\mathrm{K}$ : $\quad$ So I just was trying to figure it out, just thinking if I drew 2 different examples I might be able to see something... So, because I did just 1 times 3 , times $25^{5}$, so in my case these would be the same. 'Cause this is still $25^{5}$ and $1 \ldots$ to the $3^{\text {rd }} \ldots$ and in this case they're doing, well before that, then I looked at these 2 and thought back to, well I have a, it's a password... Meaning ABA is not the same as BAA, because you're not going to get in. So these aren't the same. They can't be the same, and I'm counting them as the same.

First, we see some evidence of a relationship between counting processes and sets of outcomes - that her particular answer represented a process that counted two outcomes as

\footnotetext{
${ }^{31}$ We note that there is one further minor error in the expression. Specifically, the 26 's should be 25 's. The student later noticed this and ultimately changed the 26 's to 25 's.
} 
the same that actually "can't be the same." She had related her process with generating some outcomes that were actually different, but that had been considered the same by her process. Second, it is interesting that she referred to drawing "two different examples." This was in reference to her work in the above excerpt and picture, and it meant that she considered her 2626262626111 and 1112626262626 to be examples; they served as an example for her in her preceding work, even though these were not particular instances of what she was trying to count. (I consider them to be more like prototypes of examples, and yet they served the purpose for Kristin of shedding light on why her initial solution was incorrect). Third, we see another instance of how a particular example comes into play, she says, "It's a password, meaning ABA is not the same BAA, because you're not going to get in." Here she actually uses the properties of passwords in general to make an argument about whether some passwords are actually identical or not. There is thus a sense that she could make sense of the $\left(\begin{array}{l}8 \\ 3\end{array}\right)$, and she could argue about why the new solution was correct. At this point in the interview, she had compared Expression A to her original solution, had made sense of Expression A and had decided that she thought that was the correct answer (and why exactly it differed from hers).

4.2.1.3.2.3 - Kristin struggles to reconcile differences between Expressions $A$ and $B$. I then gave her Expression B; while she did not like it initially, it was not immediately clear to her what made it wrong, and even whether it was over or under counting.

$\mathrm{K}$ : Mmm, so, 8 choose 3... Okay so, I think they would be over-counting. Or undercounting. They're over or under-counting, one of them. 
She took some time to consider whether Expression B was over or under counting, and in doing so she wrote down some passwords. First, she wrote $\mathrm{E}_{-} \mathrm{E}_{-} \mathrm{E}_{-}{ }_{-}$. Below it, she wrote the same thing, and then she filled in the empty slots with $\mathrm{Z}, \mathrm{Y}, \mathrm{X}, \mathrm{A}$, and $\mathrm{B}$, giving her password EZEYEXAB. She attempted to see what was wrong, but the example she examined did not give insight into how the overcounting occurred (an example that was overcounted would need at least one additional $\mathrm{E}$ in the other spots). She continued to think about the issue, but after more thought she wondered if, actually, Expression B missed some answers, and she said, "Um, I just, I feel that they're not counting things with this [Expression B]" (in actuality Expression B over 90 million too large).

At this point in the interview, in order to save time, I intervened and told Kristin that Expression B was numerically larger than the first answer, and I asked her to think about why that might be the case. In the underlined lines in the excerpt below, as she discussed the overcounting she initially appealed both to her own intuition and to a rule of counting that she has appealed to before. She failed to explain why the overcounting occurs namely that the solution counts some answers more than once. It was not the case that Expression B considers cases that do not exist, but rather that there are situations that end up in more than one of the cases.

$\mathrm{K}$ : Okay, so it is always the opposite of what I think. Um, the reason why I think they're overcounting. I mean, whenever it's an, it just rubs me the wrong way, because whenever there's an at least 3, or an at most, I just always think there should be more than one way, one case. So I just, I don't think they took into consideration the 3 or the 4 or the 5 or the 6 or the 7 or the 8 . 
E: Okay. So that's what makes you think it undercounts, was because it should be taking into account these multiple cases.

K: Right. Or, since he's over-counting, I would think the reason it's over-counting is because they're considering a bunch of cases that aren't existing with, because they're not thinking in terms of, it could be $3,4,5,6,7$ or 8 .

We see in the exchange below that Kristin was unable to correctly reconcile the reasoning for Expression B being incorrect.

K: So, I don't know, I just always, when I see at least, always think cases, otherwise I know you're counting something wrong...But, so I see where they get the $26^{5}$ filling in the other 5 spots with the 26 ... But, I just don't think they thought about really what happens when you have 4 E's, 5 E's, 6 E's, 7 E's, 8 E's.

She gave the explanation, "I know it's wrong because they didn't break it into cases," but she did not clearly articulate what happened mathematically to have caused the overcounting. This discussion ended our work on the problem.

4.2.1.3.3 - Summary. Much of Kristin's activity and language suggests that when she solves counting problems, she is implementing rules and procedures that she learned in her discrete and probability classes. For instance, her statement "But I always do that second after cases" suggests that 'cases' for her is a strategy that she regularly employs, but she did not articulate cases as a means of partitioning the set of outcomes. Much of what Kristin said suggests a formulaic approach to counting problems, and this was borne out in her work on the Passwords problem. In terms of the model, I thus considered much of her work to be within the relationship between counting processes and formulas/expressions. On occasion, however, she considered examples, which I took to indicate a consideration of the set of outcomes, and her use of examples was noteworthy 
on this problem. In particular, she initially used a prototypical example (involving 1's and 26 's) in order to reason about the problem, and this proved to be useful for her (allowing her to realize she needed the binomial coefficient to choose spots to put the E's). Also, while she did go to a particular example (EZEYEXAB) in considering whether Expression B was over or under-counting, her chosen example did not shed light on what was being over-counted. My interpretation is that she did not utilize examples as effectively as she could have. In spite of the fact that she got the problem wrong initially, however, and that she ultimately was unable to resolve the discrepancy between Expression A and Expression B, there were a couple of occasions in which she used setoriented thinking to her advantage, even if very implicitly. For example, she referred to an option of total-minus-bad as a potential alternative way to approach the problem, and she considered a prototypical example in order to determine that her initial answer was incorrect (and that Expression A was correct). Kristin was ultimately not able to understand the alternative expression put before her and which answer was correct and way. I contend that her overwhelming reliance on processes and/or formulas and not on the set or outcomes might have contributed to her lack of success on the problem.

\subsubsection{4 - Other episodes}

In addition to the more detailed excerpts described above, I will now provide four more excerpts from other students' interviews. These represent scenarios that did not arise in the situations above but that help to paint a more complete picture of overall 
student work on the Password problem. I provide only the background and discussion necessary to situate the episode in question.

4.2.1.4.1 - Brandon's systematic list. In Brandon's work we see that a systematic list provided a particularly useful way in which he could draw upon the set of outcomes. While some students immediately recognized that the way of placing E's in the 8 spots is $\left(\begin{array}{l}8 \\ 3\end{array}\right)$, Brandon did not make that connection right away. Instead, as he started to solve the Password problem, he began to efficiently write out the ways in which exactly 3 E's could be placed in 8 positions (see Figure 30). He organized his work and arrived at 56, which is the same as 8 choose 3 .

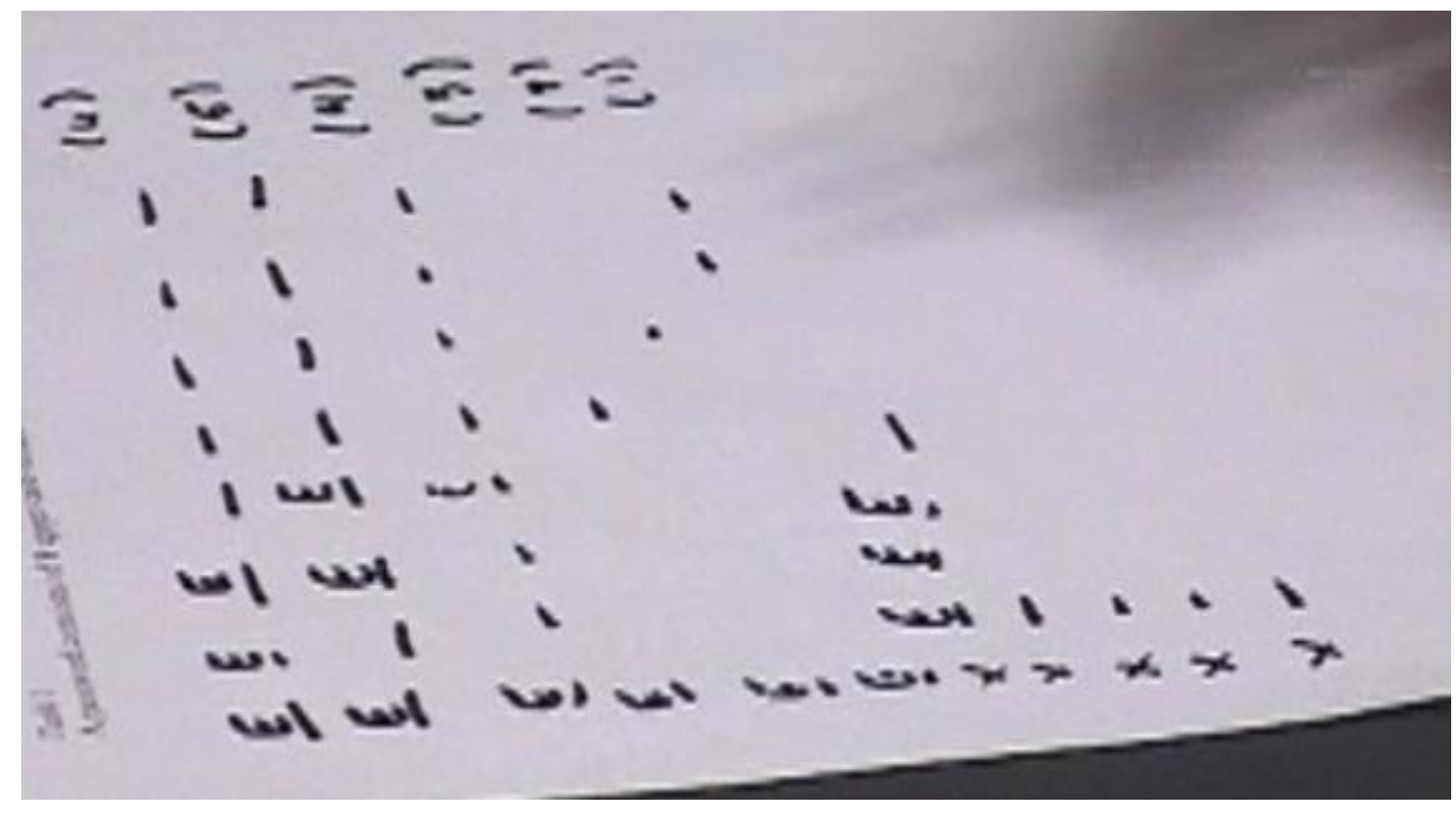

Figure 30

I would describe Brandon's work here as a kind of systematic list. It wasn't an actual list because he did not write the passwords out completely, but he was methodically 
determining the different configurations that could emerge when arranging 3 E's in 8 spots. Although he shorthanded some of the work as he figured out the pattern, his activity of writing out the configurations of E's in the passwords points to a coordination of sets and processes. He was relating his counting activity with some actual outcomes that he could see and work with. He went on to observe that he had choices for the open slots in each case, and in fact he incorrectly determined that 26's should go into each empty slot, and thus his initial answer to the problem was Expression B. In spite of his incorrect decision that there were 26 remaining choices for each spot, the list that he began proved to be useful for him in subsequent work on the problem.

Ultimately, after he spent some time working through the Passwords problem, Brandon was able to see how his initial answer was an overcount. I believe that his initial step of writing out all of the terms helped him later. In the second interview, I had him compare the correct Expression A to his original answer of Expression B, and he was trying to reconcile how he was double counting. He was able to do so, and he referred back to his initial list of the configurations in order to make his argument. When he referred to "these two cases here" in the underlined section below, he was referring to two particular rows he had written down in his initial listing described above. In particular, he looked at the row in which 2 E's were certainly in the first and third spots (and the remaining $\mathrm{E}$ could be in any of the remaining 5 spots after the second $\mathrm{E}$ ), $\underline{\mathrm{E}} \_\underline{\mathrm{E}}$ $\ldots-\ldots$ and the row in which two E's were definitely in the first and fourth spots, but the third E occurred somewhere after the fourth spot, $\underline{E}{ }_{-} \underline{E}{ }_{-}{ }_{-}-$So he had the following two rows written directly on top of each other. 
$\underline{E}-\underline{E}_{-----}$

$\underline{E}_{--} \underline{E}_{-----}$

In the excerpt below, when he says, "let's say I let this character right here have 26," he drew a 26 in the third spot in the second row, giving this:

$\underline{E}_{-} \underline{E}_{-----}$

$\underline{E}_{-} \underline{(26)} \underline{E}-\ldots$

B: I think, so 8 choose 3. I've got my 3 E's, but then I'm, let's just say E E E, these are locked in, these still have 26 different ways they could go...Because, if I'm letting, let's say, let's go back to these two cases here... Where I've got an E in the first one, and then I've got these two E's together, offset by one here and two here. If I let this thing, alright, let's say, let's say I let this character right here have 26, then this, when this is actually equal to E, then it's going to count all of these over again.

Brandon's work here demonstrates a way in which this systematic kind of listing served to help him in the problem. Specifically, the listing accomplished two things. First, it gave him a relatively solid sense of the kinds of objects he was dealing with; the action of writing out the arrangements of the E's made him more familiar with how the E's were distributed in the password than just considering $\left(\begin{array}{l}8 \\ 3\end{array}\right)$. Second, the listing gave him something concrete to which he could refer later during his work on the problem. I conjecture that the fact that he had these prototypical configurations of E's among the letters in front of him helped him to determine efficiently how the overcounting occurred. It certainly could be the case that other students may have similar kinds of concrete objects serve a similar purpose that are not related to set-oriented thinking, but in 
Brandon's case the listing was doubly useful. For Brandon, his listing was a particular way in which he considered the set of outcomes, and it helped him go beyond only considering the relationship between counting processes and formulas/expressions.

4.2.1.4.2 - Marcus' "hypotheticals". In his work on the Password problem, Marcus introduced an interesting new bit of terminology, which I discuss below.

M: ... I'm trying to think how I would explain the, I know I'm re-counting but I don't know how to explain that I'm recounting. Um.

E: Good, and I'll just let you think about that for a minute or two - this is great.

M: Yeah I'll think about it. I guess you could do hypotheticals, where you have what am I trying to show here? That, ah, oh, so, say, in one hypothetical my 8 choose 3 E's all appeared in the beginning, I have 3 E's in the beginning. And let's say my $4^{\text {th }}$ letter just so happened to be an E, since I included that in my answer... That's possible within the realms of my answer. And we'll just call the last $4 \mathrm{ABCD}$.

He had initially gotten the problem incorrect (he originally arrived at Expression B), and I had given him Expression A to examine. While he made sense of what reasoning might be behind Expression A, he suspected that his answer "re-counted" a number of entries, and he was not immediately sure which was right and which was wrong (or if they were the same). In the first underlined excerpt above, Marcus introduced a term, "hypotheticals," and used this notion to address the overcounting issue. As he engaged in the discussion written above, he wrote $\underline{E} \underline{E} \underline{E}$ _ and then filled in the last spot to be $\mathrm{E}$, giving him $\underline{\mathrm{E}} \underline{\mathrm{E}} \underline{\mathrm{E}} \underline{\mathrm{E}}$, and then filled in the last 4 digits with $\mathrm{A}, \mathrm{B}, \mathrm{C}$, and $\mathrm{D}$, giving him $\underline{\mathrm{E}}$ $\underline{\mathrm{E}} \underline{\mathrm{E}} \underline{\mathrm{E}} \underline{\mathrm{A}} \underline{\mathrm{B}} \underline{\mathrm{C}} \underline{\mathrm{D}}$. Similarly, as he spoke in the excerpt below, he wrote $\underline{\mathrm{E}} \underline{\mathrm{E}}-\underline{\mathrm{E}} \underline{\mathrm{A}} \underline{\mathrm{B}} \underline{\mathrm{C}} \underline{\mathrm{D}}$ and noted that he could fill the $\mathrm{E}$ in the missing spot, yielding $\underline{\mathrm{E}} \underline{\mathrm{E}} \underline{\mathrm{E}} \underline{\mathrm{E}} \underline{\mathrm{A}} \underline{\mathrm{B}} \underline{\mathrm{C}} \underline{\mathrm{D}}$ again. 
His idea of coming up with a particular instance thus allowed him to identify and articulate the overcounting that occurred.

M: Or can well call, yeah, we can call the last 4 ABCD. So now let's take another hypothetical, what if my uh, fixed E's, my 8 choose 3 E's were the first 2, skip 1 and the last one? And my last 4 are the same, um, it's still within the realm of this answer to have this one be an E, 'cause I didn't put restrictions on it, I didn't say $25^{5}$, I said, you know there weren't restrictions on it. So, these 2 are exactly the same, which is why I'm re-counting.

I subsequently asked him more about his usage of the word "hypotheticals" (a word I had not heard in this context before), and he gave the following insightful discussion about his understanding of that term and how it helped him work through the problem. In the first underlined excerpt below, he clearly referred to a set of elements and could articulate the larger set of objects he tried to count. In the second underlined section, we see that although he could not conceptualize the entire set at once, he could use an idea of hypotheticals to consider one instance of a password.

E: And you had mentioned, so you were thinking about how you could show how your answer recounts some things. And so you said something about hypotheticals, and that you could write down a couple hypotheticals. And so what do you mean by that? And what does that hypothetical mean to you?

M: Um, to me this is, uh, to me the problem is referring to a set of elements, Where my elements have the form of an 8-digit, or an 8-character, um, password with at least 3 capital E's. So, I know that this isn't an infinite set, I know that there's got to be some limit, but even though I can't conceptualize it, I know that it's probably too big to count, so by hypotheticals I mean just one instance. This password, if you really wanted to, you could have this be your password... Um, and you, you could say that your, uh, fixed E's were the first 3 like I said. You could also make your password a different way, with having your fixed E's be here, but you could consequently wind up with the same exact password because these two could be anything. 
Marcus used his understanding of the set of outcomes and of hypotheticals in order to articulate how overcounting occurred. In particular, he realized that his original answer counted a certain password (EEEEABCD) as two different passwords, even though it should just be considered as one particular password. His work on the Passwords problem indicates a coordination of counting processes and the set of outcomes, and this coordination helped him explain a discrepancy between two answers.

4.2.1.4.3 - Aiden's articulation of a sample space. Aiden provided an example in which a student made articulate statements about the set of outcomes, but my interpretation is that this articulation did not ultimately help him as he revisited the problem. I raise this to point out that while students might have shown some indication of considering the set of outcomes, this did not ensure that they were able to make sense of an incorrect answer.

Below we see that Aiden gave a thorough description of the space of objects he was considering. In fact, this was the most explicitly defined space of outcomes that any of the students articulated. Aiden had initially taken a total-minus-bad approach, and he initially got the answer correct. Toward the end of his work on the problem, I asked him about how he thought about the total-minus-bad that he had done. He then proceeded to put forth a detailed articulation of how he was envisioning the problem and the space of passwords with which he was dealing. It is noteworthy that at the end he noted that while he could consider the set of outcomes in its entirety, he said that he did not consider a particular password in his work.

A: So in this case we have a space of possible passwords, so we have - we have 26 possible values for each element, so we have a space with 26 to the 8 points in it. 
Now, with respect to that space, there's going to be a subset of that space that has the character - the property that we're looking for. So I'm thinking about the - the complement set-wise relative to that big space.

E: Okay. And that's kind of - do you have that picture in your mind?

A: Yeah, that - that's usually what I start with. I just - I go with the entire set, and then I'm trying to kind of find the sizes - - of little pieces of it... So we have this space is going to have 26 to the 8 points in it. And each point, there's going to be a password. So I just imagine this is like - this is my bubble, and then each of these points corresponds to an 8-character password...There's another way we can do this. We can also have - we have 8 components. So we also - this space is actually, if you want to think about it more orderly, it actually has 8 different dimensions. So we have 8 mutually perpendicular axes in here. It's hard to tell, but just imagine there's 8 mutually perpendicular directions in there, and along each of those axes you go from the first character to the $26^{\text {th }}$ character. So I kind of imagine like if - if I think of this as a vector space it's like this discrete lattice that is this 8-dimensional cube, and each point in that cube corresponds to one of those passwords.

E: Okay. Awesome. And then you're organizing them according to - you want the ones that have - well, in this case the ones that have zero, one or two E's in them.

A: Correct.

E: And then those are what you're subtracting out.

A: Exactly, yeah.

E: Okay. Great. And at any time - are you - did you think of any particular passwords? Any particular string of letters that was -

A: Oh, you mean, like a word?

E: Yeah, like a word.

A: Uh, no.

Aiden was successful in his initial work on the problem, and he gave the articulation above about the set of outcomes he considered. However, when we revisited the problem, and I gave him Expression B to compare with his answer, he was unsure of how to reconcile the numerical difference between the two expressions, and he was ultimately not able to articulate clearly how exactly overcounting in Expression B occurred. I highlight this example because it shows that while Aiden seemed to have a clear grasp of 
his overall set of outcomes, and while he used precise mathematical language to talk about his work, he did not use his knowledge to make sense of the issue of overcounting. Thus it does not seem that a clear sense of the solution space is sufficient for a student to successfully identify issues of overcounting. It may be the case that the subspace knowledge is just one facet of a well-developed and complete understanding of the set of outcomes, and that Aiden's incomplete sense of the set of outcomes made him unable to make sense of the overcounting.

4.2.1.4.4 - Daniel's set-theoretic language. I highlight Daniel's work on the Password problem because of his explicit, well-articulated set-theoretic language. Daniel used the notion of partition as he evaluated an alternative solution. In his work on the Passwords problem, he had arrived at a solution of $\left(\begin{array}{l}8 \\ 3\end{array}\right) \cdot 26^{5}$. When we revisited the problem, I gave him the correct answer

$$
\left(\begin{array}{l}
8 \\
3
\end{array}\right) \cdot 25^{5}+\left(\begin{array}{l}
8 \\
4
\end{array}\right) \cdot 25^{4}+\left(\begin{array}{l}
8 \\
5
\end{array}\right) \cdot 25^{3}+\left(\begin{array}{l}
8 \\
6
\end{array}\right) \cdot 25^{2}+\left(\begin{array}{l}
8 \\
7
\end{array}\right) \cdot 25^{1}+\left(\begin{array}{l}
8 \\
8
\end{array}\right) \cdot 25^{0}
$$

and asked him to make sense of it. The set-theoretic language Daniel used was not common for the students. The excerpt below suggests that Daniel's understanding of the notion of a partition allowed him to make sense of the alternative solution he was given.

D: So, uh, obviously you can choose, uh, if you, again you know kind of partition the set of all possible combinations, um, and then out of those count how many are, um, the ones that you want, right.

A bit later he made the following statements, which again underscore Daniel's formal set-theoretic language. 
D: So this one

$$
\left.\left(\begin{array}{l}
8 \\
3
\end{array}\right) \cdot 25^{5}+\left(\begin{array}{l}
8 \\
4
\end{array}\right) \cdot 25^{4}+\left(\begin{array}{l}
8 \\
5
\end{array}\right) \cdot 25^{3}+\left(\begin{array}{l}
8 \\
6
\end{array}\right) \cdot 25^{2}+\left(\begin{array}{l}
8 \\
7
\end{array}\right) \cdot 25^{1}+\left(\begin{array}{l}
8 \\
8
\end{array}\right) \cdot 25^{0}\right)
$$

is, uh, basically the same as I was doing in the other case. You partition the combinations by the, uh, count of, um, by the count of E's, and then, um, because each solution, yeah, then each solution, or what do I want to say, each of these ways of counting only counts the thing in one partition - or what, in one set, or in one element of the partition, I guess.

E: Sure, okay.

D: So, uh, there's no overlaps between them... So if you count all the cases, and you count everything in each case, then you don't have any overlaps and then you don't have anything missing.

Thus, Daniel gave an unusual example of a student who could comfortably use settheoretic language.

\subsubsection{5 - Overall Summary of the Passwords problem}

In the discussion of the Passwords problem above, we saw that in addition to utilizing a case breakdown, some students also gave clear articulations of the total set of outcomes (such as Aiden's description of the sample space). This was far less typical, however, and only 6 other students actually made explicit statements about the set of outcomes as Aiden did. Additionally, when students evaluated the alternative solutions presented to them on the Passwords problem, there were 7 instances in which students suspected that the two different expressions were actually the same, and 4 of those times they were able to reconcile the difference. When this happened, students were typically focused on the relationship between counting processes and formulas/expressions, but not on sets of outcomes. We also saw that students looked to particular outcomes in a variety of ways. While Peter, Kristin, and Anderson did not think of specific passwords in their initial 
work on the problem, they each considered particular passwords in different ways in their revisiting of the problem. Specifically, as Peter revisited the problem, he was able to come up with a particular outcome that was overcounted; before he identified a password that was overcounted by Expression B, he had not been able to articulate why Expression B might be incorrect. Anderson, too, utilized a particular instance to explain the discrepancy between problems, but this came about as the result of a small case. We also saw the importance of systematic listing (even an incomplete listing) in Brandon's solution, and we saw that Marcus utilized "hypotheticals" in his work, for his benefit. From the discussion of the passwords problem, it seems that students' uses of particular outcomes were a relevant aspect of their activity. Thus, we have seen that the Passwords problem was a very rich counting exercise for students, leading to a variety of strategies and techniques, including a number of activities that related to sets of outcomes in particular. This concludes the description of student work on the Passwords problem, and we now move on to a discussion the Cards problem.

\subsection{2 - The Cards Problem}

The Cards problem states, "How many ways are there to pick two different cards from a standard 52-card deck such that the first card is a face card and the second card is a heart?" The most common correct answer to the problem involves a case breakdown of $9 \cdot 13+3 \cdot 12$, and a potential incorrect answer is $12 \cdot 13$. In this section, I will discuss three students' work in detail, and I will also address an interesting feature of one additional student's work on the problem. All 22 students attempted the Cards problem, 
and I revisited the problem with 11 of them. Of the initial responses, 13 got the problem right; 12 of them got the answer $9 \cdot 13+3 \cdot 12$, and one gave a correct answer of $\frac{3}{4}(12 \cdot 13)+\frac{1}{4}(12 \cdot 12)$. One student gave the incorrect answer $12 \cdot 13$, while 8 students gave some other incorrect answer.

\subsubsection{1 - Brandon's work on the Cards problem}

4.2.2.1.1 - Overall description. On the Cards problem, Brandon first drew out some slots, and by considering the number of choices he had for face cards (12) and for hearts (13), he initially got an answer of $12 \cdot 13$. He felt that it seemed too easy, but he was not sure what else to do on the problem. I then intervened and asked him if he could provide some specific examples of what he was trying to count. This proved to be meaningful for him, and as he started to write out an example, he realized that his initial answer was incorrect. In particular, he recognized that the face cards that were also hearts would be an issue in his original answer. He decided to break the problem down into cases and ultimately arrived at the correct answer. In our discussion of the problem, he used language that suggested some elements of set-oriented thinking.

\subsubsection{2 - Detailed account}

4.2.2.1.2.1 - Brandon arrives at a common incorrect answer. Brandon began by utilizing the slot language, and he described the number of choices he had for each slot. He recognized that there were 12 face cards in the deck, so he had 12 choices for what could 
be in his first slot. He argued that there were 13 hearts that could go with each of the 12 face cards, and thus he got an answer of $12 \cdot 13$.

B: So, what we do first is, again my little underlines for just slots of where we need to pick something. Um, we have face cards, jack queen king, uh, 12, wait, yeah 12 choices of cards that are face cards, out of the 52, so I would say, there's I've got 12 choices here for this first slot... And then for each one of these cases, it's going to correspond with a distinct outcome where we've got 13 hearts, so, let's say I got, I've picked my jack of clubs, then there's 13 different ways that I can pick my, uh, my, my heart. So I would just say 12 times 13 . Is that right? ... Seems like it'd be more complicated than just 12 times 13, but that's all I'm coming up with.

It is interesting to note (in the underlined section above) that Brandon used language that suggested he considered outcomes. Specifically, he noted that for each of the 12 face cards, when paired with one of 13 hearts, it would give a different outcome. This implies that he considered the set of outcomes, although he did not proceed to detect that some outcomes were repeated. Also, the answer seemed too simple to him, and he showed some hesitation and lack of confidence in his answer. Brandon's work to this point was thus not an instance in which he looked only to counting processes and formulas/expressions with no consideration of outcomes; he considered outcomes, but he did not detect any problematic ones at this point in his work.

4.2.2.1.2.2 - I prompt Brandon to provide an example of what he is trying to count, which proves to be productive for him. I then asked Brandon if he had pictured anything as he thought about the problem, and he said that he considered actual physical cards and 
thought about getting a face card and a heart ${ }^{32}$. I then intervened by asking him to provide some examples of what he was trying to count. My rationale for this prompt was that I was curious whether considering a particular example of what was being counted might help him notice that his answer was incorrect (and particularly that his process had overcounted in some way). As the excerpt below shows, as he started to articulate an example of what he started to count, he made a realization about his work - a literal "aha" moment.

E: Okay so one question I have, can you give me like I don't know 3 examples or something of, 3 specific examples of what you're trying to count?

B: How do you mean?

E: Uh so like, what's, what's going to be, what's a possibility of what you're, what you're counting in this problem?

B: Just like, uh, if we say if we get the first card first and let's say it's jack of hearts [writes J and a heart] - oh! Aha I think I've stumbled onto something here.

E: Okay.

B: Um, 'cause say if I think it just got more complicated, where let's say that first card, let's assume it's not a heart, So, case 1, so this is, I found out why that's wrong.

Brandon thus found out what was wrong, and he described in the excerpt below how he could fix the issue. When he referred to "case 1" and "case 2" below, he broke up the choices for face cards initially into two distinct scenarios - whether or not the first card is a heart. His work below is characterized by slots and choices language, and his case

\footnotetext{
${ }^{32}$ This problem tended to evoke the most visual imagery among the students, who were familiar with cards and could visualize actual cards. A number of students remarked that they visualized cards as they tried to think of how many face cards there were. However, it is not clear that on the whole, when visualizing the cards, the students considered what they were trying to count, namely pairs of cards where the first is a face card and the second is a heart.
} 
breakdown suggests that he broke the outcomes down into two different kinds - those in which the first card (a face card) is or is not a heart.

B: Case 1, uh, a face card not a heart, so there we've got 3 times, 9 different choices there for my face card. And then we haven't depleted the total number of choices for hearts, so that would still be 13, so that's case 1. Case 2, a face card is a heart, we've got 3 choices there, jack, queen, king of hearts, one of those 3, and then we have depleted, we have depleted the total number of hearts now by 1 , so that's 3 times 12, and then you would, these are distinct, uh, distinct cases so you would add 9,13 plus 3 times 12 to get the total number of different hands that you could get, where, uh, one is a face card and the other is a heart.

When asked to reflect upon what he saw to change his answer, Brandon explained that he was trying to make sense of why his initial answer may have been "too simple."

E: Okay, cool, and so can you explain that, you had kind of an aha moment of starting to write down jack of hearts and then changed your answer. So what, what were you thinking about, what happened there?

B: I uh, I'm not sure, I just kind of, just kind of realized that, I don't know for some reason I just wrote down, I was trying to tease out why this is too simple of a solution, and so maybe just trying to get a, a, a solution that might gum up the works a little bit there,... And so, for some reason, uh, a face card that is a heart seems to be the logical way to go there.

In the underlined text above, he had looked to identify "a solution" that might be problematic for his initial answer. By "a solution" I interpret that he meant a particular outcome of his counting process. He did not attribute this realization to the prompt I had given him, but the focus on articulating an instance of what he was counting seemed to have put his productive line of reasoning into motion.

4.2.2.1.3 - Summary. Brandon first arrived at an incorrect answer of $12 \cdot 13$, and while he did not feel extremely confident in his answer, he could not articulate how it might 
have been incorrect. He did not, on his own, carefully articulate instances of what was being counted. However, when I prompted him to write down particular examples of what he was trying to count, this triggered for him something that he needed to fix about his answer; he realized that there may be a possibility (where the first card is a face card and a heart) that might have caused problems in his original answer. After he recognized the potentially problematic pairs of cards, he was ultimately able to arrive at the correct answer. In terms of the model, Brandon benefitted greatly by being asked to explicitly consider some elements of the set of outcomes and relate it to what he was doing on the problem. This is reflected in the relationship between counting processes and sets of outcomes.

\subsubsection{2 - Peter's work on the Cards problem}

4.2.2.2.1 - Overall description. As Peter first oriented himself to the problem, he addressed the issue of order, recognizing that there was a first card and a second card. In his work he considered the number of possibilities for the first card and the second card, and this led him to an incorrect answer of $12 \cdot[12+13]$. I asked him if there was a diagram he could articulate, and he drew a diagram which ultimately proved to be quite useful for his progression on the problem. While the diagram did not immediately alert him of his error, as he subsequently discussed his work and referred back to the diagram, he realized his mistake and ultimately arrived at the correct answer of $3 \cdot 12+9 \cdot 13$. Also, I had asked him to write down some particular instances of what he was counting, and he did this, although it did not seem to help him advance in his thinking about the problem. 


\subsubsection{2 - Detailed account}

4.2.2.2.2.1 - Peter initially arrives at an incorrect answer. As Peter began his work on the problem and started to make sense of what he had to do, he made a comment about the order of what he was trying to count. As we see in the underlined sections below, he paid particular attention to the fact that the order was relevant in the pairs of cards.

P: Um, I know that there's 13 hearts, and I've got to count for the face cards. Let's see there's, Jacks and, there's 3 times 4, so there should be 12 face cards. Okay so there's 12 face cards, um, and then there's, uh, 13 hearts. That's just because it's, uh, 52 divided by 4 . Um, so okay, um, and I'm picking 2 different cards, um, if I didn't really care what the cards were, um, I could pick 2 cards from 52, but we care about the order of them, right, so, what I want to say is, um, I can have 52 cards to choose from for the first one, and then I have 51 different cards to choose from the second one, that's if I don't care about some, what they are. That's, uh, just choose 2, cards in order, I guess. Um, so okay.

E: And when you say order matters, like choose 2 cards in order, what do you mean by that?

P: What that means is like, um, if I get a 2 and 3 that's different from getting a 3 and a 2.

While the example he gave was not specific to the problem (he referred to two outcomes that are not related to the problem at hand), I interpret that his reference to a particular example suggested that he evaluated whether order mattered based on whether some outcomes were considered the same or different. In the underlined text above, even though he talked about having 52 choices and 51 choices (which is not correct), I emphasize his discussion of order; he would later correct the number of choices he had at each stage.

As Peter continued his work, he recognized that there were different situations he'd need to deal with, and he reasoned that he should implement different cases. He saw that 
he would have two different situations depending on whether or not he got a heart as the first card. Note, too, that in his description he spoke of first picking a card, and then splitting into two cases; this would be reflected in his answer and will be discussed subsequently.

P: Okay well there's a couple different, uh, situations here, because we can have some hearts that are - or face cards that are also hearts. Um, so the way I think about it is, (I kind of remember that there was a faster way to look at it), but the way I'm thinking about it right now is that, I'm going to go ahead and pick a card, and I want to see how many face cards I can get, and then I'm going to sort of split that up into 2 cases. One of them is if I got a heart in the first one, and the other one is if I didn't.

Having decided that he wanted to use two cases, he investigated what those cases might be. During the excerpt below, he wrote the following. As he said, "Okay so I can have 12 face cards for my first one," he wrote $12 \cdot[\quad]$, and he began to think about what might fit in the blank. Ultimately he arrived at $12 \cdot[12+13]$. This answer suggests what he had said above, that he would first pick a card and then split into his cases.

P: Um, so okay, so I have 12 possible face cards, so there's 12 ways that I can draw a face card [draws $12 \cdot[\quad]$ ]... However if I, the second one is, like I said we've got these two different cases, so I'm going to have to sort of add the number of ways for those two different cases. Um, in the one case I, um, got a heart, I guess maybe I'll say it's a face-heart. And the other case I'll say it's um, I got a nonheart face, I guess. ... Okay um, so okay if I got a, uh, heart face card, then that would mean there's only 12 hearts left, because I had 13 to begin with... So I've got 12 left to pick from, so I can pick any of those 12 [draws $12 \cdot[12+]$ ]. And if I, uh, however I didn't then I should have 13, so there should be 13 left there [draws $12 \cdot[12+13]]$. Um, actually I don't think that's right, though. 
Peter then went through a short period in which he questioned his work. He felt that his $12 \cdot[12+13]$, which would equal $12 \cdot 25$, or $12 \cdot 12+12 \cdot 13$, might be incorrect, particularly because he did not feel that he should have 25 choices for the second card. He continued to think about the problem, but as he talked through it again and described the process by which he had arrived at his expression, he convinced himself more fully that he believed his answer was correct. We see below that it seemed to be the fact that he could think about the answer $12 \cdot 12+12 \cdot 13$ as two different cases made him think it was correct.

P: Um, I think these just represent different cases. I mean just different situations, like I'm thinking all the different ways that I can do it, I can do it in this situation or I can do it in that situation. So in this case I've got 12 and then 12, and in this case I've got 12 and then a full 13, so I, I guess it is, I think it is correct. So I think it's 12 times 25 , I think that's the answer.

I asked him to expound a bit more on what the first 12 represented to him, and he responded with the following discussion about splitting into "two universes." This gives some insight into his understanding of what the case breakdown accomplished for him, as he was able to articulate what he thought the different cases should entail. It is also noteworthy to see that, again, he split into the two universes after he made the initial choice of face cards for the first card (highlighted in the underlined section below).

P: So, like I said I think there's, Jack, King, and Queen, and there's 4 different suits, so uh, 3 times 4 is 12 face cards so I can grab any one of those 12 , that means there's 12 ways to do that, um, and then, I'm kind of, I split it into two universes, in one universe I got a heart on that one, and in that case I've only got 12 hearts left out of the deck to grab from...So I can do that in 12 ways. In the other universe, um, I didn't get a heart, and so there's still 13 hearts in the deck, and I can grab any one of those 13 . And I'm thinking that I, so I, I, sort of add that, the 
first 12 times 12 to the 12 times 13, and that should be the total number of ways that I can, that I can get a first card face card and second card being a heart.

To this point in the interview, while Peter had at times seemed to consider sets of outcomes as he counted, he had not seen the error in his answer. Indeed, he seemed to rely heavily on the counting process that yielded his expression as a means by which to check the reasonability of work.

4.2.2.2.2.2 - Peter draws a meaningful and useful diagram. I then asked Peter whether there was a picture or diagram that he could draw. This was becoming more of a standard question in the second stage of interviews, and my motivation was to see if a diagram might help him recognize the error he had made. He drew a diagram (Figure 31) which would serve him well. In particular, he drew 4 circles, which represented the four different suits of face cards. Then, noting that one of those groups would be hearts, he drew a longer shape that included one of the circles as a subset, and he labeled that "hearts." Thus his diagram showed the relationship between hearts and face cards in a standard deck of cards. 


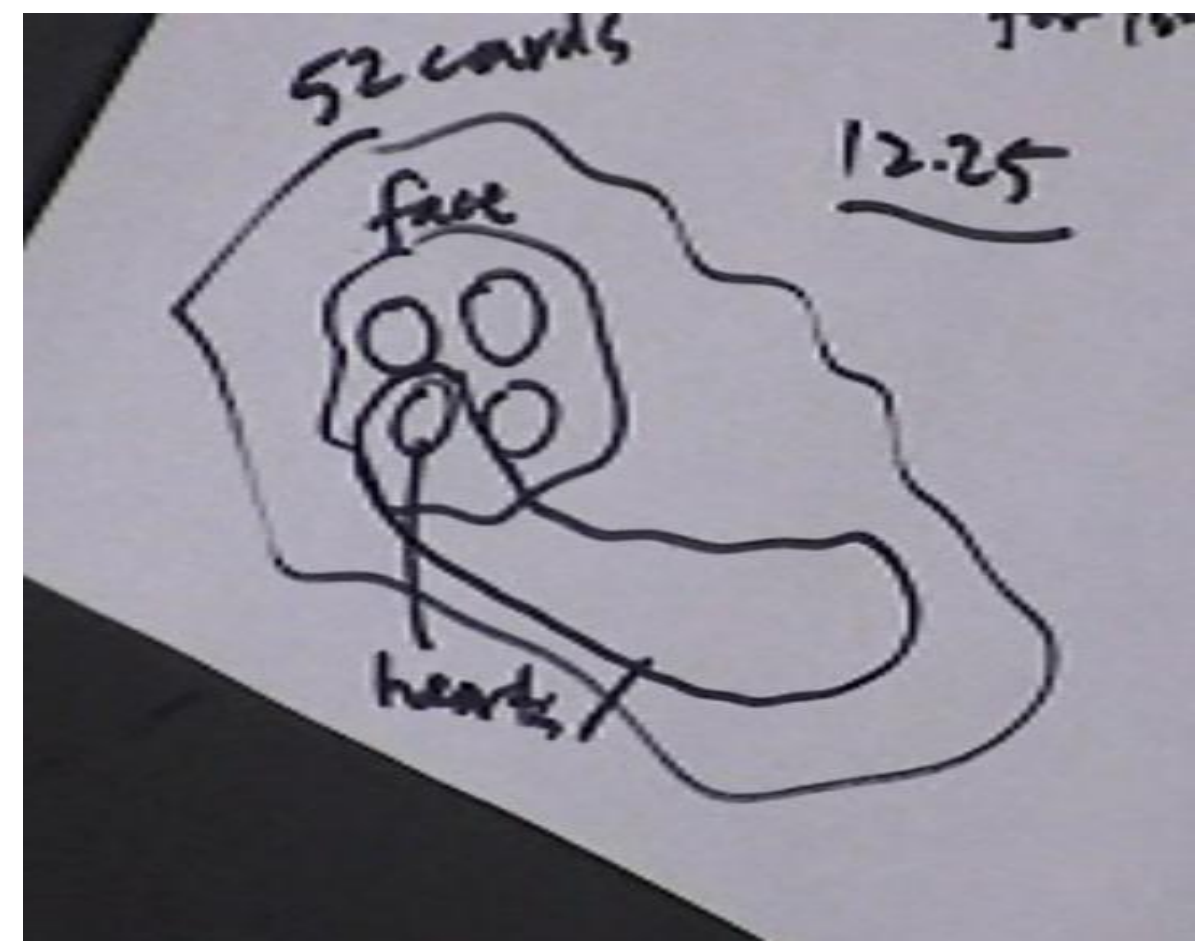

Figure 31

At that point in his work, drawing the diagram did not make him change his answer. That is, even after drawing the diagram, he stuck with $12 \cdot[12+13]$ and did not see anything that he should change about his initial answer. I thus intervened and asked him to write examples of what he was trying to count, and this was an instance in which the prompt was not very effective.

E: Can you do me a favor and, within, um, you had mentioned two scenarios. You know where you get a heart maybe first, or where you don't get a heart first, I mean, that's a face card.

P: Right, right.

E: So can you, like, draw, or write a couple of examples of the things that you're 
trying to count, maybe in each of those scenarios, so like, like what are you, what is it that you're trying to count, and can you just tell me an example of what you're trying to count?

P: Okay, I'm not sure if I understand the question completely. What you're saying is, where do I get these numbers kind of?

E: Well not even where you get these numbers, but just, like what's one instance of something that's, that you're counting.

P: Okay like an example of, okay so for example I got a jack of hearts and then I got a, you know, a 2 of hearts or something.

E: Yeah, yeah, so something, so that's one example. Can you, um, yeah give me maybe an example that's in, that's in each of your two scenarios, like,

P: Oh okay so one from each scenario? Okay so, like in, okay I see. In the first scenario I got, uh let's see, the first one is that I got a heart, right? So that would be like the Jack of hearts, um, and then like say the 3 of hearts or whatever. And, um, another one would be, let's say I got instead I got the jack of clubs and then I got say the 3 of hearts. Um, okay, I guess. So yeah, this, this one would be in, uh, the case where I got sort of like, this is like 2 hearts, but one of them happens to be a face, and then this is only one heart. Is that kind of what you were asking?

Peter's work in the excerpt above stands in contrast to Brandon's work on this problem, in which the prompt for him to write down a particular instance made him adjust his answer. As we see below, Peter did give examples, but they were not problematic examples, and the activity of articulating particular instances did not make him reevaluate his answer.

Then, Peter started to talk back through what he had done. He noted that he had not actually been thinking in the kind of specifics that I had just asked for (such as particular instances of pairs of cards), and he tried to explain what he had been thinking about. In doing this, he referred explicitly back to the diagram he had drawn (Figure 31). In fact, his drawing of the 4 circles was quite important, and I believe it was this that allowed him to see that he needed to divide up the 12 choices for face cards into 3 and the 9 . Below, 
when he said, "I'm grabbing from these 12 " he pointed to the 4 circles he had drawn, 3 of which are not included in the set of hearts. He realized that "there's only 9 here that aren't included in this," and the 9 are those 3 circles of 3 cards that are not also hearts.

P: My numbers here, I've still got 13, I mean I'm grabbing from these 12, but there's only 9 here that aren't included in this, right [points to the four circles representing the face cards], so if I grab from one of these [points to the three circles not in the heart subset], um, oh, yeah, maybe that's the key actually. Maybe I did analyze it wrong. Maybe I, uh, maybe I should have analyzed it kind of thinking of these two universes from the very beginning instead of splitting off at the choice.

E: Okay. Well yeah, so keep telling me about that.

P: Um, what I'm just thinking now is that maybe, um, really what I'm thinking about is, I really need to add two different situations, 'cause in the first problem, I actually have to draw a non-heart face, not any face, so this number should be too big for the second case. So I think it's 12 times 12 plus 9 times 13 is I think is what I've got actually.

It is also key to see (underlined above) that he stated that he needed to split into "these two universes" initially instead of once the first card had already been chosen. This was an insightful realization, and it allowed him to address the reason for his incorrect answer. As he described his work in the excerpt below, he realized the issue, but he did not completely resolve it. He realized that the non-heart face cards should be 9 options instead of 12 , but he kept the number of heart face cards at 12 instead of reducing it to 3 (my interpretation is that this was just a numerical/computational mistake on his part, one that he would subsequently fix). I then asked Peter to discuss that new answer of $12 \cdot 12+9 \cdot 13$ he had just proposed. As he talked back through it, he drew another informative diagram (Figure 32) to explain the difference between his first approach and his second approach. I will discuss the excerpt below. 


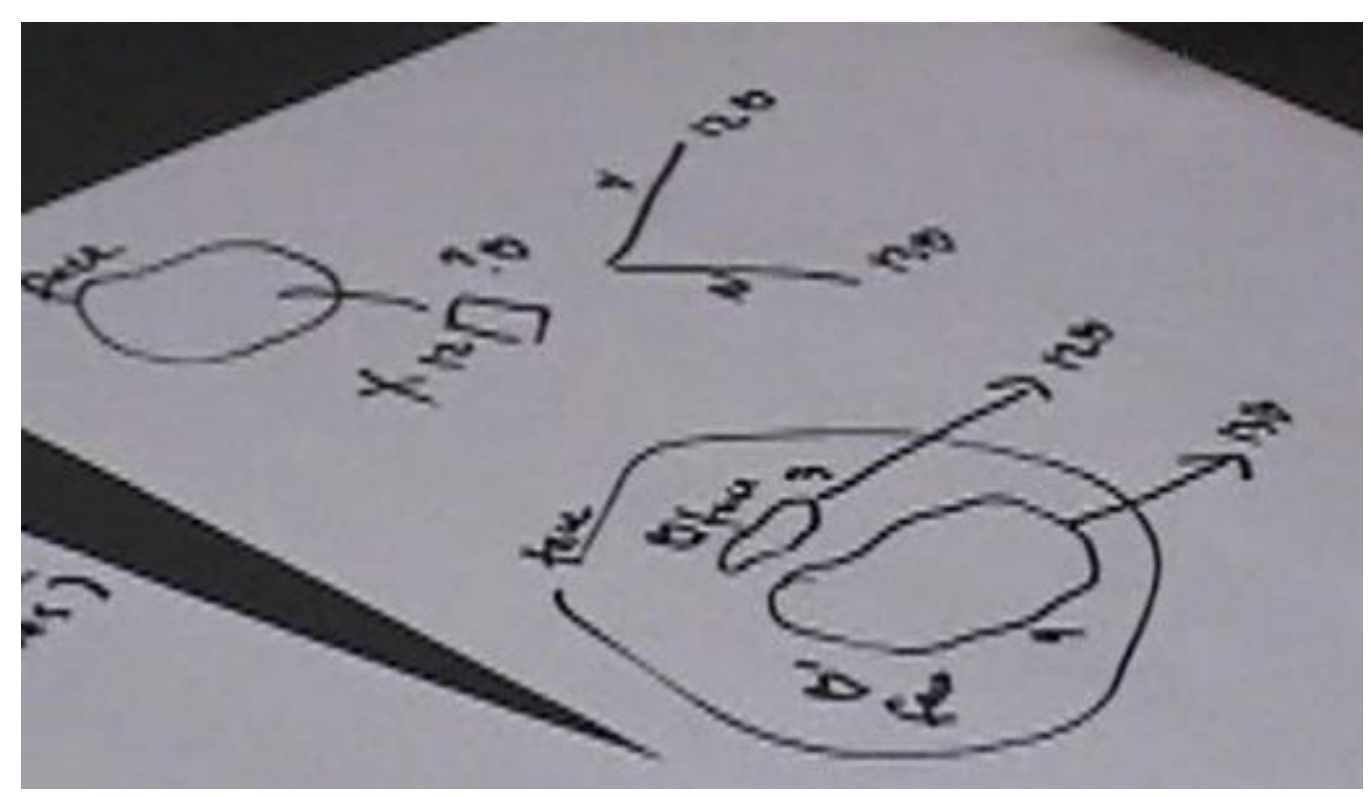

Figure 32

P: So actually it should be, I should have split it up from the beginning. What I should have is the, sort of the heart faces in this, and then sort of the, let's say the heart prime faces (laughs), the non-heart faces in this group. These are all the face group there... There's 3 here and there's 9 here. In this case, I grab one in 3 ways, and then I know I have a heart, so I've got 12 hearts left to draw from. In this case I draw 1 in 9 ways, and, um, I know I have 13 hearts left. And um, yeah. At least I think that was my error, was making the choice here rather than up here... So I think that the right answer is 3 times 12 plus 9 times 13 .

In his first attempt, he had considered all of the face cards at once, as a set of 12, and then for those 12, he had 12 and 13 choices respectively if that first card had or had not been a heart. He began by drawing a circle that represented all 12 of the face cards. And he drew a small tree in which two branches stemmed from this set of 12 , one branch that represented having drawn a heart first (leading to a 12), and one that represented having drawn a non-heart first (leading to a 13). This thus represented his $12 \cdot 12+12 \cdot 13$ answer. Below this, he drew a diagram that represented his new answer. He drew a large 
set representing all of the face cards, but within that set he separated out subsets for hearts and non-hearts, respectively. He then split into cases based on whether his first choice was from the subset of heart face cards (size 3) or non-heart face cards (size 9), thus changing his answer to $12 \cdot 3+9 \cdot 12$.

4.2.2.2.3 - Summary. I chose to discuss Peter's episode because I believe it gets at a particular way in which a diagram helped Peter see his error. The first Venn Diagram he drew allowed him to see that he needed cases at all, and writing out the second diagram caused him to get the actual numbers right. We see the value in his consideration of sets and the Venn diagram, and it was talking through his diagram (and the particular way in which he drew it) that enabled him to see how he should fix his error. Also, this example indicates that there was some set-oriented thinking, although the set theory is related to the set of objects that he has to choose from, not the set of outcomes itself. That is, the elements in the Venn diagram he drew were single cards, not pairs of cards. This occurred for other students as well, as set-theoretic language and discussions arose that were separate from the context of the set of outcomes. Seven times students explicitly used set-theoretic language on this problem in order to refer to the sets of objects from which they had to choose. On this problem more than on any of the other problems, students used formal set language in order to talk about their choices. They referred to the set of face cards, for example, or the set of cards that are hearts. I interpret that Peter drew upon all of the relationships and components in the model in his work on the problem. At times he relied heavily on the counting process and the 
formulas/expressions, but he was also able to consider the set of outcomes in order to realize an error in his answer and to ultimately arrive at a correct solution.

\subsubsection{3 - Paige's work on the Cards problem}

4.2.2.3.1 - Overall description. After Paige oriented herself to the problem, she tried counting it directly, but she ran into some problems with what the numbers of choices would be, particularly for her second card. She realized that the choice for the second card might change depending on whether or not a heart was chosen as the first card. I asked about her visualization of the problem, and she drew a Venn diagram that had pairs

of cards as the elements. Then, after I prompted her to write some specific examples of what she was trying to count, she transitioned to an approach in which she subtracted some solutions that had been overcounted. She recognized that she wanted to subtract some pairs that were overcounted, but she subtracted off too many elements and failed to see any error in her work. I then gave her a correct alternative answer, and she was able to explain how a student arrived at that expression. As she numerically compared her answer and the alternative, however, she could not reconcile the difference. After working on the problem for some time, and going back and forth between the answers, she ultimately could not determine which answer was correct and why.

\subsubsection{2 - Detailed account}

4.2.2.3.2.1 - Paige arrives at an incorrect answer, but displays some evidence of a set-oriented perspective. Paige struggled a bit with the problem initially. She first oriented herself to the situation and confirmed the numbers of hearts and face cards in the deck. She 
considered choosing from 52 cards, but she realized that she had 12 options for her first card (as there are 12 face cards in a deck). She tried to determine the number of choices for the second card, but she saw that the situation would get somewhat more complicated because the second card could have also been a heart. We see in her work below that she experienced some trouble finding a solution.

P: But that's, yeah, so I think that's right. And then, so there are 12 ways I could choose that face card, and now I have an "or" situation, because the second card being a heart. I could have picked the heart out, like I could have, like, got the jack of hearts or the queen of hearts or the, and so that's going to cause a little bit of overlap I think in this counting problem. So if I just say 12 times 13 , 'cause there are 13 hearts, Um, I would be overcounting, because there are some situations here, so how many problematic cards do I have?

In particular, as she talked through it, she had written 12 , but she was not sure what to write next. She considered writing $12 \cdot 13$, but she realized that such a solution might have overcounted. In the first underlined bit of text, we see that she considered some particular cards; this indicates that there was at least some consideration of some concrete examples. And, too, she used overlap language as she discussed the overcounting, which suggests perhaps a coordination of sets and processes - that she realized her multiplication of 12 and 13 could have yielded some outcomes that were counted more than once (the second underlined portion of text). This language suggests that she considered the set of outcomes, even if implicitly.

While she realized that 12 times 13 would not work, she was not sure what to write for the second case, and she did not seem sure of how to proceed. I then asked her if she was visualizing anything, and she noted that she was not, and that this was perhaps 
contributing to her difficulty. She indicated that she might want to try a smaller case as she had done on the previous problem, but I intervened with a prompt to try to point her toward considering particular instances of what she was trying to count ${ }^{33}$.

E: Is there any kind of picture or diagram that you're envisioning?

P: Not right now, and that's making it harder... That it's like, just a little bit too big to picture. And I want to jump to a smaller case a little bit, but, well maybe I can do that, actually, maybe that'll help. Because, so my inclination here is saying that like, if all 12 of these are face cards, I want to know how many of these are hearts and not face cards, and then my idea is that if one of them is in this group, then I would multiply this by, um, hmm. Yeah I'm going to go to a smaller case.

E: Well and I wonder if you can, um, can you give like an example or two of the things that you're trying to count?

P: Sure, so, um, I'm look - so I guess the things I'm trying to count or the things that I'm seeing as problematic?

E: Well maybe first the things that you're trying to count. Like what are some things you're trying to count?

P: So like list out my...

E: Well, or yeah, or even just give me a couple of examples.

While Paige had some implicit sense of outcomes, as mentioned above, she had not overtly considered any particularly outcomes. She acknowledged that this was problematic and was hindering her thinking about the problem, so as we proceeded she tried to focus more clearly on the set of outcomes.

4.2.2.3.2.2 - Paige writes some examples and subtracts repeated outcomes. I then asked

Paige to write out some particular examples, and she wrote down three specific pairs, all of which had the king of diamonds as the first card and a non-heart face card as the

\footnotetext{
${ }^{33}$ I intervened here primarily because I felt that developing an isomorphic smaller case might have been too difficult and time consuming to be useful for her, and I was curious about whether listing particular elements could have been useful.
} 
second. After she wrote these, she noted that there might be a problem if she began with the king of hearts (see the underlined portion below). She did not explicitly indicate that the writing of particular examples helped her identify something that might be overcounted, but it seems to be a reasonable possibility that the drawing of the instances (even ones that were not themselves problematic, such as those in which the king of diamonds was the first card) caused her to consider examples that might be problematic.

P: So if the first card is a face card it could be, like, a king, and then I'm looking at hearts, so, it could be the king and then like, king of something that's not a heart, let's say, king of diamonds, and then like a one of hearts, or the king of diamonds - I'm going to start calling that KD, and the 2 of hearts. And then we've got the king of diamonds and the 3 of hearts. So then this would be face cards, um, and then these would be things that are hearts... And so I think a problem that I'm seeing is that if I count like the King of hearts, then it's already taken out, And so then I would only have, like, I couldn't have the king of hearts to do over here.

Paige then considered what might happen if she subtracted bad cases from $12 \cdot 13$. Her subtraction of bad cases is similar to a total-minus-bad strategy because she is taking away undesirable repeated outcomes from a larger number of outcomes (technically she is not removing bad cases, but instead is subtracting cases that have been counted too many times). I interpret this activity as being an instance in which Paige utilized setoriented thinking, because it suggests an ability to organize her outcomes to allow for the subtraction of repeated outcomes. Indeed, her work highlights the relationship between counting processes and sets of outcomes. In the following excerpt, she notes that she thought that there were three face cards that could cause the overcounting (jack of hearts, queen of hearts, and king of hearts), and that for each of these three cards, there would be 12 objects that were overcounted. Thus, she arrived at 36 total pairs of cards that she felt 
needed to be subtracted from the expression $12 \cdot 13$, giving her a total answer of $12 \cdot 13-3 \cdot 12=120$

P: So I guess maybe I could just take out the overlap. I could do 12 times 13 and then take away, and actually that's not so hard to count, is it, to think of like, how many ways could I have jack of hearts for the first card, and then all of the other cards. So, jack of hearts, and then how many cards are left, well there would be, um, 12 hearts left, and then if I picked the queen of hearts first there would be 12 hearts left still, and if I picked the king of hearts first there'd be 12. So if I take away 3 times 12 I think that's going to be right.

I then asked Paige about how she was visualizing the problem, and she drew a Venn diagram to represent what she was thinking. I believe it is noteworthy, though, that she did not completely explain or articulate everything in her diagram, and I think this ended up having a subsequent negative effect on her ability to make sense of an alternative solution. As she drew the diagram, she certainly used set-theoretic language with words like "overlap" and "disjoint," which is seen in the underlined portions below.

E: Okay cool, um, and okay so now, I mean now does any picture pop up, or can you draw any kind of diagram to show what you're counting, or (inaudible).

P: So, I guess if I was thinking of this as a, like, sets, um, and I think, could you hear the words like as I was talking about like overlap,

E: Mm-hmm, Mm-hmm.

P: That like I'm thinking of this as, um, they're not disjoint problems, because I have this case where I have, um, the first card is a, what was it a face card? Yeah. The first card is a face card, the second card is a heart, and so there's this big group of cards whose, like if you pick 2 of them out, the first one be a face, and second, and this is, like sort of in a bigger group of cards where it's just the number of ways you could pick two cards out of everything. So the sample space here would be, um, 52 choose 2,

E: Okay. 
P: Um, and I say choose here because when you choose 2 cards like when you're playing poker or something, the order of the cards that are in your hand doesn't matter. So the, the bigger group that this is a subset of is 52 choose $2^{34}$, that's like all the different ways I could pick 2 cards, and then inside of here, um, I guess this is like, if I didn't, first card is a face card second card is a heart, and maybe this is why I'm having trouble with this problem in general is trying to picture like, I know there's an overlap happening between two groups, you know, and I need to like cut this out,

E: Okay.

P: But I don't know exactly how to formulate with words what that second group is. I guess the second group would maybe be the first card was a heart, like how many different ways can you have the first - oh face card and a heart. So how many cards are there, oh that's what this is. So this is 3, is the number of face cards that are hearts, um, and so that's the group that you're like taking out. Although that's totally a subset of this. Yeah the picture isn't very good in my head. Sorry.

The diagram Paige drew is included below (Figure 33), and it is noteworthy for two primary reasons.

\footnotetext{
${ }^{34}$ The 52 choose 2 is incorrect, in fact there are $52 * 51$ total pairs of cards because the problem distinguishes between a first and a second card.
} 


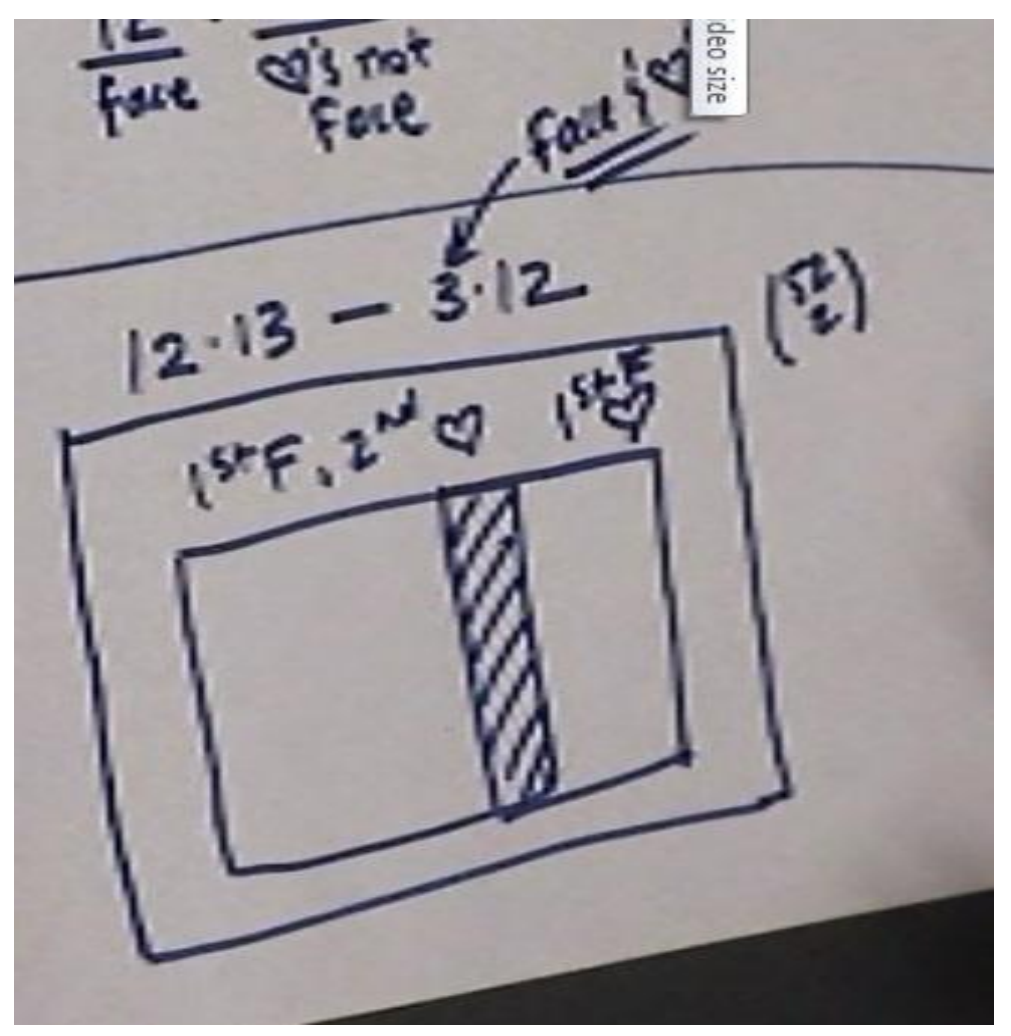

Figure 33

First, we see that the Venn diagram has, as its elements, pairs of cards, and not single cards. This is in contrast to other types of Venn diagrams that students drew for this problem (and indeed to a diagram Paige would later draw) that had single cards as its elements. However, despite having this advantage, Paige's diagram is not correct. The two sets she described ( $1^{\text {st }}$ card face card, second card heart, and first card heart face card, second heart) do not intersect as she drew them (the latter should be a subset of the former). She mentioned something about there being three problematic cards (the face cards that are also hearts), and said, "so this is 3, is the number of face cards that are hearts, um, and so that's the group that you're like taking out." However, Paige never carefully articulated these pairs of cards that were in the overlap, and the usefulness of 
her diagram is unclear. Indeed, it is difficult to conceive of an effective Venn Diagram for this particular problem in which the elements of the sets are pairs of cards and not individual cards.

At the end of her first try at the problem, Paige also made comments that seemed to indicate that she could coordinate counting processes and sets of outcomes. Note in the text below, she talked about a "stage 1" and "stage 2" in her counting procedure, in which her choices for the first and second card represented first and second stages of the counting procedure, respectively.

E: Okay, no no no, that's okay. But there's some sense of overlap, but maybe it's harder, you're having a hard time articulating like what each of those sets should be or something?

P: Mm-hmm, yeah I think so. I think, like, I know I'm overcounting things, because I know they're going to be this cases where like picking a face card that is a heart out first is um, affecting how many hearts are in the second stage of the problem, Like thinking of it as stage 1, stage 2, Um, but I'm having a hard time picturing, like, the sets, like where they overlap, and like, like if I'm thinking about 2 sets being overlapping, is this one just like a proper subset, or is it a piece of a bigger set that's overlapping? Like I guess, I don't know if it's, um, like 2 sets like this and I'm counting up the overlap that way, or if it's like this and I'm just taking this out because that's overcounting.

E: Okay, I see.

P: That's the part I'm having problems with.

She then related this notion of stages to some set of outcomes, suggesting a set-oriented perspective. The underlined parts of the excerpt above highlight this phenomenon. We also see that, although she recognized overlap and saw the need for some subtraction, she was unsure of what kind of subtraction scenario she faced. In fact, in Figure 34 below, she drew two kinds of Venn diagrams representing two situations - one in which the 
intersection between two sets was a proper subset of the other set, and one in which the intersection was not a proper subset.

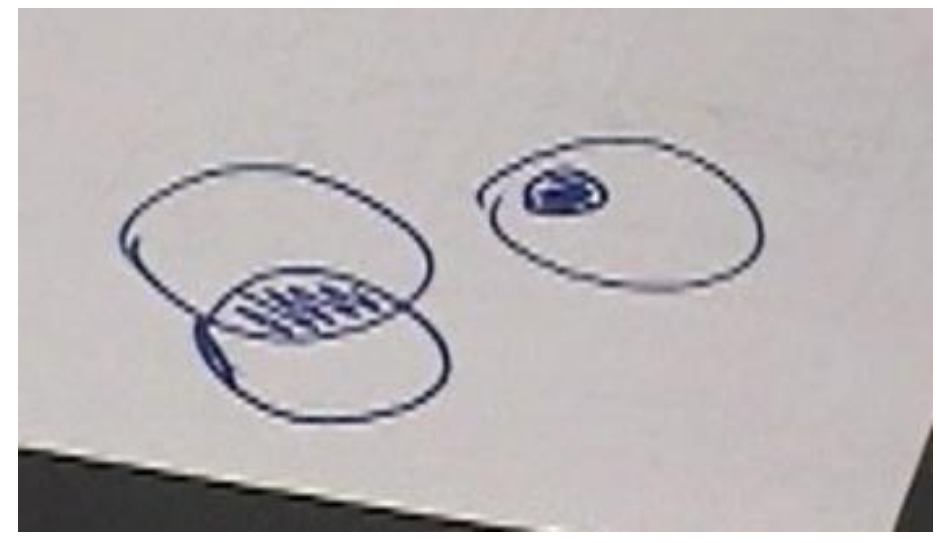

Figure 34

\subsection{3 - Paige was unable to reconcile the differences between her solution and the}

alternative. When we revisited the problem later in the interview, I gave Paige the correct alternative answer of $9 \cdot 13+3 \cdot 12$. She made sense of it, and then she went right to a numerical check to see if her answer matched the new one. Her initial answer, $12 \cdot 13-3 \cdot 12$, yielded 120 , while the alternative gave 153 . For quite some time she compared the two, and she seemed sure that her own answer was correct and that the alternative had been overcounting. She went back and forth for several minutes, trying to decide which was wrong, and why. I include the excerpt below in order to get a sense of the back and forth she experienced in thinking through this problem.

P: And, do I need to, 12 times 13 is what? $156-36$ is 120 . And they've got 9 times 13 , is that 117 , yeah, and then 3 times 12 is 36 . Oh yeah so we're getting - did I do that right? 156 minus 36, yeah that's 120. And then they're getting a much bigger answer, so, my answer was 120 , and they're getting 153 . So why are they getting they're overcounting again, right? So why are they getting so much of a bigger answer? How could I show a kid where something in here is getting counted in 
here also? So, this is trying to count all the things that are face cards but not hearts, they seem like disjoint situations. But they have to be overcounting. Or was mine wrong? Is mine, 'cause here, something that's a face card and not a heart, so that's like a king of diamonds, that isn't getting counted in here, because these are strictly face cards and hearts, so there's no way that these two face cards can be the same. The second card could be the same, so maybe, so maybe mine is under counting. So, could I get the same, what is mine taking out?

I then asked if any other diagram had come up for her at all (as she considered the alternative), and she said that it had not, but that she might be able to make sense of one.

P: So, did I take out too many things? Oh I did take out too many things. Because there are 3, but, did I? So the 12 times 13 is 156 , and if I take away 3 I get their same answer... So, are there only 3 situations in which, bad things happen? Or is it really 3 times 12 ? Gosh but I'm very convinced that like, having, like if you had the jack of hearts, that then there are 12 different ways that you could have that jack of hearts, so there are 12 scenarios in which this, like, sort of overcounted. And also for queen of hearts, so I really think mine is right. So how does theirs not take into account? So I guess I'm saying that when you have a jack of hearts and then like a 1 of hearts, or a jack of hearts and then a 2 of hearts, that these are all things that are getting overcounted. And there's going to be 12 of these, so they're not, somehow they're not saying that that is problematic. Gosh I don't, I need a... I really think my reasoning is correct, but I also am having a hard time finding a place where theirs is overcounting.

Paige then drew a diagram (Figure 35) that was different than the one she had constructed previously. This time, she drew a Venn diagram in which the sets involved were single cards (not pairs of cards). She drew circles representing the set of face cards, and the set of hearts, and their overlap, and in fact she identified each element within each set. There were thus exactly 3 cards in the intersection of the two sets - which she labeled as the jack of hearts, queen of hearts, and king of hearts. 


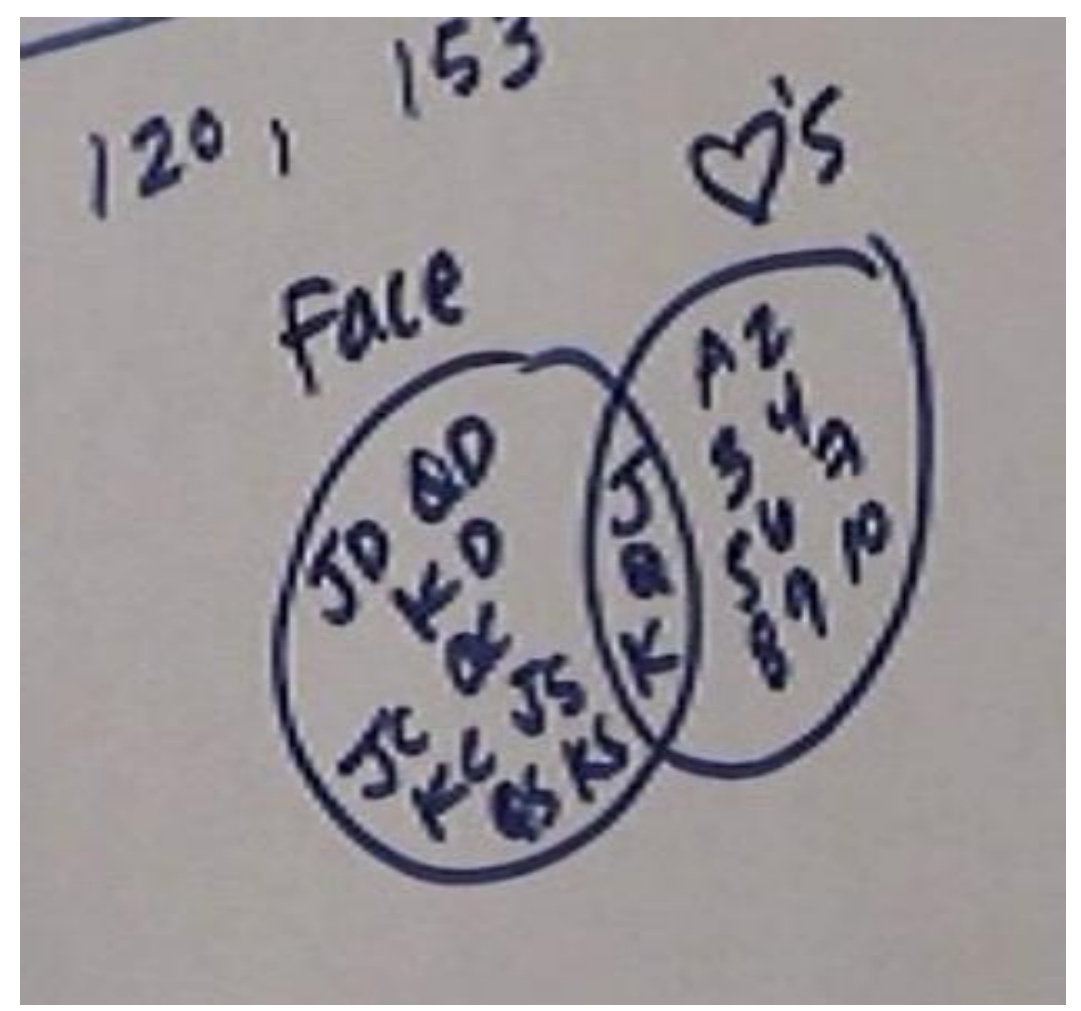

Figure 35

Paige recognized that the correct answer was only 3 different than $12 \cdot 13$, and she considered that relationship for a bit. She was, however, ultimately not able to make sense of which answer was correct - whether she should subtract 3 or $3 \cdot 12$ as she had originally done. Mathematically, while she was correct that the jack, queen, and king of hearts all have twelve scenarios associated with them, not all twelve of those situations are actually overcounted. Indeed, the only scenarios that could get overcounted are when both the first and the second card are both identical face cards and hearts. She continued to think about the problem, but ultimately she did not arrive at an explanation for the discrepancy. 
4.2.2.3.3 - Summary. This ended Paige's work on the problem, and ultimately she was unable to determine which answer was correct and why. While she drew Venn diagrams and had some set-theoretic tools at her disposal, she did not coordinate everything sufficiently in order to make sense of the fundamental issues. While $3 \cdot 12=36$ cases made sense as the number of possibilities associated with the jack, queen, or king of hearts being the first card, she did not see that not all 36 of those options actually get counted twice by $12 \cdot 13$. Some of the most noteworthy features of her work on this problem were her uses of the Venn diagrams (particularly that she used pairs of cards in one instance and single cards in another), and also her struggle to make sense of the problem in the end. I conjecture that it is not the case that Paige neglected to consider the set of outcomes at all - in fact on multiple occasions she seemed to draw upon the set of outcomes. Rather, Paige's struggle with the problem arose because she could not get a solid enough handle on what she was counting, and in particular how her counting process was subtracting too many outcomes.

4.2.2.4 - Other episodes. While I have discussed three students' work on the Cards problem in detail, I now address one other interesting feature of part of one student's work on the problem. Specifically, I chose this episode in order to provide an example of a student's explicit set-theoretic language.

4.2.2.4.1 - Casey uses explicit set-theoretic language. After some initial work on the problem, Casey got the correct answer of $3 \cdot 12+9 \cdot 13$. When asked how he arrived at this answer, he explained that he considered the number of possibilities in a set of outcomes, and that he considered two kinds of possibilities and added them together. This 
language suggests to me that he related counting processes and sets of outcomes, resulting in set-oriented thinking - that he organized the total desirable outcomes into different types of desirable outcomes, which he then added together to find the total.

E: Great, and so can you - that makes what you said. How did you know then to go to the $9 \cdot 13$ plus the $3 \cdot 12$.

C: Well I figured you could break it up into two different cases, because the - given the option of choosing a face card and then a heart, it can either be a non-heart face card and a heart or a non-heart face card and a heart, and you add those together - and so you have a certain number of possibilities in this set of outcomes, and a certain number of possibilities in this set, and you just add them together and that would be the total number.

He also talked about his visualization of the problem, and he articulated some setoriented ideas below. Note, in the excerpt below we see that he talked about sets - but the sets referred not to the set of outcomes but to the possibilities he had (heart face cards and non-heart face cards). However, his attention to the set-theoretic ideas proved useful, and his attention to the mutually disjoint subsets explained his choice of case breakdown.

E: Okay, can you say more about visualizing the problem?

C: Well, if you're going to pick a face card and a heart, then, what can I say, then there are - I figured there are only four different sets - or four different suits for the first card - so it's either one of three, or it's the last one. So in that way you can split the choice of the first card up into two, um, subsets, and they're mutually disjoint is the word I think. And then for each one of those sets observe what the options for the second card are, which is independent of what goes on in, um the card you can pick with the first set, one of three suits, has no relation to the card you can pick for the second set, because it's a different set of events that you're assuming.

Then, I asked him one more question about the sets, and he gave the following explanation that involves sets of outcomes. 
E: Okay, great now you had something about, you used some set language, you've talked about total outcomes and, um, so can you say anything about what you're, not necessarily picturing visually, but how you think about a problem like this. Were you thinking of pairs of cards and you can, you know, you're counting up this number of pairs of cards in one case and this number of pairs of cards in another case and you're adding them together. Or, you had mentioned the word outcome...

C: Yeah, well I think, um, let me see, so I think for each one of these, for each, for each subset of possibilities to use that language, I figure for each face card you have, I don't know I kind of picture it like a tree in my mind, like a tree of outcomes like this, you pick one card, and then you have 13 different choices for the second one, the second one you have thirteen, and so the math works out to be just multiply the two together.

E: Okay, so you're picturing, in forming those, the $9 * 13$ and the $3 * 12$, you're picturing trees?

C: Essentially, yeah. Yeah. Yeah 9 trees of 13 branches (inaudible).

He described a tree of outcomes, and the 9 branches of 13 represented the outcomes in which the first card was a face card but not a heart. I am particularly interested in his "subset of possibilities" language, which indicated to me that he considered outcomes. So he used set-theoretic language both to discuss the sets of choices he had (face cards that are hearts and face cards in general), but also to describe his set of outcomes.

\subsubsection{5 - Overall Summary of the Cards problem}

Students' work on the Cards problem varied. In the discussion above I highlighted the particular ways in which students' diagrams contributed to their problem solving activity. We saw in Peter's work a way in which a well-drawn diagram was particularly effective in helping him identify an error. In Paige's work, however, we saw that her Venn Diagram was not particularly helpful for her as she solved her problem. In the excerpts above we also saw further evidence for the potential effectiveness of students' uses of 
particular outcomes. For Brandon, my suggestion to write down a particular example was arguably an important step in his success on the problem, and a similar claim could be made about Paige's articulation of a particular outcome. Peter wrote out some examples when prompted, but the particular instances he chose were not illuminating for him and were not problematic examples. This concludes our discussion of the Cards problem. We now turn to students' work on the Groups of Students problem.

\subsection{3 - The Groups of Students Problem}

The Groups of Students problem asks, "In how many ways can you split a class of 20 into 4 groups of 5?" Students generally struggled on this problem, with only 4 of the 22 students initially arriving at the correct answer. In contrast to, say, the Passwords problem, on the Groups of Students problem I did not use two alternative solutions that could have been numerically equivalent. That is, the correct answer I sometimes gave as an alternative answer,

$$
\frac{\left(\begin{array}{l}
20 \\
5
\end{array}\right) \cdot\left(\begin{array}{l}
15 \\
5
\end{array}\right) \cdot\left(\begin{array}{l}
10 \\
5
\end{array}\right) \cdot\left(\begin{array}{l}
5 \\
5
\end{array}\right)}{4 !},
$$

and the incorrect answer that I gave,

$$
\left(\begin{array}{l}
20 \\
5
\end{array}\right) \cdot\left(\begin{array}{l}
15 \\
5
\end{array}\right) \cdot\left(\begin{array}{l}
10 \\
5
\end{array}\right) \cdot\left(\begin{array}{l}
5 \\
5
\end{array}\right),
$$


quite clearly differ by a factor of 4 factorial $^{35}$. As a result, the dynamic in which the students tried to consider whether or not two expressions (and the processes that underlie them) are the same did not happen here. The problem still yielded a number of opportunities for some meaningful discussion, though - because the two expressions are clearly different, students immediately tried to see which was right or wrong. In my discussion of this problem, there are two major expressions to which I will refer. For the sake of space and efficiency, in this section I will refer to the correct answer

$$
\frac{\left(\begin{array}{l}
20 \\
5
\end{array}\right) \cdot\left(\begin{array}{l}
15 \\
5
\end{array}\right) \cdot\left(\begin{array}{l}
10 \\
5
\end{array}\right) \cdot\left(\begin{array}{l}
5 \\
5
\end{array}\right)}{4 !}
$$

as "Expression R" (for "Right") and the common incorrect answer as

$$
\left(\begin{array}{l}
20 \\
5
\end{array}\right) \cdot\left(\begin{array}{l}
15 \\
5
\end{array}\right) \cdot\left(\begin{array}{l}
10 \\
5
\end{array}\right) \cdot\left(\begin{array}{l}
5 \\
5
\end{array}\right)
$$

"Expression W" (for "Wrong”).

Another important feature of this problem is that I needed to know the way in which students interpreted the problem. This is always important in counting, but it was particularly relevant for this problem. Specifically, the distinction between the correct answer and the incorrect answer I chose to present hinges upon whether or not the four groups of students are considered distinguishable. The problem as stated intends for the groups not to be distinguishable, and so Expression $\mathrm{R}$ is the correct answer; if the groups are distinguishable, then Expression $\mathrm{W}$ is actually correct. This issue of interpretation

\footnotetext{
${ }^{35}$ The rationale for the choice of the particular alternative answer is seen in the Methodology chapter.
} 
came up in my very first interview, when a student who initially gave an incorrect answer (without the division by 4 factorial) had assumed that the groups were distinguishable. When I asked about how he interpreted the problem, he immediately realized that he had taken the groups to be distinguishable and had thus overcounted. It was clear to me that students' interpretation would mean the difference between whether Expression $\mathrm{R}$ or Expression W was correct. I thus almost always asked students how they interpreted the problem (in one or two cases I simply forgot). In some instances, this question became something of a prompt, affecting their subsequent work on the problem, but it was important to me that they had the correct interpretation the problem, even if that meant, in some cases, that questioning their interpretation affected their work in some way.

All 22 students attempted the Groups of Students problem, and I revisited the problem with 17 of them. Of the initial responses, 4 got the problem right; 3 of these got Expression $\mathrm{R}$ as the answer, and the fourth correct answer was $\frac{20 !}{5 ! \cdot 5 ! \cdot 5 ! \cdot 5 ! \cdot 4 !}$. Another 12 students gave the incorrect answer of Expression B, while 6 students gave some other incorrect answer. This problem was met with the lowest success rate of any of the problems in the study; 4 of the 22 students initially got it correct.

\subsubsection{1 - Makaena's work on the Groups of Students problem}

4.2.3.1.1 - Overall description. Makaena began by writing out four circles, with five dashes in each circle, and she arrived at the initial answer given in Expression W. She explained her multiplication, and while she said she was somewhat hesitant, she talked her way through her answer and believed that she was correct. In doing so she did not 
seem to consider the set of outcomes; she indicated that she did not consider any particular instances of what she was trying to count. When we revisited the problem, I immediately showed her Expression R, and she seemed to make sense of it. To convince herself, she went to a smaller case, and she systematically listed some outcomes for a smaller case. In doing this she realized the nature of the difference between Expressions $\mathrm{R}$ and $\mathrm{W}$, and she saw the error in her initial solution. In subsequent discussion, she made comments that suggested that she considered the relationship between counting processes and sets of outcomes. Overall, she was successful at evaluating the alternative solutions, and she was able to detect the error in the incorrect answer and to utilize set-oriented thinking to do so.

\subsubsection{2 - Detailed account}

4.2.3.1.2.1 - Makaena arrives at an initial incorrect answer. Makaena initially drew four circles with 5 dashes in them, representing the four groups of five people. After some thought, she noted that 20 seemed like a big number to work with. She considered working with a smaller case first, though she was hesitant because she felt it would be hard to keep the integrity of the problem. In the excerpt below we see that she did reason through some terms, which she multiplied together; she argued that she could first choose 5 students to be in "this group" (referring to the first circle she drew), and she wrote $\left(\begin{array}{l}20 \\ 5\end{array}\right)$ under the circle. She then wrote $\left(\begin{array}{l}15 \\ 5\end{array}\right),\left(\begin{array}{l}10 \\ 5\end{array}\right)$, and $\left(\begin{array}{l}5 \\ 5\end{array}\right)$ under the remaining respective circles, thus arriving at Expression W. In the underlined portion, she noted that she was worried that she may have missed something. While this utterance could suggest 
that she considered a set of outcomes (the fact that there is some outcome that was not considered), her activity here suggests that she was primarily applying a counting process and arriving at a formulas/expression, working almost entirely independently of the set of outcomes.

M: Um, hmm. 4 groups of 5, yeah because if I just, let's say I take 20 and I pick, choose 5 of them to be put in this group... Okay, well, that at least then for the next group maybe I'd have 15 choose 5 , and then 10 choose 5 and then that would kind of, well 5 choose 5 would be 1 . There's only one way to put those people in there. So, I guess my, probably want to multiply these together. I'm just worried that's, um, that doesn't consider everything. So, (inaudible) choose 5 of them to be in this group, then I have 15 left to put in this group. And if those 5, well I guess that takes care of all the different types of combinations of any 5 people, it doesn't matter where they sit. Hmm.

I had asked her why she decided to multiply the terms together, and she explained the multiplication in the following way. She explained that for each way of choosing 5 people from the 20 , there were a number of other ways to deal with the remaining 15 people.

M: Well, let's see. There's 5, so there would be, well let's just get a number here...20 choose 5. Whoa, these are huge numbers. 15504 ways to put 5 people into just a group. Um, but once you've done that, you still have 15 people to put in different places... And so, each way you can do that would then, um, you know you pick one of these, and then there's all these other ways to do this. And then pick one, you know for each one of these there would be some different combination here.

As I continued to ask about her work on the problem, she indicated again that she was not completely convinced of her answer. She feared over or undercounting, but she did not address specifically how her answer might be incorrect. We see below that she did talk through her reasoning again, but she did not appear confident in her original answer. 
M: ... I'm a little worried that maybe I'm overcounting or undercounting something. But this seems like it would work, uh, this is just, make a group, see what's left over, make a group with that. And then, but this takes into account any 5 people being in that group. And then this is any other 5 people, so I think that, hmm.

Makaena went to calculate the numbers, but I explained that I did not need the specific numerical values. She said finally that the answer seemed correct, but she would want to work on the problem a bit more.

As mentioned above, a student's interpretation of this problem was particularly important, because a student's answer could depend very much on whether or not they interpreted the problem as I intended it. I therefore asked Makaena how she interpreted it, if she thought about breaking the class into groups that were indistinguishable (though I did not use that word), or whether the groups were different from each other. As we see, she interpreted the problem as intended. I also asked if she considered any particular examples of what she was trying to count, and she indicated that she had not. This discussion concluded her initial work on the Groups of Students problem.

\subsection{2 - Makaena investigates a smaller case, in which systematic listing proves useful.}

We then revisited the problem later in the interview, and I gave Makaena the correct Expression R to start our discussion.

M: Okay. Ah, okay this one's very similar, they divided by 4 factorial. Yeah I was a little worried about that because of these groups, you know like if they were all sitting at table A... This is table A, B, C, and D, you know what if I put these people at table $\mathrm{A}$ and these people at table $\mathrm{B}$ or something like that.

Right away, she seemed to recognize what the 4 factorial might be doing, and she suggested that she had been worried about a similar kind of issue. This shows that she 
was able to examine a particular expression and to explain a counting process that might underlie it. We see that she had basically explained the underlying issue (she expressed it as the same people at different tables representing the same division of people), but she wanted to investigate it more.

Makaena decided that she wanted to attempt a smaller case in order to be sure about the role of the 4 factorial, and she worked through a smaller case of dividing 6 people into 2 groups of 3 . She first wrote down A, B, C, D, E, F to represent the people, and she wrote two circles with 3 dashes each in them. She noted that if she applied her initial method to the smaller case, she would get $\left(\begin{array}{l}6 \\ 3\end{array}\right) \cdot\left(\begin{array}{l}3 \\ 3\end{array}\right)=20$, which she noted would not be too bad to write out. She then stated that if she applied the other expression to the situation [Expression W], she would get 10, and she decided to write some examples out to see if she would get 20 or 10 as her answer for the small case.

Makaena then wrote out divisions of 6 students into groups of 2, and she wrote out ABC DEF, then CEF ABD, and then CDF ABE as possible divisions of the students. She paused and then wrote DEF ABC, and noted that this was the same as something she had already written. This activity is noteworthy because in this smaller case, she actually wrote out particular outcomes. Her activity here suggested that she considered the set of outcomes and related it to a counting process. 
M: Um, alright A, B, C and then that forces DEF here. So that's one. ABD CEF. ABE and $C D F$. Hmm. Let's see, oh right, because the first 3 could have been DEF, and then I would have been forced to put $\mathrm{ABC}$ in this group, but that's really the same, so these [referring to $\mathrm{ABC} \mathrm{DEF}$ and $\mathrm{DEF} \mathrm{ABC}$ ] are really the same. Um, oh. Oh, okay so you divide by 2 because, so how would that happen up here? Maybe you chose ABCDEF here and then in the next slot they could have been chosen this way, that's no different than that. Okay, yeah, I think that this double counts because if I just choose 3 people, it could have been A, B, and C, and then that forces DE and $\mathrm{F}$ in the second group. But, let's say the first three people were $\mathrm{DEF}$, that forces $\mathrm{ABC}$ in the second group, and that's exactly the same, just, it doesn't matter, there's, there's, ABC are in a group and DEF are in a group. So this double counts this, and I think, then, dividing by 4 makes sense.

As we see, she explained the double counting by referring to her initial solution; she identified two outcomes being "exactly the same," and her language underlined above suggested a coordination of a counting process with the outcomes it generated. At the end of the excerpt she returned to the original 20-person problem and considered whether division by 4 (factorial) made sense. I then asked her to expound upon her work a bit more and explain what she had done, and the following exchange took place.

M: Sure. Okay. So I thought about, well, let's look at a smaller case, say I have 6 people, so I listed them out, ABCDE and F, drew my little picture and then went with the method that I chose, um, when I first did the problem, and so, uh, 6 choose 3 and that leaves me with 3 choose 3 ,

E: Okay.

M: Uh, and I got 20, and then I thought, okay I want to compare that to what this method does [the method in Expression R], which is then to divide out by the number of ways to arrange those groups of people. So here it was 4 factorial, here it would be 2 factorial, which is 2 , so 20 versus 10 . So I started making these theoretical groups, um, and as I was listing out thinking, oh I don't want to list them all out, what would happen later on in the alphabet? You know it wouldn't just be A, B, and C. Later on I might get a DEF in the first group, but then that forces $\mathrm{ABC}$ in the second group. Um, and then I realized oh, that's the same thing here, and I, using this method it counts them each separately as one, uh, this is one 
way, this is another way, but they aren't really. So, um, this [referring to ABC $\mathrm{DEF}]$ would be double counted. And then let's say, so then here, it's not just halving it, like I don't think it would make sense just to divide this by 2 ,

In the excerpt above (particularly in the underlined portions), we see that much of her discussion seemed to indicate the coordination of the relationship between counting processes and sets of outcomes. Also, her mention of "theoretical groups" is interesting. This language is reminiscent of Marcus' "hypotheticals" that he described in the Passwords problem.

Makaena went on to articulate again why the division by 4 factorial made sense. She briefly mentioned a case in which 9 students might be broken into 3 groups, and she, through an example, explained why division by 3 factorial made sense in that instance. She concluded then that division by 4 factorial in the original problem made sense.

M: Um, even though this is what, you know, we're dividing it by 2 , because let's say you had in this larger case, or a larger case, ABC and then you had DEF and $\mathrm{XYZ}$, well, that would be the same as, I could reorder any of these so, uh, I'll call this group 1, and 2, and 3, so then I could, I could have chosen DEF first and then $\mathrm{ABC}$ and then $\mathrm{XY}$, okay, so, there's 6 ways to arrange this...3 factorial is 6 , so, um, dividing it by 4 factorial makes sense.

I then asked her what she felt the listing out of groups did for her.

E: And so was it, kind of being able to see exactly how that DEF ABC might show up a second time that made you see what happened, or,

M: Yeah I didn't really realize, yeah I guess it's hard to see if you're double counting if you don't actually write out some cases. And, um, I also don't feel as comfortable. I kind of want to know what these things look like before I start counting them, and...

E: Okay, so you had kind of written those circles with the slots as placeholders, but you wanted some particular examples in there?

M: Right, right. 
She indicated that her act of listing contributed to her ability to detect an overcount. She saw the benefit afforded her by the actual physical listing of examples. She noted, too, that she had wanted a sense of what the objects (the groupings of students) looked like. It thus seemed that at that point in her work, she recognized the value of considering the set of outcomes.

\subsection{3 - More evidence of the relationship between counting processes and sets of} outcomes arises as Makaena reflects upon the problem. I then asked Makaena how she might explain to someone else why the 4 factorial is important, if someone came to her with Expression W. She talked through an example and highlighted what would happen as people were chosen to be in different groups - that the same 5 people could have been chosen first or second, but that would not necessarily mean a different division of the 20 people.

E: So what if someone came to you and gave you, you know just the numerator there [Expression W]. How would you explain what, or, I mean same kind of deal, or...

M: Yeah I guess I would draw a picture. And say, okay well let's, um, you know imagine picking 5 people and putting them in this group, and then I'd actually list out either some names or some letters or something like that. And, um, okay, let's pick 5 out of the rest of the people, put those in here [one of the circles she drew]. And then have them imagine, um, saying okay, since in the beginning we could have picked, um, 5 from the whole group, what if it had been the 5 that were picked here in the second group, what if those [5 other people] were picked first? Right and then that second group was now it could have been any of those people from the first group, and let's say that was there. Is that a different set of four groups, or is it the same? And that kind of thing. So I think examples would be the most persuasive in that case. 
There was one statement in particular in the excerpt above that suggested to me that she considered a set of outcomes. She said, "Is that a different set of four groups, or is it the same?" That indicated to me that she considered the objects she was counting to be "sets of four groups" (or one particular division of 20 people into four groups). She did not want to consider two sets of four groups to be different if only the arrangement of the groups was different. This different and same language came up many times for students (not just on this problem), and in fact it became a primary way for students to articulate that overcounting had occurred. When students revisited their work, there were no less than 48 instances in which they talked about outcomes being the same or different. On the Groups of Students problem alone, there were 12 such instances.

I then asked her how she thought about the correct answer, Expression R. I was curious if she thought that the numerator imposed some order, and the division by 4 factorial corrected that order, or if she thought about the numerator as generating some set of outcomes and the four factorial was eliminating repeated elements.

M: I think my first instinct would say that order was accidentally imposed. Um, because I sort of picked these people first, and then I picked these people second... Mmm, but, I probably could have picked these people first [she pointed to the first cell of five in her original work] and these people second [she pointed to the second cell of 5]. And it's still the same two groups.

In response to my question, she pointed to the first and second cells of 5 in her original problem, and she noted that because she picked groups of 5 people first and then second, she actually considered that the order in which the groups of 5 were chosen mattered. She could have picked two groups in the other order, and it would still be "the same two 
groups." Makaena thus recognized that she needed to consider carefully whether her process resulted in counting two outcomes as different that were actually the same. Because the two outcomes she described above were the same, she needed to make sure she counted them as the same. Her activity here again highlights the relationship between counting processes and sets of outcomes.

4.2.3.1.3 - Summary. Makaena experienced great success on this problem. While her initial answer was incorrect, she was able to make sense of the correct answer and to justify why it was correct (and why hers was incorrect). In particular, she utilized systematic listing within a smaller case in order to recognize her error. Her work was marked by a coordination of counting processes and outcomes that the process generated, and she realized that her solution was problematic because certain ways in which the students were divided were counted as different, but were actually the same. This episode is an example of how the coordination of the relationship between counting processes and sets of outcomes was beneficial in helping students make sense of a counting situation, and particularly how language of "the same" and "different" facilitated communication of an overcounting situation.

\subsubsection{2 - Zach's work on the Groups of Students problem}

4.2.3.2.1 - Overall description. Zach began the problem by writing 20 slots, and he modeled the problem as arranging four sets of five letters each in those 20 slots. His overall strategy was to first knowingly overcount (he began with 20 factorial) and then to divide in order to correct the overcounting. He went on to address some of the 
overcounting, and he felt that he was perhaps done, but by briefly considering a smaller example he realized that he needed to do more on the problem. When he saw the alternative expressions, he made sense of them, and we spent time discussing how he could be sure he was "done" correcting for the overcount. Throughout his work on the problem, his language suggested an ability to consider the set of outcomes, and it seemed as though he could easily and naturally relate his counting processes with sets of outcomes.

\subsubsection{2 - Detailed account}

4.2.3.2.2.1 - Zach models the problem as an arrangement of letters. Zach first drew 20 slots, which he called his "go to picture" (Figure 36 below).

Z: Oy. Okay, my first reaction to this, um, is to go to my go to picture, I suppose...Um, let's see, and I'm going to let each of these blanks represent a distinct student in my class of 20 , um, so that student 1 is that blank, um, and to decide what group student one is going to go in, I'm going to, I'm going to, I think I'll use letters. Um, I need 4 groups of 5, so I'd like to put four different letters in each of these blanks, um, so there will be...4 different letters of which, of each there will be 5, so, I'll have, um, 5 a's, 5 b's, 5 c's, and 5 d's, for example, um, sprinkled into this. Um, so I guess it's become a question now of how many ways are there, um, distinguishable ways are there to arrange those 20 letters in these blanks. 


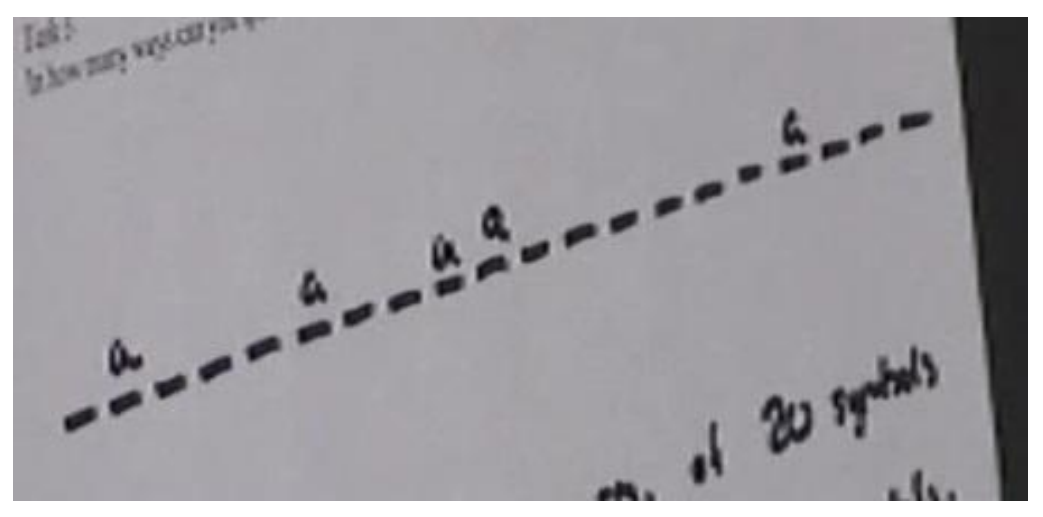

Figure 36

As Zach began, he noted that he considered the blanks to be numbered, representing Students 1 through 20, respectively, and he thought about sprinkling five a's, b's, c's, and d's sprinkled into his picture. He thus essentially turned the problem into counting the number of ways he could arrange four sets of five letters each in the blanks. As he continued to reason, he seemed to consider the situation and the objects he was counting.

Z: Um, so, um, well, if, if I take those 20 letters and were to give them all slightly different colors, so uh, there really were 20 different symbols in some sense, uh, the letter a that is green is, in some sense, different than the letter a that is blue or something like that, I would have 20 different symbols, um, there would definitely be 20 choices for which of those symbols I placed here, 19 here, and 18 here, um, so that's, that's the first thing I would do, but then, it's not really, I don't really care that those, those uh 5 a's for example, that's just telling me that that group of 5 students is all in the same group, um, and it doesn't really matter whether Ted got a green a and Sue got a blue a, um, they're just in group a. So I think I need to now divide out, um, I need to divide out the fact that I've way overcounted this thing. Like, one particular arrangement I think I would have been overcounted by 5 factorial just in the a's alone.

With his 20 dashes in front of him he could actually think about features of the outcomes, as opposed to simply manipulating numbers. As he tried to count the arrangements of 5 
a's, b's, c's and d's in 20 spots, he noted that if each letter was a distinct color, he would just have to arrange 20 different objects, yielding 20 factorial. He did not want to consider the a's as being different colors from each other, however, and he adjusted accordingly by dividing by 5 factorial. The underlined portion shows that he realized overcounting occurred, and his reference to "one particular arrangement I think would have been overcounted" suggests a that he considered the relationship between his counting process and the set of outcomes.

As he continued to work he wrote out 5 a's in 5 of the dashes, and called them the red a, blue a, green a, yellow a, and purple a, respectively. He then pointed to the locations of the a's again and noted that the different colored a's could be in different positions.

$\mathrm{Z}$ : Um, this is how many ways I can arrange my symbols while they have color, and this is me saying, eh that color thing was just a device to get me started, because those 5 a's that are all in there somehow, someway, I can scramble those 5 things up. For example, maybe the a's got placed here, here, here, here and here. Um, then, ignoring everything else in this diagram, I know that if that was the red a and the blue a and the green a, yellow a and purple a or something like that, um, that I could scramble, I could choose any one of those different colored a's to be in that spot, any one of the four remaining a's to be in this spot, and 3,2 and 1, and that would be totally indistinguishable, they're still in "group a," um. And that's just worrying about, um, taking out all the overcounts of that one particular group of 5 students that are group a.

This excerpt is noteworthy for two reasons. First, he considered an aspect of a particular outcome in order to reason about his work. That is, he considered a possible placement of the a's, and he used that as a concrete example that could aid in his decision. Second, his discussion suggests that he was considering the outcomes - that he could think about (and argue about) particular outcomes that his counting process was generating. This provides 
an excellent example of the relationship between counting processes and sets of outcomes. After the discussion in this excerpt, he had arrived at the expression $\frac{20 !}{5 ! \cdot 5 ! \cdot 5 ! \cdot 5 !}$ as a possible answer to the problem (this expression is numerically equivalent to Expression W).

4.2.3.2.2.2 - Zach utilizes a smaller example as he accounts for more overcounting. Zach then saw that there may have been a mistake in his work described above. In particular, he realized that his answer might be overcounting, and this came up because he, quickly and in his mind, considered a smaller problem in which 2 people were broken up into two groups. He explained this in the excerpt below, and I asked about what made him pay attention to that smaller example.

Z: Um, hmm. I'm suddenly less happy with this than I was just a second ago. Well, it's just, this isn't good, because I think I'm going to be, hmm, I think I need an entirely new strategy. And the reason I'm thinking I need a new strategy, um, is because I was thinking of a much smaller problem, and my answer to that problem doesn't make any sense (laughs). Um, I think if you had two students, and you wanted to split them up into groups of one, um, I would have just said there was 2 ways to do that, following the same format, and I don't believe that that's true. There's exactly one way to separate the two students into two groups.

E: Okay so you, as you were talking through that, you went through a smaller example in your head, and just, just as kind of a reasonability check?

Z: Yeah, I was just, each of these felt reasonable, but I was starting to worry about the fact that while I've got these, that group of students kind of all in a, um, not only could they have all a's sitting here, but that, if I'd have put all the different colored b's on here, I think I'm counting that as an entirely different arrangement... Um. So, my heart is wanting to believe that I just put a 4 factorial on the bottom and I would be done with it, but, um, because that could have been, the a's and b's and c's and d's could have been scrambled in any which way. 
In the first underlined portion, he realized that those 5 a's that had been in his diagram actually could have been b's, which could have resulted in the same assignment of groups (though he was counting them as different). In the second underlined portion, he suggested that perhaps another division by 4 factorial would fix this issue. His discussion and language here indicated sensitivity to considering outcomes.

I then asked him what made him see his error, and his language suggested an ability to coordinate his counting process with certain outcomes.

E: And did, was, did looking at the diagram make you see that, or what made you worry about that just now?

Z: I think it was, um, my entire strategy is to take away all the over-counting that I'm doing, and so I was, I was kind of focused on, well have I really done that, have I taken away all of the duplicate countings, um, that aren't really distinguishable, and it was as I was getting ready to talk about the students labeled b that I realized I might just as well have had these labeled $b$ and the other ones as labeled $a$, and I'm counting that as exactly, as two different things here.... Um, which didn't feel good. So, my heart is to say that a's, b's, c's and d's could all also, I could replace the a's with b's and c's, etc, and all those 4 factorial ways of arranging those letters I think need to belong here, um, as well.

He also was articulate as he described what his procedure was doing globally - namely that he was initially counting something he knew to be too big (the 20 factorial) and then taking care of the overcounting. To fix the issue of the ordered groups, he decided to divide by 4 factorial, thus arriving at a solution of $\frac{20 !}{5 ! \cdot 5 ! \cdot 5 ! \cdot 5 ! \cdot 4 !}$. Zach used his language about counting something as two different things as a way to talk about overcounting, as Makaena had. 
Zach wanted to revisit the smaller case to see if that division would make sense, and he talked through the smaller example that had initially caused him to re-evaluate his work. In the underlined section even though he convinced himself of the smaller case, the sheer magnitude of the original problem made him less than confident in his final answer.

Z: Um, however, I want to see if that actually, um, is consistent with the smaller example that I'm thinking of, just to see if this is even plausible at all. It makes me want to go back and think about, maybe an entirely different approach to this problem. But, if I had a class of two, um, something I know I can deal with reasonably quickly, um, into two groups of one, just re-writing your question in something easier.

E: Yeah.

Z: Um, then I, I know that there's one way to do that, you just send one kid to one side of the room, and one to the other, that's satisfied. Um, if I go with my lettering scheme, I've got two blanks, and, um, I'm going to put an $a$ and a $b$ in there somewhere, and a could be in, there's going to be exactly one a somehow in there, um let's see, so I think there's 2 factorial ways of placing my symbols, if I'm going with, trying to make this, uh, fit, of which there's exactly 1 factorial way of shuffling that one a up with itself. Um, but then I'm going to divide by the fact that I've got two different colors in play and it wouldn't have really mattered which way I did it. So, that feels to me like it's at least more correct than what I first wrote down. Um, however, the numbers are large enough here that, um, my current level of comfort with this answer isn't very high. Um, it feels plausible to $\underline{\text { me, and it feels like I've, um, gotten rid of my overcounting, but at the same time I }}$ thought I had done so the first time I wrote something down, and it was no, so, I'm a little bit dubious about my answer at this point.

Finally, I asked him about his interpretation of the problem, and he indicated that had interpreted the problem as I had intended.

\subsection{3 - Zach examines alternative answers, but he does not see them as the same as}

his answers. When we revisited the problem, I gave Zach both alternative expressions and asked him to make sense of them. He recognized what each expression represented, 
realizing that Expression $\mathrm{R}$ was trying to take care of overcounting in a way that Expression W was not. Again this suggests that he could examine an expression and relate it to a counting process. Below we see he revealed a particular way to think of the overcounting - "team names" - that would come up in his subsequent work on the problem. He said that he did not care if the groups of students were named anything different, they were still just different teams.

Z: Okay. This student elected to fill, of the 20 blanks elected to put 5 a's down, that there's 20 choose 5 ways of doing that, um, and then said well, now I've got 15 remaining blanks, so I should put 5 b's down in them. 10 remaining blanks, I will put 5 c's down, and then of the 5 remaining blanks I'll put 5 d's down, and left it at that. Um, which certainly will fill in this entire string with letters. Um, but it's definitely going to overcount things, but before I talk about how much that's overcounting I, this student did exactly the same thing, but then said, oh, we'd better account for, for a little bit of overcounting, um, namely the fact that I don't really care if this is group, Team A and the other one is Team B, I, they're just two different teams, so, um, that's, that's trying to account for the a's and b's things. Um, now I wasn't happy with my answer to this, so, I don't know, I don't know at this point in time, I feel better about what I've said than 20 choose 5, 15 choose 5 , 10 choose 5 , and 5 choose 5 , um,

I asked him about whether his answer matched Expression R, and he initially said that it did not, because he thought the numerical answers were different. He then worked through some of the computations and decided that they were equivalent expressions. Zach said that the fact that his answer was numerically equivalent to one of the alternative answers made him somewhat more confident in his own work.

\subsection{4 - Zach expresses concern about when his correction of overcounting is}

complete. Zach explained his work again, and we see below that he made an insightful 
observation, specifically that his process made him feel less confident because he was intentionally overcounting initially, and then later taking care of the overcounting.

Z: Okay, uh, my approach was to, I knew for a fact I was way overcounting things when I put a 20 factorial down. Um, and then I was, so I was going from the most ridiculous overcount you could ever possibly have in this problem, and then trying to strip away the things that, um, shouldn't have been counted more than once. Um, and the problem with that approach is that I had a hard time really believing that I had accomplished my goal. I knew that these things were, were getting me closer to my goal, I was, um, I was getting rid of duplicate counting each step of the way, but there's, there was really no flag saying, okay you've officially now accomplished your goal of removing all the overcounting. Um, which, I mean it didn't really bother me on some of the other problems, but because I know I had written down something untrue here it was really hard to believe that just tacking on a 4 factorial down there really did mean I was finished.

In the excerpt above Zach noted (and this came up multiple times in his discussion of this problem) that he never felt secure in when his "accounting for the overcounting" process might actually be complete. He associated his initial process (represented by the expression $\frac{20 !}{5 ! \cdot 5 ! \cdot 5 ! \cdot 5 !}$ ) as causing an overcount, or of generating a set of outcomes that was too big and that had repeated elements in it. Then, he was able to examine his outcomes and decide that there were indeed some repeats, so he had to account for an overcount by dividing by 4 factorial. His statements above were insightful and suggest that he considered the set of outcomes.

I also asked if, in addition to comparing his expression with Expression $\mathrm{R}$ numerically, he could talk about what each of the expressions represented, as far as a counting process was concerned; I will discuss our exchange below. 
Z: I just kind of lost some confidence in that, in the answer. Um, this, this approach is logically sound, you can put a bunch of a's down and then put a bunch of b's down and c's down and d's down, and then get rid of the fact that a's, b's, c's, d's don't really matter. Um, they were just temporary labels for the team name, I guess...Um, but even in this case I don't, because I think I've kind of gained some lack of confidence here, um, I would ask that student how they know for a fact that we actually are done, that that doesn't overcount anything. Um, and, I don't know that there's a good answer to that right now, off the top of my head other than, um, smaller cases we can actually wrestle with and believe, believe that our, our construction is actually agreeing with what we know actually has to happen. Up in the stratosphere of 20 students, this number's so huge that it really would be hard to compare it to something.

E: Okay cool, so there's some question for both answers, if you're really sure if you're done, and maybe particularly for yours because you began knowing that you were overcounting and that you were going to correct stuff. So it's like, when am I done correcting things?

Z: Correct. Uh, exactly, I, uh, yeah if there was, if there was some, this, I don't even know if it's going to mean anything, but starting at way overcounting and whittling my way down, if there had been some other plan of attack that would have started with for sure undercounting and whittling - or, patching it up, if I could have found a way that those two would meet maybe I would feel better.

It is interesting that Zach did not see Expression $\mathrm{R}$ as choosing students to be in groups or at least he did not indicate that that was his thought process. Instead he thought of it as putting a's, then b's, then c's then d's down into the 20 slots. This is maybe a subtle difference, but it is markedly different than what most of the other students did. Almost all of the other interviewees considered having a group of students, and choosing successive sets of 5 of them to be in the respective groups. Also, in the underlined excerpt, we see that Zach raised the issue again of how to know when one was "done" accounting for the overcount. He also noted that the large numbers in the original problem make it difficult to get a sense of numerical verification. 
In order to get a better sense of his thinking, I asked him to explain how he would clarify to someone why division by 4 factorial is necessary. He went back to the "team name" explanation, as seen below. Also noteworthy is that he referred to a particular example (putting, say, Johnny, Sue, and Elizabeth on Team A), and this seemed to help him explain his thinking. The underlined section below highlights his coordination of the process and the outcomes - he attributed some outcomes to a process, noting that the processes reflected in Expression W count two outcomes that are the same as different.

E: No, so how would you, why the, again why the 4 factorial, how would you articulate to somebody that they need that four factorial?

$\mathrm{Z}$ : Um, I think the team name thing is a pretty plausible one. Um, this, this plopping down all these a's, b's, c's, and d's definitely successfully, um, breaks the class up into four groups of five. And the way I have done so, I would say okay, um, Johnny, Sue, Elizabeth, etc, you're Team A, um, and then this one would be Team $\mathrm{B}$, and this one will be Team $\mathrm{C}$, um, but the fact of the matter is, the same exact groups could have occurred with different team names, you could have, Team A is, um, if I said let's do a completely new arrangement, I'm going to do it again, scribble, scribble, scribble. Okay, Johnny, Sue, Elizabeth, whatever, you're now Team B, and the Team B people, you're now Team C. Well they're not going to, they're going to say, this is not different, this is not new at all. You're right, I just have given you new team names, and it's not a new scenario, so I, I definitely, this [Expression W] counts those [arrangements of the teams] as different. We know that they aren't, therefore we do need to kind of adjust for the fact that that team name business is, is just, um, it's not counting anything different.

E: Okay.

Z: It's counting something 24 times over.

And again, as we wrapped up our discussion on the problem, he articulated the issue that, on this particular problem, because he had begun by knowingly overcounting, he did not feel confident about when he could stop "whittling" away the overcount. Language 
such as "I'm taking something too big" and "trying to chop chunks off" suggests to me that he considered cutting down on the set of outcomes in some way ${ }^{36}$.

E: And do you find that's an issue with counting problems? I mean why, well like I felt like on some of these other ones you felt like okay I'm done, I feel good about this. Um, what's different about those than the instance here where you feel maybe less sure of it?

Z: This one just felt more like my, my approach, um, it was abundantly clear that I was taking something too big and whittling it... And, I probably did embedded in those other ones something similar at some point, but it felt very crystal clear here that I'm taking something too big and trying to chop chunks off. And the, I don't have very many tools for knowing when I should stop whittling, um, other than I believe I should stop whittling, I believe I've got them all done.

Finally, I asked him about how he thinks about overcounting and whittling away, and his language here, again, suggested that he thought about coordinating a counting process with some set of outcomes. The excerpt below is particularly noteworthy because it emphasizes a coordination of sets and processes.

\footnotetext{
${ }^{36}$ Cutting down on the set of outcomes and cutting down on the numerical answer amount to the same thing, but there is a difference between just reducing a number with no sense of what is being reduced, and actually cutting down on repeated outcomes.
} 
Z: But, um, I probably justify what I mean by dividing out the order by actually looking at a small case, maybe um, we want to count how many ways there are to grab two students, or three students out of a group of 10 or something like that, and I would probably justify that with, well of course there's 10 choices for my first student, etc, 9, 8, um, but, with a small enough example I could even have those students have names or whatever, I could then say, oh my goodness, if we made a big list of this, here's the 720 , um, groups like that that we could possibly have, and it, and I highlighted all the ones with the exact same students in them ahead of time, because we didn't want to spend the four hours it would have taken in class to do this problem, um, which was a relatively small problem, um, and look at how many times we're counting this one exact same group of students as different, that's just silly. Um, how many ways would there have been if we didn't want to be tedious like I was, um, how many, how many different times could we have counted Susan, Beth, and Johnny as, as being a distinct group, well, um, that's back to just the permutation stuff that would have had to proceeded combinations.

E: Okay.

Z: So we could believe that there was, um, 3 factorial ways of, of, of scrambling those three students...And then say, oh, okay, then here's our all purpose plan, um, here's what we mean dividing out the order, we just mean, we don't want this, this to possibly, or we want to count this as all exactly the same scenario.

We particularly see Zach's coordination of sets and processes in the following way. He explained how he might describe the issue of overcounting that happened in something like Expression W. He noted that if he looked at a smaller example, he might actually be able to (beforehand) write out all of the outcomes that a process generates, and then show how many of them are actually the same. He said that doing so could show that "counting this one exact same group of students as different" is "just silly." It seems as though Zach's coordination of a process with a set of outcomes was useful for him in his work.

4.2.3.2.3 - Summary. On the whole, Zach's work on the Groups of Students problem indicated that he had an ability to coordinate the counting processes he employed with the 
outcomes the processes generated. He approached the problem differently than any of the other students, turning it into a matter of arranging four different types of identical letters. He was aware of the fact that his strategy involved first (intentionally) overcounting and then "whittling" the answer down and getting rid of the overcount. This strategy made him suspicious of when he was done, so even when he arrived at the correct answer, he felt unsure of his work. His language and activity suggested set-oriented thinking, in the sense that he did not manipulate numbers and formulas in isolation of also reasoning about the objects and the set of objects he was trying to count. In terms of the model, Zach seemed to have a handle on the relationship between formulas/expressions and counting processes, and he also showed much flexibility in being able to relate counting processes to sets of outcomes. I contend that this flexibility helped him in his success on the problem.

It is also worth noting that Zach's modeling of the problem contributed to his articulation of a particular outcome. Generally, students had a difficult time articulating what they were trying to count in this problem (explaining that an outcome is a way of dividing up 20 kids into 4 groups is much more cumbersome than saying an outcome is a password). Zach, however, encoded the problem as a way of arranging identical letters, and for him, different outcomes might have been different ways of arranging and placing a's, b's, c's, and d's. I conjecture that his particular model facilitated his coordination of sets and processes, and it ultimately contributed to his success on the problem.

\subsubsection{3 - Nick's work on the Groups of Students problem}


4.2.3.3.1 - Overall description. Nick initially tried to apply the "stars and bars" technique to the problem, but then changed and arrived at Expression $\mathrm{W}$ as his initial answer; in doing this, he related a counting process with a formula/expression, but he did not draw upon the set of outcomes. He had interpreted the problem as it was intended. When we revisited the problem, I gave him Expression R, and he was able to articulate that in the correct answer, it did not matter in which order the groups were chosen. However, for some time he wavered about which expression actually gave him the desired answer, and he was ultimately not able to realize that the division by 4 factorial in Expression $\mathrm{R}$ was a necessary part of the correct answer. Thus, while he made some insightful comments about what he wanted to count (and even how overcounting might arise), and while he seemed to be able to reason about what he wanted, he did not, in the end, make sense of which answer was correct and why.

\subsubsection{2 - Detailed account}

4.2.3.3.2.1 - Nick attempts to use the "stars and bars" technique. Nick began by trying to make the problem fit into a selection with repetition model, often called the "stars and bars" technique. One other student attempted to apply this method to the problem (and two others applied the model to other problems), but it is not an appropriate model; Nick was eventually able to explain why that approach did not work.

$\mathrm{N}$ : Well first I thought it'd be, since you need to select who goes where, I did, having taken the discrete, um, learning about this method, they call it the bars and stars or whatever, the hot dog problem or whatever, Um, you have 4 groups, you can place 5 people in each one, but that, if I did that with 20 , you'd have, you can only have 5 in each group...And so I wasn't, I didn't think that would work out, 
and so I was like, well, maybe if I go with the students, because that doesn't distinguish the students apart, so if I then I went and I did the students, and I thought, well maybe, um, then I just assign each student a group, one of four groups, but that still didn't seem like it would work, because I don't think that I could set just one in - under each student, so possibly a student could be assigned more than one group.

Nick's activity here of applying the stars and bars technique is a prime example of a student considering a counting process and arriving at a formula independently of considering a set of outcomes. This exemplifies a phenomenon that can be described by the model, in which a student emphasizes the top relationship but does not consider the set of outcomes (Figure 28 above).

Nick then went to another approach, which yielded Expression W. As he spoke, he wrote Expression W, and above each of the binomial coefficients in Expression $\mathrm{W}$ he wrote "Gp 1, Gp 2, Gp 3, Gp 4,” respectively, so that each term in the product had a label above it.

$\mathrm{N}$ : So once I thought that that wouldn't work I thought of this way, and this is the only way I've seen that might seem to make sense to me, but I'm not quite sure I'm not $100 \%$ on it, 'cause, um. I did that was 20 students, so you have 20 choose 5 ways of selecting 5 of the students for the first group, then you have 15 students left, so 15 choose 5 ways of getting another one, and so on, so then you have 10 choose 5 for the 3 rd one, and then 5 choose 5 for the last one.

As he explained his solution, Nick did not mention the group names he had written above each term in the product, and so I asked him how he interpreted the problem, especially in light of his labeling of the groups. I suspected that because he had written labels for the groups, he might have been interpreting the problem as involving distinguishable groups. When I initially asked about his interpretation, it was not clear 
how he was interpreting the problem; he wanted to split them up into four groupings of 5, but he said nothing about whether the groupings were distinguishable. I was not satisfied that I knew how he was interpreting the problem, so I asked him again how he was thinking of the groups. In the following exchange, we see that he did indeed intend the problem just to be splitting up the groups, where the groups were not distinguishable from each other in any way.

E: Okay so, I'm going to kind of say two different scenarios, and I'm curious which you think that answers. So, what if you wanted to split a group of 20 into groups of 5, and send, you know, one group to music class, another group to lunch, another group to recess, and another group, say, to the library or something. Is that a different problem than splitting the class into four groups and just kind of having them sit in 4 groups and not send them anywhere?

$\mathrm{N}$ : So if you, put them in groups and send them to various spots rather than just splitting them into the groups. Yeah that's a different problem altogether, unless you pre-assigned which group was going where. Because you'd have the number of ways to place the students into the groups, and then you'd have an additional number of ways that you can get the group, which group goes to which area, rather than just splitting up the groups.

E: Okay, so, how. Okay so the fir - those are two different problems.

N: Yeah.

E: Okay so which - which do you feel like this one is asking?

$\mathrm{N}$ : That second one, where you're just splitting them into the groups and then that's it.

I also asked Nick if he pictured a particular group of students, and he said no. Also, his response below is noteworthy because it showed that he tended not to picture things. This was in line with several other students' work throughout all of the interviews (on this problem and on others), who indicated that they were abstract thinkers and tended not to go to pictures or concrete examples in their work. 
E: Okay, um, were you ever picturing, you know, groups of students?

$\mathrm{N}$ : No, not really, I'm not really a picture type of person really when I'm solving these problems too much.

4.2.3.3.2.2 - Nick discusses his labeling of the Groups. Then, because I was interested in what effect his labeling of the binomial coefficients as Groups 1 through 4 might have on his counting, I asked for more clarification of his labeling of the groups. For Nick, the labeling of the groups was organizational and helped him to keep track of the process. His statement, "keeping an order for me in my head," is curious, because it suggests that, perhaps, he saw the groups as somehow being ordered. I did not ask him more at the time, but I wonder if that indicates that he somehow viewed order mattering in the problem. He did not make the connection here (nor would he subsequently) about how the ordering of his groups would generate an overcount.

E: And then, the Group 1, Group 2, Group 3, Group 4, um, did that help you in that counting process, or what - why did you distinguish between Group 1, Group 2, Group 3, Group 4?

$\mathrm{N}$ : Um, all it really did was to, basically "I have 20 choose 5, so that gives me a group" is basically what I was thinking of, so I need to make sure I subtract those kids off for the next possible group...So it was basically just helping me keep an order on where I was at in the process, so, it was more keeping an order for me in my head.

When we revisited the problem, he explained his original answer again, and he described how he arrived at Expression W. I include his description below to highlight the sequential language - particularly the uses of "then" and the mention of "the first group" and "the last group." It seems that he was utilizing some sequential order in the ways in which he chose the groups, even if he did not intend this or was aware of it. 
$\mathrm{N}$ : Um, basically I, I kept them all as one class, so we have 20 people. In order to get into the first group we have 20 choose 5 different ways of getting 20 people into 5 - into a group of 5 . Then once you take a 5 out for one group, you have 15 of the students left, so how many ways can we choose - of those 15 that we haven't selected at another group, we get 5, and so on for each of the four groups, so 20 choose 5 , we have the 5 from that group, so 15 choose 5 , then 10 , then 5 choose 5 for the last group.

I then gave Nick Expression R, the correct answer that included division by 4 factorial and asked him to examine it. He discussed it, and as he did this he mentioned the groups by name.

E: Um, so how about this solution there [Expression $\mathrm{R}]$ ?

$\mathrm{N}$ : That's the exact way that I did it [referring to the numerator of Expression R] they're stating that, um, these groups were formed, and that these groups were formed in some order that they were required to be in, in which, um, they're saying that the permutation doesn't matter, that it's completely random on how they were placed into the groups, and so that's why they divided by the 4 factorial. Because the 20, the, like for mine group 1 could have been here, group 2 could have been there, and so basically it's the exact same answer as mine, except for mine the permutation matters because of where each one is located, which one's ordered... Where this one [Expression R] isn't. This one [Expression R] you can get them in any order.

In the above excerpt, when Nick said "like for mine Group 1 could have been here, Group 2 could have been there," he went back to his original drawing and drew arrows indicating that Group 1 could have been where Group 2 is, and Group 2 could have been where Group 3 is. The first phrase underlined in the excerpt above is confusing to me; I am not sure what he meant by it, whether he meant that the groups were formed in some order and that they had to be formed in that order, or that he was saying something else. He noted that the new answer, Expression R, was similar to his, except his took 
permutation into account. Mostly what we see in the excerpt above is that while he had some sense of what was going on (that the division by four factorial might be related to taking care of an ordering of the groups somehow), he was not clear about whether Expression R or Expression W actually accounted for the groups being indistinguishable. This would come up throughout the rest of the interview - a sense that he has an insight about what he wanted (to count divisions of 20 people into indistinguishable groups), but that he was not sure which Expression actually gave him that.

4.2.3.3.2.3 - Nick wavers between which Expression he thinks is correct. Following the excerpt above, it seemed as though he was confused about which expression allowed for distinct orderings of the groups. As he continued, his confusion seemed to deepen, as he switched several times between which answer he thought was right. In his statement below, at first it seemed he properly identified the correct answer, and he justified his decision with the right reasons (the first underlined section). However, he then changed his mind.

E: Okay, and as far as the wording of the problem goes, as you understand it, which solution do you think better answers that question of splitting the kids up into groups?

$\mathrm{N}$ : Mmm. In how many ways can you split a class of 20 into 4 groups of 5? So you get 1 group, this one [Expression R] is probably the correct one, now that I'm thinking of it. Because in mine [Expression W], order matters, where this one [Expression R] you can get, the group that you formed first could have been the group that was formed last, I think. No, because that would, that, no. I think that's still right. Because when you do this choosing, when you do this already takes into account that you're not permutating (sic). This one [Expression R] you're doing it twice. So this [Expression R] is probably undercounting I think. 
Because I did not push further about his exact language here, it is difficult to know precisely what he was thinking and what exactly his utterances meant. However, his switching back and forth multiple times indicated to me that he had an idea of what he wanted, but he was unable to bring his ideas together in a meaningful and concrete way.

As Nick waffled between which Expression he thought was correct, I conjecture he never took the time to purposefully think, "What exactly is my expression counting?" That is, he was caught up in examining the expressions and considering the counting processes, but he failed to clearly, explicitly connect the expressions and processes to the set of outcomes. Then, given the new Expression R, he never clearly articulated what the expression was counting. He had ideas about how issues of ordering the groups may have played into it, and I think he understood that he did not want to have the groups be distinguishable, but he could never decide which expression would give him what he wanted. As we see below, much of what he said was true (such as that "those 5 could also be chosen in Group 4 instead of Group 1, so that's why they divided by 4 factorial"). When it came to evaluating what each Expression was doing, though, he was simply not correct. His statement, "I said the groups weren't distinguishable," indicated a flaw in his thinking - he thought that his original answer did not count the groups as distinguishable, when really it $\operatorname{did}^{37}$.

\footnotetext{
${ }^{37}$ Incidentally, 9 other students also believed this to be the case in their initial attempts on the problem; they arrived at the answer in Expression $\mathrm{W}$ and interpreted the problem as I intended it.
} 
$\mathrm{N}$ : Because now that I've talked myself through this, when we did, like the way I did it, you're choosing people into the groups, where the groups don't matter, whereas this one, you're stating that the groups, you then have to divide by 4 factorial, because if you had these 5 that were in this slot, it's different if this was named slot 1 , slot, group 4 or whatever. Those 5 could also be chosen in Group 4 instead of Group 1. So that's why they divided by the 4 factorial. Was saying that each group was permanent, I guess you'd say, in that they were, each group was distinguishable... Where I said the groups weren't distinguishable.

E: Okay so the one on the right seems to indicate that the groups are distinguishable,

$\mathrm{N}$ : Yeah. And my answer is they're not distinguishable.

E: And you feel that it shows that they were distinguishable because it divided by 4 factorial?

$\mathrm{N}$ : Yeah, because there was, uh, 4 factorial different ways that you could order the 4 groups. Or 4 factorial ways that you could choose which group has which number.

In his work he had made a drawing; he had written vertical lines that separated space on the paper into four columns, and he put five $x$ 's into a column indicating a group in one of the columns. When he talked about having groups move around, he drew arrows from one column to another. I asked him about his drawing, and one interesting aspect of set-oriented thinking came up in his response. In order to explain that he did not want order to matter within the groups he gave a particular example of what one group might be. He noted that different permutations of 5 students in a group were actually the same.

$\mathrm{N}$ : You're putting 5 students in that group, so now these 5 students you can consider as one single unit within here [one of the columns]. So when we did this first initial analysis right here [the numerator of Expression W], that's already taking into account that these 5 can be any 5 . That if you did, if this was student $1,2,3$, 4,5 , if you chose them in that order, you put in $1,2,3,4,5$, it's the same as saying $2,3,1,4,5$ going into that group. 
I suggest that the underlined portion above indicates set-oriented thinking, where the set of outcomes he considered was combinations of 5 students within a group. Thus, at the level of determining whether the order within the groups mattered, he made a case for he wanted to count selections, not arrangements, of the students within each group. That is, he appealed to particular elements to indicate that order in the groups did not matter.

Nick then described how the different groups could be arranged, and again we see that he had the right idea - he noted that the " 5 could also be in this group, could also be in this group, could also be in that group." He seemed to have a sense of what he wanted to count (indistinguishable groups), but as we will see, he did not understand how his Expression $\mathrm{W}$ was not giving him that answer.

$\mathrm{N}$ : So you're taking out the permutations of selecting the group. But then, I was thinking, the other arrow was saying, well what if this was named Group 1. Then these 5 were in that Group 1. Well these 5 could also be in this group, could also be in this group, could also be in that group. But, it wouldn't matter to me, because these 5 are still just in their own group, they don't have a name, they don't have, they aren't distinguishable, they're just that group... So that's why they divided by the 4 factorial, that's saying that each group was independent and you were, each group was, had a name, a label, whatever you want to call it.

I asked Nick again about the group names he had used in his original work (the labels written above the binomial coefficients in his Expression $\mathrm{W}$ answer), but he dismissed the labeling as an organizational aspect of his work.

E: Okay, now in your solution you had written a group 1, group 2, group 3, group 4 above it,

N: Yeah.

E: What was that? 
$\mathrm{N}$ : Uh, that was just show that I was doing it by groups rather than students, that we have 4 different possibility - 4 different groups that the students could go into that wasn't anything for them to be - the groups weren't labeled or anything, that's just knowing that you have to split 20 things into 4 spots, rather than actual names.

As we discussed the problem further, he articulated that he believed Expression $\mathrm{R}$ was somehow indicating that the groups were different from each other, whereas in his Expression W, the groups he dealt with were not different from each other.

E: Okay and so the reason you don't like the answer on the right [Expression $\mathrm{R}$ ] is that you feel like, by dividing by 4 factorial it seems to be indicating that somehow that numerator had a permutation in it or something?

$\mathrm{N}$ : Yeah, and that the way, it seems like that the, in that [Expression R], it seems like it matters which of the students went where, so, um, basically you're stating that Group 1 is not equal to Group 2, not equal to Group 3. Where I'm saying that they're all, Group 1 is equal to Group 2 is equal to Group 3, because they all have 4 members, so it doesn't matter which group you go into...'Cause they're indistinguishable, they're still just groups of 5 people.

Again it seems that Nick saw that he wanted to count indistinguishable groups, but he could not attribute this idea to the correct expression. His language was a little imprecise here. It is not the case that the issue is whether the groups are equal to each other, but rather whether different arrangements of the groups (or different orders in which the groups were chosen) should be considered as the same or as different. He may have been trying to say something to that effect, but his language did not accurately reflect that idea.

In light of this situation in which he could not identify the correct answer, I asked him how he would respond if I told him that Expression R was actually correct. He said that the only way he could explain how Expression R was correct would be if someone using 
that answer had interpreted the problem differently. Below, we see again that he had the reasoning backward.

E: Okay. Okay great, so you want those groups to be indistinguishable. Now, just to maybe play devil's advocate, so let's say it turns out that the one on the right is the correct answer... So I'm wondering, can you reconcile that at all? Can you make sense of how that one could be correct?

$\mathrm{N}$ : This one?

E: Yeah.

$\mathrm{N}$ : Um, basically the only way that this one could be correct in my opinion is the person's understanding of what the question was asking. I think it was, yeah, it seems like depending on what they think the question was asking if they were, the question was asking for, like, back in elementary school when you actually had you are in Group 3, you are in Group 4,

E: Uh-huh.

$\mathrm{N}$ : Whether or not I think the question needed to state whether or not, uh, the groups were named or not... Is what determines which answer's right, I think. So it's just how the person would determine what the question was.

To finish our work on the problem, I tried to see if he could explain how his own answer might have imposed order and resulted in a permutation of the groups. And, as we see, this proposition did not make sense to him. And even given what appeared to be an understanding of the issues involved (namely that groups could have been ordered, and he needed to take care of that because he wanted the groups to be indistinguishable), he could not see how his answer was wrong.

E: I'm saying I think the one on the right [Expression R] is correct, and so how can you, um, justify that?

$\mathrm{N}$ : Yeah, so how was I missing the 4 factorial?

E: Right.

$\mathrm{N}$ : ...No, I can't see why mine would have a permutation of 4 factorial in it, because I'm dividing students into groups, I'm not trying to, 'cause in my answer I'm not dist - I'm doing nothing with the groups - all I'm doing is dividing students, I'm 
not dividing groups or anything.

E: Okay. Okay so the, your procedure of choosing 5 and then choosing 5 and choosing 5 and choosing 5, you're not doing anything to, to distinguish the groups or to order the groups at all.

$\mathrm{N}$ : No 'cause I have 20 students, I'm choosing 5 so I can't choose those 5 , so I'm getting, uh 15 ways to choose another 5 students, no matter which 5 choose here. I then have, I'm left with 15 to choose another 5. Yeah, no, I wouldn't - I don't see anything.

Nick was, in the end, not able to determine why the division by 4 factorial was a necessary feature of the correct solution to the problem.

4.2.3.3.3 - Summary. Unlike the other students discussed on this problem, Nick was ultimately unsuccessful at determining which answer was correct. While Nick seemed to be aware of what he wanted to count - namely divisions of 20 students into four indistinguishable groups of five - he was unable to do so successfully. While he even saw some of the major issues that might have been at play (such as the order of the groups playing a part somehow), he did not ultimately get the problem right, nor was he able to identify which expression was correct. It is hard to attribute a lack of success to any one thing, and I can only speculate on what happened for Nick. I do believe that he never successfully coordinated the counting processes associated with each expression with the appropriate set of outcomes. His one bit of clear set-oriented thinking, when he used a particular example, was to argue about the make-up of a particular group of 5, but he did not utilize this same type of argument to make a correct statement about the groups. Also, the back and forth discussion about which expression was correct suggested that he did not carefully and deeply consider what was going on in the problem. His work 
provided an illuminating example of someone who could not, ultimately, make sense of the role the 4 factorial played in the correct answer to the Groups of Students problem.

\subsubsection{4 - Other episodes}

I have discussed three students' work on the Groups of Students problem in detail. I now provide two other episodes that give a broader picture of student work on the problem. In particular, we will see how a question about the interpretation of the problem made a student change his work, and we will see one way in which a student identified a particular outcome that was overcounted by an incorrect answer.

4.2.3.4.1 - Daniel's interpretation of the problem. In this episode, we see an instance in which a student's work was affected by my questions about the interpretation of the problem. Daniel was the first student interviewed, and this episode had an effect on the rest of the subsequent interview episodes (in particular, I realized from this episode that questions about the interpretation could affect the correctness of an answer). After this episode, I always tried to make sure that I knew the students' interpretation of this problem.

Daniel had initially gotten Expression W as an answer, and he explained his thinking as choosing groups of 5 people from 20, then continuing to take groups of 5 until the people were gone. Then, I asked him how he interpreted the problem, and the following exchange took place. He explained that the issue of the groups being distinguishable was something he had considered initially when he read the problem, but then when he had actually done the problem, he had neglected to factor it in. 
E: Okay great. And um, let me ask one question. Can I ask, um, how you're interpreting it? So are you interpreting that to say that there are just sort of four groups of people, or that the groups of people are somehow labeled? So is there a Group A, Group B, Group C, Group D, or are there just four groups of people? Or did you think about that?

D: Oh, right. (Chuckles).

E: And so I guess I wonder initially how you interpreted the question and then which of those do you think that's answering? And this - you're doing great, by the way, this is exactly what I'm.

D: Um, right, yeah, no, I didn't even think about it. Um, yeah, right I did think about it a little bit. Um, right, it's the same thing, uh, because (chuckles) um, right, I could have...well, obviously this is if you labeled the groups. Um, so, um, right, I mean, I thought about it briefly, but uh, yeah, I just didn't uh, think to...

I then asked him to expound upon his statements above, and in his response he gave an insightful view into his thinking. In particular, he noted that there was a sequential nature to what he had done in generating Expression W, which is seen in the first underlined section below.

E: Yeah so tell me what you - what went through your mind and how you're thinking.

D: No no no, I thought about it briefly, but I just, I didn't really think about it at the end. And, but yeah, obviously, I mean, if you have some um, right, this would only be if they're labeled, because you do the first one, and then the second one, then the third one, then the fourth one... Because otherwise, you could - these count drawing, well, like, for example, you could draw, uh, a group of 5 people called A in your first draw, and then a group of people called B in your second draw, if A and B don't have any people in common. Um, but you could also draw B on your first draw and then A on your second draw, so if they're not labeled, then those aren't - I mean if you don't stick a 1 here and a 2 here, then these are equivalent. So because you have four groups, you have to divide by, uh, 4 factorial, which I guess should be the number of ways that you could permute those, uh, I mean the number of ways that you could what, yeah, if you have, yeah it is the number of ways of permuting any given four groups... Right, so, I guess you have to divide by, uh, 4 factorial. 
E: Okay, if I wanted them unlabeled then you would have to divide by 4 factorial?

D: Yes... Because it's the label that makes AB different from BA.

He then went to a particular example and called two groups of five people A and B, respectively, noting that the process in Expression $\mathrm{W}$ would count drawing A then B as different than drawing $\mathrm{B}$ than $\mathrm{A}$. And in the last line of the excerpt he noted that the labeling makes $\mathrm{AB}$ different from $\mathrm{BA}$. This episode is important because it shows that bringing the interpretation to his mind made him aware of a mistake he had made. And, too, his language suggests that he was able to coordinate his process with a set of outcomes. Even though $\mathrm{AB}$ versus $\mathrm{BA}$ represented just a partial example, his statements about two outcomes being different from each other indicates that he was thinking about outcomes in some capacity. His ability to work with the outcomes in that way arguably helped him to make sense of the difference between the two expressions and determine which was right according to the proper interpretation of the problem.

We thus see that my question about interpretation led him to reconsider the issue of whether the order of the groups mattered (and whether overcounting occurred). On two other occasions my question about interpretation caused students to change their work, but on the whole my questions about interpretation did not seem to have a direct bearing on students' work (they simply explained their interpretation and did not change their answers). Additionally, in this episode, Daniel's set-oriented thinking emerged as he considered partial examples that were counted more than once by an incorrect answer.

4.2.3.4.2 - Owen uses color coding to identify a particular instance of overcounting. In his work on the Groups of Students problem, another student, Owen, provided an example of 
a particularly nice way in which to identify overcounting. He had initially gotten a solution equivalent to Expression W (except he left off the last binomial coefficient), and his explanation highlights the sequential order in which he came up with the answer.

O: Ah, interesting. 4 groups of 5. Well, this one I mean, it seems pretty easy to pick the first group, you know, I have 20 people, choose 5... Great. Well, this one I feel kind of friendly with, 'cause then whoever I chose, I have 15 people left, I just choose 5... I mean, I might be wrong. But I only have 10 people left, and I choose 5. There's 5 people left, that's how I do it. That's how I'd count it. I don't know if that's right or not.

When we returned to the problem later in the interview, I gave him Expression R. As he noted in the excerpt below, he was immediately confused by the division by 4 factorial, and so he took some time to think about what might have been going on.

O: Okay so they basically have my solution, and then dividing through by some order.

E: Okay and why do you say some order?

O: Uh, 4 factorial is like, well it's like the number of ways to, uh, write down 4 things from a set or whatever, the number of orders. So, I mean I don't understand why they would divide by 4 factorial I guess... I just don't understand.

E: No it's good, it's good.

O: I'm immediately confused by that, and I would, to try to imagine what they were thinking, that's all you want me to - that's what you want me to do?

He noted, too, that he was unsure about dividing out, and that he had a tendency to overcount.

O: This is also something I've never been good at, figuring out when you're supposed to divide out options...I always get confused - I'm a big over-counter myself.

E: (laughs) Okay.

Owen clearly drew upon his previous experiences in the interviews, and we see this in the excerpt below. 
O: But I don't think it matters, like here, uh. Let me think about the. Let's do an example again.

E: Okay, great.

O: Because yeah this might be the same thing, like what if I picked, yeah I see, I see why they did it. What if I picked, okay, oh man I am bad at counting, okay.

E: You're not bad at counting, this is hard.

O: No no, I'm bad. Look, I think this student might be better than me, hold on. If I'm, let me walk through this students' thinking.

Indeed, at this point in the interview, he had just worked through the Passwords problem and had identified a particular element that was overcounted. So, when he said, "Let's do an example again," he was referring to his work on the previous problem, in which an example had helped him detect the overcounting. It appears that right after he started considering an example (no significant time passed in the video as he spoke in the excerpt above) he figured out what the issue might be. This suggests that the consideration of a particular outcome was beneficial for Owen.

Owen started to write an example, but he did not want to write out 20 objects, so he considered splitting up 8 students into four groups of two. He wrote down ABCDEFGH, and he wrote the corresponding solution that he would have done with 8 students,

$$
\left(\begin{array}{l}
8 \\
2
\end{array}\right) \cdot\left(\begin{array}{l}
6 \\
2
\end{array}\right) \cdot\left(\begin{array}{l}
4 \\
2
\end{array}\right) \cdot\left(\begin{array}{l}
2 \\
2
\end{array}\right)
$$

He then took different colored pens, and, as he explained the solution below, he underlined each binomial coefficient with a different color (pink, red, orange, and green, respectively). The corresponding drawing is seen in Figure 37. 


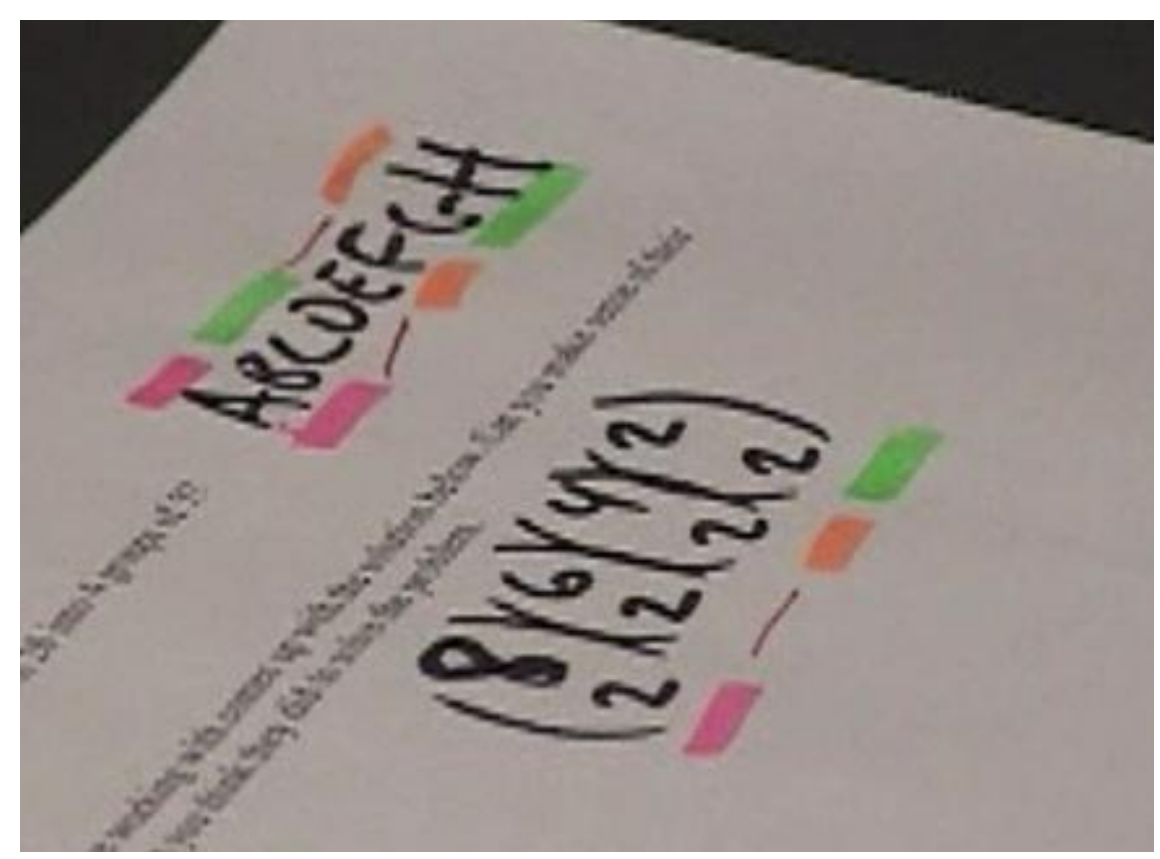

Figure 37

O: Okay look. So the same idea as before. I'm going to order my selections, because I think of them as happening in some order. So it's like this first [draws a pink line under $\left(\begin{array}{l}8 \\ 2\end{array}\right)$ ], and I'm going to do this second [draws a red line under $\left(\begin{array}{l}6 \\ 2\end{array}\right)$ ], this third [draws an orange line under $\left(\begin{array}{l}4 \\ 2\end{array}\right)$, and this fourth [draws a green line under $\left.\left(\begin{array}{l}2 \\ 2\end{array}\right)\right]$, right?

Then, in the excerpt below, when he said "I think it's the same idea" I believe he was referring to his work on the previous Passwords problem. He recognized that when he chose the groups of students to be $\mathrm{AB}, \mathrm{CD}, \mathrm{EF}$, and $\mathrm{GH}$, he could have chosen them in some order; the colors represented a particular order in which he chose that division of the 8 people. As he talked about choosing a pair of students, he drew a color under that 
pair (for example, when he said, "Then I pick C and D" he drew a red dash under those letters). He drew lines under $\mathrm{AB}$ then $\mathrm{CD}$ then $\mathrm{EF}$ then $\mathrm{GH}$ in that first example, and he drew $\mathrm{AB}, \mathrm{EF}, \mathrm{GH}, \mathrm{CD}$ in the second.

O: Okay, I think it's the same idea. What if, when I first chose 2 students, I picked, I'm going to make it really obvious, $A$ and $B$ [draws a pink line under $A B$ ]... Cool, then I have 6 remaining. Then I pick $\mathrm{C}$ and $\mathrm{D}$ [draws a red line under CD]. Oh, what if I have 4 remaining? Oh I'm going to pick these 2 [draws an orange line under EF, then draws a green line under $\mathrm{GH}]$.

He then noted that he could pick the same division of 8 people $(\mathrm{AB}, \mathrm{CD}, \mathrm{EF}, \mathrm{GH})$ in another way, and he drew colors above the letters to represent picking the pairs in a different order. This time, he drew AB first, then EF, then GH, then $\mathrm{CD}$. When he says "I could have picked CD or I could have picked EF" he referred to the different colors he drew below and above the letters, respectively.

O: Well, that's one way to count, pick 'em, right? Color, change the colors drawn above again. So you can put the pairs, you can pick the pairs in different orders... To make it obvious I'll draw the colors. Look, I'm just drawing the same exact pairs, different colors up here... To symbolize that if I did this, I could have picked him, this same pair twice, right, but second I could have picked CD or I could have picked EF.

E: Sure.

O: And then third I could have picked EF or GH...And last, $\mathrm{CD}$ or GH, the remaining pairs...Same exact pairs, counted twice here, counted only once here because you're dividing out the ordering of the pairs... So this is the order of the pairs, this is my overcounting, overcounting here, this is the number of ways to order 4 things, this way you get rid of those double counts.

Owen's work on this problem is noteworthy for two reasons. First, we see that he identified a particular outcome (or an example of an outcome in a smaller case) in order to address the overcounting issue and ultimately decide which expression was correct. He 
cleverly used a color coding scheme to indicate exactly how the same division of 8 students was actually counted more than once by the incorrect expression. His work here suggested set-oriented thinking, because he was able to focus on an example in the set of outcomes of the smaller problem in order to complete the problem correctly. That is, he related his counting processes to a set of outcomes, and doing so was beneficial for him. Second, he was influenced by a previous problem (in his case the Password problem). I point this out simply to note that he was affected by previous work, which likely influenced his decision to look for a particular example. Indeed, he went from total confusion about the 4 factorial to making sense of it in very little time, and his previous work seemed to have played into that; his previous success utilizing set-oriented thinking may have made him more likely to consider particular outcomes in his work on this problem.

\subsubsection{5 - Overall summary of the Groups of Students problem}

In the discussions above, we saw examples of ways in which students utilized particular sets of outcomes in order to identify a discrepancy between two solutions. In this problem, we saw the effectiveness of a smaller case and of systematic listing as Makaena utilized these strategies in order to recognize an overcount and make sense of different expressions, and Owen used similar strategies as well. I suspect that the smaller case allowed students to feel that the outcomes were more accessible, and they were more easily able to relate their counting processes with a particular outcome. We also saw a contrast between Zach's correct model of the problem as a sequence of sets of letters, and 
Nick's attempt to model the problem as a "stars and bars" problem. Zach also made a noteworthy observation about the tension of determining when one is "done" accounting for an overcount. Nick was ultimately unable to make sense of why the division by 4 factorial was a necessary aspect of the answer. Finally, we noted the importance of a proper interpretation of this problem, and this arose with the first interviewee. As a result of Daniel's interview, throughout the interviews I was always aware of making sure students interpreted this problem correctly. I now review and examine students' work on the Test Questions problem.

\subsection{4 - The Test Questions Problem}

The Test Questions problem states, "Suppose an exam consists of 10 questions, and you must answer 5 questions. In how many ways can you answer 5 questions if you must answer at least 2 of the first 5 questions?" In the discussion of the Test Questions problem, the common correct answer

$$
\left(\begin{array}{l}
5 \\
2
\end{array}\right)\left(\begin{array}{l}
5 \\
3
\end{array}\right)+\left(\begin{array}{l}
5 \\
3
\end{array}\right)\left(\begin{array}{l}
5 \\
2
\end{array}\right)+\left(\begin{array}{l}
5 \\
4
\end{array}\right)\left(\begin{array}{l}
5 \\
1
\end{array}\right)+\left(\begin{array}{l}
5 \\
5
\end{array}\right)\left(\begin{array}{l}
5 \\
0
\end{array}\right)
$$

will be referred to as "Expression R" (for "right") and the common incorrect answer $\left(\begin{array}{l}5 \\ 2\end{array}\right)\left(\begin{array}{l}8 \\ 3\end{array}\right)$ will be referred to as "Expression W" (for "wrong").

Twenty-one students attempted the Test Questions problem, and I revisited the problem with 17 of them. Of the initial responses, 11 got the problem right; 9 of them got the answer 


$$
\left(\begin{array}{l}
5 \\
2
\end{array}\right)\left(\begin{array}{l}
5 \\
3
\end{array}\right)+\left(\begin{array}{l}
5 \\
3
\end{array}\right)\left(\begin{array}{l}
5 \\
2
\end{array}\right)+\left(\begin{array}{l}
5 \\
4
\end{array}\right)\left(\begin{array}{l}
5 \\
1
\end{array}\right)+\left(\begin{array}{l}
5 \\
5
\end{array}\right)\left(\begin{array}{l}
5 \\
0
\end{array}\right)
$$

2 got the correct answer

$$
\left(\begin{array}{l}
10 \\
5
\end{array}\right)-\left[\left(\begin{array}{l}
5 \\
1
\end{array}\right)\left(\begin{array}{l}
5 \\
4
\end{array}\right)+\left(\begin{array}{l}
5 \\
0
\end{array}\right)\left(\begin{array}{l}
5 \\
5
\end{array}\right)\right]
$$

3 got the incorrect answer $\left(\begin{array}{l}5 \\ 2\end{array}\right)\left(\begin{array}{l}8 \\ 3\end{array}\right)$, while 7 students gave some other answer.

Additionally, when students evaluated the alternative solutions presented to them there were three times in which students suspected that differing solutions were the same, and in all three of these instances, students were ultimately able to reconcile this difference.

\subsubsection{1 - Joshua's work on the Test Questions problem}

4.2.4.1.1 - Overall description. Joshua arrived at the correct answer, Expression R. As he did so, he did not appear to consider the set of outcomes (utilizing the relationship between counting processes and formulas/expressions), although he did successfully utilize the case breakdown. As we returned to the problem, I gave him Expression W, and he felt like it made sense. He talked through what the process represented by Expression W did, and he felt that it should be equivalent to his work (Expression R). He then compared the two expressions numerically and found that they were different, and he tried to reconcile the difference. In spite of significant prompting from me, he said that he did not understand why Expression W was incorrect. Then, I explicitly told him that his answer was correct and that the other was too big, and I asked him if he could find a particular instance of something that was counted more than once by Expression W. With 
this prompt, he was able to identify a particular outcome in order to explain why Expression W was too big (coordinating the counting problem with the set of outcomes to do so). This episode is another example in which a student thought two processes were the same, and then drew upon the set of outcomes (appealing to a particular instance) to explain why they were, in fact, different.

\subsubsection{2 - Detailed account}

4.2.4.1.2.1 - Joshua arrives at the correct answer, Expression R. As he worked on the problem initially, Joshua arrived at the correct answer. He broke the problem into cases according to whether $2,3,4$, or 5 of the first 5 problems were answered, and he talked about each product within each case as a "complete event."

J: Um, then what I do, I will do is, uh, from the first group, uh, I will pick up 5, 2 questions from $\mathrm{it}^{38}, 5 \mathrm{C} 2$, the rest of the 3 questions will automatically, I need to do it from the other groups, I don't worry about that much... Uh, then, the uh, uh second question, second case I will think about this like $5 \mathrm{C} 3$, in the first group picking up 3 questions. And, uh, third one is $5 \mathrm{C} 4$, And fifth one is $5 \mathrm{C} 5$, all the 5 questions in the first group I'll do it. But, the thing is, when I do the first 5 questions, $5 \mathrm{C} 2$, that means from the rest of the 5 I need to do 3 of them, which is $5 \mathrm{C} 3$. This is my complete event... Then, from the first group, if I do the $5 \mathrm{C} 3$ questions, then from my second group I'm doing $5 \mathrm{C} 2$. Then comes $5 \mathrm{C} 4$, the

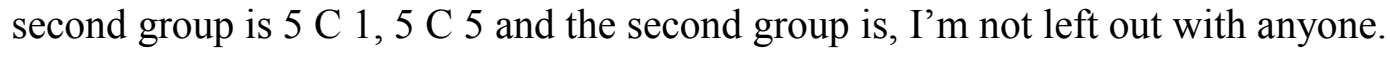

His language above indicates that he completed a counting process to arrive at an answer, but he did not consider the set of outcomes. I suggest that in terms of the model, his work

${ }^{38}$ Joshua tended to write his binomial coefficients as ${ }_{5} C_{2}$ instead of $\left(\begin{array}{l}5 \\ 2\end{array}\right)$. 
above was entirely in the relationship between counting processes and formulas/expressions, and he did not draw upon the set of outcomes at all as he solved the problem initially (Figure 28 above). This is an example in which a student arrived at a correct solution by working exclusively within that top relationship.

4.2.4.1.2.1 - Joshua feels that Expression $R$ and Expression $W$ are equivalent. We revisited the problem again; I gave him Expression $\mathrm{W}$, which is too big, and we see below that he made sense of the alternative answer and felt like it should have been equivalent to his original answer. We see that it was natural for Joshua to examine the expression and make a conjecture about what counting process could have been reflected by that expression. When I asked "How would you make sure that they're equivalent?" I expected that he might say something about numerical calculation, but instead he reconfirmed his thinking and talked through the process that Expression W represents.

$\mathrm{J}$ : Um, okay. Uh, yeah, this [Expression W] makes more sense when compared to this [Expression R] because what you have done is like, you picked up 2 questions from the first, uh, 5 questions, from the rest of the 8 questions, which are left out, you picked up 3 of them. So, I, I think this [Expression R] is, uh, this needs a step by step by step thing.

E: Okay so the process [in Expression W] makes sense?

J: Process makes sense, yeah.

E: Um, do you think they're equivalent?

$\mathrm{J}$ : Yeah they are equivalent, both of them.

E: Okay, okay so, how would you make sure that they're equivalent, like how could you check?

$\mathrm{J}$ : Um, because the process with which, um, we are, here, my, my problem of $5 \mathrm{C} 2$, $5 \mathrm{C} 3,5 \mathrm{C} 4,5 \mathrm{C} 5$, uh, is taken care with this $8 \mathrm{C} \mathrm{3}$, The rest of them, and, minimum two groups from the first group is taken care of with the first one [5 $\mathrm{C}$ 2]. So it [Expression W] makes a lot of sense. 
I then asked him to numerically work out each expression, and he found that Expression R yielded 226 while Expression W yielded 560. Joshua seemed puzzled by this, and he maintained that they both "conceptually make sense." As he discussed it more, he seemed to be even more certain of Expression W than his own Expression R.

$\mathrm{J}$ : Your thing makes sense [Expression W], and my thing also makes sense, so. $5 \mathrm{C} 2$, 5 C 3 times 5 C 2. I'm not... Conceptually both of them,

E: Makes sense?

J: Yeah make sense. But uh, I don't know then um, this [Expression W] makes for sure, like sense to me, because I'm picking up 2 questions from the first 5 , then the rest is all 8, so.

Again, in terms of the model, Joshua was still functioning within that top relationship between counting processes and formulas/expressions. It is noteworthy that both processes made sense to him, and he was at a point at which he could not make sense of why the processes might yield different answer.

\subsection{3 - Despite prompts, Joshua does not write down a particular instance of what he} is trying to count. Then, Joshua worked for about five minutes more, and I gave a couple of significant prompts during this time. As we will see, these prompts were not particularly useful, and ultimately he was not sure why the two answers would be different (and why Expression W would be wrong). First, I prompted Joshua by asking if he could come up with something that was missed by his expression or counted too many by Expression W (because his was smaller numerically).

E: So can you maybe think about, you know either come up with something that does get counted,

$\mathrm{J}$ : In mine?

E: Well yeah, maybe either something, I mean, yours is smaller than theirs, right? 
J: Yeah.

E: So either, you know, yours is missing something,

J: Yeah.

E: Or theirs is counting something too many times, so can you try to, you know, think of a particular solution, either that they're counting too much or that yours misses?

In response to this prompt, he mentioned one case, as seen below. It was not clear whether this line of reasoning was productive for him, and I prompted him again before he could reflect more on this idea (in retrospect I would have given him more time).

$\mathrm{J}$ : In how many ways can you answer 5 questions if you must answer at least 2 of the first 5? At least 2 of the first 5. Okay the thing is, I'm not sure about the, mathematically if I think about it, or if I compute the value, uh, there is one case for sure which will arise out of the whole of it that is like, uh, from the first section of 5 questions, all 5 questions I will do it, which is one way of doing it.

In the moment, I felt that his discussion above was not productive, and I thus prompted him to write down some particular examples of what was being counted.

E: So will you do me a favor and maybe write down a couple of particular examples that is getting, that are getting counted? So like what's an example of what you're trying to count?

$\mathrm{J}$ : Here the things which I'm trying to count is like, $5 \mathrm{C} 2$ is from the first, uh, 5 questions I'm picking up two, Uh, in this next 5 group that, that means, uh, uh,

E: Well so just give me one example of that. So say you have questions number 1 though 10. Like what's an outcome of that process?

$\mathrm{J}$ : Um, since I know there are two sections for sure, First section A has 5 questions, section B has 5 questions for sure... Now, I'm certain that I'm picking up two questions from the first group, and I'm certain that I'm picking up three questions from the second group.

E: Uh-huh.

J: Then 5 C 2 times 5 C 3 makes sense for me over here. Nothing wrong with that.

I gave the above prompt for a couple of reasons. First, I felt that such a prompt could help Joshua see the error in Expression W (in fact such activity had, at times, proven useful as 
a means by which to help students identify overcounting). Second, I had not seen evidence in his work thus far that he had considered any instances of what he wanted to count, and I was curious if he would be able to do so. As the excerpt above shows, I asked him to write down particular examples, but he did not initially do this. He described what he was doing, but he did not provide an instance of what he was counting. I therefore asked him again for a particular instance (even suggesting he consider numbering the questions), but again he did not write down a particular example.

Then, since Joshua still had not written down a particular outcome, I asked him yet again, and he provided me with a particular instance of what had been counted. After he arrived at $\{1,2,6,7,8\}$ as something that would have been counted "in the first one" (Expression R), I asked him to give an example of what Expression $\mathrm{W}$ might have yielded. While he did note that he could have chosen questions 1 and 2 in the first term of Expression W, he did not go on to articulate an actual complete example of an outcome of Expression W.

E: ...What's a set of questions that would be gotten?

$\mathrm{J}:$ In the first one?

E: Yeah.

$\mathrm{J}$ : Uh, I would have taken 1 and 2, in the second 1, 2, and 3, or 6, 7, 8 .

E: 678 , okay, okay cool....

E: So 1, 2, 6, 7, 8. Good and then what's an example for the 5 choose two times 8 choose 3 .

$\mathrm{J}$ : Uh, let us say I've picked up from the first 5 two questions, let us assume the rest of the 8 , uh, the rest of the 8 questions, let us say, let us assume I picked up one and two, question number one and two... I'm left over with 3 onwards til 10, I can pick up any of the 3 questions from those, so, that's how I look at it. 
Our discussion continued, and Joshua, in considering Expression W, noted his options for $\left(\begin{array}{l}5 \\ 2\end{array}\right)$. Ultimately, however, he was not able to see what was wrong with the incorrect expression. In the underlined portion below, we see that he felt Expression W was fine, and he was "not sure" what was wrong with it.

J: So that, that 5 C 2 can be $12,13,14,15$, E: Yup.

$\mathrm{J}$ : Or 23, 24, 25, 33, 34, 35, in that way it can be anything, that is taking care of that.

E: Yeah.

$\mathrm{J}$ : Automatically $8 \mathrm{C} 3$ will pick up the rest of the questions other than those 2 questions... Yeah I'm not able to get, this [Expression W] is fine, what went wrong with it, that's, yeah, I'm not sure.

At this point, some more time had passed, and I felt he was at a loss of how to explain the error in Expression W, so I intervened again. I told him that his initial answer was correct and that Expression W was too big, and I asked if he could find a set of questions that would get counted more than once.

J: More than once. Yeah, oh, yeah I got it. Now let us say I picked up question number 2 and $3 \ldots$ There is a chance that, uh, 1 and $4 \ldots$ Questions I would have picked up, um, okay, now it makes sense.

E: Yeah, you can write it down if you want.

$\mathrm{J}$ : Now, let us say I picked up question number 2 and 3 from the initial 5. Then, um, the questions 1 and 4 I answered it, as the other two, so let us say 2 and 3 I picked it out, then I went for 1 and 4. Let us say. In the other case, if I would have picked up 1 and 4, in the rest of the combinations of 8, this 2 and 3 are again present, if I would have picked up this 2 and 3 again, it's a repetition which is happening over here... So that's the reason I think I would have got additional stuff over here [Expression W]. Now that's what is the drawback is I guess of the solution over here [Expression W]. 
Above we see that Joshua went on to successfully identify a set of outcomes $(\{1,2,3,4$, $5\})$ that was counted more than once. His discussion suggests a coordination of sets and processes - he was able to talk about how the respective processes could each generate the particular outcome.

Then, to hear his explanation again, I had him repeat his argument, and we see that he was able to explain why the overcounting occurred. He said that I had given a "good hint," and so clearly my prompt to identify a particular set of questions that was overcounted by Expression W had an impact on him. I think, though, that this suggests that an attention on a particular element of the set of outcomes can be useful in making sense of processes and ultimately helping to understand what a particular process does.

E: Okay so maybe just one more time, sum up what, what you found, why that...

$\mathrm{J}$ : Yeah, yeah the thing is, if I do it this [Expression W] way, let us assume I pick up question number 1 and 2 from the first group. Then, um, there's a chance that, from $8 \mathrm{C} \mathrm{3}$, there's a chance that I may pick up question number 1 and 4 , uh the other case which I'm taking now is, uh, picking up question number 1 and 4 as my $5 \mathrm{C} 2 \ldots$ In $8 \mathrm{C} 3$ if I would have picked up 2 and 3, this group and this group would have been the same. Or, let us say 1, 4, 5, over here, let us assume over here if I would have picked up 2, 3, 5, that would have made my 5-set questions... So this would have been a repetition, yeah.

E: Awesome.

J: Yeah, that's how I would have, that's the additional stuff which is present in this [points to 560].

E: Okay. Okay good job. Cool.

J: Initially it makes sense, but that's a good hint which you have given.

4.2.4.1.3 - Summary. Joshua answered the problem correctly initially, but when we revisited the problem he could not immediately see why Expression W was too big. When I prompted him to write down particular instances of what he was trying to count, 
this did not seem natural for him, and in fact it took me repeating the prompt multiple times before he wrote down a particular instance. Even after some prompting, he felt that the two expressions should be equivalent, and he could not justify an error in Expression W. It was, finally, a very direct prompt to write a set of questions that was overcounted by Expression W (which he did successfully) that helped him to see why the alternative answer was incorrect. When Joshua did write down the particular example, he seemed to be able to coordinate the counting processes with the outcome he came up with. I believe this coordination helped him ultimately make sense of the overcounting issue. It seems that while Joshua had initially not drawn upon sets of outcomes in his work, through my prompting he did consider identifying particular outcomes. Ultimately, this enabled him to recognize why the processes differed. I conjecture that appealing to the set of outcomes helped him to understand elements of the problem that examining the relationship between counting processes and formulas/expressions alone could not.

\section{2,4.2 - Kristin's work on the Test Questions problem}

4.2.4.2.1 - Overall description. Kristin got the problem correct initially, arguing through a case breakdown and arriving at Expression R. As she explained her solution, she noted that order did not matter, and so she wanted to work with combinations. When I asked her to explain why order did not matter, she explained her work by providing some examples of two different sets of five questions that she did not want to consider as the

same. While I interpreted it as a good thing that she went to particular examples, the way that she thought about her examples was not correct. When she described why order did 
not matter in her expression, it was clear that she was not correct in her thinking - two solutions that she wanted to count as the same should actually have been counted as different. When we revisited the problem, I gave her Expression W, and she made sense of what it was doing. She thought, though, that it missed things - she was not convinced that the $\left(\begin{array}{l}8 \\ 3\end{array}\right)$ actually captured all of what her cases did. As she continued, it became clear that the picture she had been working with (the incorrect way of thinking about the order mattering) was still in her mind, and it ultimately prevented her from truly understanding what the alternative expression was doing. She was thus, in the end, unable to reconcile which expression was correct and why. She felt confident in her own answer (and she was correct), but she could not explain why the other expression was incorrect.

\subsubsection{2 - Detailed account}

4.2.4.2.2.1 - Kristin clarifies what she tries to count and addresses an issue of order. It took some time for Kristin to orient herself to the problem, as she wanted to clarify what was being asked. Once she did, though, she set about solving it, and she decided that she would utilize cases. In her discussion we see that she noted that "order doesn't matter," a statement that we would address subsequently in the interview. She also described why she multiplied and added, and the "or" situation indicated to her that she should add. So after her initial work she had written Expression R as her answer, and she seemed fairly confident in her work. 
K: So I have 10 questions, and I must answer 5 of them, so 5 have to be answered. And then they want to know in how many ways can, in how many ways can you answer 5 questions if you must answer at least 2 of the first 5 questions? So I have to answer 2 of the first 5 .

So, from just reading that last line I know I have that constraint, so I'm going to have cases I think. So I know I have to answer 2 of the first 5 questions. So I can either answer 2 of the first 5 and 3 of the second 5, 3 of the first 5 and 2 of the second 5, 4 of the first 5, 1 of the second 5, and all 5 from the first 5. So, in how many ways can you answer those 5 questions? So, order doesn't matter, so I would use my combination.

So I'm going to have cases. I'm going to have, you can do 2 and $5-2$ and 3, you can do 3 and 2, you can do, uh, 4 and 1 or 5 and none. So I'm going to have four cases. So if I have 5 in the first one, and I only pick 2 out of those 5 I'm going to have 5 choose 2 . And then multiplying because they depend on each other, by, I have 5 questions in the second half, so 5 choose 3 of those to answer. And then I'm going to add it, since it's, I can have this case or this case or this case, and I'm going to do 5 choose 3 and 5 choose 2, plus 5 choose 4 and 5 choose 1 plus 5 choose 5 and five choose 0 .

Kristin thus arrived at the correct answer. While she did utilize a case breakdown, the extent to which she used sets of outcomes is not clear.

4.2.4.2.2.2 - Kristin incorrectly identifies a particular outcome. Then, I asked Kristin to say a bit more about how she determined that order mattered. Her response turned out to be quite important for the rest of the problem, and I want to describe carefully exactly what she wrote as she gave the response below. As she said "So I have 5 questions, and answering these 2 would be the same as answering those 2 , or these 2 ," in the underlined section, she drew five dashes. Then, as she said, "answering these two" she wrote $x$ 's in the second and third dashes. As she said, "would be the same as answering those 2," she wrote $x$ 's in a row above in the fourth and fifth spots. Then, as she said, "or these two," 
she wrote two more $x$ 's above the second and fifth spots. Thus she had a diagram like the one in Figure 38 below, where she wrote each row in subsequent stages.

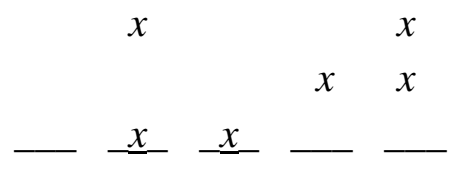

Figure 38

E: Okay, cool. Great, now I was going to ask you a couple of questions. Um, oh at one point you said, oh yeah you decided whether order mattered or not.

$\mathrm{K}: \mathrm{Mm}-\mathrm{hmm}$.

E: And you decided that it didn't, so can you say any more about what that thought process was for you, or what made you decide?

$\mathrm{K}$ : Well, I kind of pictured in my head, of the first, only of the first 5, 'cause, They would be the same... So I have 5 questions [draws 5 dashes], and answering these 2 [draws $x$ 's in the second and third dashes] would be the same as answering those 2 [draws $x$ 's above the fourth and fifth dashes], or these 2 [draws $x$ 's above the second and fifth dashes], because I don't care which ones I'm answering, as long as I answer 2 of those $5 \ldots$

E: Okay, okay so that's kind of what you were thinking of when you decided to go with the combination formula.

It is noteworthy that, in her explanation of why she did not think order should matter, she appealed to a particular example. In fact, she listed three possible ways of answering two of the first five questions, and she noted that she considered each of those outcomes as being the same. Generally, the approach of identifying certain outcomes as "the same" can be a useful strategy when explaining why order does not matter. There is, however, a significant problem with the diagram she drew, and her picture indicated that she was not properly thinking about the issue of order in the problem. She was correct that order does not matter as she chooses the test questions (and indeed her combination formula is 
correct). The issue in this problem is not which test questions were chosen, but rather the order in which they were chosen. That is, order is irrelevant in the problem because choosing to solve problem 1 , then 2 , then $6,7,8$ would be the same set of five questions as if someone had answered 2, then 1 , then $8,7,6$ - the order in which they were chosen does not matter. As we see, however, this was not how Kristin took order to matter. Instead, as she explained above, her work meant that any of the subsets of problems are actually essentially the same. The way she described her diagram suggested that she would consider answering $\{1,2,6,7,8\}$ to be the same as answering $\{2,4,6,8,9\}$. Now, I do not really believe that this was what she intended - if this issue had been clarified, and she had realized what her explanation here suggested, I think she would have been surprised. And, in fact, her diagram does not align with what her correct answer (Expression R) actually says; but the point is that the diagram became her model for how to describe order, and it would later end up being problematic as she tried to make sense of the alternative answer. During the interview, when I saw how she understood her diagram, I suspected that it would be problematic if this was indeed her conception of why order did not matter in the choosing of the test questions. However, I also wondered if she had not misspoke (and perhaps did not understand what her diagram actually said). I did not correct her (partly because I wanted to see what she would do naturally), but I was curious to see if perhaps the same issue would arise subsequently in the interview, and we will see later that it did.

I then asked Kristin about why she went to cases, and she said that the "at least" language was a trigger for her. I also asked her why she multiplied and added when she 
did, and it seemed, too, as though "and" and "or" were important words that indicated multiplication and addition, respectively. This appeal to key words was typical of Kristin's work.

E: What was it that made you think to do cases?

$\mathrm{K}$ : So, um, there's a lot of information in this question, so after I went through and wrote down - I wouldn't have thought cases until I wrote what I wanted down, and then I saw I have this constraint that 2 of the first, at least, at least 2 of the first 5 must be answered, so I know I can either have 2, 3, 4, or 5. If it had said exactly 2 , then I wouldn't have thought cases.

E: Okay. Okay. Um, great, and then why, and I think you said this again, but why did you add when you added and multiplied when you multiplied.

K: So, I multiplied 5 choose 2 and 5 choose 3 because they kind of rely on each other, I can have 2 from the first and 3 from the second, so there's the "and." And then I can have that possibility or I can have 3 from the first and 2 from the second, so there's the "or," which is where I add.

In terms of the model, I have two points to make about her work in this section. First, Kristin's incorrect articulation of different outcomes shows a way in which the relationship between counting processes and sets of outcomes can "break." That is, she was correct in wanting to articulate a particular outcome and to use that to make an argument about order mattering, but she was not able to correctly identify outcomes that should be the same or different from each other. So while she did not neglect this relationship, she did not correctly forge the relationship. Second, Kristin's attention to key words like "and" and "or" to determine multiplication or addition, respectively, suggests to me that she has a particular view of counting that puts a premium on the top relationship of counting processes and formulas/expressions, and that she does not seem to see the value in utilizing sets of outcomes. 
4.2.4.2.2.3 - Kristin's incorrect notion of an outcome creates further problems. In the second interview, we revisited the problem. Kristin talked back through her work, explaining her case breakdown, which had yielded Expression R. I asked her about order mattering (perhaps wondering if she might have changed her previous, incorrect description), but she again provided the same explanation for why order mattered. As she gave her explanation seen in the excerpt below, she again drew out five dashes. And as she described "the first two" and "the last two," etc. she drew $x$ 's above the respective dashes. This revealed the same mistake discussed above.

E: At some point I think you had said something about order not mattering, K: Right.

E: And so you went with combinations. Can you say a little bit more about that?

K: So I guess I kind of pictured it in my head, if I have, these are my first 5 questions...And I only want to have 2 of the first 5 for the first case, I could have the first 2, I could have the second - the last two, I could have the first and the third. It doesn't matter which two I pick, as long as I pick 2.

She talked a bit more about whether or not order mattered, and the excerpt below gets at how she thought about order. She discussed the numerical expressions for permutations and combinations, and she talked about which type of operation yielded a smaller number. She did note that the combinations have fewer possibilities, but her argument seemed to be rooted more in the numerical manipulation of the formulas than in a consideration of the set of outcomes. She did not talk clearly about wanting different objects or her set of outcomes being the same or being different.

K: So that to me means that order doesn't matter and I know that, with order not mattering you do a combination not a permutation... But I always get confused with those two, which one is the one that has order matters, and which one is the 
one that doesn't.

E: Okay, and what. Can you say anything about how you go about deciding whether order matters or not. Or which, to use the permutation or combination. You said you have trouble with it,

$\mathrm{K}$ : Um, so I kind of think back, so 5 choose 2, that would be, um, 5 factorial over 5-2 factorial, so 3 factorial, over 2 factorial for a combination, Whereas if it was a permutation I'd have 5 factorial just over 3 factorial. So I know that this one is going to be the smaller... So that's how I kind of remember order matters. Because if order matters, they're going to have less possibilities than if order didn't matter. I said that wrong, So combination has less possibilities, meaning order doesn't matter. Whereas a permutation order matters, so you have more possibilities? Yes.

E: Okay, so which has more possibilities, would you say?

K: The permutation.

I then asked her to examine the alternative solution, Expression W. As she did so, she drew out a similar diagram as she had before (Figure 39 below). As she said the portion underlined below, she drew out ten dashes, in which the first and second five were separated by a vertical line, and she filled in the $x$ 's in the following way. While she had a diagram at her disposal, and I think she was trying to articulate particular instances of what she was trying to count, her understanding of the outcomes was not correct. My interpretation is that because the diagram was fundamentally flawed, examining the diagram did not help her to make sense of how overcounting occurred in Expression W.

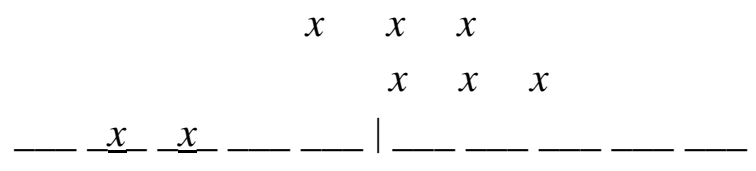

Figure 39

In fact, we see below that her instinctual reaction to Expression $\mathrm{W}$ was that it was too small, that it did not take into account all of the possibilities. 
$\mathrm{K}$ : You must answer 5 questions. In how many ways can you answer (inaudible)? So 5 choose 2 , and then 8 choose 3 . Oh, I see. So they did, they first picked their two from their first, um, first 5 questions... And then they were saying that there are 8 questions left to choose from, and you need to pick 3 of them. But if I were looking at that, I would draw it out so I have ten questions, so this is like my first 5 and second 5 [draws ten dashes]... And so they're saying 5 choose 2 for the first 5 , so I, just any 2, so the second and third let's say [draws the $x$ 's in the second and third dashes]. And then you have 8 left for the 3, but so you could have 3 in the second 5 [draws the three $x$ 's above the fifth, sixth, and seventh dashes], you could have 1 in the first 5, 2 in the second 5 [draws the three $x$ 's above the fifth, sixth, and seventh dashes]. So to me that wouldn't take into account every single possibility.

E: Okay.

$\mathrm{K}$ : Or would it double count? And you can have all 3. Mmm. I think they don't take into account every possibility. 'Cause in here they can have, they're saying you might have all 5, and then you might have all 5 in the first one, and you might have 2 in the first, and 3 in the second. But I, to me it just seems like it should be broken up.

She wavered for a bit (“or would it double count?") but by the end of this exchange she suspected that Expression W missed some possibilities. Also, while she wanted the problem to be broken up ("to me it just seems like it should be broken up"), I interpret that this was based more on prior counting experience than on a mathematical argument about why a case breakdown would be necessary or desirable.

Being unsure of whether Expression W was too big or too small, she then proceeded to look at both expressions numerically; she attempted it by hand for a while, manipulating the expressions, but then she turned to a calculator. She explained why she was doing the numerical comparison, and she said she was checking because she suspected that Expression W was too small and was missing some things.

E: And so, and the reason you're calculating these out is why? 
$\mathrm{K}$ : Because I don't think that they took into consideration the separate cases. You could have 2 ... I mean they did take it into consideration how you could have 2, 3 , 4 , or 5 in the first 5 , but in the way they counted it, they, how do I explain it. In the way they counted it, they're kind of lumping it into one thing... Whereas you have to split it up... So I'm checking it by, so I think that they haven't counted enough possibilities, because the 8 choose 3 doesn't justify if you have 2, 3, 4 or 5 in the first 5, so if I were to do it out, I'm pretty sure this [Expression R] would be a bigger number than this [Expression $\mathrm{W}]$.

E: Okay and that's what you're checking now?

$\mathrm{K}$ : Yes.

E: Okay, perfect.

When she arrived at the numerical difference, she found that Expression $\mathrm{W}$ was too big, and so she said that "they overcounted." As mentioned above, she appealed to cases because she knew from experience that she should do that. She did not give a solid explanation for why overcounting might have occurred.

K: So they over-counted.

E: Okay so by comparing those two numbers, um, you said they overcounted. How did you know that, what does that tell you?

K: That they're wrong.

E: That they're wrong, okay (laughs).

K: Assuming I'm right... But, I don't know how I know to break that up into cases, otherwise I know that you over-count. I think that's just from doing a lot of problems with the at least or at most.

Then, Kristin tried to consider how Expression W might be overcounting, and again she turned to her (flawed) diagram (Figure 40 below). As she looked at her diagram she considered two different possibilities that might have been generated by Expression W. In the $\left(\begin{array}{l}5 \\ 2\end{array}\right)$ part of Expression W, she put $x$ 's in the second and third spots, drawn below. 
She then considered the situation in which, in the $\left(\begin{array}{l}8 \\ 3\end{array}\right)$ of Expression W, she chose " 1 in the first five and 2 in the second five," and she added another row to her diagram (with $x$ 's in the fourth, sixth, and seventh spots). Then, as she made the statements underlined below, she circled two of the outcomes, as below. In particular, she was concerned with whether the two circled possibilities would be considered as the same or different. The way she had been explaining the diagram to this point, she would have considered them the same (because they represented answering 3 in the first 5 and 2 in the second 5).

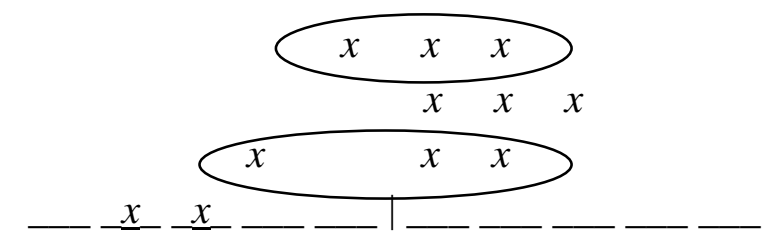

Figure 40

K: Because I feel like they have, they have like this case, with the 2 , they're choosing the 2 and the 5 first, and then let's say they have 1 in the first 5 and 2 in the second 5... And then they also have this case, which would, you still have 3 in the first one. But, they're [referring to the two circled possibilities] different. Or those are the same, because order doesn't matter. I'm confusing myself thinking about this.

E: Okay that's okay, so, but you found the difference there, makes you feel like they overcounted. And why do you say that, why does the bigger number indicate that?

$\mathrm{K}$ : Well they have a lot more possibilities than I got... So somehow that 8 choose 3 double counts things. But I don't know how.

E: Okay and what do you mean double counts?

$\mathrm{K}$ : It's counting like these 3 , so 3 in the first and 2 in the second, and also if you put the third one in a different first spot, And the same second 2 in the last 5 ? But that doesn't feel right, Explaining it that way. So I don't know.

It did not seem as though Kristin completely understood what was happening in the diagram, and whether Expression R or Expression W considered the circled outcomes as 
distinct. Even in spite of her diagram, and her consideration of particular outcomes, we find that she was mistaken, and that ultimately her consideration of the particular outcomes was not helpful for her. Additionally, her language above showed that she was ultimately unsure of how Expression W was too big and had more possibilities than her answer.

We continued to discuss the problem, and again she referred to the diagram and showed that she did not have a correct understanding of the outcomes of her counting procedure. As she made the statement below, she drew the same diagram again, drawing $x$ 's above the respective dashes.

K: So to me I was saying that order doesn't matter. I did say that, right?

E: That's right.

$\mathrm{K}$ : So to me that would mean, in my case picking the second one and the third one and then picking the first, second and third of the second 5 [drew $x$ 's above the second, third, sixth, seventh, and eighth dashes] would be the same thing as picking the first one and the second one in the first two and say the third fourth and fifth in the second five [drew $x$ 's above the first, second, eighth, ninth, and tenth dashes]. Whereas in this case [referring to Expression W], those are different.

E: Okay, and how so, how are they different in that case?

K: Well he's, I mean they might not necessarily have intended them to be different, but the counting that they're using is different - makes them different... Because they have the 5 choose 2, so that's totally fine, saying you can pick any 2 , and that doesn't double count, the 5 choose 2 . But the 8 choose 3 is counting, if you have, say you pick the second and the third one, and then the first three of the second half in this one... They're saying is different than picking the second and the third and then the fourth fifth and sixth in the second half. So it's overcounting by a lot. Because then it would be the same as, like, those 3 or any 3 .

E: Okay so it's counting something as different that you want to count as the same.

K: Right. 
4.2.4.2.3 - Summary. In Kristin's work on the Test Question problem, while she initially got the problem correct, we saw that early on she used a diagram to articulate her understanding of an outcome of her counting process. Her understanding of what made outcomes different from each other was incorrect, though. Specifically, she considered sets of five questions to be different only if they represented different numbers from the first and second halves of the test. This is a noteworthy example because her work indicates that she did consider the set of outcomes. And in fact when she wrote down instances of what she was counting, the activity suggested an ability to coordinate between a process and the set of outcomes it generated. Kristin never properly articulated the correct set of outcomes associated with each processes, though, and this kept her from being able to make sense of what Expression W was really doing, which in turn kept her from identifying how she might have overcounted. Kristin's work provides a useful view of a potential consequence of an incorrect coordination of a process and the outcomes it generates. Additionally, as with her work on the Passwords problems, there were times in which Kristin seemed to be focused on key words, on her past experience, or on intuition to solve the problem.

\subsubsection{3 - Aiden's work on the Test Questions problem}

4.2.4.3.1 - Overall description. From the start of his work on this problem, Aiden's language and activity showed that he considered the set of outcomes. In particular, he referred to the total space of set questions, and he provided some particular instances of what might be contained in that space. He then correctly utilized a total minus bad 
strategy, subtracting from the total sets of 5 questions those sets of questions with fewer than 2 chosen from the first 5 . When we revisited the problem, he initially misread what the alternative expression said, but he ultimately made sense of it. He was able to identify a particular outcome that had been counted more than once by the incorrect answer, which helped him to understand how overcounting had occurred.

\subsubsection{2 - Detailed account}

4.2.4.3.2.1 - Aiden utilizes a total-minus-bad strategy. Aiden first talked about the entire set of outcomes in terms of a space, noting that there were $\left(\begin{array}{l}10 \\ 5\end{array}\right)$ total "points in the space." He mentioned two different particular outcomes, too, suggesting he considered the set of outcomes (both the entire set and particular outcomes).

A: So it looks like what we're doing here is we have a total of - our spaces we have 5 out of the 10 questions answered, so each point in that space would represent a test where the 5 - the 5 questions that were answered were selected from the other 10 . So like if I selected, say, 1, 2, 3, 4 and 5, that would be 1 point. And then 1, 7, 8, 2 and 3, that would be another point... So we have 10 distinct questions, 5 selections. So we have 10 to the - or 10 choose 5 different points in this space.

Next, Aiden used set-theoretic language to describe to solve the problem. Note, his set theory language in the excerpt below refers not to the set of outcomes, but to the set of objects from which he was choosing (the test questions). He let $F$ represent a subset of questions (the first five questions), and he wanted to consider choosing question $q$ from set $F$. 
A: Now, how many of those points have the property that at least 2 of the first 5 were selected? So at least 2. So greater than or equal to 2 of the first questions. So at least 2 of the first 5. So the first 5, I'm just going to make a subset of these questions called F. So I'm going to call that set of questions F.

E: Okay.

A: So the number of questions that are from $F$, so $q$ is a question; $F$ is a set. And the number - the number of such questions would be greater or equal to 2. So I'm going to take all the - all the different ways that we can choose the 5 questions, so like our big space, our universe and subtract the ones that are strictly less than 2 , because that will be fewer cases to compute. So we'll take a total and then subtract away the number of questions - the number of tests who have strictly less than 2.

So the cases we are considering are where there are zero questions selected from the first and where there are 1 question selected from the first. So let's take the case first where we have zero questions selected from $F$. So that means there are 5 left over. So 5 choose 5 , there's only one way that that could - because, yeah, because we have to answer - you must answer 5, so if none of them are from $\mathrm{F}$, then there's only 5 left over. There's only one way that that could be. So we have one point, I guess. And then so if 1 is selected from the first - or 1 is selected from $\mathrm{F}$, and then the remaining 4 are selected from outside of $\mathrm{F}$, then there are 5 different ways that I could - I could choose it from an F. And then 5 choose 4 ways where I can select the remaining 4. So I select one of these, and that choice is independent of the selection out here. Because these are disjoint sets. So 5 times 5 choose 4, that's going to work out to be - so our total is 10 choose 5 , that's how big we're - our space is.

In the excerpt above we see, too, that Aiden framed the problem utilizing a total minus bad approach, that is, he decided to solve the problem by counting the total number of questions and subtracting the number of tests who "have strictly less than 2." He went on to explain the details of this computation, and at the end of his work shown below he had arrived at the answer $\left(\begin{array}{l}10 \\ 5\end{array}\right)-25-1=226$. His language suggests he used set-oriented thinking (particularly his decision to utilize total-minus-bad) in order to make a decision 
to organize his work. Specifically, he decided to subtract the bad cases "because that will be fewer cases to compute." His work above indicates that he drew upon the relationship between counting processes and sets of outcomes.

4.2.4.3.2.2 - Aiden misunderstands the alternative expression. When we revisited the problem later in the interview, I gave Aiden the alternative answer, Expression W. He at first thought that the given expression was answering the question of answering exactly two of the first five questions, and he felt that, in order to be correct, he would have to add other cases. In his work below, he wrote

$$
\left(\begin{array}{l}
5 \\
2
\end{array}\right)\left(\begin{array}{l}
8 \\
3
\end{array}\right)+\left(\begin{array}{l}
5 \\
3
\end{array}\right)\left(\begin{array}{l}
8 \\
2
\end{array}\right)+\left(\begin{array}{l}
5 \\
4
\end{array}\right)\left(\begin{array}{l}
8 \\
1
\end{array}\right)+\left(\begin{array}{l}
5 \\
5
\end{array}\right)\left(\begin{array}{l}
8 \\
0
\end{array}\right) .
$$

I then asked for clarity about what he had done in his problem, and in the excerpt he gave an explanation of how his solution was "the complement problem."

A: And 8 choose 3 ways to choose from the remaining, but what this presupposes is that you're choosing exactly 2 from the first 5 . So there should be extra terms out here to add to the cases where there are more than 2 selected from the first.

E: So you feel like that's - you're missing some things.

A: - yeah. So 5 choose 2 times 8 choose 3 plus all the remaining cases, which would be 5 choose 3 times 8 choose 2 -- 5 choose 4 times 8 choose 1 , and then 5 choose 5,8 choose zero... That would be the cases where - so this final case would be the one where you select all 5 from F, zero from the other. There's only one way you can do that. And, yeah, so I think - I think this is wrong, because it misses off these terms right here.

E: Okay. And then for yours - you subtracted off -

A: Yeah, so what I did is I did the complement problem. This kind of looks like the first one with the password where it's all - all the cases 3 or greater were considered. 
A number of other students had previously misread the alternative answer to the Test Questions problem, thinking that it had said $\left(\begin{array}{l}5 \\ 2\end{array}\right) \cdot\left(\begin{array}{l}5 \\ 3\end{array}\right)$ and thought that it only dealt with exactly 2 of the first 5 problems. Thus, in order to make sure he saw what the expression actually said, I pointed out that it was actually $\left(\begin{array}{l}5 \\ 2\end{array}\right) \cdot\left(\begin{array}{l}8 \\ 3\end{array}\right)$. He realized that he had been mistaken, and he went about calculating the numerical answers. It is noteworthy here that, unlike many other students, Aiden did not take time here to make sense of the alternative expression. He did not examine it and try to see what someone might have been thinking in order to arrive at that answer. Instead, he immediately entered the expression into the calculator to compare it to the answer he had gotten previously. He would later go on to describe what the expression was doing, but he went to a numerical check first.

\subsection{3 - Aiden identifies a particular element of the set of outcomes that is overcounted}

by Expression W. Aiden found that his answer (equivalent to Expression R) was 226, while the alternative, Expression W, was 560.

E: So what's going on with that [Expression W]?

A: Okay. What I'm worried about is I can kind of look at it [Expression W], and I can almost see how it would work.

E: Yeah, yeah, totally.

A: I have to think about this some more.

E: So again I might - so what - yeah, is there something that got counted more than once by that - 
A: Okay. Yeah. Because this is higher, plus it's kind of like the previous one. Well, all right, so suppose I choose 2 of these. 6 choose -5 choose 2 different ways that I can do that. If I selected - uh, if I were to choose them all from F - okay, so if I were to choose my - let's say I choose the first 2 , then I can choose - there are 8 left, and if I do 8 choose 3 - so that includes the case where all of them are selected from F. Okay? So how can I get that case elsewhere? Or select two this way, that would be a distinct case, and this would be a different set of remaining and then I could also - it would also include the case where I selected 4, 5, and then say, 1, 2, 3 as opposed to 1,2 and then $3,4,5$.

For the sake of time, I told him that his answer was actually correct, so he set about figuring out why the alternative answer was wrong. He said that he could see how the incorrect expression could work, and he wanted some time to think about it more. We see above that he was able to make sense of the alternative answer. I prompted him by asking if there was something that was counted more than once by the incorrect expression, and he noted that the situation reminded him of the "previous one," which was the Passwords problem. We see that he was able to detect the overcount, by identifying a particular set of questions $\{1,2,3,4,5\}$ that was counted more than once by Expression W.

He then restated his argument above, and his language suggests a coordination of a process and the outcomes it generates. He identified a particular set of test questions that was counted more than once, and that told him how overcounting had emerged.

A: So making - making this kind of explicit, if I selected 2 from $F$ and then there from what remains - there are cases where I say I selected 4 and 5 from that one subset of F, that's of size 2, and then I selected from the remaining 8, which is 6 through 9 and then 1 through 3,1,2 and 3 is a subset of F, so that's one of these from the first $5 \ldots$ And that is also counted along with the case where I selected, say, 1, 2 and selected from the other - the other remaining 8, which is now 4 through 9 - or 3 through 9 . That would be 1, 2, 3 and 4, 5. So I think that's - that will be an example of where you get double counting. 
4.2.4.3.3 - Summary. Aiden's work is an example in which a student utilized the totalminus-bad strategy on this problem. He articulated the total space of outcomes and listed some particular outcomes, indicating that he considered the set of outcomes. He subtracted off the cases in which 0 or 1 of the first 5 problems were chosen, which gave him the correct answer. When he was given the incorrect alternative answer, he initially misread it. After some time he was able to see why it was incorrect, and he did so by identifying a particular set of test questions that was counted more than once by the incorrect Expression W. He indicated that this was enough to convince him why the answer overcounted. This is another example in which an appeal to a particular outcome helped a student explain a discrepancy among two different counting processes.

\subsubsection{4-Other episodes}

4.2.4.4.1 - Casey writes out a partial systematic list of elements in an attempt to identify a discrepancy. Casey had initially gotten an incorrect answer, $\left(\begin{array}{l}5 \\ 2\end{array}\right) \cdot\left(\begin{array}{l}8 \\ 3\end{array}\right)+\left(\begin{array}{l}5 \\ 3\end{array}\right) \cdot\left(\begin{array}{l}7 \\ 2\end{array}\right)+\left(\begin{array}{l}5 \\ 4\end{array}\right) \cdot\left(\begin{array}{l}6 \\ 1\end{array}\right)+\left(\begin{array}{l}5 \\ 5\end{array}\right) \cdot\left(\begin{array}{l}5 \\ 0\end{array}\right)$. When we revisited the problem I had given him Expression R, and he was trying to understand whether his answer or Expression $\mathrm{R}$ might be correct. However, he was having a hard time seeing what might be wrong with his answer, and in fact he believed that his answer was right. He was aware of the fact that both expressions involved a case breakdown, and he felt that in both instances the cases were meant to be the number of ways of answering 2, 3, 4, and 5 of the first 5 questions, respectively. I asked him what kinds of strategies he could implement in order 
to understand the discrepancy between the expressions (and to see which was correct), and he made the following suggestion. Casey noticed that he could focus on and compare just one of the cases in the respective expressions. The last case in each expression is identical (there is one way to answer all of the first five problems), but Casey observed that he could compare how each expression handled the penultimate case, in which four of the first five problems were answered. His expression had $\left(\begin{array}{l}5 \\ 4\end{array}\right) \cdot\left(\begin{array}{l}6 \\ 1\end{array}\right)=30$ for that case, while Expression $\mathrm{R}$ had $\left(\begin{array}{l}5 \\ 4\end{array}\right) \cdot\left(\begin{array}{l}5 \\ 1\end{array}\right)=25$, and these numbers seemed manageable to him. Given the difference between Expression R,

$$
\left(\begin{array}{l}
5 \\
2
\end{array}\right) \cdot\left(\begin{array}{l}
5 \\
3
\end{array}\right)+\left(\begin{array}{l}
5 \\
3
\end{array}\right) \cdot\left(\begin{array}{l}
5 \\
2
\end{array}\right)+\left(\begin{array}{l}
5 \\
4
\end{array}\right) \cdot\left(\begin{array}{l}
5 \\
1
\end{array}\right)+\left(\begin{array}{l}
5 \\
5
\end{array}\right) \cdot\left(\begin{array}{l}
5 \\
0
\end{array}\right)
$$

and his answer, his proposed strategy is quite smart. His expression gave a total of 801, while Expression R gave a total of 226 , and trying to consider particular elements of that magnitude seemed overwhelming to Casey. Finding out how an answer of 30 and an answer of 25 were generated, however, seemed more tractable for him.

C: Well the first thing I would do is, um, just list all the possibilities for, say, choosing 4 from the first 5 and then one more question to answer. And see, because we - this solution and this solution have two different answers, so it's either 25 or $30 \ldots$ So if I enumerate them all, assuming I can do that, uh, count them up, if I find that there are 30, then I'm going to go with this one, if I find that there are 25, then I think - then I would have set up the wrong, and I'm not thinking about it the right way. And I would have chosen this other answer.

E: Okay, so there is, there does exist some means of checking, where you could enumerate them,

C: Yeah. 
E: So you actually arrive at one of those two prob - answers and then...

C: Yeah, I figure if you answer - if you can figure out - if you can figure out how many ways there are to answer, uh, 4 from the first and one - plus one, um, via calculation like this and then by brute force, then that's close enough to verify the method.

He had suggested that he could list out some possibilities, in that one case, and to calculate them by brute force, and that could help him determine which was right. Because Casey explained his thinking well, I asked him if he would be willing to write out some of the cases as he suggested. He worked for several minutes, and he produced the following image (Figure 41).

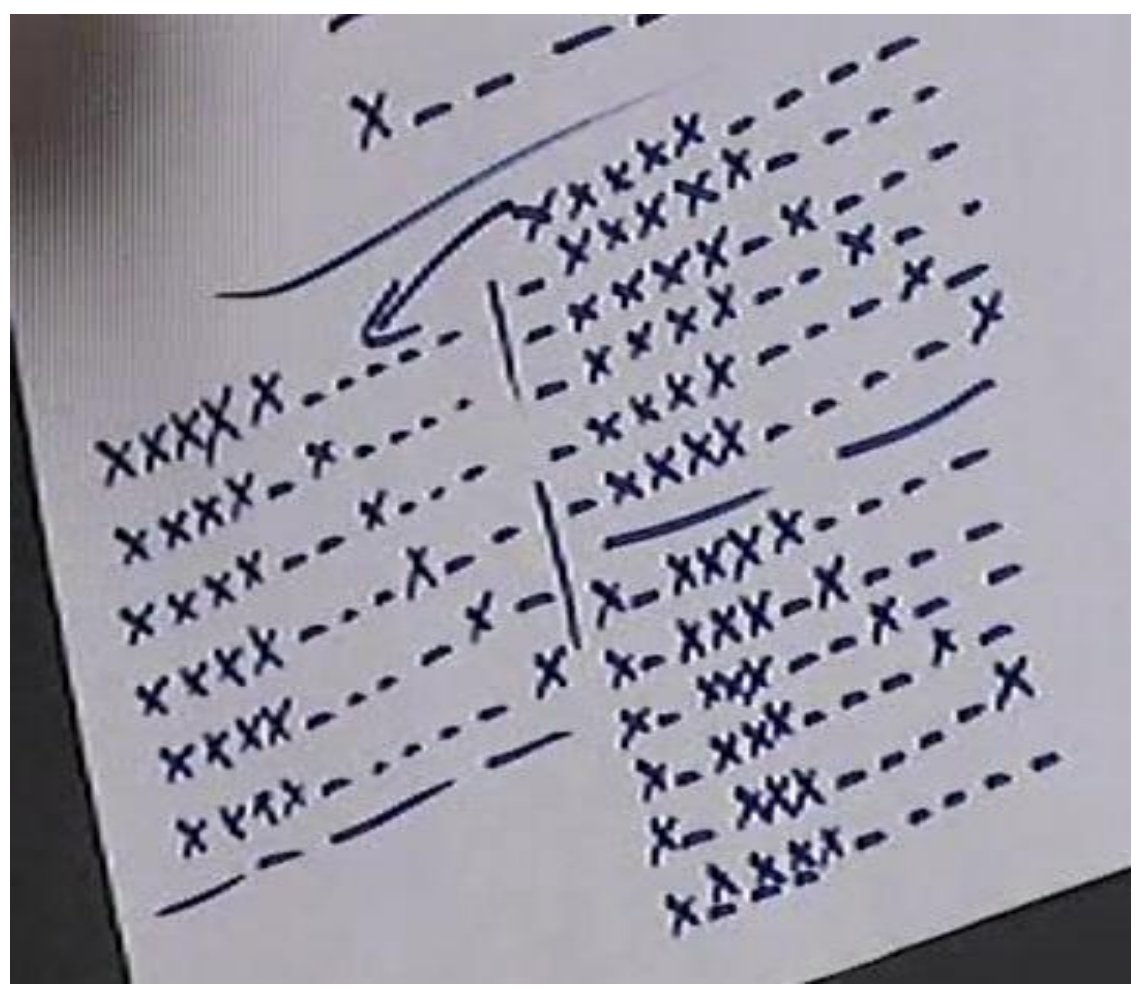

Figure 41 
Specifically, Casey started to write out his answer of $\left(\begin{array}{l}5 \\ 4\end{array}\right) \cdot\left(\begin{array}{l}6 \\ 1\end{array}\right)=30$ by writing all the examples that had 4 of the first 5 questions chosen, and then in which the final question chosen would come from any of the remaining six spots. He encoded the selection of test questions as the placing of an $x$ into one of ten spots (where each of the first through ten spots represented the first through tenth questions, respectively). On the left of the diagram below, we see that he wrote out solutions in which there were $x$ 's in the first four slots, and he arrived at 6 possibilities for where the final $x$ could be placed. Then, he wrote out the solutions in which $x$ 's were placed in the second through fifth spots, and he found 6 possibilities for where the final $x$ could be placed. He did the same, then, for when $x$ 's were placed in the first, third, fourth, and fifth spots, too. So we see that, on this page, he worked through three of the five ways to place $4 x$ 's in the first 5 spots. Already, this work was enough for him to see his error. The arrow he drew at the top of the diagram points from one solution to another, and he is indicating that, in fact, those two outcomes are actually the same. Thus, as he wrote out outcomes that his expression generated, he noticed a duplicate.

In the excerpt below, we see him expound upon this work and specifically articulate how his answer was overcounting. This exchange occurred after he had written the diagram, and he drew the arrow as he noticed two outcomes which were the same.

C: Hmm. Ahh.

E: Okay what.

C: Hang on, alright, so I've had a thought, okay. So, uh, uh, it occurs to me that my solution is counting too many, uh, just from the first 3, uh, sets or possibilities that I have here, it's, just enumerating - answering the first four and then, uh, one from 
the remaining 6... Uh, or questions 2 through 5 and then one left over, and then 1 , 345 and then 1 left over. Um, then I realized that you can answer, you know, the one before what you've already answered, or, one in the middle of what you've already answered. But in that case this - oops, is exactly the same as this [drew the arrow]... So now, and this is 6 , there's 6 here, 6 here, and 6 here, and there are going to be, and I assume that it's going to be 5, uh, different ways of, uh, ordering the first four question, it will be 6 times 5 is 30, which is to arrive at my answer, but then there are going to be too many of them because answering the first five in a row is counted, uh, too many times, 5 too many times in fact, which gets us to 25 , so there you go.

Casey then went on to explain how he had gotten repeated outcomes.

E: Okay. So the one that you drew an arrow to there, that's one, it looks like the same thing showed up in that, and also in the one on the left.

C: Exactly. And that was to, yeah, and for all of these to be correct you just have to add up all of the different possibilities, but if you're just adding all different boxes and not checking to make sure that nothing else is there you're going to get doubles, repeats.

At this point, Casey then made the following statement. I asked him how his insight about what he had just found in his example might shed light on which answer was correct, his or Expression R (I had previously asked him which he would put $\$ 500$ on, hence the mention of $\$ 500$ in the exchange below). It is interesting to see that despite the particular instance of overcounting he discovered (evidenced by the arrow), he did not feel like he understood what was going on more broadly.

C: Well I, based on this knowledge I would put my $\$ 500$ on 226 for sure... Uh, however, uh, I am, uh, mis - I'm still failing to see how the, um, how the, the, uh, choice of the way they sent up the $n$ choose $r$ calculations, uh, reflects this down here. Let me see. (Inaudible). I think I'm missing something. 
We talked about the problem a bit more, and again he indicated that he was at a loss as to what exactly was going on in the original problem. That is, he did not seem to be able to take what he had seen about a particular element being overcounted by his solution, and make sense of what that meant for the entire problem. He would have liked some further explanation and "wouldn't feel confident going in to take a test."

E: Okay, um, great. Okay any other thoughts or comments, or?

C: Well I think, if I had to do this, having done this, say this was a homework problem, and I submitted this and I got it wrong and I was told that this was the answer, and this was the math that you did it, but without understanding the concept behind why it's, why the second, why there's another 5 here in the 5 choose 1, 5 choose 2, I would not, uh, feel confident going in to take a test, for example, here, because I think I would revert to this way of looking at the problem again, yeah.

E: Okay, so you used that example to show if you had to pick that one was right you'd go with that, but you don't feel like, okay I totally understand what they were doing in that solution, and,

C: Mm-hmm, exactly yeah.

This episode with Casey provides an example of a student who cleverly investigated a numerical discrepancy in just one particular case which he felt like he could actually write out. This shows that he was able to coordinate a counting process with the outcomes it was producing, and in fact he identified a particular outcome that was counted too many times by his expression. In the model this highlights the relationship between counting processes and sets of outcomes. However, it is noteworthy that while he identified such an instance of overcounting while examining one small part of the problem, he did not feel that he really understood what was going on in the entire problem as a whole. That is, identifying the instance of overcounting did not seem to be 
sufficient to allow him to feel good about the problem and to understand what was going on more generally and why his answer resulted in an overcount.

\subsubsection{5 - Overall Summary of the Test Questions problem}

The Test Questions problem was similar to the Passwords problem in structure many peoples' strategies involved a case breakdown, and it had the "at least" constraint. In this investigation of the Test Questions problem, we saw several approaches to the problem. Joshua was able to use a correct case breakdown to arrive at the correct solution initially, though when he saw an alternative incorrect expression he could not make sense of what was wrong with it. After several prompts toward writing down particular instances of what he was trying to count, he ultimately identified a solution that was overcounted by the incorrect expression. In Kristin's case we saw the pitfalls of an incorrect model for what she was trying to count. While she initially answered the problem correctly, she was incorrect in her articulation of what constituted "same" and "different" outcomes. This ended up being very problematic for her when she had to evaluate an alternative expression. Aiden correctly utilized a total minus bad strategy, and he was able to identify a particular element that was overcounted when he compared differing solutions. Casey provided an example in which identifying a smaller part of the

problem allowed him to systematically list some particular outcomes. Doing so allowed him to detect an element that was overcounted by an incorrect expression, but he was not able to relate the instance of overcounting to the problem as a whole.

\subsection{5 - The Apples and Oranges problem}


The Apples and Oranges problem states, "How many different nonempty collections can be formed from five (identical) apples and eight (identical) oranges?" One way to arrive at a correct answer is to consider possible choices for the number of apples and oranges that can be chosen, respectively, subtracting the possibility of choosing 0 of each kind of fruit, which yields 6.9-1=53. Another correct answer involves a case breakdown ${ }^{39}$ in which one considers the number of collections of size 1 through 13. Typically the students who missed the problem attempted a case breakdown but either missed some cases or assumed that a pattern continued when, in fact, it did not. In particular, a number of students assumed that there continued to be 6 collections for every case that involved 5 or more pieces of fruit. This is incorrect, however, as restrictions on the number of oranges put a limit on the total size of the collections.

The Apples and Oranges problem is unique because it is not one that students have typically seen before. While it has a relatively simple solution $(6 \cdot 9-1=53)$, the fact that it is not immediately identifiable as a typical problem type had the effect of putting students in an unfamiliar counting situation. The Apples and Oranges problem was typically the last problem I asked students, and as a result fewer students attempted it (17 of the 22), and only 3 students revisited it. Of the initial responses, 7 got the problem right; 2 of them applied a multiplication principle and subtracted the case of no apples or oranges, yielding $6 \cdot 9-1=53$, and 5 others did some sort of numerical listing and arrived at a correct answer of 53 .

\footnotetext{
${ }^{39}$ A more detailed discussion of the cases is given in the Methodology chapter.
} 
The problem did not result in the same kinds of situations (in which students examined two seemingly similar processes) that some of the previously discussed problems had, and students tended to work on this problem a relatively short amount of time. As a result, the following discussion on the problem may feel less detailed than some of the other problems. However, I still include this problem in the results because in my analysis it received the same treatment as the rest of the problems, and because that there are some interesting situations that arose as students worked on the problem.

\subsubsection{1 - Zach's work on the Apples and Oranges problem}

4.2.5.1.1 - Overall description. Zach correctly solved the problem initially by first counting the total number of collections and then subtracting off the collection that was empty. He argued that he could chose 0 through 5 apples ( 6 total choices) and 0 through 8 apples (9 total choices). He then stated that because those amounts were independent, he could multiply and get 54 total collections of fruit, but that he subtracted one for the empty collection. While he said that he had not been thinking of a picture initially, he drew a diagram (a grid on the Cartesian plane) to represent his solution.

\subsubsection{2 - Detailed account}

4.2.5.1.2.1 - Zach uses the multiplication principle to get an initial correct answer. Zach approached the problem by considering the number of options he had for the two kinds of fruit, and because the situations were independent, he multiplied to get the total number of options. His underlined statement suggested to me that he understood that he could consider all outcomes (collections) and take care of the empty collection if he needed to. 
Z: I'm just going to start worrying about collections, and then I'll decide whether I've accidentally counted something that was empty. So, um, I think if I'm going to form a collection, that collection would have anywhere from 0 to 5 apples, and they're presumed to be identical, so, um, there are, 0 to 5 , there are 6 different, um, make sure I'm saying that correctly - right, there are the collections that consist of 0 apples or 1 apple or 2 apples, all the way up to 5 apples. Um, and that's, that's just worrying about the apples. I've got 6 different things going on there, um, and then 9 different things that can happen here, and those are totally independent notions. So, 6 different, um, amounts of apple, amounts of apple. Um, 9 different amounts of orange. Those are independent, so I would think that I could do 54 different collections.

He continued, noting that he needed to subtract the empty case, and he thus arrived at the correct answer.

Z: However, um, one of those collections that I just counted would be the option of grabbing none of the apples, um, paired with the option of grabbing none of the oranges. So I would say, since there's no other way to do an empty collection other than not grab anything, there is exactly one thing I shouldn't have just counted there if I was going to answer your question.

4.2.5.1.2.2 - Zach draws a diagram that models the problem. I then asked Zach what the 6 and the 9 represented, and he gave the following explanation.

E: Okay. And the 6 and the 9 then, what do those represent?

$\mathrm{Z}$ : Okay the 6 represents anywhere from 0 to 5 apples that I could have grabbed, so... And then the 9, 0 to 8 oranges that I could have grabbed. Um, so, I guess a typical, um, thing that I just counted here would be $\mathrm{x}$ apples and $\mathrm{y}$ oranges, and so I might have elected to grab 3 apples and 7 oranges or something like that, that's one collection, um, the largest collection being all 5 and all 8, um, and then the smallest being none of the 5 and none of the 8 . That's why I had to get rid of 1 .

Above he gave a brief mention of a particular example of what was being counted (3 apples and 7 oranges), which I take to be an instance of appealing to the set of outcomes. 
I went on to ask him if he had considered a picture; he said he had not considered one as he solved the problem, but he then volunteered the picture in Figure 42 below.

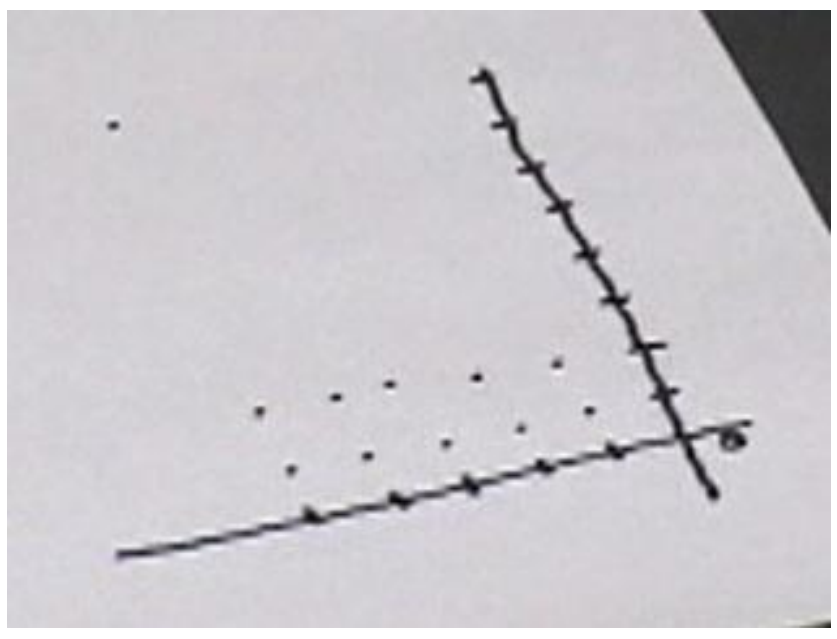

Figure 42

Z: Um, well right, I, okay, here's a picture. I could put all these dots down, um, this dot right here represents grabbing one orange and 5 apples... Um, but it seems tedious to count all these dots up, when they're just going to form a grid, and I, I know a fast way to add up a grid (laughs).

Zach noted that he could model the problem as a grid on the Cartesian plane, with each dot representing a choice of some number of apples and some number of oranges. He said that he "knows a fast way to add up a grid" (multiplication), and he thus provided some insight into a possible explanation as to why the multiplication works. It was not clear in his discussion if this Cartesian plane was how he himself envisioned the problem or whether he provided it simply as an answer to my question about the diagram. Because of the fact that he drew the diagram after he had initially arrived at his answer, and also due to the manner in which he presented the diagram, I suspect that he drew the grid more as an explanatory tool than as a reflection of his own thought process. 
4.2.5.1.3 - Summary. Zach gave a succinct answer to the problem; he recognized that the problem amounted to choosing some number each kind of fruit, he identified the correct number of options available to him, and he used the multiplication principle to easily arrive at a solution. At one point Zach referred to a particular element of the set of outcomes, suggesting he had a sense of what he was trying to count and that he utilized the relationship between counting processes and sets of outcomes. He was also able to model the problem as counting the number of integer values on the Cartesian plane, which he illustrated by drawing out the grid in Figure 42 . While the extent to which the diagram was used in his problem solving is not clear, he did use it to describe the Apples and Oranges problem in a new way.

\subsubsection{2 - Anderson's work on the Apples and Oranges problem}

4.2.5.2.1 - Overall description. Anderson began by writing out several instances of what he tried to count, and throughout his work he drew upon a visual representations he had utilized. He wrote examples down, and as he did so he thought he discerned a pattern which he used to arrive at an answer. However, in talking back through his solution with me, and in looking back at what he had written, he found an error in his initial pattern. He was able to fix it, and he gave a solid argument for why he needed to re-evaluate his original pattern. He ultimately realized what he had done wrong and found a more suitable pattern, and I interpret that it was an argument about his written representation of the outcomes that ultimately helped him to be successful on the problem.

\subsubsection{2 - Detailed account}


4.2.5.2.2.1 - Anderson writes out instances of what he is trying to count. Anderson began

his work on the problem by clarifying what was being counted, and he talked through specific collections to make sure he knew what the question was asking.

A: How many different nonempty collections can be formed from 5 identical apples and 8 identical oranges? Um, I'm a little confused at the question. Are we saying, like, are we saying a collection could be just one apple, and then another collection could be 2 apples?

E: Yup, that's right.

A: That's what I thought based on it saying non-empty. So it led me to think that.

Anderson then began to write out a visual representation for how to count the cases. I give the excerpt directly below, but then I will explain what he did as he said the following statements. I interpret that his work here can be expressed in terms of the model as highlighting the relationship between the counting process and the set of outcomes. That is, as he engaged in a counting process, he considered outcomes that were being generated.

A: Well, we have one apple, we have 2 apples, we have 3 apples, 4 apples, and 5 apples. Now we have a total of 13 fruits. How many ways can we separate if we just have one fruit? We have 2 different ways. If we have 2 fruits we can separate them as apple apple, apple orange, or orange orange, which makes 3 . If we have 3 we have apple apple apple, apple orange apple, or orange orange apple, or orange orange orange, for that matter, which makes 4. Am I missing one? No, I'm not, okay. Then for four we have, we continue this chain where we have, let's see, 4 apples, 3 apples, 2 apples, 1 apple or no apple, but 4 oranges. So there's 5 different cases for that. And then for 5 cases, or if we have 5 fruits, then we have the case where we have 5 apples, 4 apples, 3 apples, 2 apples, 1 apple and no apples, so that's 6 . For 6 , well we only have 5 apples, so the only other case would be if we add oranges to all of these. Which makes that 6 different possibilities. 
First, he noted that there were 2 options for just one fruit (an apple or an orange). Then he noted that there were three options for two fruits, and he wrote three rows:

AA

AO

OO

He then proceeded to add to this diagram for subsequent cases. For three total fruits, he added an A to the end of each existing row above, then added a row of 3 O's at the end, yielding.

AAA
AOA
OOA
OOO

And again, for four fruits he added A's to the end of each row then added a row of O's, and for five fruits he did the same thing. He ending with the following diagram, noting that there were 6 ways of choosing 5 pieces of fruit.
AAAAA
AOAAA
OOAAA
OOOAA
OOOOA
OOOOO

Then, for 6 , he argued that because he only had 5 apples to choose from, he could no longer add A's to the end of the rows. But, since he still had oranges available he could then add oranges to all of the existing rows. He thus wrote out the diagram below, which indicated to him that there were a total of 6 possible collections of size 6 .

AAAAAO 
AOAAAO

OOAAAO

OOOAAO

OOOOAO

OOOOOO

From here, Anderson argued that for any of the other cases beyond 6, there would similarly be 6 possibilities, seen below.

A: Um, it looks like it's going to continue in this fashion, so for $7,8,9,10,11,12$, and 13 we have 6 for all of them. 6 different ways, 6 different um, sets consisting of that size, and then we add them all together. That's 68 different ways, I believe. Yeah I believe it's 68 different ways.

So while his pattern had initially made sense, Anderson was incorrect that it would continue in the same way, and he thus arrived at an incorrect answer of 68.

\subsection{2 - Anderson talks through his work and uses his diagram to identify an error. I}

then asked Anderson to talk back through his work, and to explain to me what he had done. As he did so, he pointed and referred back to his diagram. As he continued to talk, he noticed an error in his work - namely that for total numbers of fruit greater than 9 , he would no longer have 6 possibilities. The underlined portion below highlights this realization.

E: And once you got past that 6 example you were able to say that all the rest of them were 6? How did you reason about that?

A: Uh, this one I felt it would just be easier if I did a - like if I didn't do a lot of calculation, instead just did, I don't know if it'd be called a brute force method, Um, but, basically I thought, okay well the set can't be of size 0 , it has to be at least size 1 , the highest size it can be is 13 because that's how many fruits we have... Um, so let's just start with 1, and then I realized, okay, it can either be an apple or it can be an orange, so we have 2 possibilities. And then, we hit two, and I said, okay well we can have 2 apples, 1 apple, or no apples, 
E: Mm-hmm

A: The remaining elements in the set are accounted for with oranges. For 3, we have 3 apples, 2 apples, 1 apple, or no apples. Um, so it continued in a fashion where it was just this size plus one. But we only have 5 apples, so we hit five apples - we hit size 5 fruits, or size 5 , and we hit $5,4,3,2,1$, and no apples, which is 6 . But when we hit 6 , it's still 6 because it'd be 5 apples and 1 orange, 4 apples and one orange, 3 apples and one orange, etc. Plus whatever oranges were already in the set to begin with... So since it can continue on in this fashion, the oranges can keep going until there's 8 of them in total, um, no wait, does that work? No that doesn't work - that's silly. It's not 6 .

E: Okay, now how come - so you're...

A: It's not 6. I'm, I must be tired or something today. Okay, it can't be 6 because if it hits size 8 , then once it hits size 8 then we have 8 oranges, let's see we have the case 8 oranges and no apples, uh, when we hit size 9 , one of them must be an apple, so we remove the case where there are no apples. So after 8 , it is no longer $\underline{6}$, it is 5 and then 4 , and then 3 , and then 2 , then 1.

I was curious about what specifically caused him to see his mistake, and so I asked him to explain what made him realize that his mistake had occurred. As he did so, he referred back to his diagram. He seemed to use the visual diagram, which included some particular elements of what he was trying to count, both as a means by which to uncover his error, and also as a means by which to explain and articulate his reasoning to me. Above, right before he realized his error, he said, "since it can continue on in this fashion, the oranges can keep going until there's 8 of them," and he wrote out several more O's in the first row. The final picture he drew and used in his reasoning is seen in Figure 43. In the excerpt below, when he said, "I'm drawing out these O's," he referred to drawing the O's on the end of the first row. 


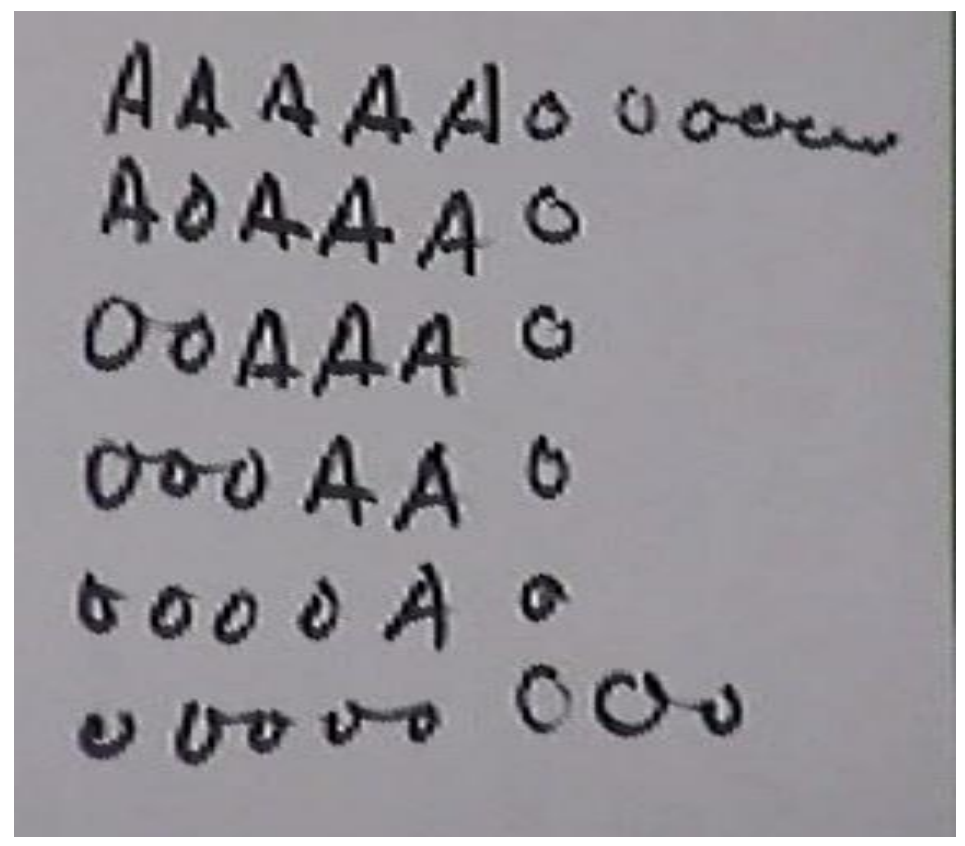

Figure 43

In the exchange below we see Anderson's reasoning about how he made a mistake.

E: You were talking through the solution, and explain what was it that caught your eye and made you realize...

A: I'm drawing out these O's, and then I realized, wait a second, if I draw out the O's on the next line, it's not going to be the same size because I already have an $\mathrm{O}$ accounted for over here. Um, I already have one.

E: Okay, say that one more time, so...

A: I wrote these lines for the case where all apples, all but one are apples, all but 2 are apples, all but 3 are apples... While maintaining the same size, which means the, um, when it's all but one are apples it means one of them are oranges, if it's all but two of them are apples it means 2 of them are oranges, but as it grows larger, um, see as this went from size 1 to size 6 it was growing larger, Because we had more apples remaining... Once it hit size 6, we don't have any more apples. Using apples as the main focus.

E: Yeah.

A: But, once we hit size 8 , if we want to grow bigger, we have no more oranges to account for, which means they need to be replaced with apples, which means this list would get smaller. 
E: Okay. And so something about drawing out all those O's, you realized, what, that that second line, there was a problem?

A: Right, that second line would have one fewer entry because the $O$ is already accounted for.

As Anderson explained his mistake, he frequently referred to the diagram in Figure 43. It is noteworthy he appealed to some specific characteristics of particular outcomes as he explained how he had detected his error. Anderson was thus able to recognize the mistake and see what needed to be fixed in order to arrive at the correct answer.

4.2.5.2.3 - Summary. In Anderson's case, we see that he began by writing out some particular examples, and he then noticed a pattern that he implemented (which was incorrect). I asked Anderson to explain his work, and as he explained his initial answer he referred back to the diagram he had drawn. In doing so, he examined particular outcomes, and he realized a mistake he had made - specifically, that the pattern he had implemented was under the assumption that he had more oranges available to him than he actually did. By looking back at what he had drawn, he saw his error and fixed his work accordingly. As we talked more, he clearly articulated exactly what had gone wrong in his work. A number of other students had also initially arrived at the same incorrect pattern that Anderson did, although they were not always successful in recognizing and explaining their error.

\subsubsection{3 - Jon's work on the Apples and Oranges problem}

4.2.5.3.1 - Overall description. Jon initially wrote out some instances of what he was trying to count, but his articulation of outcomes was overshadowed by an emphasis on 
trying to detect and apply a pattern or formula. Indeed, while he did draw upon the set of outcomes to a small degree, he seemed primarily interested in establishing a pattern. He was very focused on pattern detection, and he never took the time to evaluate carefully whether his pattern made sense. I have chosen to highlight Jon's work here in order to show an instance in which a student pursued a pattern and did not carefully consider whether the pattern would hold. In contrast to Anderson's work above (who looked to the particular instances he had written out in order to detect an error), Jon did not notice any error in his pattern. In terms of the model, I interpret that John did attempt to articulate the relationship between his counting process and the set of outcomes.

\subsubsection{2 - Detailed account}

4.2.5.3.2.1 - John discusses the issue of order. Jon began by discussing the issue of order in the problem, and he explicitly asked me if order mattered.

J: Different nonempty collections can be formed from five identical apples and how many different non-empty collections can be formed from five identical apples and 8 identical oranges, so we're going to have 5 apples and 8 oranges, and we're going to just kind of jumble them all together, so, if they're all five identical apples, and 8 identical oranges, does order matter in this case, because I'm not sure if it matters or not, I don't,

E: Okay, so what, first of all what do you mean by does order matter?

J: Uh, does, like, let's say if we have, so I know they're all identical, but let's say we have apple, orange, apple, would that be the same as saying orange, apple, apple, that's what I'm thinking.

E: Yeah, and so I think it is, because you're dealing with collections.

J: Collections? Okay.

E: Yup, yup so that would be, um,

J: So order does matter?

E: Or no, so order doesn't matter.

J: Order doesn't matter, okay, 
E: Because those, yeah, those wouldn't be counted as different.

I asked him what he meant by order mattering, and, as we see above, he referred to a particular example. I told him that he would want those to be considered the same, and so order does not matter (I should note that by this point in the interview, he had struggled quite a bit, and I thus felt okay telling him that order did not matter). In the exchange with him, it was not entirely clear to me that he felt sure whether or not order mattered. The fact that he brought up a particular example (apple, apple, orange) suggests he considered what he was trying to count.

\subsection{2 - Jon considers alternative strategies to solve the problem and seeks a pattern.}

After we clarified the issue of order, Jon thought about what strategies he might employ in order to solve this problem. He considered perhaps drawing a diagram "like the one in the previous one," (he had done the Test Questions problem previously, and he had drawn a diagram reminiscent of a "stars and bars" problem), but he did not follow up with that. He spent some more time considering what he was trying to count, and eventually he had established that he needed to consider collections of varying size. Jon then proceeded to write out some particular instances of the set of outcomes, with the goal of finding a pattern. He started writing out sets involving 1, 2, or 3 elements (the diagram is Figure 44 below).

J: Alright. So, from there, this probably wouldn't, this type of arrangement probably wouldn't work, so, I guess we could count how many, maybe the first thing we could do is start, uh, with just drawing a first thing with sets, maybe the first one we could be, one set of apples, a set of one, and set of oranges, that's just the base case,

E: Okay. 
J: Type, we could have one, and then we could have another one where we would have something along the lines of, maybe 2, so there'd be apple-apple, and then orange-orange, and we can have a total of 13 , so, and then apple and orange, and that would probably be, okay that's another, that's another, uh, form, so, oh I'm thinking maybe this is a power of 2 types of thing now, maybe. Set of 2, that's 3 sets of 2, and now say if we have a set of 3, there would be apple-apple-apple, orange-orange-orange, apple-apple-orange, and then, orange-apple-apple, and then apple-orange-orange, and that's kind of the patterns I'm starting to see when I look at this now.

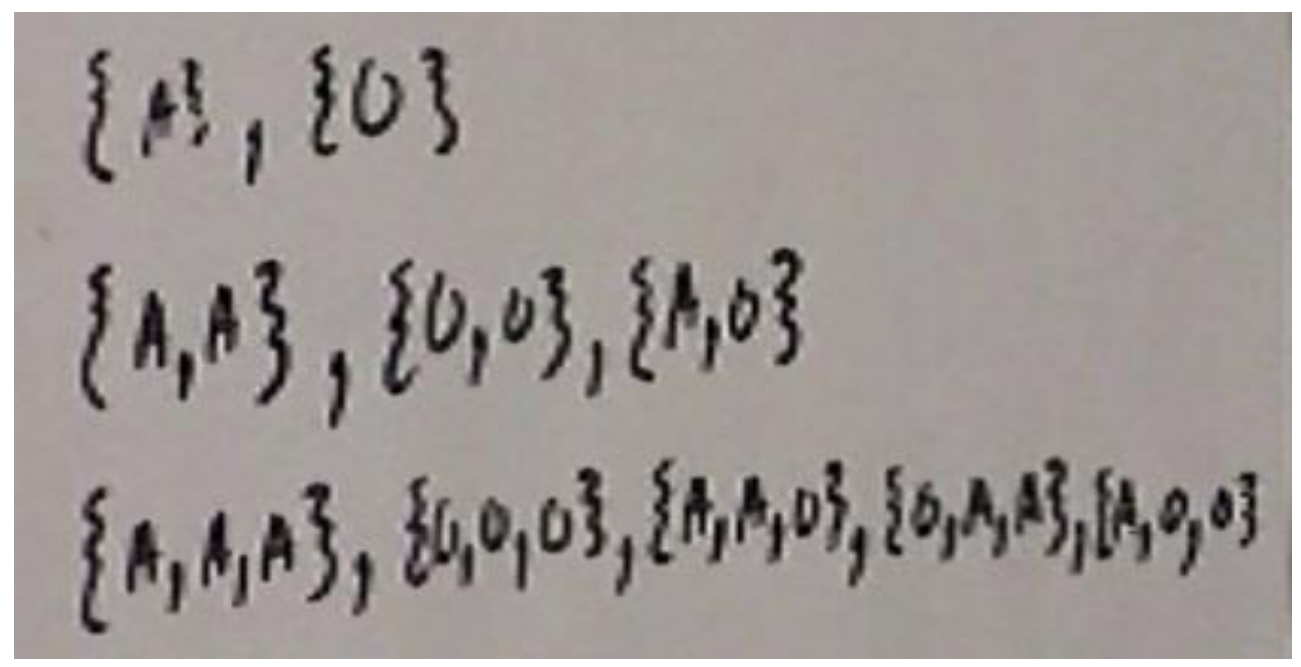

Figure 44

While Jon's activity of writing out examples did indicate that he was considering particular instances of what he was trying to count, there were a couple of problems with this approach for him. First, his language (such as "base case" and talking about a "power of two") seemed to suggest that he was looking for patterns and was not paying specific attention to the actual outcomes. That is, he did not seem to utilize the nature of the elements involved in order to make sense of how his pattern may or may not be correct. Second, Jon made some errors in his listing. As we saw in Figure 44, the third row 
contained both an $\{\mathrm{A}, \mathrm{A}, \mathrm{O}\}$ and $\{\mathrm{O}, \mathrm{A}, \mathrm{A}\}$, which was not correct (and which was different from what he had done in the second row).

E: And, I have one question, so the apple, apple, orange, and the orange, apple, apple that you have in there,

J: Uh-huh.

$\mathrm{E}$ : Um, do you want both of those in there?

J: Apple apple orange, oh yeah, no we don't, because order doesn't matter. So we can get rid of that, so we have one, two, yeah, 4 arrangements...So, yeah that's kind of the way I would see it, you would just be kind of a power of 2 type thing.

I pointed out that the third row contained both of those elements, and he recognized that he would not want to include it in that case. The pattern for 1, 2, and 3 objects is thus 2 , 3, 4, and again he (somewhat carelessly) suspected that the pattern was some kind of a power of two (it is not).

After some more time spent on the problem, Jon recognized the pattern for the number of outcomes for each number of possible objects as being one more than the number of objects.

$\mathrm{J}$ : It's looking like this is going to be, 2, 3, if you do, each set has 1, and you have 2, and if you have each set of 2 you're going to have 3 , and if you have each set as 3 you're going to have 4, so, well I'm kind of seeing a pattern there now, Uh, mmm, I guess this would be like 2, uh,

E: And would you keep going or does it seem like it's too much trouble?

J: Yeah I don't think I'd keep going on this one, I just, from this point I'd probably just look at it and try to see if I can find a pattern. Maybe I'd do like maybe one more like, 4... So apple, usually 4 I've found is good. Oh wait this one's kind of in here already. No this one's not. Yeah there's no more. So it's 1, 2, 3, 4, 5, it's always, it's always, um, one, the number of sets you can have is always one more than the, uh, it seems like there's always one more number of sets as there are number of elements in the set. Is what I'm seeing... For each one, up to 13.

E: Does that makes sense, or does it, is it more that the pattern is kind of,

$\mathrm{J}$ : That's kind of what the pattern's saying to me. 
We stopped before he actually calculated it, but his pattern would have been to add the integers $2+3+4+\ldots+13+14=104$. His pattern was correct up through 6 objects, but after that he would have run out of apples, and after 9 objects he would have run out of oranges. His language above is interesting, giving some insight into what he believes about determining mathematical patterns.

4.2.5.3.3 - Summary. Jon's work on the problem provides an instance in which a student did write down some particular elements of what he was trying to count. He was writing a few initial examples with the intent of developing a pattern - he referred to one of the examples as a "base case." However, he did not pay attention to the nature of the elements he was writing down. Instead he seemed completely focused on finding the pattern. And while he did eventually find a pattern that he thought worked, he assumed that it would continue to work for his remaining cases. Thus, we see that writing out some particular solutions did not necessarily mean that he thought carefully about the set of outcomes. In particular, he did not seem to consider the fact that he might run out of apples or oranges eventually. His focus on a pattern kept him from solving the problem correctly.

\subsubsection{4 - Other episodes}

In addition to the episodes described above, there are two other noteworthy incidents to highlight. First, there were some students who solved the problem by correctly enumerating all 13 cases and counting the number of outcomes in each case. Brandon's work below is an example of a correct execution of this solution. Second, I will show an 
example of Nancy's work, in which she was given an alternative expression, but in which she did attempt to conjecture what an appropriate counting process that yielded such an expression might have been. As was the case on the previous problems, in these two episodes I do not give the full details of the students' work on the problems, but I highlight only the aspects that are relevant to the discussion at hand.

4.2.5.4.1 - Brandon efficiently solves the problem using cases. Brandon began to list the cases systematically according to how many pieces of fruit he could choose. Figure 45 shows his work on the problem, and we see that he has listed out 13 cases.

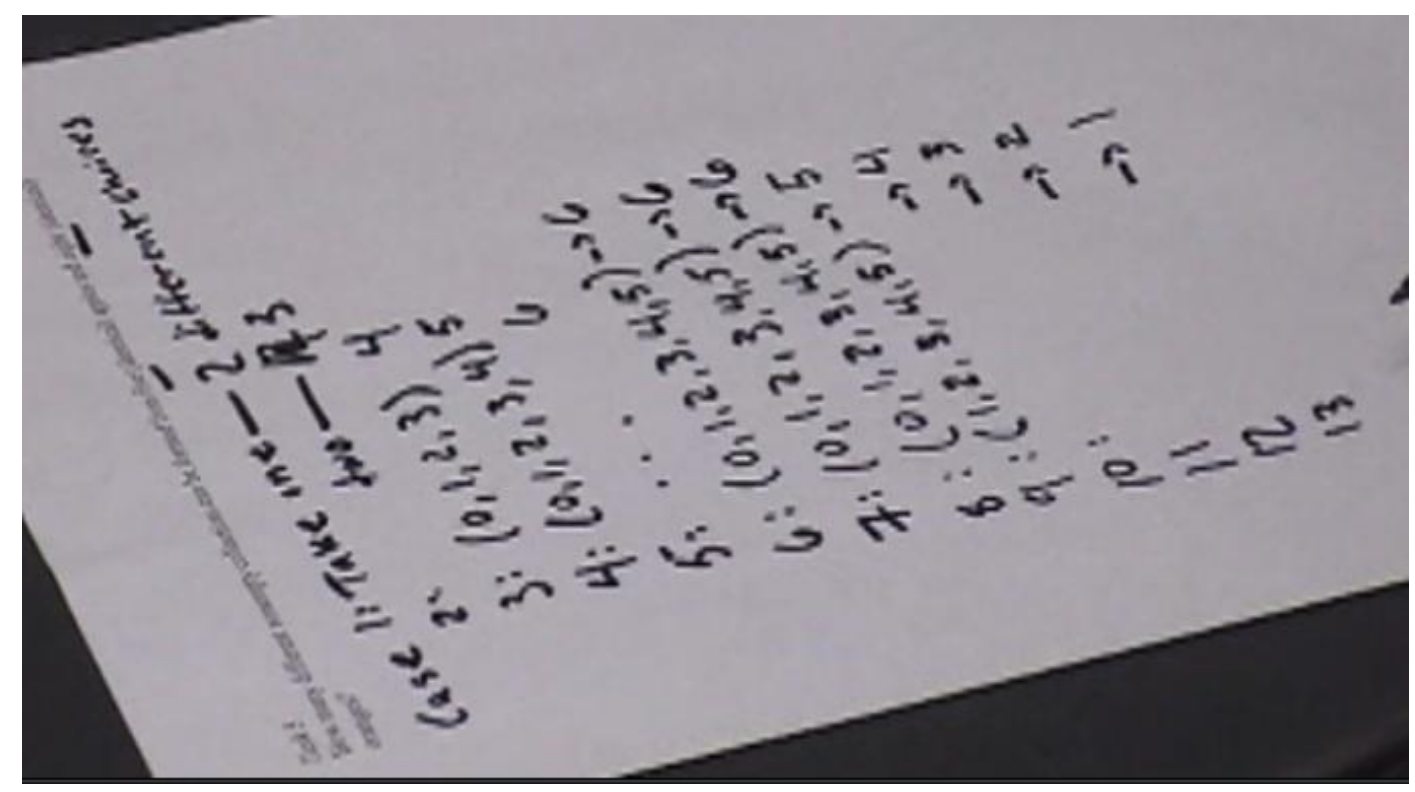

Figure 45

Unlike Jon, Brandon did not seem to be overwhelmed by the prospect of listing so many cases, nor did he try to skip steps by assuming all of the cases past 5 were all of size 6 as Anderson had. He simply listed out some of the initial possibilities and efficiently argued for the appropriate number of outcomes for each case. While he did shortcut some of the 
steps (as seen in his picture), he was quite careful to list the particular outcomes he judged to be problematic. Brandon thus arrived at an answer of $2+3+4+5+6+6+6+6+5+4+3+2+1=53$. Four other students arrived at the correct answer by listing out options as Brandon had.

\subsubsection{2 - Nancy fails to relate an alternative expression to a counting process}

Nancy had worked on the Apples and Oranges problem and had eventually counted up cases correctly to arrive at the answer of 53. In the excerpt below, as we revisited the problem I had given her a correct alternative answer 6.9-1. As mentioned above, one can see that this solution makes sense by connecting it to an enumeration process in which one could choose $0-5$ apples and then 0-8 oranges, and then exclude the possibility of choosing 0 of each.

$\mathrm{N}$ : 6 times 9 minus 1. Yeah I don't know what that is. Does mine turn out to 53, I don't know.

E: I think yours would turn out to 53 .

$\mathrm{N}$ : Why do that? Yeah I don't know where this is coming from. I have - I don't know how they did that.

E: Okay. What if I asked you to just take another minute or two and think about it...

$\mathrm{N}$ : Okay. Well it's, it's 5 plus 1 times 8 plus 1 minus 1 . So I don't know if that works all the time or not.

E: $\quad$ Okay so where could the 5 plus 1 times 8 plus 1 come from?

$\mathrm{N}$ : Well 5 apples and 8 oranges, so if that's a formula that works,

E: Okay, and how about, why does that make sense in terms of the problem. Like, what - why would 5, like why multiplication...

$\mathrm{N}$ : I don't know, I don't know.

In this example Nancy could not see why 6.9-1 could be a correct answer. Even more, there is nothing in her language above that suggests that she tried to connect the 
expression to some underlying counting process. I suspect that the new expression was so different from what she had done that she perhaps did not even know where to begin, but I still find it noteworthy that there was no attempt to try to determine the reasonability of the formula by connecting it to a counting process. This example was quite exceptional; usually students attempted to make sense of the alternative expression given to them. This example is particularly noteworthy in terms of the model. It highlights an instance in which a student was not able to examine an expression and come up with a counting process related to that expression - thus showing how the relationship between formulas/expressions and counting processes can "break." It is not just that Nancy came up with an incorrect process; she did not make an attempt to come up with a counting process at all.

\subsubsection{5 - Summary of Apples and Oranges Problem}

The Apples and Oranges problem is unique because it is not one that students have typically seen before; students had a variety of responses and approaches to the problem. Zach solved the problem easily, and there were times in his work when he referred to particular outcomes. Additionally, he was able to provide a model for how one could think about the problem using integer coordinates of the Cartesian plane. In Anderson's work we saw him utilize his drawing in order to catch an error in his initial solution and to eventually arrive at the correct answer. Jon also started writing out some cases, but he did not want to write out very many, and he applied a formula based on the emerging pattern. Unlike Anderson, though, he was not able to identify the problem with his 
solution, and he thus arrived at an incorrect answer. We concluded with a discussion of two other students - one who correctly used a case breakdown and one who did not relate an alternative expression to a counting process.

\section{3 - Summary of the Results Chapter}

In this Results chapter, I have first of all elaborated a model for students' counting activity. This model emerged as a result of my study; it is a conceptual analysis that I used as a lens through which to consider students' work on counting problems, and it was refined through the data analysis process. I described the model in Section 4.1, and I will use the model to frame my findings in the Conclusions chapter. In Section 4.2, I gave a sense of what happened during the interviews. I provided a plentiful amount of raw data from the actual transcripts, and I attempted to set these up and discuss them with a suitable amount of detail. In addition to getting a sense of how the interviews were conducted, the goal was for the reader could gain some insight into the subtleties of the tasks and the variety of approaches that students took on each problem. It is from these results that I will draw my conclusions. 


\section{Chapter 5 - Conclusions}

In this final chapter, I highlight the major conclusions I have drawn from this dissertation study. In particular, I use the model described in Section 4.1 to explicate my findings - more specific conclusions regarding students' counting activity as it relates to coordination of sets, processes, and formulas. This involves examining ways in which set-oriented thinking arose for students throughout their combinatorial problem solving. I conclude the chapter with ideas for further avenues of research and wrap up with a final summary.

5.1 - A review of the model for the coordination of formulas, counting processes, and sets of outcomes

5.2 - Student work that did not indicate set-oriented thinking

5.3 - Observed ways in which students use set-oriented thinking

5.4 - Avenues for further study

5.4 - Summary

\section{1 - A review of the model for the coordination of formulas, counting processes, and sets of outcomes}

As a reminder of the model presented in Section 4.1, which serves as a conceptual analysis of student counting activity, I briefly recall the elements of the model. Throughout the remainder of this chapter, I will relate the findings to this model. The model (Figure 46) addresses the coordination of sets and processes in combinatorial 
problem solving, highlighting relationships between counting formulas/expressions, counting processes, and sets of outcomes.

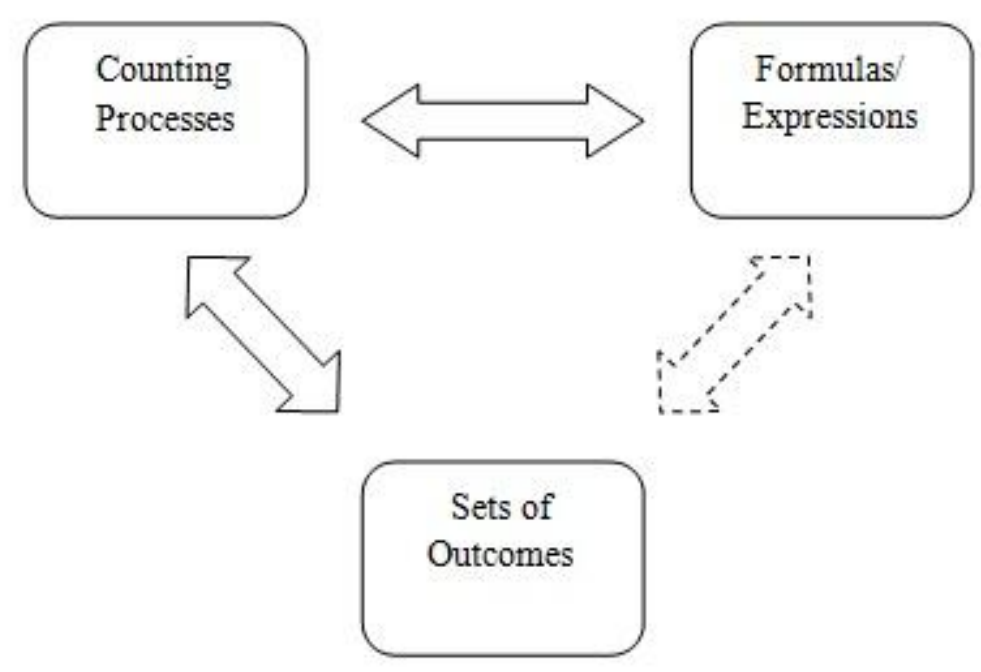

Figure 46

By Formulas/Expressions I mean mathematical expressions that yield some numerical value. By Counting Processes, I mean the enumeration processes in which a counter engages as they solve a counting problem. By Sets of Outcomes I mean those sets of elements that one can imagine being generated or enumerated by a counting process. For a given counting problem, a student may work with one or more of these components and may explicitly or implicitly coordinate them. While all of these relationships are potentially interesting in their own right, in my study I am most focused on the relationship between counting processes and sets of outcomes. It is in this relationship which students' uses of set-oriented thinking lie, and in this chapter I will answer the research question by elaborating particular ways in which students drew upon set- 
oriented thinking. As mentioned in Section 4.1.1.2, the relationship between counting processes and formulas also arose in my study, but it essentially occurred in the background; indeed, the fact that an expression could be seen as having a counting process that underlies it was something of an assumption for the students in my study. As we will see, I will use that relationship to represent student activity that did not clearly suggest set-oriented thinking. The relationship between sets of outcomes and formulas/expressions was discussed briefly in Section 4.1.1.3, but is not relevant to the findings in this chapter.

To remind the reader, I recall my research question: To what extent is set-oriented thinking relevant as post-secondary students solve counting problems? Specifically, in what ways do students use set-oriented thinking as they solve and evaluate counting problems that are susceptible to errors of over-counting? To answer the question, I organize my findings into two types. First, I show that there is evidence that students did not, on the whole, utilize set-oriented thinking (certainly not as often as they could have). That is, I have evidence of students' counting activity that indicated they did not draw upon the set of outcomes and thus did not use set-oriented thinking. There were, however, instances in which students did seem to draw upon the set of outcomes in meaningful ways. Thus, second, I describe ways in which I did observe students utilizing set-oriented thinking. I specify four ways in which students were seen appealing to sets of outcomes in their work. I will couch these findings in terms of the model presented in Section 4.1. I begin by describing some instances in which students emphasized the 
relationship between counting processes and formulas/expression, but did not clearly relate the counting processes to sets of outcomes.

\section{2 - Student work that did not indicate set-oriented thinking}

One finding of my study is primarily a negative finding. Specifically, I was disappointed to find that when left to their own devices, students, on the whole, tended not to consider the set of outcomes as they solved counting problems. Throughout students' work on the problems, I repeatedly observed instances in which students did not give evidence of set-oriented thinking, but rather in which they appealed to counting processes, and in many cases simply applied patterns and formulas. While pattern hunting and the use of formulas was, at times, beneficial for students, in this discussion I want to emphasize some of the problems students faced when their work was not grounded in a consideration of the set of outcomes they were trying to count.

I suggest that the evidence discussed in this section can be illustrated by the diagram below (Figure 47). In particular, we see examples of students who utilized the relationship between counting processes and formulas/expressions, but who did not seem to draw upon sets of outcomes in any way. 


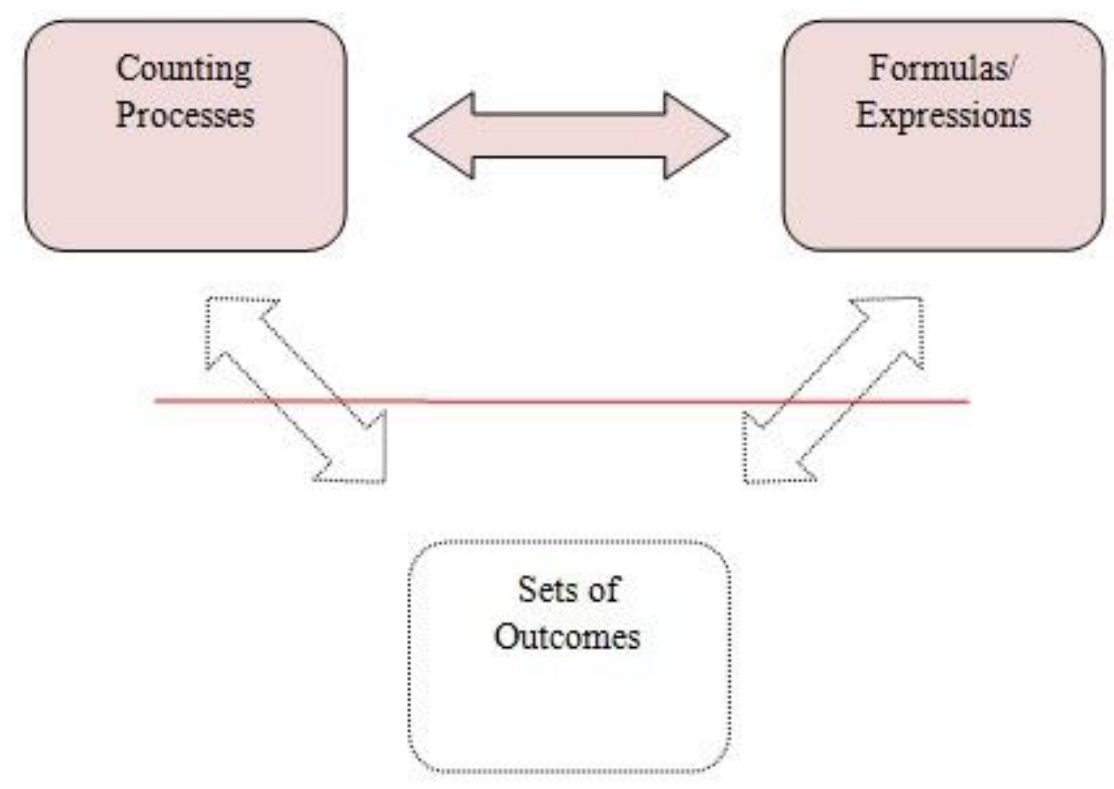

Figure 47

On many occasions, students solved problems without appealing to the set of outcomes in any way. As an example, we consider Kim's work on the Passwords problem.

K: Alright well I always start these problems ${ }^{40}$ by putting the dash things in it,

E: Okay.

K: So 1, 2, 3, 4, so we need at least 3 E's, so the option of having exactly 3 E's, so the rest of them will be open, 26 options for all the letters in the other 5 slots, and it's an and, so you multiply, and then I'm thinking that you have 3 E's are there are 3 spots out of the 8 areas where they could fall into.

${ }^{40}$ Kim did not give further indication of what she meant by "these problems." 
In her solution, she wrote out 5 dashes, wrote 26's in each of the dashes, and then wrote $\left(\begin{array}{l}8 \\ 3\end{array}\right)$. Her final answer was $26^{5} \cdot\left(\begin{array}{l}8 \\ 3\end{array}\right)$, as she explains above. She did not make any other marks on the page, and she did not verbally indicate that she considered the set of outcomes at all (when asked if she had pictured a particular password, she said that she had not). She thus solved the problem without giving any evidence that she had considered the set of outcomes. In this case, Kim arrived at an incorrect answer, one that is too large, and she did not seem to have a mechanism for determining that the answer was incorrect. Additionally, when she talked about "spots" above, her language of "where they fall into" indicate to me that she is thinking in terms of a counting process, perhaps dropping letters into slots. That is, while it may seem as though the slots approach indicates thinking about outcomes (because it could represent a concrete way to consider what one is trying to count), Kim's work above is an example in which the slots were not necessarily related to set-oriented thinking.

As another example, we consider Jon's work on the Passwords problem. He gave an answer of $8 \cdot\left(\begin{array}{l}26 \\ 1\end{array}\right)+\left(\begin{array}{l}8 \\ 3\end{array}\right)$, and his discussion of his solution below suggested that he made no clear attempt to relate the formula to a set of outcomes.

J: Ooh, well, I guess my first thought to do this would be first to draw out how many, uh, total letters there are, so there's 8 upper case letters, so, we could have 1, 2, 3, 4, 5, 6, 7, 8, uh, slots to fill. And since each, uh, slot can have, uh, 26 different letters, and we're looking for 3 E's, I'm guessing that we're going to do something along the lines of, have each one of these little slots be something along the lines of, uh, 26 total letters, and we're going to choose, just choose 1 of them, because we're only going for E's. And since this whole thing is going to be, there's 8 slots, 
I'm going to say that this would be, like, 8 times 26 choose 1 , but because there's, we're looking for 3 E's, we're probably going to be wanting to look for uh, this whole thing like 8 choose 3 or something like that, and that's plus 8 choose 3 , and I'm really not sure what I'm doing, but that's the best that I can go for.

While this solution had some potentially relevant components, it did not make real sense as a possible solution. I suggest that his work above indicates that Jon was not coordinating what his counting process was actually doing with the desirable set of outcomes. What he provided was, really, a guess, and he did not seem aware that considering a set of outcomes might be a useful resource for solving the problem.

Another example in which students' work reflected a lack of attention to the set of outcomes is through the application of incorrect formulas. This is a common phenomenon, and I found it borne out frequently in my data as well. As a specific example of students' incorrect application of formulas, Makaena used the wrong formula in her work on the Passwords problem. There had been a selection with repetition problem on the survey ${ }^{41}$ that the students had done prior to the interviews, and Makaena thought that the Passwords problem was another such problem. As seen in her words below, she decided to implement a formula with which she was not familiar in order to answer the Password problem.

\footnotetext{
${ }^{41}$ At Euler's Donut Shop, there are eight different kinds of donuts to choose from (maple frosted, glazed, chocolate frosted, etc.). Suppose you want to fill a box with a dozen donuts to bring back to your friends. In how many different ways could you do this? For example, you could have half glazed and half chocolate. This problem was adapted from Tucker (2002).
} 
M: Oh, so one thing, after I turned in the survey, I was talking with someone in one of my other classes and they randomly said, oh there's this formula for replacement, choosing with replacement, that I'd never known. Um, so I think it was, if you have uh, $\mathrm{n}$ things to choose from, and you wanted to pick $\mathrm{k}$ of them, it was $\mathrm{n}$ minus $\mathrm{k}$ plus 1 choose $k$, I think that worked ${ }^{42}$.

She had some idea of what she wanted, but she did not take care to ensure that the formula gave her what she actually wanted to count. Unfortunately, misapplying this formula resulted in an incorrect answer. She gave no indication that she could justify the use of the formula or that it would make sense as a process that would give her the desired set of outcomes (namely 8-letter passwords with at least 3 E's).

Another particular way in which students tended not to consider the set of outcomes was that, during their initial problem solving ${ }^{43}$, students infrequently considered particular examples of what they were trying to count. I recorded 28 different occasions in which I specifically asked students if they had considered a particular example of what they were trying to count, and 24 of those times they said that they had not. It is not clear why students did not have particular examples at the forefront of their mind (particularly given the potential usefulness of such examples), but there are a couple of episodes that arose that suggest reasons for this. First, at least two students mentioned a preference for

\footnotetext{
${ }^{42}$ The reader may note that this is the incorrect formula; she later changed it to $n+k-1$ choose $k$, which is the correct formula.

${ }^{43}$ I emphasize "their initial problem solving" because, as we will see in subsequent sections, students did, at times, consider particular elements of the set of outcomes to their benefit when they revisited their problem and looked at alternative answers. However, in their initial approach to the problem, they tended not to think of examples of what they counted.
} 
"abstract" rather than concrete thinking. In the excerpt below, Mike indicates that he was trying to think abstractly, and this was a reason for not considering particular passwords.

E: Um, did you ever consider - were you considering particular passwords at all at any point?

M: No. Hmm-mm.

E: Okay, were you envisioning anything, or were you just sort of thinking...

M: I was thinking as abstractly as possible about the passwords.

Second, some students indicated that they did not like to think about actually counting large sets, and so they did not even let themselves think of what was actually being counted. For example, we consider some of Daniel's work on the Cards problem.

E: Okay, great, and um, did you at any point, like were you picturing pairs of cards, were you, were you picturing, or was it - were you just imaging sort of drawing a first card and then drawing a second card, or just thinking in terms of numbers?

D: Um...I don't know, I didn't...like think about possible, like, actual possible pairs of cards - because, I don't know, I don't really like to count things that are more than 2 or 3.

E: Sure, yeah, okay (laughs).

Daniel said that he did not "like to count things that are more than 2 or 3 ." I can only speculate, but it seems like this phenomenon of not wanting to consider large numbers could have an effect on students' tendencies not to identify or consider particular elements of the solution set that they are trying to count.

On the whole, students tended not to think about particular elements they were counting and did not seem to recognize the potential value of considering a particular element of the set of outcomes. I cannot say for sure why they did not tend to consider such an element. It may be the case that it simply never occurred to them, or they may have thought a single outcome would have represented just one example and would not 
have given them further insight into the problem. We will see in the next section, however, that there were, in fact, instances in which students did refer to a particular element, and that sometimes this helped them significantly in their problem solving process.

In grounded theory, it is common for hypotheses to emerge out of a study, and these hypotheses could be tested in subsequent studies. The evidence of students not drawing upon the set of outcomes described in this section has led me to formulate hypotheses related to students' counting activity. One hypothesis from the data described above is that students limit themselves in their counting activity by not drawing upon sets of outcomes. More specifically, I hypothesize that while students may, at times, be able to successfully solve problems by relating counting processes to formulas/expressions, errors exist that are virtually undetectable without drawing upon sets of outcomes. Thus, I suggest that students who do not draw upon sets of outcomes are particularly susceptible to a slew of counting errors that are difficult to detect.

\section{3 - Observed ways in which students use set-oriented thinking}

While I have shown examples of ways in which students operated without considering the set of outcomes, and while I have made the case that students did not do this often, there were occasions in which students did draw upon the set of outcomes. I now highlight the ways in which they did utilize set-oriented thinking. In some ways, my whole study was designed to get at how students considered the set of outcomes, and I share my findings below. The ways in which I observed students using set-oriented 
thinking as they counted is organized into categories, which are outlined below, and I elaborate on them fully in the pages that follow. I have chosen to organize instances of set-oriented thinking according two major ways in which students drew upon sets of outcomes. First, their language and activity sometimes referred to the set of outcomes as a whole; second, their language and activity sometimes referred to a particular element of the set of outcomes. Throughout this chapter, when I talk about an instance happening a certain number of times, I mean that there were that many occurrences of a given situation throughout the interviews. One "instance" meant that I recorded a phenomenon for a given student on a given problem - that a phenomenon occurred at least once. So something occurring "25 times," for example, does not include repetition of the same activity by the same student on the same problem.

This set of categories forms an emerging framework for the role of set-oriented thinking in combinatorial problem solving and thus serves to flesh out the model (see particularly Figure 27). This discussion serves to further investigate the relationship between counting processes and sets of outcomes and should further inform what is entailed in set-oriented thinking.

\subsection{1 - Students consider a set of outcomes as a whole}

In identifying ways in which students drew upon set-theoretic principles, there were times when students' language or activity suggested that they conceived of some set of outcomes as a whole. I say as a whole in order to emphasize times in which students organized the larger set of outcomes in some way, as opposed to times in which students 
would identify a particular element of the set of outcomes, which will be discussed in Section 5.3.2.

Many times, when students referred to the set of solutions in its entirety, it helped ${ }^{44}$ them organize their work in some way. Specifically, this meant students could situate their set of outcomes within a universal space, either by partitioning their set of outcomes into subsets (with the goal of counting each particular subset), or by considering the complement of their set of outcomes (in relation to the universal space). Below I will discuss these occurrences separately - students' uses of case breakdowns, and students' uses of the "total-minus-bad" strategy. In terms of the model described above, both activities fit into the relationship between counting processes and sets of outcomes. Formally, both cases and total-minus-bad can represent a set partition, and when a student engages in a counting process that represents such a partition (even implicitly or informally), it may be possible for the student to naturally relate that process to the set of outcomes.

5.3.1.1 - Cases. A common way in which students considered the entire set of outcomes (and organized their work) is through breaking up their set of outcomes in some way. At times students used set-theoretic language (such as "partitions" or "outcomes") in order to describe their work, but more often they referred to this activity as involving "cases."

\footnotetext{
${ }^{44}$ As we will see, being able to conceive of an entire set of outcomes, and even being able to thus organize or structure that set in meaningful ways, did not always lead to overall success on the problem (students might have been able to see the structure of how to proceed - to count up a bunch of cases and add them, for example - but the students might not have been able to count each case correctly.) However, the organizational benefit of conceptualizing the set of outcomes as a whole was substantial, and it seems to make up an important aspect of set-oriented thinking.
} 
Sometimes students explicitly related their case breakdowns to a partition of the set of outcomes, while other times they did so implicitly. Other times still it was not clear whether students related their case breakdowns to the set of outcomes at all. While a student's reference to cases did not necessarily indicate set-oriented thinking, there were times in which the cases language was tied to the set of outcomes. I believe that it is these instances that particularly contribute to our understanding of set-oriented thinking ${ }^{45}$.

In this section I will discuss two kinds of examples. First I will give instances of students whose work indicated that their counting process partitioned the set of outcomes, and so their case breakdown showed an instance of set-oriented thinking. This will include both students who used explicit set-theoretic language and students whose reference to the set of outcomes was more implicit. Then, I will give an instance of a student who implemented a case breakdown without showing any evidence of considering the set of outcomes. Thus, in the next two subsections I will discuss both case breakdowns that seemed to be linked to the set of outcomes and case breakdowns that were not clearly linked to the set of outcomes.

5.3.1.1.1 - Case breakdowns linked to the set of outcomes. I first provide an example of a student who explicitly used set-theoretic language to describe a case breakdown (and a partition of the set of outcomes); we recall Casey's work on the Password's problem.

\footnotetext{
${ }^{45}$ While I do not claim that we cannot learn from students' references to "cases" that are not explicitly linked to the set of outcomes, I believe that the phrase "cases" can at times be used colloquially. For some students such as Kristin or Nick, it was a problem solving strategy that they used that was not grounded in set-oriented thinking. Thus, every time students said "cases" in my study, I did not automatically take that to signify set-oriented thinking; I tried to look for further evidence in their work and language that indicated they related their cases to the set of outcomes.
} 
E: Okay. Great, and so can you - that makes what you said. How did you know then to go to the $9 \cdot 13$ plus the $3 \cdot 12$.

C: Well I figured you could break it up into two different cases, because the - given the option of choosing a face card and then a heart, it can either be a non-heart face card and a heart or a heart face card and a heart, and you add those together and so you have a certain number of possibilities in this set of outcomes, and a certain number of possibilities in this set, and you just add them together and that would be the total number.

Casey's explicit language about organizing possibilities (outcomes) into a case breakdown reflects set-oriented thinking. He describes two different kinds of possibilities within his set of outcomes, and he recognizes that his case breakdown (he broke the problem into whether or not the first card was a heart, yielding $9 \cdot 13$ and $3 \cdot 12$ for his two cases) allowed him to count the separate kinds of possibilities and then ultimately add them together.

Daniel also used the notion of partition as he evaluated an alternative solution. In his work on the Passwords problem, he had arrived at a solution of $\left(\begin{array}{l}8 \\ 3\end{array}\right) \cdot 26^{5}$. When we revisited the problem, I gave him the correct answer

$$
\left(\begin{array}{l}
8 \\
3
\end{array}\right) \cdot 25^{5}+\left(\begin{array}{l}
8 \\
4
\end{array}\right) \cdot 25^{4}+\left(\begin{array}{l}
8 \\
5
\end{array}\right) \cdot 25^{3}+\left(\begin{array}{l}
8 \\
6
\end{array}\right) \cdot 25^{2}+\left(\begin{array}{l}
8 \\
7
\end{array}\right) \cdot 25^{1}+\left(\begin{array}{l}
8 \\
8
\end{array}\right) \cdot 25^{0}
$$

and asked him to make sense of it.

D: So, uh, obviously you can choose, uh, if you, again you know kind of partition the set of all possible combinations, um, and then out of those count how many are, um, the ones that you want, right. 
The excerpt above is noteworthy for two reasons. First, it seems that Daniel was able to think of solving the counting problem as enumerating some set of objects (he called them combinations), and that that set could be partitioned according to how many E's are in each password. Second, it seems that understanding the partition allowed him to make sense of the alternative solution he was given. This is thus an instance in which setoriented thinking appears to have helped a student make sense of an alternative solution.

While Casey and Daniel both used set-theoretic language to describe their case breakdown, they were exceptional. Typically students referred to cases but did not use explicit set-theoretic language. For instance, we see below that Joshua utilized a case breakdown on the Test Questions problem. In this episode, Joshua's work reflected cases which I took to be a partition of the set of outcomes, although he did not explicitly use such set-theoretic language such as "sets" and "partitions."

$\mathrm{J}$ : Um, then what I do, I will do is, uh, from the first group, uh, I will pick up 5, 2 questions from it, $5 \mathrm{C} 2$, the rest of the 3 questions will automatically, I need to do it from the other groups, I don't worry about that much.

E: Okay.

$\mathrm{J}$ : Uh, then, the uh, uh second question, second case I will think about this like $5 \mathrm{C} 3$, in the first group picking up 3 questions. And, uh, third one is $5 \mathrm{C} \mathrm{4}$, And fifth one is $5 \mathrm{C} 5$, all the 5 questions in the first group I'll do it.

But, the thing is, when I do the first 5 questions, $5 \mathrm{C} 2$, that means from the rest of the 5 I need to do 3 of them, which is 5 C 3 . This is my complete event.

E: Okay.

$\mathrm{J}$ : Then, from the first group, if I do the $5 \mathrm{C} 3$ questions, then from my second group I'm doing $5 \mathrm{C} 2$. Then comes $5 \mathrm{C} 4$, the second group is $5 \mathrm{C} 1,5 \mathrm{C} 5$ and the second group is, I'm not left out with anyone. 
There were also times in which students utilized case breakdowns that suggested setoriented thinking but ultimately did not arrive at the correct solution to the problem. For example, Anderson's initial answer to the Password problem was

$$
1^{3} \cdot 25^{5}+1^{4} \cdot 25^{4}+1^{5} \cdot 25^{3}+1^{6} \cdot 25^{2}+1^{7} \cdot 25^{1}+1^{8} \cdot 25^{0} .
$$

He explained that he considered counting exactly 3 E's, exactly 4 E's, all the way to counting exactly 8 E's, and thus his case breakdown was to count 8-letter passwords with exactly some number of E's, and each case represented those passwords with exactly three through eight E's, respectively. The idea underlying his work is correct, and in fact I believe it suggests set-oriented thinking; even if he did not use the language, he wanted to break the set of outcomes into subsets based on the number of E's in each password. Doing so created a partition of all the desirable 8-letter passwords containing at least 3 E's. It is a partition because the cases are disjoint, and together they cover the entire set of outcomes. Thus, Anderson's case breakdown is actually correct; his answer is wrong only because he counted the cases incorrectly.

Many of the students, like Anderson, correctly broke the problem into appropriate cases, but then got the problem wrong because they did not count the cases correctly. Overwhelmingly, students did seem to have a good sense of what their case breakdown should entail. Students attempted case breakdowns 61 times, and 57 times (93\% of the time), the case breakdowns were set up correctly - they were disjoint and complete. It was the implementation of actually counting each case that was problematic for students. 38 students got the case breakdown right but miscounted the cases, so although students 
broke the cases down correctly $93 \%$ of the time, $67 \%$ of the time the cases were not counted correctly.

The fact that students often found correct case breakdowns but still missed problems is noteworthy for a couple of reasons. First, it seems that a case breakdown is a fairly natural thing for students to do. There could be a number of factors that contribute to this. It could be because cases arise in other mathematical disciplines, or because cases are a mathematical concept to which mathematics majors are accustomed. Or, it could be because some of the problems very much lent themselves to a case breakdown (particularly the Passwords problem and the Test Questions problem). However, regardless of the reasoning, it seems that the notion of case breakdown could be one way for researchers to gain insight into set-oriented thinking. If a strategy of cases is already something students naturally consider, perhaps we could investigate how to tie their existing notions more clearly to a partitioning of the set of outcomes. That is, cases seem like a natural point of connection in students' work on counting problems as a way to reinforce set-orienting thinking. Second, this phenomenon is important because it gives some evidence that a case breakdown alone is not enough for success on a problem. Even when students could very eloquently describe a case breakdown (even in terms of a partition of the set of outcomes), they were not always able to get the problem right.

5.3.1.1.2 - Case Breakdowns not clearly linked to the set of outcomes. There were also instances in which students referred to a case breakdown but did not clearly engage in set-oriented thinking. As a contrast to the examples above, we describe Kristin's work on the Passwords problem. 
K: Okay. So I have 8 slots, and I want at least 3 E's, so I can have 3 E's, 4 E's, 5 E's, 6 E's, 7 E's, or 8 E's, so I would do it by cases.

E: Okay.

K: And then, so if I have 3 E's, I would do that first. And I have, uh, um, 3 choose 3, I think, and then I have to pick 5 more so take the E out of there, so 25 choose 5. And then the second case would be if I pick 4 E's, so 4 choose 4 , and then 25 choose 4, and I add them because, uh, they're cases,

E: Okay.

K: So they're not connected to each other, is how I think about it.

She utilized the case breakdown, and while she did not explicitly say it, it is clear that she was correctly breaking the problem into counting the number of passwords with $3,4,5$, 6, 7, and 8 E's, respectively. She volunteered an explanation for why she added, and she noted that she added because the cases were not connected to each other. It is not clear to me exactly what that meant to her; I suppose she could be trying to get at the disjointedness of the cases, although I cannot say for sure. Wanting to know more about her motivation, I went on to ask her to explain why she had done cases, and we had the following exchange.

E: Okay. Sure. Um, and can I ask what made you, what made you think to do cases?

$\mathrm{K}$ : 'Cause it says at least 3, so I know I can have up to 8 ... So, I always do cases normally when I see that I can have multiple ways of doing it.

E: Okay, great, and then you had mentioned, um, that you add them up because, because why, can you say more about why you added and...

K: I added them because, um, they aren't connected, so, because I can have this or the second case, or the third case, and when it says or I always think of add. And for and I always think multiply.

E: Okay. And so, would you say that you kind of had that discussion with yourself as you were solving that, like, or did it kind of just automatically happen?

K: Um, I used to, but now that I've done these I just think I do it automatically. So cases I always know, add them. 
I interpret that the above exchange indicates that Kristin's decision to utilize a strategy of "cases" was not related to outcomes but was based on a memorized rule that when she saw an "at least" problem she should use cases. Her decision to use a case breakdown did not seem to be related to an organization of what she was trying to count, even implicitly. Furthermore, she indicated that cases and addition were automatically associated for her. Even when asked directly, she did not justify mathematically why she broke the problem into cases, but instead she drew upon her own prior experience with counting problems and "at least" problems. She also focused on words like "or" and "and," seemingly relying on key words to make decisions about her work. Her response is fundamentally different from some of the above students like Daniel, Casey, and Joshua, who clearly seemed to show more evidence of set-oriented thinking.

This concludes the discussion of cases, and, in sum, we see that while the settheoretic "partition" language might most accurately reflect an underlying set-theoretic principle, the idea of organizing a set of outcomes can be implicitly captured in students' work. Cases provide one way in which counting processes can be tied to sets of outcomes, although there were times in which students used case breakdowns without clearly relating them to the set of outcomes.

5.3.1.2 - Total minus bad. There were also a number of instances in which students employed a strategy of "total-minus-bad" - students counted the total number of outcomes and subtracted off those outcomes that did not satisfy a particular constraint (the "bad" outcomes). In some instances, students clearly linked their work to a set of outcomes (sometimes this was prompted, sometimes not), using varying degrees of set-theoretic language. When this happened, I believe it 
reflected set-oriented thinking, providing examples of how total-minus-bad can be a way to coordinate the counting process with the set of outcomes.

In Peter's work on the Passwords problem, for example, his language (and more pointedly his drawing) indicated that he was organizing the entire set of 8-letter passwords into two subsets: those that contain two E's or less, and those that contain 3 or more E's (I had asked him if he was picturing anything, and he put forth the Venn diagram below). While he does not use set-theoretic language like "partition" or “disjoint," his drawing (Figure 48) and description do suggest an organization of the set of outcomes.

P: There's 8 slots, 26 different choices of letter for each slot, so it's 26 times 26 times 26, 8 times, it's $26^{8}$, that's the total. But that counts all the ones that have, you know, any number of E's maybe, um, so I want to subtract all the ones that will have either 0 E's or $1 \mathrm{E}$ or $2 \mathrm{E}$ 's, and so if I take all of them, and I subtract all the ones that have 0,1 or $2 \mathrm{E}$ 's, that should give me the number of all the ones that have 3 or more E's. 


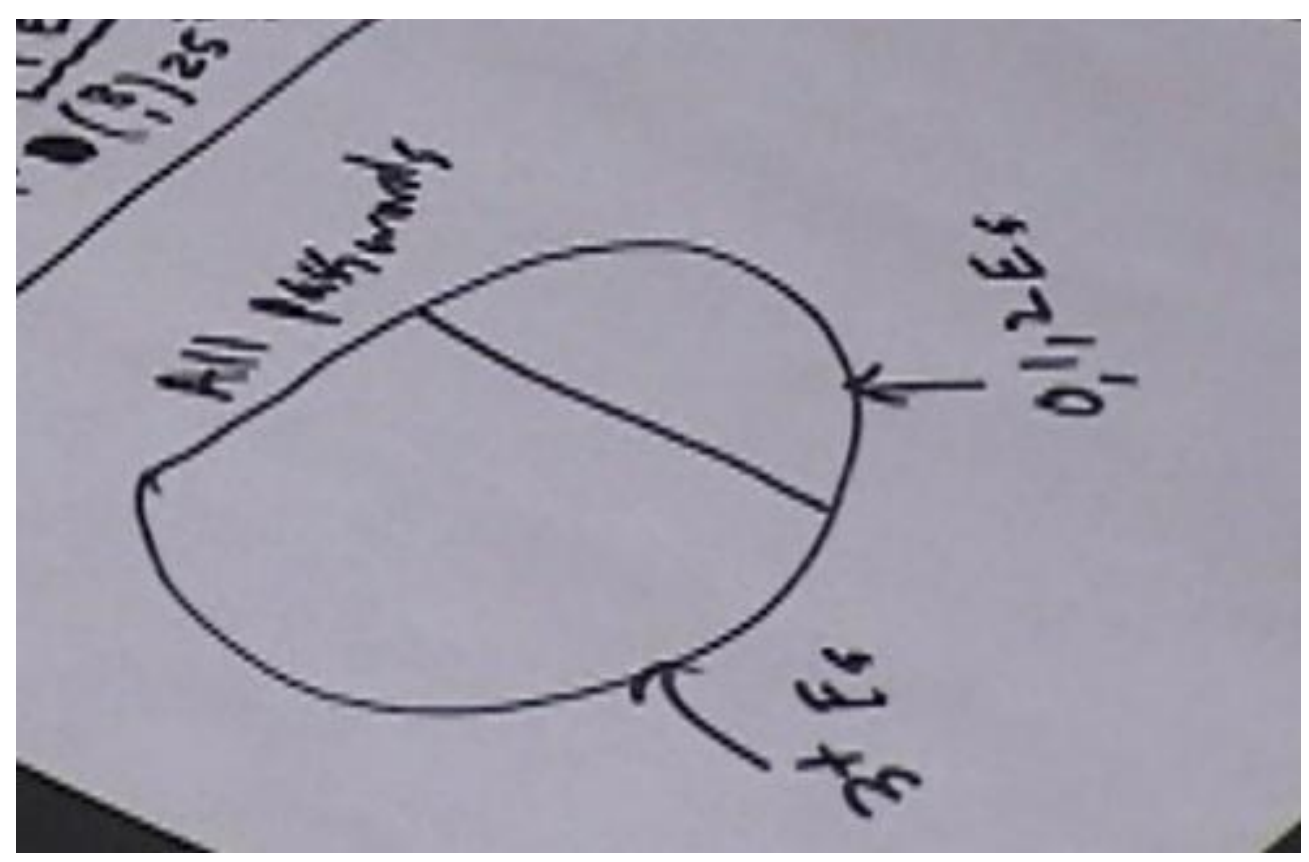

Figure 48

The Passwords problem was the problem in which the total minus bad strategy arose most frequently. Students made similar "total minus bad" comments 17 times total in the interviews, and 8 of them were on the Passwords problem. All 5 of the students who got the problem correct initially did so using this same total-minus-bad approach. One other student (Joshua) correctly counted the total but forgot to subtract off the case with zero E's, and two other students (Nick and Matthew) correctly identified the total minus bad structure but counted the cases incorrectly. The Test Questions problem was also a natural place in which students could have utilized the total-minus-bad approach, but only two students (Aiden and Paige) correctly implemented the total minus bad strategy. Three students successfully used total-minus-bad on the Apples and Oranges problem by subtracting off the case in which no piece of fruit was chosen. 
Sometimes case breakdowns and the total minus bad strategies were used in conjunction with one another. On 11 occasions students utilized a case breakdown within a total minus bad strategy - that is, they used cases when counting the "bad" outcomes. We see this, again, in Peter's work on the Passwords problem. He correctly organized the bad outcomes into cases (they were broken up into cases according to the number of E's in the password), yielding an answer of

$$
26^{8}-\left[\left(\begin{array}{l}
8 \\
2
\end{array}\right) \cdot 25^{6}+\left(\begin{array}{l}
8 \\
1
\end{array}\right) \cdot 25^{7}+\left(\begin{array}{l}
8 \\
0
\end{array}\right) \cdot 25^{8}\right] .
$$

While there were a number of factors that led to my choice of problems, I did not choose problems based on whether the total-minus-bad strategy would be a viable option. It turns out that the Passwords problem and the Test Questions problem could fairly naturally be done using total-minus-bad, while the Groups of Students problem did not lend itself to such a strategy. In theory either the Cards or the Apples and Oranges problem could be solved using total-minus-bad, however the strategy did not often emerge on those problems. I thus cannot speak to whether my problem choice did or did not facilitate such a strategy ${ }^{46}$.

On 17 occasions students implemented a total-minus-bad strategy in some way, and an additional 7 times students referred to such a strategy. Although the total-minus-bad

\footnotetext{
${ }^{46}$ As a point of interest, I am curious whether total-minus-bad might somehow represent a more sophisticated or robust connection between counting and set theory. My study cannot shed light on such a hypothesis, but a further avenue of study may be to investigate whether any of the set-theoretic principles are hierarchical as they relate to counting. Indeed, total-minus-bad can be conceptualized as the simplest version of the principle of inclusion/exclusion - another important set-theoretic topic.
} 
strategy did not arise as frequently as cases, I believe it to be an important way in which students drew upon set-oriented thinking. In particular, students' abilities to organize according to a total-minus-bad strategy indicate that they can conceive of and organize their total set of outcomes. Being able to situate both desirable and undesirable outcomes within the context of a total number of outcomes strongly suggests a facility with the set of outcomes in question.

\subsection{2 - Students appealed to some specific element of the set of outcomes}

In addition to referring to the entire set of outcomes, students demonstrated setoriented thinking by appealing to particular elements of the set of outcomes. There were a variety of ways in which students appealed to a particular element of the set of outcomes, and I will discuss in detail two major ways in this happened. First, students identified particular elements as they determined or explained whether "order mattered" in a problem. Second, students identified particular elements as they determined or explained an instance of overcounting. This tended to occur when students revisited the problem and when they needed to articulate why a particular expression was too big or too small. I will now further describe some of the ways in which students referred to a particular element of the set of outcomes. Of the 103 instances of student's initial work on the problem, there were 58 instances in which students appealed to some particular outcome (or at least a partial outcome) in some way. However, even if outcomes arose for students as they solved problems and as they subsequently discussed their work with me, they did not always seem to have considered what they were trying to count. On 28 occasions 
students were explicitly asked if they had considered particular examples of what they'd tried to count, and 24 times they said "no." When students revisited the problems, there were 56 recorded instances in which they appealed to a particular outcome.

\subsubsection{1 - Students appeal to a particular element of the set of outcomes in order to make an argument about order mattering or not mattering}

A primary way in which students drew upon a particular element in the set of outcomes was to determine whether or not order mattered for a given situation. As mentioned in the Literature Review chapter, the issue of order is a persistent one for counters. Correctly determining the importance of order in a problem can determine the appropriate course of action for a student on a given problem. Sometimes students rely on keywords to determine if order "matters," or they may have some other automatic mechanism to determine what kind of problem they are dealing with. Order often serves as a primary constraint of a counting problem, and it relates exactly to some of what I found about students' set-oriented perspectives. I claim that an efficient way for a counter to be able to determine whether "order matters" is related to the set of outcomes. Order matters if the counter wants to consider different arrangements of outcomes as being distinct from each other - that is, if arrangements are each counted independently when determining the size of the set. In discussing whether "order matters" in a problem, students referred to particular elements (or to particular features of elements) of the set of outcomes in order to justify why order mattered or not (on 12 occasions in their original work and 8 times as they revisited problems). Indeed, the data supports the fact that 
making sense of order mattering or not mattering can be linked to a discussion of how particular outcomes are the same or different from one another and is thus related to setoriented thinking. When explaining what they meant by "order mattering" (or in explaining why order matters) 20 times students referred to elements of the set of solutions.

An example of students utilizing a particular outcome in order to address an issue of order could be seen in Zach's work on the Groups of Students problem. Zach had initially written down 20 factorial as his solution, but upon reflection he changed it to having four major terms, $\left(\begin{array}{l}20 \\ 5\end{array}\right),\left(\begin{array}{l}15 \\ 5\end{array}\right),\left(\begin{array}{l}10 \\ 5\end{array}\right)$, and $\left(\begin{array}{l}5 \\ 5\end{array}\right)$.

E: And can I ask, so what made you go from, you had the 20 times 19 times 18 times 17 times 16, you felt like maybe that was too big, and then you thought for a little bit and then went to the 20 choose 5 , so what, what uh...

Z: $\quad$ For some reason I mean I just, the, the order of the groups doesn't matter ...

E: Okay, and what do you mean by that?

Z: A Group 1 with ACDEG, this is not distinct from GCDEA, where I swap place of any two students, so I shouldn't need to, that's why the 20 factorial seemed way too big, is you're, you're counting, you're assigning slots for students within the groups, and you don't need to do that. You just need to put them into a group.

E: Okay.

$\mathrm{Z}$ : Um, and then for some reason like once I kind of realized that, it instantly occurred to me well just kind of go with, to, to, to combinations, to, to this formula, it's in the name, 20 choose 5, you're choosing 5 from 20.

At the point of the excerpt, he had not yet decided if he should add or multiply those terms together. I ask him what made him change from 20 factorial (more precisely $20 \cdot 19 \cdot 18 \cdot 17 \cdot 16)$ to $\left(\begin{array}{l}20 \\ 5\end{array}\right)$. His explanation involved an argument about the order of the 
groups not mattering, but, when I ask him what he meant, he appealed to a particular grouping of students (students A, C, D, E, and G). His phrase "this is not distinct from" suggests that he realized he did not actually want to count both ACDEG and GCDEA when he arrived at the total answer. Thus, while it was implicit, he argued using two particular potential elements of the set of outcomes, ultimately deciding that because they were actually not distinct from each other and should not both contribute to the total, order did not matter and he should use the formula for combinations (and not permutations).

Another example of students looking to a particular outcome to determine if order mattered arose in Jon's work on the Apples and Oranges problem. In the excerpt below we see that, with some prompting, he brought up a particular example (apple, apple, orange) in order to determine that order did not matter on this problem.

J: Different nonempty collections can be formed from five identical apples and how many different non-empty collections can be formed from five identical apples and 8 identical oranges, so we're going to have 5 apples and 8 oranges, and we're going to just kind of jumble them all together, so, if they're all five identical apples, and 8 identical oranges, does order matter in this case, because I'm not sure if it matters or not, I don't,

E: Okay, so what, first of all what do you mean by does order matter?

J: Uh, does, like, let's say if we have, so I know they're all identical, but let's say we have apple, orange, apple, would that be the same as saying orange, apple, apple, that's what I'm thinking.

Similar appeals to a particular element in order to determine order occurred throughout the interviews. From these examples we see ways in which students were able to use particular outcomes in their solution set in order to determine whether order 
matters in a problem. Correctly considering order when solving counting problems is a vital aspect of successful counting, and I suggest that a beneficial application of setoriented thinking is that students could use set-oriented thinking to decide whether or not order matters in a given problem. Indeed, in these examples it seems to be the case that order mattering or not mattering is fundamentally linked to the identification of two elements of the set of outcomes, and determining whether those should be counted as the same or different.

In contrast to Zach and Jon's way of determining whether order mattered, recall Kristin's work on the Password's problem in which she explains her decision to use the combination formula on the problem.

E: Okay. Cool and then could you explain maybe one or two of those - the actual terms that you're adding up. So what is that counting for you?

$\mathrm{K}$ : So I'm doing the combination ones, because, um, I'm pretty sure order doesn't matter with combination.

E: Mm-hmm, okay. And why, why do you want order - you don't want order to matter or you do want order to matter?

K: Uh, no, well. No, I don't want order to matter.

E: Okay, and how come?

K: I'm not sure about that one (laughs).

E: Okay.

K: That I just kind of go off my gut for it, on the ones that don't specifically say order matters or it doesn't matter.

In her work on above problem she was trying to determine if order mattered, and she was unsure of whether it mattered or not. However, her ultimate decision was not grounded in the set of outcomes, but rather she went 'off her gut.' I argue that set-oriented thinking 
could help students better ground their decision making about whether order matters in the set of outcomes rather than relying key words or intuition.

\subsubsection{2 - Students appeal to a particular element of the set of outcomes in order to detect, identify or explain an instance of overcounting}

Another fundamental way in which students leveraged the identification of a particular solution occurred when they evaluated two expressions. The study was designed with the purpose of getting at such situations, and the structure of the interviews (specifically, having students solve problems and then evaluate alternative solutions) put students in situations in which they could be faced with two counting processes that seemed similar. The design was effective, and there were 64 instances in which students did indeed evaluate seemingly-identical processes that actually yielded different results.

This activity of evaluating alternative answers fits well into the context of the model presented above. In the model, this activity amounted to giving the students formulas/expressions that looked different (they may or may not have been equivalent) and asking them to determine how the processes those formulas represented related to each other. Then, even further, once students have processes they can evaluate, they could further compare the processes by examining the sets of outcomes related to each process. Thus I believe that the students' work in the evaluation of alternative answers can be represented in the Figure 49 below (note the direction of the arrows). They begin with formulas, connect them to some counting process, and then determine what set of outcomes the counting process has generated. When comparing two such expressions, 
they can ultimately decide if the counting processes that underlie them are the same or different, and then whether those different processes actually count the same set of objects.

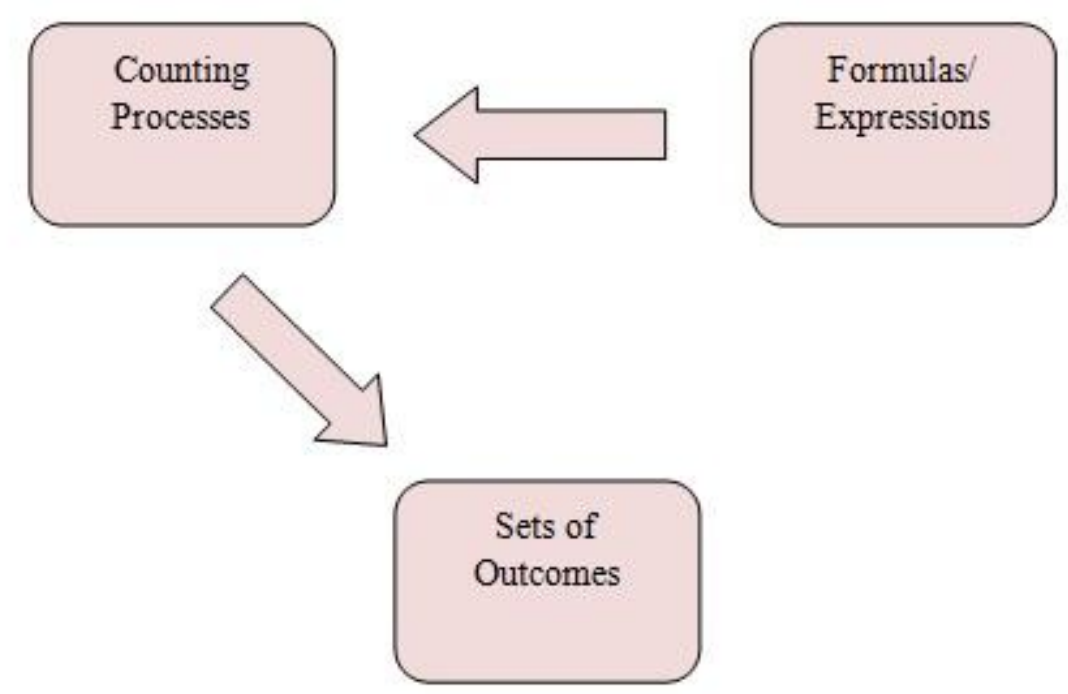

Figure 49

When evaluating alternative expressions, students at times used set-oriented thinking in order to evaluate which expression was correct. In some instances, students thought that the two processes were the same, and some of the analysis that went into their evaluation involved determining whether the two processes did, in fact, differ at all. This happened most commonly on the Passwords problem and the Test Questions problem, as both of these have common incorrect answers whose expressions are not immediately recognizable as different from the correct solutions. Sometimes, though, it was apparent that the two processes were not the same - in the Groups of Students problem, the expressions for the correct and incorrect answers clearly differ by a factor of 4 factorial. 
When evaluating the alternative solutions on this problem, students did not mistake the two answers as being the same (they are clearly not), but they still needed to determine which answer was correct, and why it was correct. Although they did not have to wrestle with the question of whether the processes were the same, as they struggled to justify which solution might be correct, they, at times, considered a particular element that was overcounted in order to realize the error in one of the processes. I will now expound upon this discussion by discussing a number of specific examples from the data.

The example below is representative of a fairly typical occurrence in which a student drew upon a particular element of the set of outcomes in order to identify an overcount (and thus to show why a particular answer was incorrect). This happened 41 times as students revisited problems, and in 15 of those instances I had prompted them to consider a particular element. As mentioned above, inherent in identifying such an element is a coordination of sets and processes - showing that a particular process actually generates an outcome that gets counted too many times (or generates a set of outcomes that contains repeated elements). In Peter's work on the Passwords problem, he had initially gotten the correct answer of

$$
26^{8}-\left[\left(\begin{array}{l}
8 \\
2
\end{array}\right) \cdot 25^{6}+\left(\begin{array}{l}
8 \\
1
\end{array}\right) \cdot 25^{7}+\left(\begin{array}{l}
8 \\
0
\end{array}\right) \cdot 25^{8}\right]
$$

he subtracted off the bad cases correctly. As Peter generated this answer, he did not make reference to any particular outcomes. In fact, when asked if he considered particular passwords, he said, "no." When we revisited this problem in the second half of the interview, I first gave him the other correct case breakdown, 


$$
\left(\begin{array}{l}
8 \\
3
\end{array}\right) \cdot 25^{5}+\left(\begin{array}{l}
8 \\
4
\end{array}\right) \cdot 25^{4}+\left(\begin{array}{l}
8 \\
5
\end{array}\right) \cdot 25^{3}+\left(\begin{array}{l}
8 \\
6
\end{array}\right) \cdot 25^{2}+\left(\begin{array}{l}
8 \\
7
\end{array}\right) \cdot 25^{1}+\left(\begin{array}{l}
8 \\
8
\end{array}\right) \cdot 25^{0}
$$

and he made sense of it. Then, I gave him the common incorrect solution, $\left(\begin{array}{l}8 \\ 3\end{array}\right) \cdot 26^{5}$ to see what he would do.

P: Um, I'm fairly certain that these numbers (the two case breakdowns) will add up correctly just because of the analysis. This one $\left(\begin{array}{l}8 \\ 3\end{array}\right) \cdot 26^{5}$ I wasn't sure at first, I had to sort of convince myself that all the constraints of the problem would be met by this expression. But then once I did, um, I thought oh yeah that's correct. I mean logically, they all seem solid, I would be fine with all of them.

In the excerpt above, we see that he felt that he "logically" made sense of all three answers, and he thought they were all essentially doing the same thing, that they represented equivalent counting processes that should yield the same numerical answers. It is important to note that because of the nature of the expressions, it is not immediately apparent whether these three give the same numerical result, or which expression yields a bigger result, etc.

Peter went on to check the numbers numerically on the calculator, and he realized that $\left(\begin{array}{l}8 \\ 3\end{array}\right) \cdot 26^{5}$ was much bigger (by more than 90 million) than the other two answers. For quite some time he was unsure whether his answer (and the equivalent case breakdown) was wrong, but I indicated to him that he was correct. Thus, he set about trying to determine how the too-big answer might be overcounting.

P: Uh, does that represent an over count? I don't know. Uh, 
E: And so when you're thinking of an over count, what are you trying to, what are you trying to do, or what are you trying to find?

P: I'm trying to find a, uh, I'm trying to find a I guess a situation, let's say like an arrangement of letters,

E: Okay.

P: Where, um, it would only be counted once in an expression like these, but it would be counted twice or more in an expression like this.

The excerpt above suggests that Peter does have a sense of what exactly he needs to do in order to identify an overcount - namely, to find an arrangement of letters that is counted only once by the correct expression, but more than once by the incorrect expression. This represents a coordination of sets and processes, indicating that he can consider that a process generates some outcomes (even if those outcomes are not yet particular examples in his mind).

Peter continued to work for quite some time, and in fact he tried to come up with an element that was counted more than once by the incorrect solution. This was not trivial for him, though, and even after several minutes of work he could not justify why the alternative solution was too big.

P: $\quad$ Maybe that's, that doesn't convince me it's an over count, though. ... So far I haven't been able to convince myself that this is an over count.

Ultimately, after several more minutes of work, Peter did figure out what was wrong with the incorrect expression. In the excerpt below we see that Peter identified a particular password (the all E's password) that was counted more than once, and this convinced him that the incorrect expression was indeed wrong. His activity here gets at the way in which 
the identification of a particular element played a role in helping students explain an instance of overcounting.

P: And then I can sort of arrange those E's wherever I want - those big E's wherever I want them to be. Um, it gives me, so, in other words I can, um, I can, so, so all these, oh, um. Ah, that's it. Okay. Yeah. Yeah, certainly if I have all 8 E's it seems to count it twice, right?

E: Okay, how so?

P: Well, okay so let's say, um, you know I pick the first 3 are going to be E's, and then the last 5, let's say just by random chance, happen to be all 5 E's.

E: Okay.

P: So I've got 8 E's in my thing. Well I could get that same result by picking the last 3 to be E's and then having the first 5 just by random chance being E's as well. So, so this is certainly one, so this is a string that's counted twice, yeah. At least twice, actually more, yeah, more than once. So okay, yeah. That at least convinces me that this $\left[\left(\begin{array}{l}8 \\ 3\end{array}\right) \cdot 26^{5}\right]$ is an overcount.

As mentioned above, there were a total of 41 similar incidents. Students were not always able to determine the correct solution or to figure out why two solutions differed, etc. Invariably though, when students 1) were in the situation of identifying two processes that both logically made sense, and 2) were able to successfully explain/justify which solution was correct and why, they proceeded to identify a particular element. There was only one student (Zach's work on the Password problem) who, in addition to providing an element of the solution set that was overcounted, also attempted to justify the discrepancy using the binomial theorem. His justification using the binomial theorem did not convincingly explain the discrepancy to me, however, and even he said that 
identifying a particular example would be the way he would explain the discrepancy to his students ${ }^{47}$.

As a final aspect of students' identification of an overcount, I discuss two instances that show that set-orienting thinking could actually be ineffective, or even detrimental, when there was an error in the thinking. This may seem obvious, but there were several instances in which students drew upon specific examples, but doing so was either not beneficial for them, or it was disadvantageous for them. First, Joshua's work on the Test Questions problem provided an instance in which a student came up with a particular outcome, but it was not one that could help illuminate why a particular answer was wrong. In the Test Questions problem, an outcome that is overcounted by a solution $\left(\begin{array}{l}5 \\ 2\end{array}\right) \cdot\left(\begin{array}{l}8 \\ 3\end{array}\right)$ is one in which more than 2 of the first 5 questions are chosen. As described in the Results chapter, Joshua had been comparing two solutions:

$$
\left(\begin{array}{l}
5 \\
2
\end{array}\right) \cdot\left(\begin{array}{l}
5 \\
3
\end{array}\right)+\left(\begin{array}{l}
5 \\
3
\end{array}\right) \cdot\left(\begin{array}{l}
5 \\
3
\end{array}\right)+\left(\begin{array}{l}
5 \\
4
\end{array}\right) \cdot\left(\begin{array}{l}
5 \\
1
\end{array}\right)+\left(\begin{array}{l}
5 \\
5
\end{array}\right) \cdot\left(\begin{array}{l}
5 \\
0
\end{array}\right)
$$

and $\left(\begin{array}{l}5 \\ 2\end{array}\right) \cdot\left(\begin{array}{l}8 \\ 3\end{array}\right)$. He wanted to come up with an example that was counted too many times by $\left(\begin{array}{l}5 \\ 2\end{array}\right) \cdot\left(\begin{array}{l}8 \\ 3\end{array}\right)$. When I prompted him to write a particular outcome, he came up with the set of questions $\{1,2,6,7,8\}$. While this is indeed one of the desirable outcomes, it was not a problematic outcome. Therefore, at that time his choice of outcome did not cause him

\footnotetext{
${ }^{47}$ Zach had been a high school mathematics teacher for several years.
} 
to see the problem with the incorrect solution. It was not until he later came up with the example of $\{1,2,3,4,5\}$, a set of questions that was overcounted by $\left(\begin{array}{l}5 \\ 2\end{array}\right) \cdot\left(\begin{array}{l}8 \\ 3\end{array}\right)$, that he could detect the error in the incorrect expression.

Second, I highlight what can go wrong when a particular outcome is not correctly identified early on in a students' work (or, more pointedly, when a particular outcome is incorrectly identified). An example of this arose in Kristin's work on the Test Questions problem. Early in her work on the problem she had articulated what she believed she was counting, and she placed $x$ 's above dashes to represent a particular set of questions that had been answered, as in Figure 50 below.

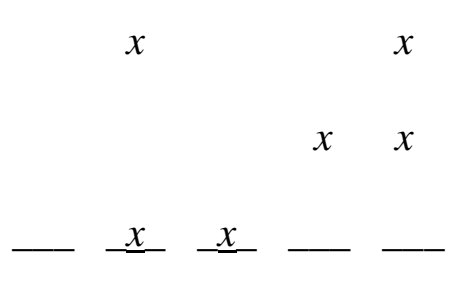

Figure 50

Unfortunately, however, she had an incorrect notion of what constituted "different" and "same" outcomes. She stated that she wanted to consider two elements as the same which really should have been different. Specifically, she was mistaking the actual location of the $x$ 's (representing a set of questions answered) for the order in which the locations were chosen. And, it was not the case that she simply misspoke - I asked her subsequently in the interview about what she was trying to count, and she again talked 
about her particular outcomes incorrectly ${ }^{48}$. The negative impact of this understanding of what she was trying to count was undeniable. In addition to getting the problem incorrect initially, because of her incorrect notion of what she was trying to count, she could not easily make sense of alternative solutions, and ultimately she could not make meaningful headway on the problem. Thus, we see from these examples that set-oriented thinking alone will not necessarily give students particular insight into a counting problem; it is not just a matter of using set-oriented thinking, but rather it is a matter of correctly and strategically using it.

In addition to the different ways in which students appealed to particular outcomes, as discussed above, there were a number of contexts in which the particular outcomes were identified - specifically when working on the original problem, when working through a smaller case, and when engaging in systematic listing.

On certain occasions, students came up with a particular outcome in the context of the original problem. We saw this in Peter's work on the Passwords problem. I had prompted him to consider why one answer was bigger than another, and after some time he identified the all E's password as being overcounted. This was a case in which just considering the original problem seemed to be sufficient for him to come up with a particular example. This happened 11 times in the students' original work on the problem. As students revisited the problems, there were 41 instances in which students

\footnotetext{
${ }^{48}$ This is found in the Results chapter in the second excerpt in Kristin's work on the Test Questions problem
} 
came up with a particular solution in order to determine an over count (11 of those instances were prompted).

Other students came up with particular outcomes within the contexts of smaller cases. For example, on the Passwords problem, in order for Anderson to explain the difference between two expressions that he thought were the same, he went to a smaller case. While he worked through the smaller version of the problem, he wrote out some cases, and he applied versions of the original expressions that would be appropriate in the smaller case. His listing of E E E A-Z and E E A-Z E gave him insight into the structure of his outcomes, and in the context of the smaller case, the listing enabled him to identify the discrepancy between the two expressions. Noting a numerical difference even in the smaller instance, he identified a particular element that was counted too many times by one expression. This allowed him to see which expression was correct in the original statement of the problem.

Owen provided a similar example in which a smaller case and some careful identification of a particular element were helpful. Below we recall how he specifically used color coding (Figure 51) in order to articulate a way in which an element was generated too many times, thus causing an over count. 


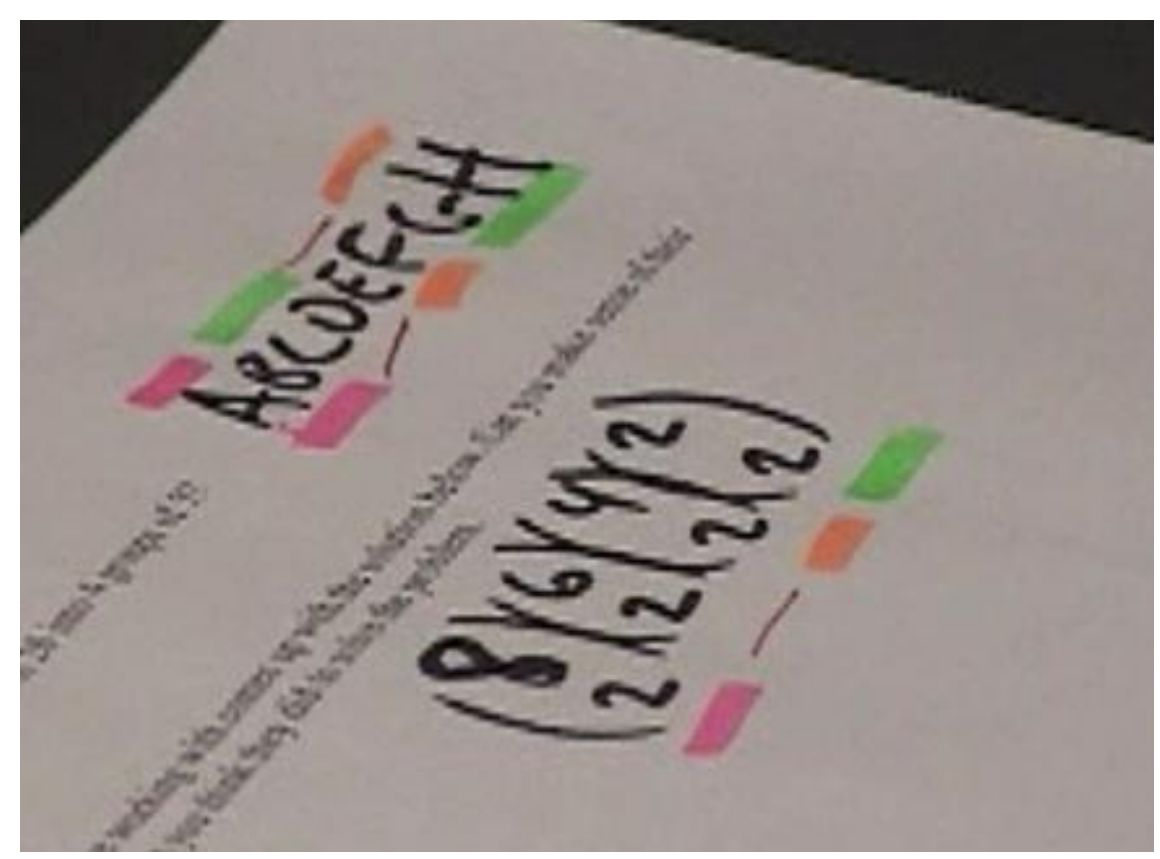

Figure 51

Even though identifying a problematic outcome within a smaller case was effective for Anderson and Owen, the strategy was not used very often (there were a total of only 6 instances). This is thus an example of a useful but atypical way in which students identified an over count.

There were also times in which particular outcomes were articulated as students engaged in the activity of systematic listing - this occurred within the context of the original problem, but it also occurred within smaller cases. Brandon's work on the Passwords problem shows an example in which he wrote a partial systematic list in order to determine the number of ways of placing E's. While he did not write out entire passwords, and while his list was not complete (he used some shortcuts), the fact that he started to list elements in an organized way suggests that he considered the outcomes he tried to count. He was able to give a sense of what the elements of his set of outcomes 
might look like, and he used their properties to enumerate what he wanted. We saw Makaena, too, in the Groups of Students problem, engage in a similar activity within the context of a smaller case.

M: Sure. Okay. So I thought about, well, let's look at a smaller case, say I have 6 people, so I listed them out, ABCDE and F, drew my little picture and then went with the method that I chose, um, when I first did the problem, and so, uh, 6 choose 3 and that leaves me with 3 choose 3 ,

E: Okay.

M: Uh, and I got 20, and then I thought, okay I want to compare that to what this method does [the method in Expression R], which is then to divide out by the number of ways to arrange those groups of people. So here it was 4 factorial, here it would be 2 factorial, which is 2 , so 20 versus 10 . So I started making these theoretical groups, um, and as I was listing out thinking, oh I don't want to list them all out, what would happen later on in the alphabet? You know it wouldn't just be A, B, and C. Later on I might get a DEF in the first group, but then that forces $\mathrm{ABC}$ in the second group. Um, and then I realized oh, that's the same thing here, and I, using this method it counts them each separately as one, uh, this is one way, this is another way, but they aren't really. So, um, this [referring to ABC $\underline{\mathrm{DEF}}$ ] would be double counted. And then let's say, so then here, it's not just halving it, like I don't think it would make sense just to divide this by 2 ,

She had gone to a smaller version of the problem, and she started to list out some ways in which to divide 6 students into 2 groups of 3 . As she engaged in systematic listing, she realized that she had written down two ways to split up students that were actually the same. In Makaena's case, it seems clear that her act of systematically listing had a direct bearing on her success on the problem - that by listing she could identify the way in which duplicate outcomes were being generated. She was able to relate her work on the small example to the original problem. I recorded a total of 33 instances of systematic listing in the students' original work on the problem, 14 of which were error-free. There 
were also 3 instances of systematic listing as students revisited problems, 2 of which were error-free.

I have one final noteworthy point about students' references to particular elements of the set of outcomes. On a number of occasions, students provided partial examples of a particular outcome. For example, In Marcus' work on the Passwords problem, when I prompted him to give some examples, he gave the following: $\mathrm{E}_{-} \mathrm{E}_{-} \mathrm{E}_{-}{ }_{-}$. While this example is not technically a particular element of the set of outcomes (it is not actually an 8-letter password), it is a partial element, and when students alluded to outcomes in this way, this suggested to me that they were engaging in set-oriented thinking (they were within the realm of thinking about outcomes in some way). It could be the case that students could refer to partial outcomes with varying levels of sophistication. That is, students could knowingly refer to a partial outcome that could actually represent a class of outcomes - I would refer to such an outcome as a "prototypical outcome." Or, students could write a partial outcome because they do not know how to correctly express an element of the set of outcomes. In these cases, their understanding of the set of outcomes may be incomplete. I did not carefully investigate the extent to which these instances arose in my study (and in particular I did not delineate between sophisticated or unsophisticated references to partial outcomes), but it is worth mentioning and could be an avenue for further study. Another example of this was in Brandon's work on the Passwords problem, in which Brandon began the process of partially listing out some prototypical outcomes. He did not systematically list complete passwords, but he 
systematically listed the places in which the E's were placed. Students referred to incomplete or partial elements 47 times throughout the initial attempts.

This concludes our discussion of students' uses of particular outcomes in their setoriented thinking. In sum, the study was, in many ways, designed in order to investigate exactly the phenomenon described in this section - to answer the question of what students would do in order to identify and explain differences between two seemingly identical processes. One major question I had coming into the study was whether I would learn new ways in which students could get out of this situation and explain the subtleties of why the expressions differed. However, it did not seem to be the case that students typically drew upon anything other than identifying a particular element that was overcounted. It therefore seems that being able to identify a particular element provides one specific way in which set-oriented thinking can help one detect and justify an error of overcounting.

As mentioned at the end of Section 5.2, in a grounded theory study, conclusions include hypotheses which could be tested in future studies. In this section I have described ways in which I observed students utilizing set-oriented thinking as they solved counting problems, and I have pointed out the benefit that such thinking conferred. One major theme that has come out of the findings described in this section is that considering the set of outcomes is an important aspect of successful counting. That is, it seems as though drawing upon the set of outcomes can be a powerful tool for students in order to maximize their chances at correctly solving counting problems. Thus, a hypothesis that comes out of this section is that sets of outcomes may be a necessary element of helping 
students to be successful in certain counting situations. Said another way, there exist circumstances in which students will not able to make progress if they do not connect counting processes and/or formulas to sets of outcomes. That is, while there are some counting problems for which students can be successful without drawing upon sets of outcomes, the consideration of sets of outcomes is indispensable in order to correctly deal with or make sense of some counting situations (such as the evaluation of seeminglysimilar counting processes).

\section{4 - Avenues for further study}

The fact that this study was designed and analyzed through the methodological lens of grounded theory suggests that my findings described above are an initial step on which further research studies can be based. Indeed, while I believe that the model of coordinating formulas/expressions, counting processes and sets of outcomes is a useful theoretical contribution, it was developed in the final stages of analysis. It emerged from my analysis of the data, and I have not had the chance to investigate the extent to which it might be useful as a theoretical framework to further investigate students' work on counting problems. In this section I elaborate some specific potential ways in which this model and some of the other findings could lead to subsequent research projects.

First, my conclusions call for a deeper investigation of the model I have presented above. There are two clear avenues to pursue. On the one hand, researchers could focus on better understanding the model as it is now, particularly trying to use it as a lens through which to conduct research on students' understanding of counting. To be more 
specific, I could envision using the model to describe students' combinatorial thinking and activity on a given problem, or perhaps as a way to situate various approaches to a given problem. This might include discussing theoretical approaches to a problem, or analyzing a particular student's work on a problem, in terms of the model. And, too, my work has focused primarily on the relationship between counting processes and sets of outcomes, and I believe that there is potential in exploring some of the other components and relationships more deeply. There may be broader notions for what could be included in the formula/expressions component, for example, or there may be value in describing the relationship between sets of outcomes and formulas/expressions.

Second, I believe the study emphasizes the importance of coordinating counting processes with the set of outcomes; that is, I have evidence of ways in which set-oriented thinking was beneficial for students in their counting. The model has helped to make clear the importance of such coordination, and this could lead the research community to pursue a couple of avenues of research on this topic. First, this coordination could be investigated as an important element of students' learning and thinking about counting, and it could thus be elaborated from a cognitive perspective. Second, researchers could strive to learn more about how instructors might foster such coordination among counting students. Specifically, there could be great value in designing studies that explore ways in which students could effectively be taught to utilize the coordination of sets and processes as they count.

Third, the study suggests that there are potentially naturally occurring relationships between counting and formal set theory, and I suggest that research could be done that 
more clearly investigates the value of formal set theory for counting students. There are set theoretic ideas that are embedded in much of the set-oriented thinking I have discussed thus far - cases are related to ideas like partitions and disjoint subsets, for example, and identifying a particular outcome that was overcounted has to do with the fact that sets, by definition, do not contain repeated elements. I propose that there could be value in investigating whether other naturally occurring relationships exist between counting and explicit set theory, and whether these could be leveraged for students' benefit as they count.

Fourth, the study suggests promising avenues of research in the area of student work with combinatorial proof. Combinatorial proof involves the proving of combinatorial identities; it is a topic that has been studied by some mathematics education researchers (e.g., Engelke \& CadwalladerOlsker, 2010), but not widely and not in depth. A combinatorial proof, by definition, involves proving an equation by showing that each side of the equation counts the same set of objects. As an example, let us consider a simple combinatorial identity. Suppose we want to prove combinatorially that for all positive integers $n$ and $k$,

$$
\left(\begin{array}{l}
n \\
k
\end{array}\right)=\left(\begin{array}{l}
n-1 \\
k-1
\end{array}\right)+\left(\begin{array}{l}
n-1 \\
k
\end{array}\right) .
$$

In order to prove this, we argue that the left side counts the number of committees of size $k$ chosen from $n$ people, which can be done simply by choosing $k$ people from $n$. The right hand side is also going to count the number of committees of size $k$ chosen from $n$ people, but we arrive at that number by breaking the number of such committees into two 
distinct types - those that include one of the people (call him Tom) and those that do not. If Tom is on the committee, then there are $n-1$ other people available to choose for the committee, and we must choose $k-1$ of those people to join Tom. If Tom is not on the committee, then we still have $n-1$ people to choose from (excluding Tom), and we want $k$ of them on the committee. This example shows that combinatorial proof necessarily involves a discussion of two different ways of counting the exact same set of objects. In our example, the set of objects was size- $k$ committees chosen from $n$ people, and we argued through two different ways of enumerating that set. The proving of combinatorial identities is a unique but important aspect of proof and of combinatorics. My findings on the coordination of sets and processes and on students' set-oriented thinking could be valuable resources for a study on combinatorial proof. I thus propose that the study of combinatorial identities and combinatorial proof is an avenue for further study in light of my research, because, by its nature, combinatorial proof explicitly investigates multiple ways to enumerate the cardinality of a set.

Fifth, the study indicates that a researcher could more closely examine how the model presented above could relate to some of the existing theories outlined in Section 2.2. Since I have, in the model, examined relationships between counting processes and sets of outcomes, it may be the case that the model could be further investigated through the lens of considering Actions, Processes, Objects, and Schemes (called APOS theory, Dubinsky \& McDonald, 2001) or of Sfard's (1991) notion of structural and operational conceptions. In particular, in APOS theory, the notion of the encapsulation of a process to an object could give a useful framework for investigating the relationship between a 
counting process and formulas/expressions. It could be worth investigating whether or not (and if so, in what ways) counters could gain flexibility if they could encapsulate their counting processes as objects and consider operations on those objects. A study examining such conjectures could be a worthwhile endeavor and could shed light on important aspects of students' counting. That is, APOS could be a lens through which subsequent studies on students' set-oriented thinking are designed and conducted. Similarly, Sfard's (1991) complementary approach to the structural and operational relationship is something that could be highlighted in further studies. I conjecture that the relationship between counting processes and sets of outcomes in my model is more complementary than dichotomous, and Sfard's work could likely serve as a valuable framework through which to further investigate the model. Indeed, a promising avenue for further study could be to investigate framing my findings here in terms of Sfard's notions of structural and operational conceptions.

In sum, my findings call for a number of research studies that could contribute to the further understanding of how students conceptualize, approach, and solve counting problem. Specifically, there is room to investigate further the model described in this chapter. There are also opportunities to investigate effective ways in which to foster the coordination of sets and processes, to ground students' work in combinatorial proof, to orient students' counting practices in the set of outcomes, and to relate the model to existing theories in mathematics education. 


\section{5 - Summary of the Conclusions chapter}

To conclude, I recall the ways in which my research questions were addressed through the findings put forth in this chapter. First, I reminded the reader of the ways in which model that had been introduced in Section 4.1. This model had served as a conceptual framework that was rooted in mathematics but that had been refined through analysis of the data. I was able to situate my major findings within the model. Next, I gave examples of ways in which students engaged in activity that did not suggest setoriented thinking; specifically, they solved counting problems by focusing only on the relationship between counting processes and formulas/expressions, not appealing to sets of outcomes in any way. Then, I gave a detailed description of an emerging framework for the various ways in which students used set-oriented thinking to their benefit as they solved counting problems. I made a distinction between students' references to the set of outcomes as a whole and to students' appeals to particular outcomes, and I explained particular ways in which I observed set-oriented thinking throughout the students' work. This was tied to my results, and I provided specific examples from the data and also gave numerical tallies where appropriate. I ended the chapter with a discussion of ideas for further avenues of study, offering several ideas for specific studies that could build upon my findings. 


\section{References}

Abramovich, S., \& Pieper, A. (1996). Fostering recursive thinking in combinatorics through the use of manipulatives and computer technology. The Mathematics Educator, 7(1), 4-12.

Althoen, S. C., Brown, J. L., \& Bumcrot, R. J. (1991). Graph chasing across the curriculum: Paths, circuits, and applications. In M. J. Kenney \& C. R. Hirsch (Eds.), Discrete Mathematics across the Curriculum, K-12: 1991 Yearbook (pp. 30-43). Reston, VA: National Council of the Teachers of Mathematics.

Asiala, M., Brown, A., DeVries, D. J., Dubinsky, E., Mathews, D., Thomas, K. (2004). A framework for research and curriculum development in undergraduate mathematics education. Research in Collegiate Mathematics Education II, CBMS Issues in Mathematics Education, 6, 1-32.

Auerbach, C. \& Silverstein, L. B. (2003). Qualitative data: An introduction to coding and analysis. New York: New York University Press.

Batanero, C., Godino, J., \& Navarro-Pelayo, V. (1997). Combinatorial reasoning and its assessment. In I. Gal \& J. B. Garfield (Eds.), The Assessment Challenge in Statistics Education (pp. 239-252): IOS Press.

Batanero, C., Navarro-Pelayo, V., \& Godino, J. (1997). Effect of the implicit combinatorial model on combinatorial reasoning in secondary school pupils. Educational Studies in Mathematics, 32, 181-199.

Bona, M. (2007). Introduction to Enumerative Combinatorics. New York: McGraw Hill.

Brualdi, R. A. (2004). Introductory Combinatorics (4 ${ }^{\text {th }}$ ed.). Upper Saddle River, New Jersey: Pearson Prentice Hall.

Cameron, P. J. (1994). Combinatorics: Topics, Techniques, Algorithms. Cambridge: Cambridge University Press.

Carlson, M. P. (1999). The mathematical behavior of six successful mathematics graduate students: Influences leading to mathematical success. Educational 
Studies in Mathematics, 40, 237-258.

Carlson, M. P. \& Bloom, I. (2005). The cyclic nature of problem solving: an emergent multidimensional problem-solving framework. Educational Studies in Mathematics, 58, 45-75.

Charmaz, K. (2006). Constructing Grounded Theory: A Practical Guide through Qualitative Analysis. Thousand Oaks, California: Sage Publications, Inc.

Clement, J. (2000). Analysis of clinical interviews: foundations and model viability. In A. E. Kelly \& R. Lesh (Eds.), Handbook of research data design in mathematics and science education (pp. 547-589). Mahwah, NJ: Lawrence Erlbaum Associates.

DeBellis, V., \& Rosenstein, J. G. (2004). Discrete mathematics in primary and secondary schools in the United States. ZDM, 36, 77-81.

DeGuire, L. J. (1991). Permutations and combinations: A problem-solving approach for middle school students. In M. J. Kenney \& C. R. Hirsch (Eds.), Discrete Mathematics across the Curriculum, K-12: 1991 Yearbook (pp. 59-66). Reston, VA: National Council of Teachers of Mathematics.

Dossey, J. A. (1991). Discrete mathematics: The math for our time. In M. J. Kenney \& C. R. Hirsch (Eds.), Discrete Mathematics across the Curriculum, K-12: 1991 Yearbook (pp. 1-9). Reston, VA: National Council of Teachers of Mathematics.

Dubois, J. G. (1984). Une systematique des configurations combinatoires simples. Educational Studies in Mathematics, 15(1), 37-57.

E. Dubinsky. A theory and practice of learning college mathematics, in (A. Schoenfeld, ed.) Mathematical Thinking and Problem Solving. Hillsdale: Erlbaum, 221-243, 1994.

Dubinsky, E., \& McDonald, M. A. (2001). APOS: A constructivist theory of learning in undergraduate mathematics education research. In D. Holton (Ed.), The Teaching and Learning of Mathematics at University Level: An ICMI Study. Series: New ICMI Study Series, Vol.7 (pp. 273-280). Dordrechet: Kluwer. 
Ebert, C., Ebert, G., \& Klin, M. (2004). From the principle of bijection to the isomorphism of structures: An analysis of some teaching paradigms in discrete mathematics. ZDM, 36, 172-183.

Eizenberg, M. M., \& Zaslavsky, O. (2003). Cooperative problem solving in combinatorics: The inter-relations between control processes and successful solutions. Journal of Mathematical Behavior, 22, 389-403.

Eizenberg, M. M., \& Zaslavsky, O. (2004). Students' verification strategies for combinatorial problems. Mathematical Thinking and Learning, 6(1), 15-36.

Engelke, N. and CadwalladerOlsker, T. (2010.) Counting Two Ways: The Art of Combinatorial Proof. Proceedings of the Thirteenth SIGMAA on RUME Conference on Research in Undergraduate Mathematics Education.

English, L. D. (1991). Young children's combinatorics strategies. Educational Studies in Mathematics, 22, 451-47

English, L. D. (1993). Children's strategies for solving two- and three-dimensional combinatorial problems. Journal of Mathematical Behavior, 24(3), 255-273.

English, L. D. (2005). Combinatorics and the development of children's combinatorial reasoning. In G. A. Jones (Ed.), Exploring probability in school: Challenges for teaching and learning (Vol. 40, pp. 121-141): Kluwer Academic Publishers.

Evered, L. J., \& Schroeder, B. (1991). Counting with generating functions. In M. J. Kenney \& C. R. Hirsch (Eds.), Discrete Mathematics across the Curriculum, $K$ 12: 1991 Yearbook (pp. 143-149). Reston, VA: National Council of Teachers of Mathematics.

Fischbein, E., \& Gazit, A. (1988). The combinatorial solving capacity in children and adolescents. ZDM, 5, 193-198.

Fischbein, E., \& Grossman, A. (1997). Schemata and intuitions in combinatorial reasoning. Educational Studies in Mathematics, 34, 27-47. 
Fischbein, E., Nello, M. S., \& Marino, M. S. (1991). Factors affecting probabilistic judgements in children and adolescents. Educational Studies in Mathematics, 22(6), 523-549.

Fischbein, E., \& Schnarch, D. (1997). The evolution with age of probabilistic, intuitively based misconceptions. Journal for Research in Mathematics Education, 28(1), 96-105.

Garofalo, J., \& Lester, F. K. (1985). Metacognition, cognitive monitoring, and mathematical performance. Journal for Research in Mathematics Education,16, 163-176

Gardiner, A. D. (1991). A cautionary note. In M. J. Kenney \& C. R. Hirsch (Eds.), Discrete Mathematics across the Curriculum, K-12: 1991 Yearbook (pp. 10-17). Reston, VA: National Council of Teachers of Mathematics.

Glass, B., \& Maher, C. A. (2002, July 21-26, 2002). Comparing representations and reasoning in young children with two-year college students. Paper presented at the 26th Annual Meeting of the International Group for the Psychology of Mathematics Education, Norwich, England.

Glass, B., \& Maher, C. A. (2004). Students' problem solving and justification. Paper presented at the 28th Conference of the International Group for the Psychology of Mathematics Education.

Godino, J., Batanero, C., \& Roa, R. (2005). An onto-semiotic analysis of combinatorial problems and the solving processes by university students. Educational Studies in Mathematics, 60, 3-36.

Grenier, D., \& Payan, C. (1999). Discrete mathematics in relation to learning and teaching proof and modeling. Paper presented at the Conference of the European Society for Research in Mathematics Education (CERME-1), Onsabruck.

Hadar, N., \& Hadass, R. (1981). The road to solve combinatorial problems is strewn with pitfalls. Educational Studies in Mathematics, 12, 435-443. 
Hasenbank, J. F. (2006). The effects of a framework for procedural understanding on college algebra students' procedural skill and understanding. Unpublished doctoral dissertation, Montana State University - Bozeman, MT.

Heitele, D. (1975). An epistemological view on fundamental stochastic ideas. Educational Studies in Mathematics, 6(2), 187-205.

Hiebert, J. (1992). Mathematical, cognitive, and instructional analyses of decimal fractions. In G. Leinhardt, R. Putnam, \& R. Hattrup (Eds.), Analysis of arithmetic for mathematics teaching (pp. 2283-322). Hillsdale, NJ: Lawrence Erlbaum.

Hiebert, J., \& Lefevre, P. (1986). Conceptual and procedural knowledge in mathematics: An introductory analysis. In J. Hiebert (Ed.), Conceptual and procedural knowledge: The case of mathematics (pp. 1-27). Hillsdale, NJ: Lawrence Erlbaum.

Holliday, R. L. (1991). Graph theory in the high school curriculum. In M. J. Kenney \& C. R. Hirsch (Eds.), Discrete Mathematics across the Curriculum, K-12: 1991 Yearbook (pp. 87-95). Reston, VA: National Council of Teachers of Mathematics.

Jones, G. A., Langrall, C. W., Thornton, C. A., \& Mogill, A. T. (1999). Students' probabilistic thinking in instruction. Journal for Research in Mathematics Education, 30(5), 487-519.

Kapur, J. N. (1970). Combinatorial analysis and school mathematics. Educational Studies in Mathematics, 3(1), 111-127.

Kaput, J. (1992). Patterns in students' formalization of quantitative patterns. In G. Harel, \& E. Dubinsky (Eds.), The concept of function: Aspects of epistemology and pedagogy (pp. 290-317). Washington, DC: Mathematical Association of America.

Kavousian, S. (2008) Enquiries into undergraduate students' understanding of combinatorial structures. Unpublished doctoral dissertation, Simon Fraser University - Vancouver, BC. 
Kenney, M. J., \& Hirsch, C. R. (Eds.). (1991). Discrete mathematics across the curriculum, K-12: 1991 Yearbook. Reston, VA: National Council of Teachers of Mathematics.

Kieran, C. (1992). The learning and teaching of school algebra. In D. Grouws (Ed.), Handbook of Research on mathematics teaching and learning (pp. 390-419). New York, NY: Macmillan

Kilpatrick, J. (1985). A Retrospective Account of the Past Twenty-five Years of Research on Teaching Mathematical Problem Solving, in Silver, (Ed.), Teaching and learning mathematical problem solving: Multiple research perspectives (pp. 115). Hillsdale, NJ: Erlbaum.

Konold, C., Pollatsek, A., Well, A., Lohmeier, J., \& Lipson, A. (1993). Inconsistencies in students' reasoning about probability. Journal for Research in Mathematics Education, 24(5), 392-414.

Lester, F. K., Garafalo, J., \& Kroll, D. L. (1989). Self-confidence, interest, beliefs, and metacognition: Key influences on problem-solving behavior. In D. B. McLeod and V. M. Adams (Eds.), Affect and Mathematical Problem Solving: A New Perspective (pp. 75-88). New York: Springer-Verlag.

Lipton, Jennifer S (01/01/2006). "Preschool Children Master the Logic of Number Word Meanings". Cognition (0010-0277), 98 (3), p. B57.

Lobato, J. (2003). How design experiments can inform a rethinking of transfer and vice versa. Educational Researcher, 32(1), 17-20.

Lockwood, E. (2009). Investigation student approaches to counting problems: An exploration using the notion of actor-oriented transfer. The Electronic Proceedings of the $12^{\text {th }}$ Annual Meeting of the Research on Undergraduate Mathematics Education. Raleigh, NC: North Carolina State University.

Lockwood, E. (2010). An investigation of post-secondary students' understanding of two fundamental counting principles. The Electronic Proceedings of the $13^{\text {th }}$ Annual Meeting of the Research on Undergraduate Mathematics Education. Raleigh, NC: North Carolina State University. 
Lockwood, E. (2011). Student connections among counting problems: an exploration using actor-oriented transfer. Educational Studies in Mathematics. doi: 10.1007/s10649-011-9320-7.

Maher, C. A. (2002, July 21-26, 2002). How students structure their own investigations and educate us: What we've learned from a fourteen year study. Paper presented at the 26th Annual Meeting of the International Group for the Psychology of Mathematics Education, Norwich, England.

Maher, C. A., \& Martino, A. (1996). Young children invent methods of proof: The gang of four. In P. Nesher, L. Steffe, P. Cobb, B. Greer \& J. Goldin (Eds.), Theories of Mathematical Learning (pp. 431-447). Manwah, NJ: Lawrence Erlbaum Associates.

Maher, C. A. \& Speiser, R. (2002). How far can you go with block towers? The Journal of Mathematical Behavior, 16, 2, 125-132.

Mamona-Downs, J. \& Downs, M. (2004). Realization of techniques in problem solving: the construction of bijections for enumeration tasks. Educational Studies in Mathematics, 56, 235-253.

Martin, G. E. (2001). The Art of Enumerative Combinatorics. New York: Springer.

Martino, A. M., \& Maher, M. A. (1994). Teacher questioning to stimulate justification and generalization in mathematics. Paper presented at the Annual Meeting of the American Educational Research Association.

Maxwell, J. A. (2002). Understanding and validity in qualitative researchers. In A. M. Huberman \& M. B. Miles (Eds.) The Qualitative Researcher's Companion (pp 37-64). Thousand Oaks, CA: Sage Publications, Inc.

Mellinger, K. E. (2004). Ordering elements and subsets: Examples for student understanding. Mathematics and Computer Education, 38(3), 333-337.

Miller, D. W. (1991). Discovering and applying Euler's formula. In M. J. Kenney \& C. R. Hirsch (Eds.), Discrete Mathematics across the Curriculum, K-12: 1991 
Yearbook (pp. 96-103). Reston, VA: National Council of Teachers of Mathematics.

National Council of Teachers of Mathematics. (1989). Curriculum and evaluation standards for school mathematics. Reston, VA: NCTM.

National Council of Teachers of Mathematics. (2000). Principles and standards for school mathematics. Reston, VA: NCTM.

Piaget, J., \& Inhelder, B. (1975). The origin of the idea of chance in children. New York: W. W. Norton \& Company, Inc.

Pirie, S., \& Kieren, T. (1994). Growth in mathematical understanding: How can we characterize it and how can we represent it? Educational Studies in Mathematics, 26, 165-190.

Powell, A. B., \& Maher, C. A. (2003). Heuristics of twelfth graders building isomorphisms. In N. A. Pateman, B. J. Dougherty \& J. T. Zilliox (Eds.), Proceedings of the 2003 Joint Meeting of PME and PMENA (Vol. 4, pp. 23-30). Honolulu: CRDG, College of Education, University of Hawai'i.

Powell, A. B., Francisco, J. M. \& Maher, C. A. (2003). An analytical model for studying the development of learners' mathematical ideas and reasoning using videotape data. Journal of Mathematical Behavior, 22, 403-435.

Polya, G. (1957). How to solve it: A new aspect of mathematical method ( $2^{\text {nd }}$ ed.). Garden City: Doubleday.

Rittle-Johnson, B., Siegler, R.S. \& Alibali, M.W. (2001). Developing conceptual understanding and procedural skill in mathematics: An iterative process. Journal of Educational Psychology, 93, 346-362.

Roa, R. (2000). Razonamiento combinatorio en estudiantes con preparacion matematica avanzada. Unpublished Doctoral dissertation. Universidad de Granada.

Roberts, F. S. \& Tesman, B. (2005). Applied Combinatorics $\left(2^{\text {nd }}\right.$ ed.). Upper Saddle River, New Jersey: Pearson Prentice Hall. 
Rosen, K. H. (2007). Discrete Mathematics and Its Applications (6 ${ }^{\text {th }}$ ed.). New York: McGraw Hill.

Rubel, L. (2007). Students' probabilistic reasoning. Journal of Mathematical Behavior, $38(5), 531-556$.

Scardamalia, M. (1977). Information processing capacity and the problem of horizontal decalage: A demonstration using combinatorial reasoning tasks. Child Development, 48, 28-37.

Schielack, J. F. (1991). Primary experiences in learning what (as well as how) to count. In M. J. Kenney \& C. R. Hirsch (Eds.), Discrete Mathematics across the Curriculum, K-12: 1991 Yearbook (pp. 44-50). Reston, VA: National Council of Teachers of Mathematics.

Schliemann, A., \& Acioly, N. (1989). Mathematical knowledge developed at work: The contribution of practice versus the contribution of schooling. Cognition and Instruction, 6(3), 185-221.

Schoenfeld, A. H. (1985). Problem solving in context(s). In E. A. Silver (Ed.), The Teaching and Assessing of Mathematical Problem-Solving, Vol. 3. Reston, VA.

Schoenfeld, A.H. (1992). Learning to think mathematically: Problem solving, metacognition and sense-making in mathematics. In D. A. Grouws (Ed.) Handbook for Research on Mathematics Teaching and Learning,(pp 334-370). New York: Macmillan Publishing Company.

Schuster, A. (2004). About traveling salesmen and telephone networks - combinatorial optimization problems at high school. ZDM, 36, 77-81.

Sfard, A. (1991). On the dual nature of mathematical conceptions: Reflections on processes and objects as different sides of the same coin. Educational Studies in Mathematics, 22, 1-36.

Sfard, A. (1992). Operational origins of mathematical objects and the quandary of reification. The case of function. In G. Harel, \& E. Dubinsky (Eds.), The concept 
of function: Aspects of epistemology and pedagogy (pp. 59-84). Washington, DC: Mathematical Association of America

Shaughnessy, J. M. (1977). Misconceptions of probability: An experiment with a smallgroup, activity-based, model building approach to introductory probability at the college level. Educational Studies in Mathematics, 8, 295-316.

Spangler, D. A. (1991). The pigeonhole principle: A counting technique for the middle grades. In M. J. Kenney \& C. R. Hirsch (Eds.), Discrete Mathematics across the Curriculum, K-12: 1991 Yearbook (pp. 55-58). Reston, VA: National Council of the Teachers of Mathematics.

Sriraman, B., \& English, L. D. (2004). Combinatorial mathematics: Research into practice. Mathematics Teacher, 98(3), 182-191.

Stanley, R. (1997). Enumerative Combinatorics, Volume I. Cambridge: Cambridge University Press.

Star, J. R. (2005). Reconceptualizing procedural knowledge. Journal for Research in Mathematics Education, 36 (5), 404-411.

Strauss, A. \& Corbin, J. (1998). Basics of Qualitative Research: Techniques and Procedures for Developing Grounded Theory (2 ${ }^{\text {nd }}$ ed.). Thousand Oaks, California: Sage Publications, Inc.

Thompson, P. \& Saldanha, L. (2002). Conceptions of sample and their relationship to statistical inference. Educational Studies in Mathematics, 51, 257-270.

Tillema, E. (2007). Students' construction of algebraic symbol systems. Unpublished doctoral dissertation, University of Georgia.

Tucker, A. (1981). Recommendations for a General Mathematical Sciences Program. Report of the Committee on the Undergraduate Program in Mathematics Panel on a General Mathematical Sciences Program. Washington, D.C.: Mathematical Association of America. 
Tucker, A. (2002). Applied Combinatorics (4th ed.). New York: John Wiley \& Sons.

Vinner, S. (1997). The pseudo-conceptual and the pseudo-analytical thought process in mathematical learning. Educational Studies in Mathematics, 34, 97-129.

Warner, L. (2008). How do students' behaviors relate to the growth of their mathematical ideas? Journal of Mathematical Behavior, 27 (3), 206-227.

White, R. (2009). Encrypted objects and decryption processes: problem-solving with functions in a learning environment based on cryptography. Educational Studies in Mathematics, 72, 17-37. 


\section{Appendix A - Counting survey used in student selection}

Name:

Please circle the classes you have taken, and, if applicable, please write the term and/or year in which you took the class. If you have taken an equivalent class at a different school, please indicate that as well. Thank you!

STAT 243 (Intro to Stats I)

STAT 451 (Intro to Math Stats)

MATH 356 (Discrete Math)

MATH 449/549 (Advanced Number Theory)
STAT 244 (Intro to Stats II)

MATH 346 (Number Theory)

MATH 481/581 (Probability for Teachers)

MATH 487/587 (Topics in Combinatorial Analysis)

Have you taken any classes not on the list that included combinatorics? If so, please list them.

Please list your major(s):

Approximately what year are you? (freshman, sophomore, junior, senior)

Have you previously earned a bachelor's degree? If so, what was your major?

1. You have 5 different Spanish books, 6 different French books, and 8 different German books. How many ways are there to pick a pair of books that are not in the same language? Show your work, and provide any explanation or insight you think might be helpful. 
2. Suppose there are 10 male professors and 15 female professors in the mathematics department at State University.

a. How many different committees could be formed that consist of five men and five women?

b. How many different committees could be formed that have six people, where at least three of them are women? 
3. At Euler's Donut Shop, there are eight different kinds of donuts to choose from (maple frosted, glazed, chocolate frosted, etc.). Suppose you want to fill a box with a dozen donuts to bring back to your friends. In how many different ways could you do this? For example, you could have half glazed and half chocolate.

4. Have you seen any of the following notations below? If so, please explain what you take them to mean.

${ }_{9} C_{4}$

${ }_{4} P_{2}$

$n !$ 
5. The math problems you have just done are called "counting problems." Using the options below, what best characterizes your feelings about counting problems? Please circle all that apply.

I do not know enough about counting problems to have an opinion.

I do not like to solve counting problems.

I am intimidated by counting problems.

Counting problems seem difficult, but I would like to learn more about them.

I find counting problems to be challenging but rewarding.

I love counting problems!

Other:

6. Using the options below, what best characterizes your prior experience with counting problems? Please circle all that apply.

I have never seen anything like these problems before.

I have seen similar problems before, but I never learned (or was taught) how to solve them.

I have seen similar problems before, and I learned (or was taught) how to solve them, but I forgot.

I have seen problems like these before, and I feel confident that I can solve them.

Other:

7. Using the scale below, what best characterizes your level of interest in being selected as an interviewee? Please circle all that apply.

Please do not contact me!

I am ambivalent.

I am a little nervous to commit because I don't know much counting.

I am somewhat interested.

I am quite interested.

Please sign me up! 


\section{Appendix B - Coding Schemes}

In this Appendix I include all of the schedules of Coding Scheme 1, all of the Schedules of Coding Scheme 2, as well as the rationales behind their inclusion. I also provide numerical data for the double coding that occurred.

\section{B.1 - Coding Scheme 1}

\section{B.1.1 - Coding Scheme 1 Front Page}

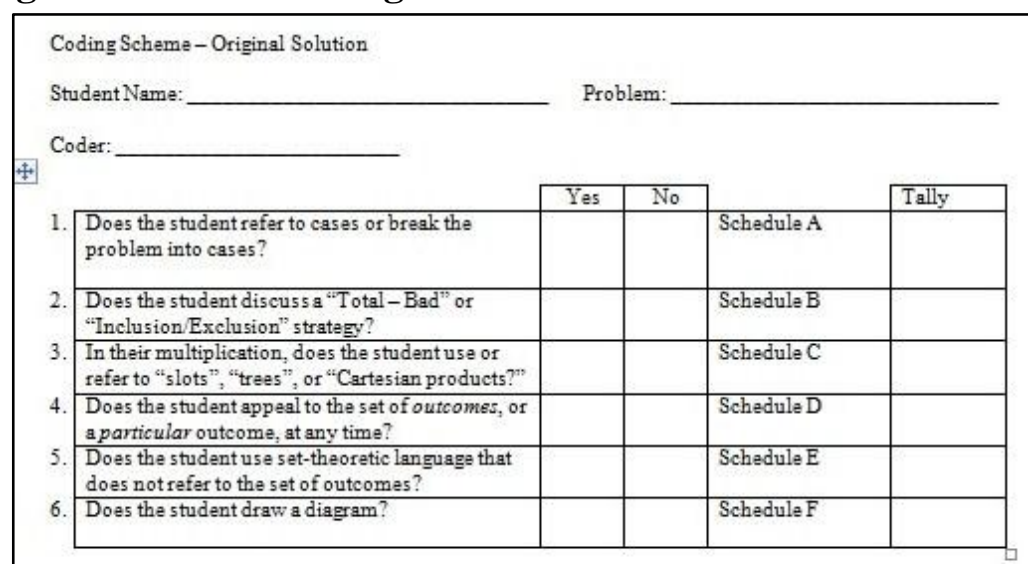

Please check which of the following language students used (or activity they engaged in) in their work on the problem. Please check all that apply.

\begin{tabular}{|l|l|l|}
\hline Check & & Lines \\
\hline & Cases & \\
\hline & Partition & \\
\hline & Categories & \\
\hline & Scenario5 & \\
\hline & Types & \\
\hline & Possibilities & \\
\hline & Drew a Venn Diagram & \\
\hline & Sum/Addition & \\
\hline & Probability & \\
\hline & Dependent & \\
\hline & Independent & \\
\hline & Conditional & \\
\hline & Unconditional & \\
\hline & & \\
\hline & & \\
\hline & & \\
\hline & & \\
\hline
\end{tabular}

\begin{tabular}{|l|l|l|}
\hline Check & & Lines \\
\hline & Overlap & \\
\hline & Complement & \\
\hline & Disjoint & \\
\hline & Union & \\
\hline & Intersection & \\
\hline & Gestured suggesting cases & \\
\hline & Restriction & \\
\hline & Outcome & \\
\hline & Sets & \\
\hline & Collections & \\
\hline & Events & \\
\hline & Ways & \\
\hline & Groups & \\
\hline & & \\
\hline & & \\
\hline & & \\
\hline & & \\
\hline
\end{tabular}

Figure B.1 
The front page of Coding Scheme 1 includes an index of the possible schedules that the coder would potentially fill out. This allowed for the coder to identify which schedules he or she should proceed to answer in detail. If the student never drew a diagram, for example, there would be no need to fill out a Schedule F. Also on this schedule we see a list of set-oriented language that students might have used throughout their work on a task. The coder could take note of what terms came up for students, and there was also room to include some other words as well. I included this because I wanted to get a sense of the kind of set-oriented language students used as they solved these problems.

The emphasis on the particular schedules listed on the front page stems from my hypotheses from Stage 1 and from themes that emerged through my analysis of the data (as shown in Figure B.1 above). Specific items like case breakdowns, total-minus-bad, a consideration of particular outcomes, and "slots" language were all prominent phenomena that I had conjectured might have had some relationship with students' setoriented thinking. Thus, the schedules discussed in Coding Scheme 1 were not simply randomly chosen, they were rooted in my three previous careful passes through the data and my consideration of particular ways in which set-oriented thinking arose for students. In the paragraphs that follow I describe the schedules and some of the rationale for their design.

\section{B.1.2 - Coding Scheme 1 Schedule A}




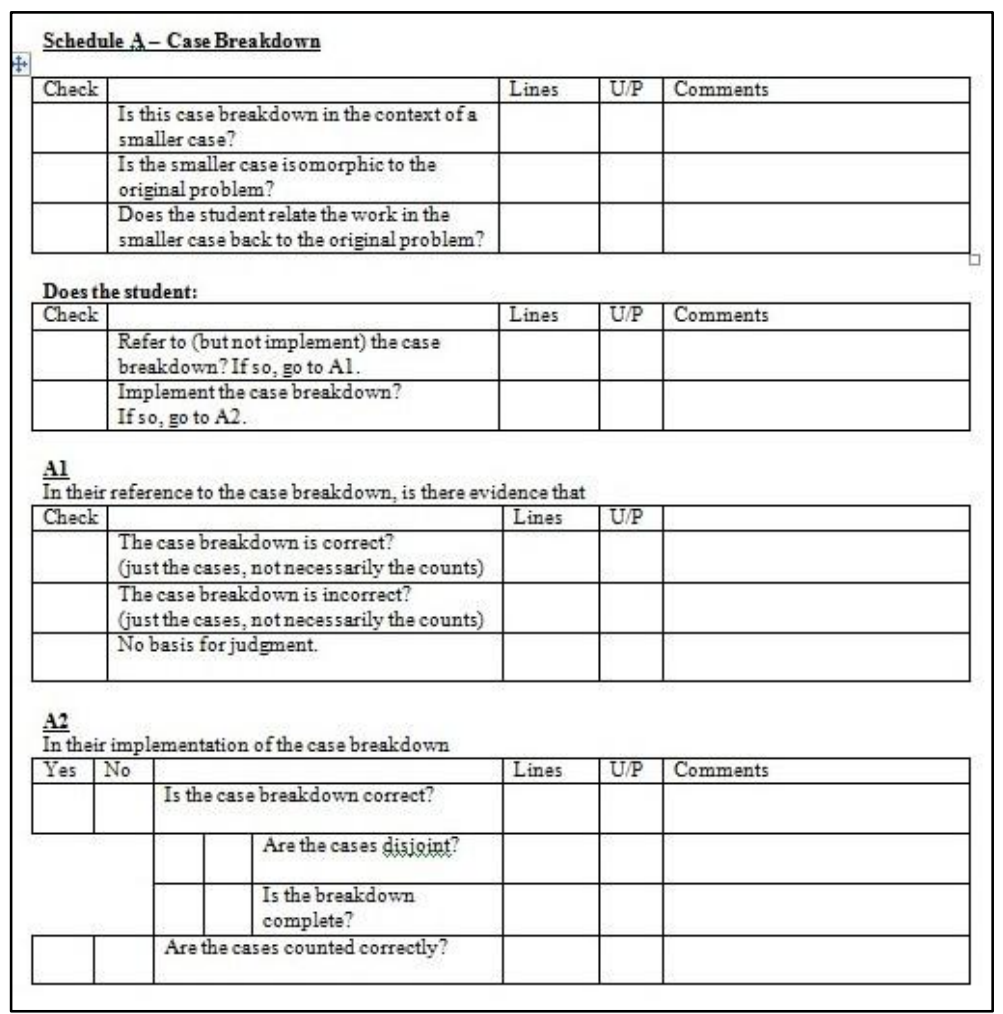

Figure B.2

Schedule A was designed to answer questions about case breakdowns. The first three lines in Schedule A were included to address case breakdowns within small cases, as opposed to having a separate schedule for smaller cases. While many students implemented case breakdowns, I knew that there had been times in which students mentioned but did not execute a case breakdown, and I wanted to distinguish between these two ways in which a case breakdown might have arisen for a student. I felt that even a mention of a case breakdown could signify some set-oriented thinking, and I did not want to focus only on the implementation of a case breakdown. A2 allowed me to get a sense of when students' case breakdowns were correct. In particular, mathematically the important elements of a case breakdown are that they partition the set of outcomes 
into disjoint subsets whose union is the entire set of outcomes, and I wanted to consider that as separate from how they then actually counted the cases. A2 thus allowed me to identify situations in which a student got a problem wrong, but in which they had still correctly determined an appropriate way to break the problem into cases. Also, in filling out the schedule, the coders were asked to use their judgment about the kind of language a student used. A student might never have said "disjoint," but the box might still have been appropriately checked.

\section{B.1.3 - Coding Scheme 1 Schedule B}

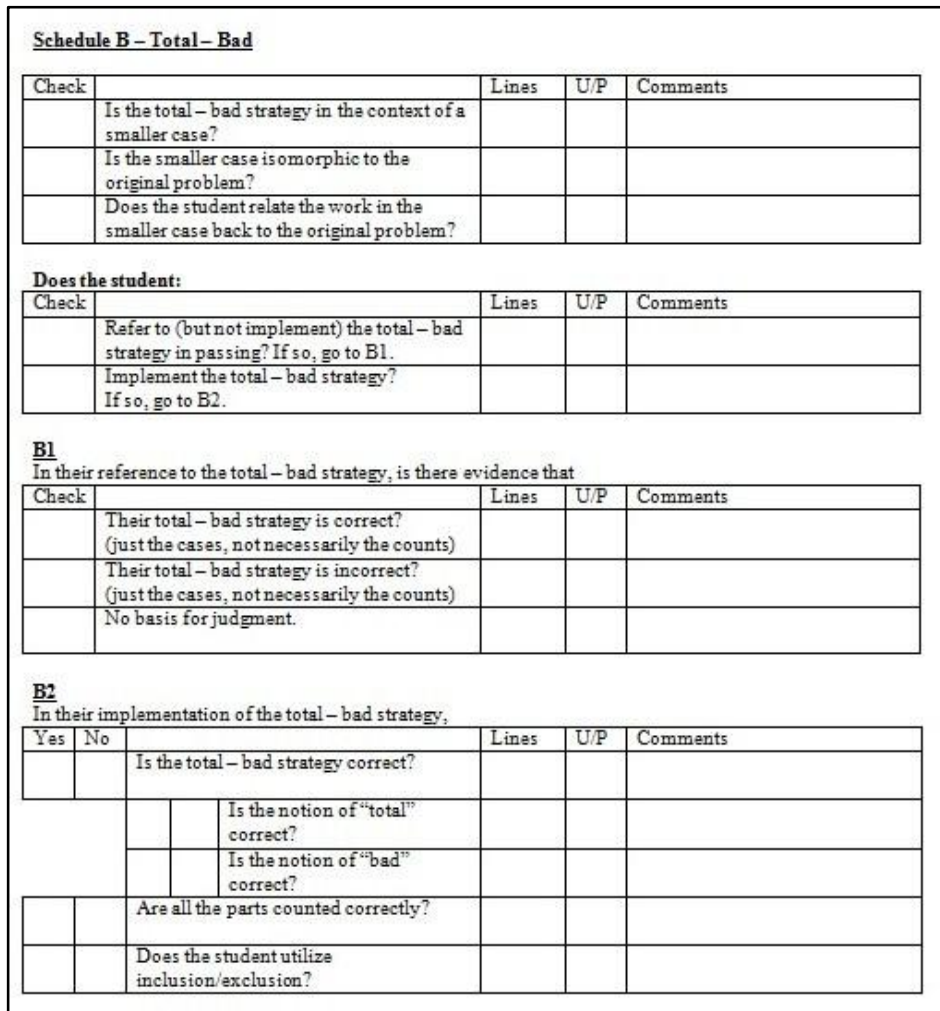

Figure B.3 
Similarly to Schedule A, In Schedule B the first lines had to do with smaller cases, and I made a distinction between students' reference to and implementation of the total-minusbad strategy. I also made a distinction between how students articulated the total-minusbad relationship and how they actually counted the total and the bad. I knew that students could potentially correctly organize the set of outcomes by considering total-minus-bad, but that they might have made an error as they counted either the total or the bad. If a student was going to get a problem wrong when they used a total-minus-bad strategy, I wanted to know if it was because they incorrectly considered total-minus-bad (which could say something about their set-oriented thinking, perhaps) or if they correctly implemented total-minus-bad but incorrectly counted the parts. Finally, inclusion/exclusion was included because it is a more explicitly set-theoretic version of total-minus-bad, and I was curious how and whether the principle of inclusion/exclusion would arise for students.

\section{B.1.4 - Coding Scheme 1 Schedule C}




\begin{tabular}{|c|c|c|c|c|c|}
\hline \multicolumn{6}{|c|}{ Schedule C-Slots, Trees, Cartesian Productst } \\
\hline \multirow[t]{4}{*}{\begin{tabular}{|l|l|l|l} 
Check \\
\end{tabular}} & \multirow{2}{*}{\multicolumn{2}{|c|}{$\begin{array}{l}\text { Is the work described below in the context of a } \\
\text { smaller case? }\end{array}$}} & Linas & $\mathrm{U} / \mathrm{P}$ & Comments \\
\hline & & & & & \\
\hline & \multicolumn{2}{|c|}{$\begin{array}{l}\text { Is the smaller case is omorphic to the original } \\
\text { problem? }\end{array}$} & & & \\
\hline & \multicolumn{2}{|c|}{$\begin{array}{l}\text { Does the student relate the work in the smaller } \\
\text { case back to the original problem? }\end{array}$} & & & \\
\hline \multicolumn{6}{|c|}{ Does the student: } \\
\hline \multirow[t]{3}{*}{ 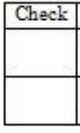 } & \multirow{2}{*}{\multicolumn{2}{|c|}{ Use or refer to "slots"? If so, go to $\mathrm{Cl}$. }} & Lines & U/P & Comments \\
\hline & & & & & \\
\hline & \multicolumn{2}{|c|}{$\begin{array}{l}\text { Use or refer to "trees" or "Cartesian product"? } \\
\text { If } 50, \text { go to } \mathrm{C} 2 \text {. }\end{array}$} & & & \\
\hline \\
\hline \multirow[t]{8}{*}{\begin{tabular}{|l|l} 
Yes \\
\end{tabular}} & \multirow[t]{3}{*}{ No } & & Lines & $\mathrm{U} / \mathrm{P}$ & Comments \\
\hline & & $\begin{array}{l}\text { Does the student use or refer to } \\
\text { "pos sibilities" for what goes in the slots? }\end{array}$ & & & \\
\hline & & \begin{tabular}{|l|l} 
Are the possibilities correct? \\
\end{tabular} & & & \\
\hline & & $\begin{array}{l}\text { Does the student use or refer to the } \\
\text { "placement" of pos sibilities? }\end{array}$ & & & \\
\hline & & \begin{tabular}{l|l} 
Are the placements correct?
\end{tabular} & & & \\
\hline & & Does the student choose locations? & & & \\
\hline & & $\begin{array}{l}\text { Does the student use binomial } \\
\text { coefficients? }\end{array}$ & & & \\
\hline & & $\begin{array}{l}\text { Does the student use some } \\
\text { other method? }\end{array}$ & & & \\
\hline \multicolumn{6}{|l|}{$\frac{C_{2}}{V}$} \\
\hline \multirow[t]{5}{*}{$\begin{array}{ll}\text { Yes } \\
\text { Yes }\end{array}$} & No & & Linas & $\mathrm{U} / \mathrm{P}$ & Comments \\
\hline & & $\begin{array}{l}\text { Does the student use or refer to the } \\
\text { multiplication as "treas"? }\end{array}$ & & & \\
\hline & & \begin{tabular}{l|l} 
Are they correct in their \\
discussion?
\end{tabular} & & & \\
\hline & & $\begin{array}{l}\text { Does the student use or refer to the } \\
\text { multiplication as a "Cartesian Product"? }\end{array}$ & & & \\
\hline & & $\begin{array}{l}\text { Are they correct in their } \\
\text { discussion? }\end{array}$ & & & \\
\hline
\end{tabular}

Figure B.4

Mathematically, Schedule C essentially allowed for a description of ways in which the multiplication principle arose for students. While the "slots" language had not been one of the indicators of set-oriented thinking I had been noticing in my examination of the data thus far (unlike cases, total-minus-bad, or appealing to particular outcomes), it was clear that it was a very prominent way for students to talk about their counting activity. Indeed, language involving slots (and choices for what to put in slots) arose quite often in the data. And, while I had not seriously thought about how slots might have been related to set-oriented thinking, I did feel that perhaps slots could be related to sets of outcomes in some way, and I wanted to keep open the possibility of examining students' slots 
language more closely. Because the slots language has to do with students' ideas about the multiplication principle, I decided to include in the schedule another way that multiplication arose for students that could be related to set-oriented thinking - namely tree diagrams and Cartesian products. So this schedule was designed to provide a way to record instances in which students used or referred to slots, trees, or Cartesian products in their work on these problems. And, to reiterate, this was one schedule that I made without knowing for sure how closely it would relate to my findings on set-oriented thinking.

\section{B.1.5 - Coding Scheme 1 Schedule D}

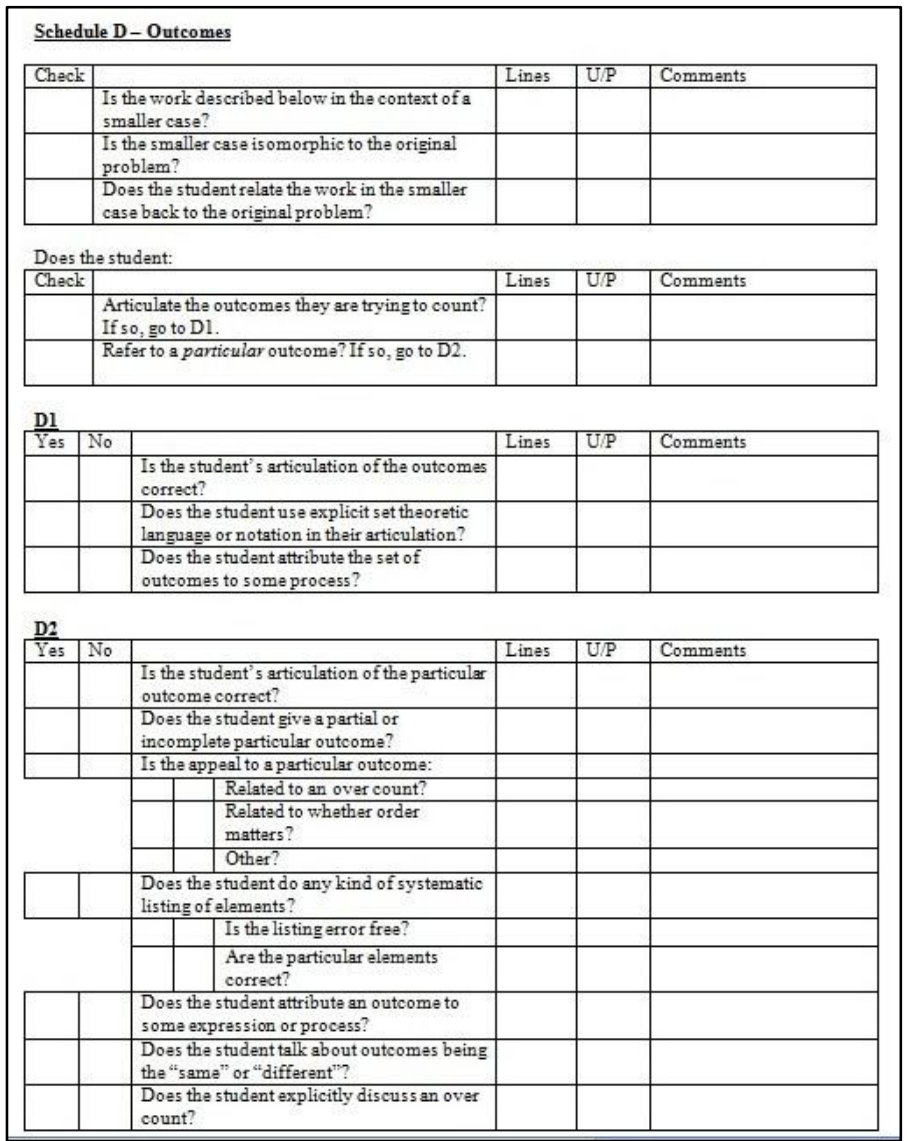


Figure B.5

Students' appeals to particular outcomes had been a recurrent aspect of set-oriented thinking that seemed to be quite promising. I wanted to include it in my analysis, and thus I created Schedule D to record students' references to the set of outcomes. The first three lines again get at whether students referred to outcomes in the context of a smaller problem. I included D1 to allow for a reference to the entire set of outcomes that might not have involved a particular outcome, while D2 focused on students' appeals to particular outcomes. D2 was another example of the ways in which my previous analysis affected my development of Coding Scheme 1. In particular, in my analysis to that point I had seen students refer to a particular outcome primarily to determine an overcount or to determine whether order mattered, and I thus included those as specific questions on the schedule. I also wanted to keep open the possibility that some other motivation for appealing to particular outcomes might arise, and so I included the "other" option as well. I was also interested in students' systematic listing of elements, and the language of "same" and "different" had also come up as something that seemed to be a relevant part of students' set-oriented thinking and as being related to the coordination of sets and processes. The importance of the coordination of sets and process was just emerging as Coding Scheme 1 was developed, and so it was not explicitly mentioned in any of these schedules. However, while Coding Scheme 1 does not have something particularly related to the coordination of sets and processes, this Schedule D gets at some of those ideas. 


\section{B.1.6 - Coding Scheme 1 Schedule E}

\begin{tabular}{|c|c|c|c|c|c|c|c|}
\hline \multicolumn{8}{|c|}{ Schedule E- "Set" language } \\
\hline \multirow[t]{4}{*}{ Check } & \multirow{2}{*}{\multicolumn{3}{|c|}{$\begin{array}{l}\text { Is the work described below in the context } \\
\text { of a smaller case? }\end{array}$}} & Lines & $\mathrm{U} / \mathrm{P}$ & C & aments \\
\hline & & & & & & & \\
\hline & \multicolumn{3}{|c|}{$\begin{array}{l}\text { Is the smaller case is omorphic to the } \\
\text { original problem? }\end{array}$} & & & & \\
\hline & \multicolumn{3}{|c|}{$\begin{array}{l}\text { Does the student relate the work in the } \\
\text { smaller case back to the original problem? }\end{array}$} & & & & \\
\hline \multicolumn{8}{|c|}{ In their usage of set-theoretic language, } \\
\hline \multirow[t]{4}{*}{\begin{tabular}{|l|l|} 
Yes \\
\end{tabular}} & \multirow[t]{3}{*}{ No } & & & & Lines & $\mathrm{U} / \mathrm{P}$ & Comments \\
\hline & & \multicolumn{3}{|c|}{$\begin{array}{l}\text { Is the student referring to the set of } \\
\text { choices/options they have for a particular } \\
\text { situation or event? }\end{array}$} & & & \\
\hline & & & $\begin{array}{l}\text { Is their articulation of the set } \\
\text { correct? }\end{array}$ & & & & \\
\hline & & $\begin{array}{l}\text { Is the s: } \\
\text { so, ples }\end{array}$ & $\begin{array}{l}\text { udent referring to some other set? } \\
\text { se elaborate. }\end{array}$ & & & & \\
\hline
\end{tabular}

Figure B.6

Schedule E was designed to get at any other set-theoretic language that students might have used but that was not accounted for in the other schedules. In particular, I wanted to be able to note how else set-theoretic language might arise that was not particularly related to the set of outcomes. The first three lines again allowed for a smaller case. In what I had seen of the data to that point, students had referred to the set options they had to choose from for some given event, and this was therefore included as a specific question, and I also wanted to allow for space for other ways in which set theoretic language that was not clearly about the set of outcomes might arise.

\section{B.1.7 - Coding Scheme - Schedule F}




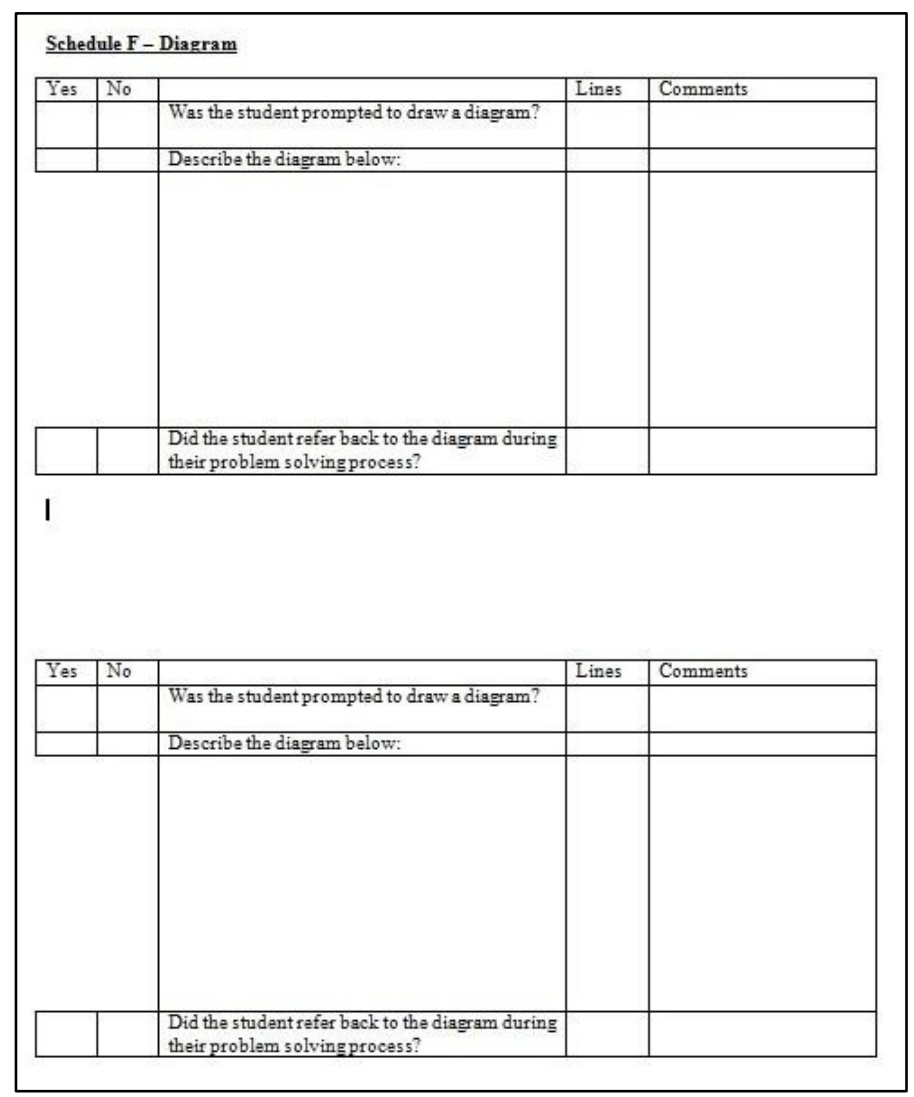

Figure B.7

Schedule F allowed for the coders to draw any diagrams the students might have written and to make comments about it. It also included questions about whether the student was prompted to draw a diagram and whether the student referred back to the diagram during the problem solving process. When I wrote the Coding Scheme, I was unsure how much I would end up using the analysis of the diagrams. However, diagrams seemed to be an important part of the students' counting activity, and especially given that they were relatively easy to code and identify, it would have been a missed opportunity not to pay attention to them in the coding process. 
Lockwood Dissertation - 427

\section{B.2 - Coding Scheme 2}

\section{B.2.1 - Coding Scheme 2 Front Page}

\section{Coding Scheme - Revisiting an Alternative Expressionl}

Student Name:

Problem:

Coder:

What was the student's original answer to the problem?

What is the new solution the student was given?

\begin{tabular}{|c|c|c|c|c|c|}
\hline & & Yes & No & & Tally \\
\hline 1. & $\begin{array}{l}\text { Does the student make sense of an alternative } \\
\text { solution? }\end{array}$ & & & Schedule A & \\
\hline 2. & Does the student compare equivalent solutions? & & & Schedule B & \\
\hline 3. & Does the student compare different solutions? & & & Schedule B & \\
\hline 4. & Does the student coordinate sets and proces ses? & & & Schedule C & \\
\hline 6. & Does the student draw a diagram? & & & Schedule D & \\
\hline 7. & Does the student utilize a smaller case? & & & Schedule E & \\
\hline
\end{tabular}

\begin{tabular}{|l|l|l|}
\hline Check & & Lines \\
\hline & Cases & \\
\hline & Partition & \\
\hline & Categories & \\
\hline & Scenarios & \\
\hline & Types & \\
\hline & Possibilities & \\
\hline & Drew a Venn Diagram & \\
\hline & Sum/Addition & \\
\hline & Probability & \\
\hline & Dependent & \\
\hline & Independent & \\
\hline & Conditional & \\
\hline & Unconditional & \\
\hline & & \\
\hline & & \\
\hline
\end{tabular}

\begin{tabular}{|l|l|l|}
\hline Check & & Lines \\
\hline & Overlap & \\
\hline & Complement & \\
\hline & Disjoint & \\
\hline & Union & \\
\hline & Intersection & \\
\hline & Gestured suggesting cases & \\
\hline & Restriction & \\
\hline & Outcome & \\
\hline & Sets & \\
\hline & Collections & \\
\hline & Events & \\
\hline & Ways & \\
\hline & Groups & \\
\hline & & \\
\hline & & \\
\hline
\end{tabular}

Figure B.8 
As was the case with Coding Scheme 1, I wanted a Front Page that could indicate which particular schedules to fill out. I also included the set-oriented language that students used on the problem, and I provided space to write down what the students' original response to a particular question was, as well as the expression to which they were comparing their answer. In some cases, students evaluated multiple alternative answers to a given problem, and it was my convention to use separate Front Pages (and corresponding schedules) for each of the alternative expressions they evaluated.

\section{B.2.2 - Coding Scheme 2 Schedule A}

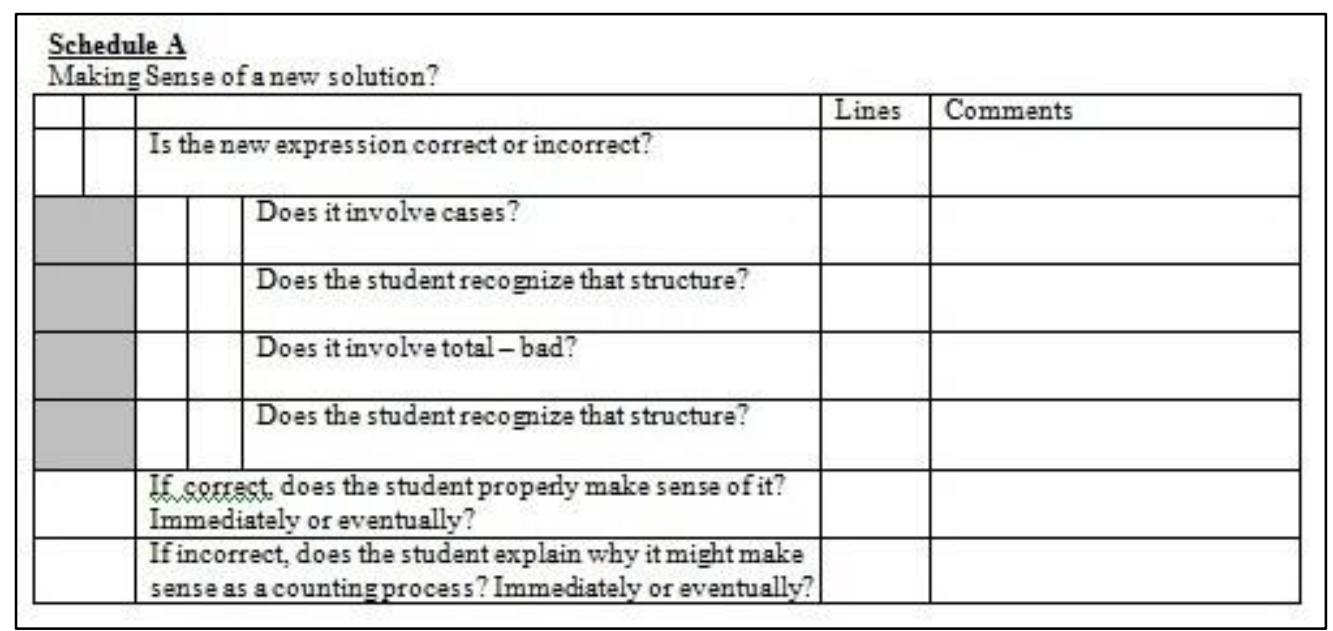

Figure B.9

The alternative answers ranged in their form and in their correctness, and Schedule A was designed to give a way to provide data on the alternative expressions. That is, I wanted to know if an alternative expression involved a form that might have already been related to set-oriented thinking (such as involving cases or total-minus-bad), and I also wanted to know whether or not the alternative answer was correct. By "making sense of" a new 
solution, I meant that a student could explain the process represented by a given expression.

\section{B.2.3 - Coding Scheme 2 Schedule B}

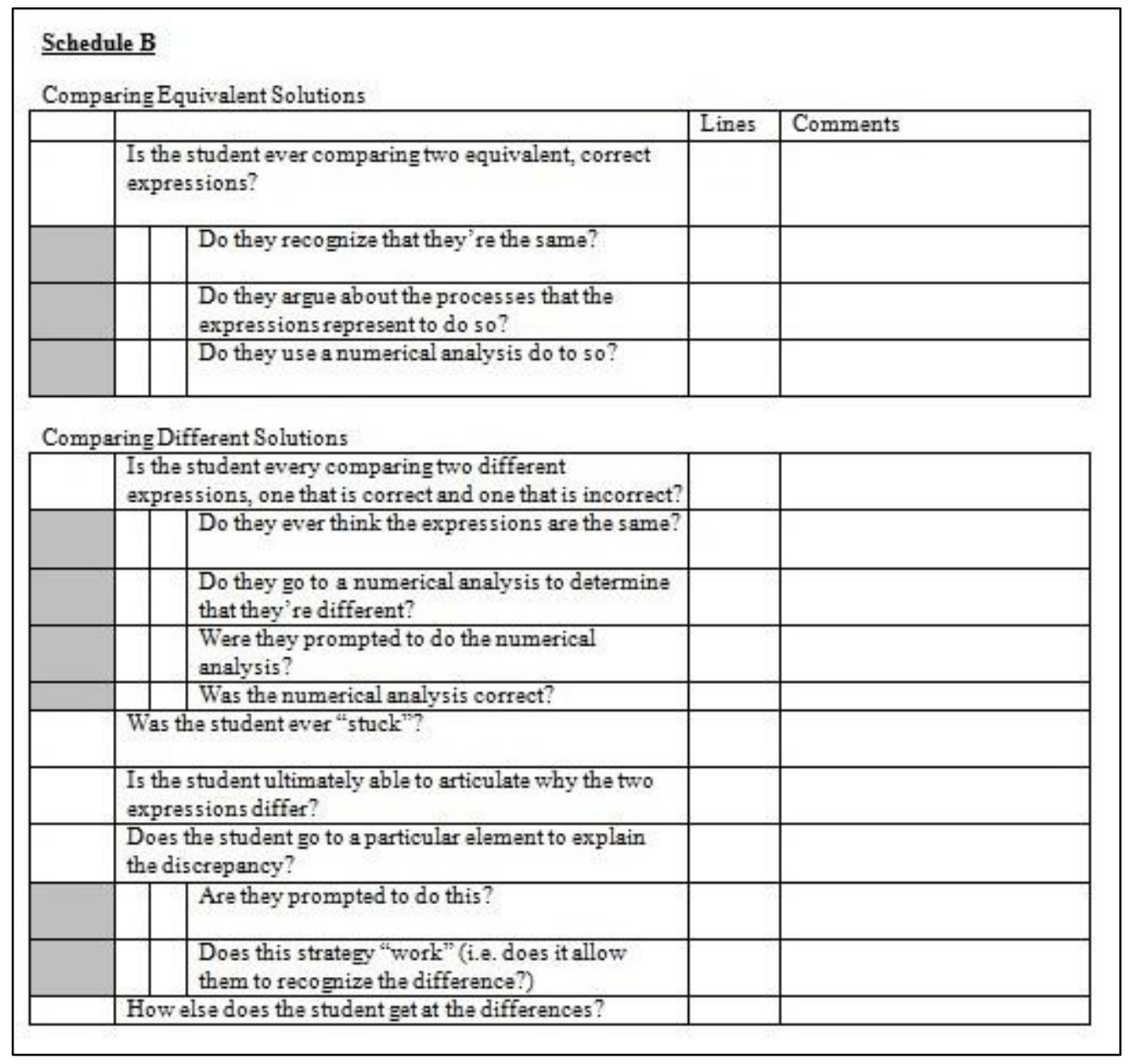

Figure B.10

I included Schedule B because I had specifically designed the study so that students would be put in situations in which they would evaluate seemingly-similar counting processes. I wanted to see if students would consider elements of the set of outcomes in 
order to identify or verify a difference between two processes that might otherwise have seemed the same. So this schedule was designed to get at those relevant parts of that aspect of the design of the study.

I also wanted to differentiate between instances in which students compared equivalent answers and non-equivalent answers, and the two parts of Schedule B allowed for this. I had noticed in my experience in the data that there were times in which students thought two different expressions were actually the same (based on their evaluation of the counting processes), and I allowed for the recording of instances in which that had occurred. I also wanted to get a sense of times in which students were unsure of how to proceed on a problem, and because in many of the problems students had appealed to a particular element of the set of outcomes that had been overcounted, I included that as a specific aspect of the schedule.

\section{B.2.4 - Coding Scheme 2 Schedule C}




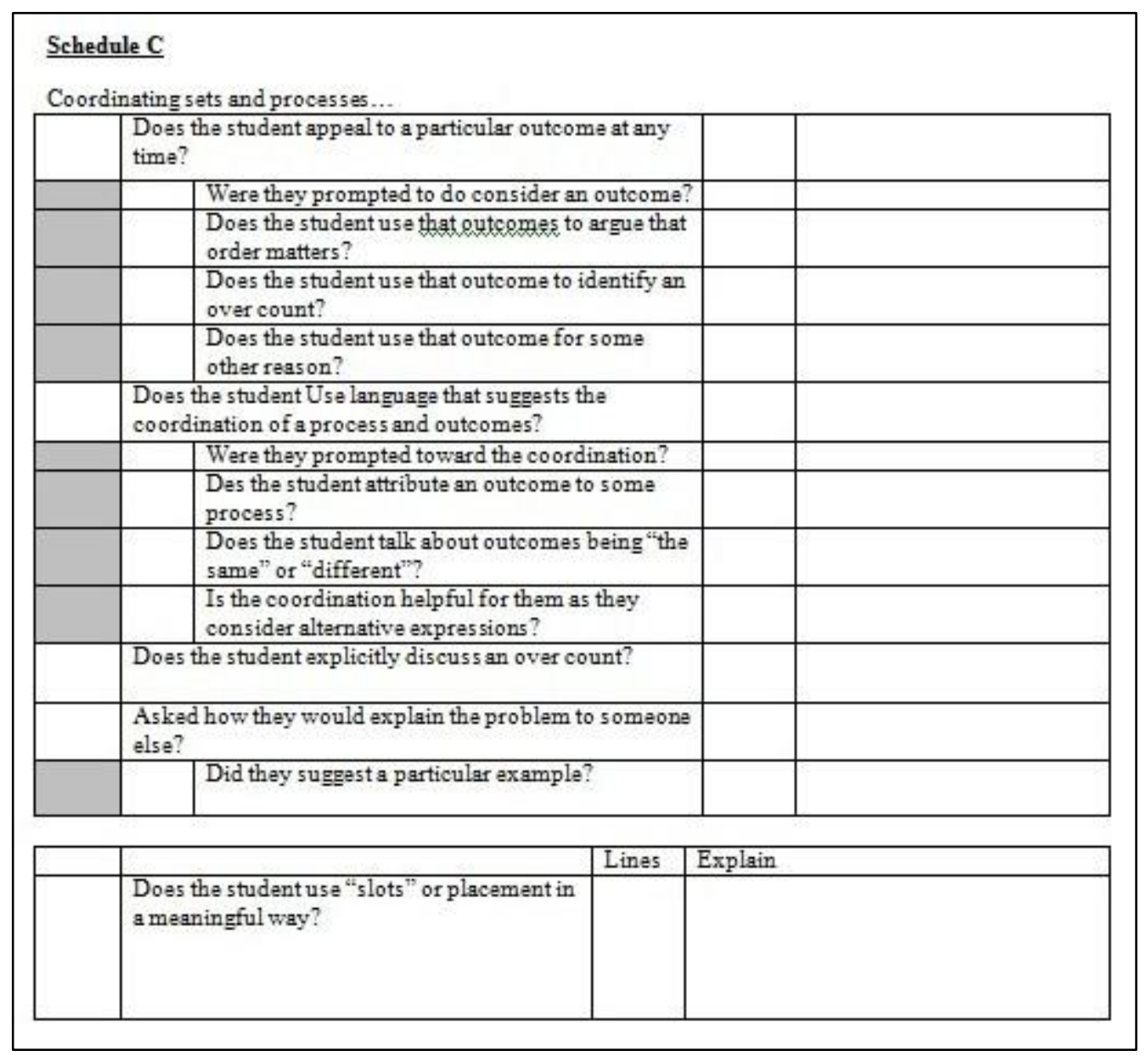

\section{Figure B.11}

At the point at which Coding Scheme 2 was being developed, I had in my analysis begun to suspect that the coordination of sets and processes was important. At this point, what I meant by "coordinating sets and processes" in this schedule was that students showed some sign that they understood that a process could generate some set of outcomes, or that some set of outcomes could be counted by some counting process. I had also noticed that many of the times when students did this, they had identified some particular outcome that a process had yielded. So the questions developed in Schedule C were 
designed to further explore this phenomenon of coordinating sets and processes. Furthermore, in my experience with the data there had seemed to be cases in which students used language to talk about particular elements being the "same" or being “different," and I wanted to record instances of this language as well.

\section{B.2.5 - Coding Scheme 2 Schedule D}

\begin{tabular}{|l}
\hline \multicolumn{2}{|l|}{ Schedule D } \\
Diagram \\
\begin{tabular}{|l|l|l|l|l|}
\hline Yes & No & \multicolumn{1}{l|}{} & \\
\hline & & $\begin{array}{l}\text { Did the student draw or refer to a } \\
\text { new diagram? }\end{array}$ & Lines & Comments \\
\hline & & Describe the diagram below: & & \\
\hline & & & \\
\hline & & $\begin{array}{l}\text { Was the student prompted to draw } \\
\text { the diagram? }\end{array}$ & & \\
$\begin{array}{l}\text { Was the diagram useful in the } \\
\text { students' work with an alternative } \\
\text { problem? }\end{array}$ & & \\
\hline
\end{tabular} \\
\hline
\end{tabular}

Figure B.12

Schedule D showed instances of students' diagrams. I took note of whether the diagram was prompted and whether it appeared to be useful for students as they considered the alternative answer. This was included because at times it seemed as though students only drew the diagram to explain their work to me at the end, or they drew it but never made 
another reference to it. I wanted to be able to record those instances in which the diagram was useful for students as they evaluated alternative answers.

\section{B.2.6 - Coding Scheme 2 Schedule E}

\begin{tabular}{|c|c|c|c|c|c|c|c|c|}
\hline \multicolumn{9}{|c|}{$\begin{array}{l}\text { Schedule E } \\
\text { Smaller case }\end{array}$} \\
\hline \multirow[t]{5}{*}{ Yes } & No & \multirow{2}{*}{\multicolumn{2}{|c|}{$\begin{array}{l}\text { Is the smaller problem is omorphic to } \\
\text { the orisinal problem? }\end{array}$}} & Lab & wo & Aetiving & Bencfeal & Cament \\
\hline & & & & & & & & \\
\hline & & \multicolumn{2}{|c|}{$\begin{array}{l}\text { Does the student talk about outcomes } \\
\text { being "the same"? }\end{array}$} & & & & & \\
\hline & & \multicolumn{2}{|c|}{$\begin{array}{l}\text { Does the student attribute an outcome } \\
\text { to the new process? }\end{array}$} & & & & & \\
\hline & & \multicolumn{2}{|c|}{$\begin{array}{l}\text { Does the appeal to a particular } \\
\text { outcome arise as the student: }\end{array}$} & & & & & \\
\hline & & & Determines an over count? & & & & & \\
\hline & & & $\begin{array}{l}\text { Determines whether order } \\
\text { matters? }\end{array}$ & & & & & \\
\hline & & & Other? & & & & & \\
\hline & & \multicolumn{2}{|c|}{$\begin{array}{l}\text { Does the student do any kind of } \\
\text { systematic listing of elements? }\end{array}$} & & & & & \\
\hline & & & Is the listing error free? & & & & & \\
\hline & & & $\begin{array}{l}\text { Are the particular elements } \\
\text { correct? }\end{array}$ & & & & & \\
\hline
\end{tabular}

Figure B.13

As I recorded data from Coding Scheme 1, I realized that there were not many instances in which smaller cases arose. And while I had, in Coding Scheme 1, included questions about smaller cases on each schedule, I opted not to do so for Coding Scheme 2. Therefore, Schedule E was designed to address students' uses of smaller cases as they revisited the problems and evaluated alternative answers. 


\section{B.3 - Results of double-coding of Coding Scheme 1}

When Philip, Brian and I initially coded three episodes that we would compare in order to discuss coding and refine the instrument, we coded Jenny's Cards problem, Matthew's Groups of Student's problem, and Paige's Groups of Students problem. I give the numerical outcomes of the coding of these three schedules below, noting that even before refinement the rate of reliability was fairly high at over $80 \%$.

Generally I counted the number of boxes on a given schedule. If two people coded, . If three people coded, I included. For example, if I was comparing 5 boxes that three people had coded, I calculated the total number of matched boxes out of 15. My rationale for doing this was that because 3 of us coded, I wanted to be able to capture when 2 of us were in agreement. That is, if in a schedule with 5 boxes one person had not checked a box that the other two had, I coded this as $14 / 15$ instead of $4 / 5$. This captures the notion that there was still some agreement among two of the coders. I provide data both for averaging the percentages for each schedule and for computing a percentage based on the total number of boxes in the entire schedule.

When 3 people coded, if one person did not fill out a particular schedule that the other two did, this was captured in the front page tally, which indicated which schedules coders filled out. A 19/21 on the front page indicated, for example, that there were two instances in which one of the people did not fill out the same schedules as the other two. If two of the three people filled out the same schedule but the third did not, I computed the percentage based just on the two people who filled out the schedule. I did this because the 
third person's failure to fill out a schedule was already captured in the front page percentage.

On Jenny's Cards problem, on the front page we matched 19/21 (90.5\%). On Schedule A there was $100 \%$ agreement. Of the 9 boxes to check on the page, exactly the same 5 were checked by all 3 of us, which I score as a 15/15. There was slight discrepancy in the Schedule C. Philip and I had the same 7 boxes filled in; Brian matched on the C2 (3 boxes), but did not have C1 filled out. Thus, that was a total of $18 / 21$ on this page $(86.7 \%)$. The diagrams matched were $5 / 6$, or $(83.3 \%)$. Brian had not made an outcome schedule. Philip and I had, but we differed initially on whether to fill out D1 or D2, and as a result there was an 8/15 agreement (53.3\%). Brian also did not have a Schedule F, but Philip and I had a complete agreement of on the schedule, giving a 6/9 (66.\%) on this schedule. Averaging the percentages of the schedules, we get $80.0 \%$. The total number of boxes that we filled in was 87 , and we matched in 77 , which is $88.5 \%$ agreement.

On Matthew's Groups of Students problem, again on the front page we matched 19/21 (90.5\%). Philip had not drawn a diagram in Schedule F (in our discussion he said that he had simply forgotten), but Brian and I matched on the diagram, giving $4 / 6$ on the diagram $(66.7 \%)$. None of us selected Schedules A, B, or C for this problem. All 3 of us selected Schedule D. Brian and I both selected D2, and of those we agreed on 10/13 boxes $(76.9 \%)$. Philip, on the other hand, selected D1. Brian did not check the Smaller Case Schedule E, but Philip and I agreed in 7/9 (77.8\%). In the "Set language" there was 6/9 agreement (66.7\%). Averaging the percentages of the schedules, we get $80.35 \%$. The 
total number of boxes that we filled in was 58 , and we matched in $4 / 6$, which is $79.3 \%$ agreement. This was a lower number due in part to Philip's forgetting to draw a diagram, which he acknowledged during our meeting. Also, though, there were some discrepancies surrounding the outcome schedule, Schedule D. Given these discrepancies, we discussed the coding of this schedule in particular as we refined the instrument.

On Paige's Groups of Students problem, on the front page we matched 20/21 (95.2\%). Our diagrams matched $8 / 9(88.9 \%)$. In the outcome Schedule D, while we agreed on D2, there were several boxes which did not match (as mentioned above, the outcome schedule was addressed after we discussed the coding of these three episodes). We had 29/39 (74.4\%) of the same boxes checked. We matched 21/27 on the smaller case $(77.8 \%)$. The average percentage was $84.08 \%$. There were 96 total boxes checked in all of the schedules, and we matched on 78 of them, yielding $81.3 \%$.

So, on the three problems that the three of us coded before the instrument had been refined, reliability rating was in the $79-88 \%$ range, depending on how it was calculated. It seemed that the coders matched quite well on the front page, indicating that they were at least filling out the same kinds of schedules and perhaps recognizing the same kind of global behavior. As mentioned above, we discussed the outcome schedule extensively in our debriefing meeting, and we negotiated some of the details of the coding of that schedule.

After making the afore-mentioned changes to Coding Scheme 1, I distributed the remaining 10 for people to code. Brian and I coded Joshua's Groups of student's problem, and we were $100 \%$ reliable (we matched on all the boxes in all the schedules). 
We also coded Makaena's Groups of Students problem, and again we were 100\% reliable. Brian and I also coded Ben's Cards problem and Mike's Apples \& Oranges problem, and the results of comparing these schedules are given below.

On Ben's Cards problem, the front page matched 11/12 (91.7\%). On Schedule A we matched 9/10 (90\%). I included Schedule C, while Brian did not, and on Schedule D we match $13 / 16(81.3 \%)$. Schedule F, the diagram, we matched on our diagram $3 / 3(100 \%)$. The average percentage was $87.7 \%$. There were a total of 41 boxes that we filled in, and we matched in 36 of them, which gave $87.8 \%$

On Mike's Apples and Oranges problem, Brian and I matched 12/12 (100\%) on the front page. We matched 5/5 (100\%) on Schedule A, and we matched 16/16 (100\%) on Schedule D. We matched 3/6 (50\%) on Schedule E, which had to do with students settheoretic language not explicitly related to the set of outcomes. Averaging these percentages yielded $87.5 \%$ as an average. There were a total of 39 total boxes checked, and we matched on 36 of them, yielding a percentage of $92.3 \%$.

Philip and I both coded Paige's Passwords problem. On the front page we matched 11/12 (91.2\%). We matched 18/24 (75\%) on Schedule A, and 6/6 on Schedule B. On Schedule C we matched 20/24 (83.3\%), and on Schedule D we matched 22/36 (61.1\%). On Schedule F we matched 5/6 (83.3\%). Averaging these gives $82.3 \%$. The total number of boxes checked was 108, and we matched on 82 of them, yielding $75.9 \%$. While the percentages are lower on this problem, I suspected that this might be the case even before we coded them. Paige's work on this problem involved quite a few smaller cases, and she worked for over 30 minutes on the problem. Keeping track of and coding her various 
activities in the schedules was particularly difficult given the complexity of her activity, especially when compared with other students' work on other problems.

Brian and Philip also coded Aiden's Passwords problem. On the front page they were 6/6 (100\%). In Schedule A, they agreed on 12/12 (100\%). In Schedule B, they differed by 1, yielding 5/6 (83.3\%). In Schedule C they matched on 8/11 (72.7\%). In Schedule D matched on $12 / 14(85.6 \%)$, and in schedule E they agreed on 5/6 (83.3\%). Averaging these yields $87.48 \%$. There were 55 total boxes checked, and they matched on 48 of them, yielding $87.3 \%$. 


\section{Appendix C - Example of a Content Log}

In this Appendix I provide an example of a content log. Specifically, this was the Content Log I made after Anderson’s first Interview

\begin{tabular}{|c|c|c|}
\hline 0:00 & $\begin{array}{l}\text { He's a computer science major; } \\
\text { enrolled in number theory for the } \\
\text { elective requirement. He took the CS } \\
\text { department discrete; he took stats } 451 \text {; } \\
\text { he's in his } 4^{\text {th }} \text { year. }\end{array}$ & \\
\hline $1: 20$ & $\begin{array}{l}\text { At least } 3 \text { E's problem. He gets that } \\
\text { there would be } 26^{\wedge} 8 \text { in total. He needs } \\
3 \text { E's out of } 8 . \text { He does cases, with } \\
1^{*} 1^{*} 1^{*} 25^{\wedge} 5 \text {, 'because those are set to } \\
\text { be E's.' } \\
\text { He says he'll typically leave it like this. } \\
4: 14-\text { I ask what made him add them } \\
\text { together; he describes that he's thinking } \\
\text { of } 8 \text { different cases, each with exactly } \\
\text { some number of E's. He thinks he's } \\
\text { seen something somewhat similar to it. }\end{array}$ & $\begin{array}{l}\text { He starts out doing a nice job of kind } \\
\text { of talking/thinking out loud. } \\
\text { So this is similar to what Nick did, } \\
\text { with the 1's instead. I'll be very } \\
\text { interested to see if he can reconcile it } \\
\text { in the same way. **and actually, } \\
\text { maybe this would be a good thing to } \\
\text { give other students as well to make } \\
\text { sense of. } \\
\text { Definitely one to revisit! }\end{array}$ \\
\hline $5: 15$ & $\begin{array}{l}\text { Pairs of cards problem - asks if ace is a } \\
\text { face card. He starts by saying that there } \\
\text { are } 12 \text { different ways to pick a face } \\
\text { card at first - and then he crosses it out } \\
\text { and says, "actually let's split this up." } \\
\text { He got the numbers wrong initially } \\
\text { (thought } 4 \text { face cards), so he gets } 8^{*} 13 \\
+4 * 12 \text {, although I wasn't too } \\
\text { concerned about this. He does } \\
\text { articulate, there are } 152 \text { ways to draw } 2 \\
\text { cards from a deck that fit this criteria. } \\
7: 15 \text { I ask about his change to cases. He } \\
\text { uses the over-lap language. Then he } \\
\text { notes his mistake, so he did his math }\end{array}$ & $\begin{array}{l}\text { This is a procedural issue...ha } \\
\text { It's maybe not worth revisiting, but I } \\
\text { wonder if it had been stated as pairs of } \\
\text { cards instead... would that have any } \\
\text { effect. }\end{array}$ \\
\hline
\end{tabular}




\begin{tabular}{|c|c|c|}
\hline & $\begin{array}{l}\text { wrong, and he fixes it. Since the cases } \\
\text { have } 3 \text { common elements. He finds it } \\
\text { simpler; to categorize them, he thinks it } \\
\text { could be done some other way. } \\
\text { I ask about if someone would have } \\
\text { given the answer } 12 * 13 \ldots \text { he explains } \\
\text { it; he says that he's assuming no } \\
\text { replacement. } \\
\text { He describes seeing two possibilities. }\end{array}$ & $\begin{array}{l}\text { There's set language here with } \\
\text { overlap, etc. }\end{array}$ \\
\hline $11: 10$ & $\begin{array}{l}\text { In how many ways can you split a class } \\
\text { of } 20 \text { into four groups of five. Writes } \\
\text { down } 20 \text { students; He writes } \\
20 * 19 * 18^{*} 17 * 16 \text { as the number of } \\
\text { ways to form the first group. He says } \\
\text { that he thinks it would work as a } \\
\text { straight up } 20 \text { !. He explains the product } \\
\text { above; he says there are } 15 \text { students } \\
\text { left, so then there are } 15 \text { ! Ways to make } \\
\text { the remaining } 3 \text { groups. Then he gets to } \\
\text { the } 5 \text { ! And pauses and says it doesn't } \\
\text { work. He says } 5 \text { ! Doesn't work for } 1 \\
\text { groups because then the last group } \\
\text { would have the same members over } \\
\text { and over again. So he resolves this by } \\
\text { doing } 20 \text { ! - } 5 \text { !. } \\
\text { I ask him to describe the solution. But } \\
\text { he realized that all } 5 \text { students would be } \\
\text { in the same group, and he notes that "it } \\
\text { doesn't really matter what order that } \\
\text { fits in the group", the } 5 \text { ! Becomes } \\
\text { moot. He doesn't take away the } 15 \text { ! Or } \\
10 \text { !, because somehow he's thinking } \\
\text { about swapping out previous elements } \\
\text { with the elements of the last group. } \\
\text { I try to clarify - and I ask if he } \\
\text { excluded } 5 \text { ! Because it would count } \\
\text { arrangements. And he notes the order }\end{array}$ & $\begin{array}{l}\text { He does appeal to some solution in } \\
\text { talking about the final group, which is } \\
\text { interesting (maybe set language). } \\
\text { I feel like there's weird stuff going on } \\
\text { here - why is he only paying attention } \\
\text { to the last group?? } \\
\text { This last group swapping language }\end{array}$ \\
\hline
\end{tabular}




\begin{tabular}{|c|c|c|}
\hline & $\begin{array}{l}\text { wouldn't matter in this problem. He's } \\
\text { fixated on the final group only, and he } \\
\text { does still feel okay with recounting the } \\
\text { first group. He explains by naming } \\
\text { them ZYXWV, and he notes that this } \\
\text { could remain as the first group, while } \\
\text { he swaps out elements of the last } 2 \\
\text { groups. } \\
\text { 17:15 - I ask if he's still considering } \\
\text { order in the first group, and this tips } \\
\text { him off that his answer is wrong. He } \\
\text { notices ZXYWV with the rest the same } \\
\text { is the same, and so that makes the first } \\
\text { group needs to be fixed. } \\
\text { He says he needs to re-think the } \\
\text { problem, and he says I've shed light on } \\
\text { something. He thinks he needs to think } \\
\text { about it. He wants to come back to it. }\end{array}$ & makes me think of processes, perhaps. \\
\hline $19: 20$ & $\begin{array}{l}5 \text {-digit numbers. Since it has to be } \\
\text { even, that means the ones digit has to } \\
\text { be } 2,4,6,8,0 \text {. He starts kind of going } \\
\text { the process way, starts with the } 5 \text { and } \\
\text { talks through the rest of the digits. So } \\
\text { he gets } 8^{*} 8^{*} 7 * 6^{*} 5 \text { at first. I ask why he } \\
\text { can do multiplication. He says it's } \\
\text { because he's combining the...he does a } \\
\text { small example to show me. He shows } \\
\text { how if the last digit is a } 2 \text {, then there } \\
\text { are } 8 \text { choices, then he goes to write } 4 \text {, } \\
6,8,0 \text {, and there's something about this } \\
\text { example that shows the } 0 \text { behaves } \\
\text { differently. He says that reminds him of } \\
\text { the case of the } 0 \text { in the back. Since the } \\
0 \text { has to be in the back, he looks at case } \\
\text { where end is not } 0 . \text { So he figures out } \\
\text { the cases and adds them together. } \\
24: 15 \text { I ask him what he'd done and }\end{array}$ & $\begin{array}{l}\text { The fact that he starts w/ the } 5 \text { is } \\
\text { noteworthy - it's kind of unusual to } \\
\text { take that constraint first, in a good } \\
\text { way. } \\
\text { I should say, I had no intention that } \\
\text { asking about multiplication would lead } \\
\text { to this; it was just trying to get him to } \\
\text { keep talking and say something about } \\
\text { multiplication. Turned out it led him to } \\
\text { writing something that showed the } \\
\text { error. }\end{array}$ \\
\hline
\end{tabular}




\begin{tabular}{|c|c|c|}
\hline & $\begin{array}{l}\text { how he recognized the issue. He said he } \\
\text { did it as a simple (explains problem). } \\
\text { When he was explaining, he started to } \\
\text { write out individual numbers. It made } \\
\text { him realize if he had a } 0 \text { in the ones } \\
\text { digit, he didn't have to worry, and so he } \\
\text { broke it into cases. I ask what he saw } \\
\text { there. He says as he's writing } 1 \text { through } \\
\text { 9. If he has } 0 \text {, he can actually use all } 9 \\
\text { numbers. Again I ask him to explain } \\
\text { how cases work. He says he's solving a } \\
\text { sub-problem of numbers ending in } 0 . \\
\text { I ask if he was picturing particular } 5 \text { - } \\
\text { digit problems; he was thinking more } \\
\text { of pieces of info he needed to put } \\
\text { together to generate a number. } \\
27: 55 \text { I ask him how he'd explain to a } \\
\text { student who got } 8 * 8 * 7 * 6 * 5 \text { why their } \\
\text { answer was wrong. He says he'd break } \\
\text { it into a smaller problem, showing how } \\
0 \text { is a different possibility. He says he } \\
\text { could extend it to } 5 \text {-digit numbers, first } \\
\text { to } 3 \text {-digit numbers. And so I guess that } \\
\text { would get at why he needed a separate } \\
\text { case. }\end{array}$ & $\begin{array}{l}\text { It's hard for me to know what to ask } \\
\text { about picturing things; he does kind of } \\
\text { think, broadly, of all numbers ending } \\
\text { in } 0 \text { and all numbers not ending in } 0 \text {, } \\
\text { but then he doesn't really think of } \\
\text { specific ones. I guess I wonder if he } \\
\text { can think of it as being a big set of } \\
\text { even 5-digit numbers, split into those } \\
\text { that end in } 0 \text { and those that don't, but I } \\
\text { don't think I can ask that. }\end{array}$ \\
\hline $30: 45$ & $\begin{array}{l}\text { Test question problem. He decides to } \\
\text { split it into cases again. He draws out } \\
\text { all the possibilities for answering } 2 \text { of } \\
\text { the first } 5 \text { (gets } 10 \text { little solutions). } \\
\text { Then he starts setting up different } \\
\text { scenarios, first as } 2 \text { and } 3 \text {. Then he does } \\
\text { some working to reconcile the } 10 \text {, } \\
\text { figures it's combinations and } \\
\text { permutations. Then he writes out some } \\
\text { more of the cases ( } 3 \text { and } 2,4 \text { and } 1,5 \\
\text { and } 0 \text { ). He sums up the answers and } \\
\text { gets the correct answer of } 226 \text {. }\end{array}$ & Interesting Cartesian product thing! \\
\hline
\end{tabular}




\begin{tabular}{|c|c|c|}
\hline & $\begin{array}{l}\text { I ask why } 10 * 10 \text { gets multiplied, he } \\
\text { explains it as cross product (refers to } \\
\text { databases). } \\
\text { I ask how he decided on c( } 5,2) \text {. He } \\
\text { originally thought permutations (I } \\
\text { generally go to permutations first), and } \\
\text { so he had to reconcile to his diagram; } \\
\text { wait, permutations would separate the } \\
\text { case where I answered the first question } \\
\text { and second question in different orders. } \\
\text { Also, he noticed that he restricted the } \\
\text { cases to smaller problems here. } \\
\text { I asked if a particular answer ran } \\
\text { through his mind, and he explains that } \\
\text { he did think of answering } 2 \text {, so he } \\
\text { thinks that might indicate } \\
\text { combinations...he goes to confirm it } \\
\text { using the formula (from statistics). } \\
\text { Talks about order mattering in } \\
\text { permutations but not in combinations. }\end{array}$ & $\begin{array}{l}\text { This is good - he used the } 1221 \\
\text { example to convince himself that he } \\
\text { needed combinations instead of perms. } \\
\text { Also good! } \\
\text { This is nice...it matched up his } \\
\text { diagram and so he went with that. }\end{array}$ \\
\hline $42: 30$ & $\begin{array}{l}\text { Apples/Oranges problems. He starts to } \\
\text { write down some cases, and in doing so } \\
\text { he creates something of a diagram. He } \\
\text { counts up until } 6 \text { different fruit, and } \\
\text { then he thinks all the rest will be } 6 \text {. } \\
\text { He gets } 68 \text {. I asked how he reasoned } \\
\text { about the } 6 \text {, but he felt better with a } \\
\text { "brute force" method of sorts. He talks } \\
\text { back through the process. It continued } \\
\text { where it was the size plus } 1 . \text { He } \\
\text { explains why it's } 6 \text {. } \\
\text { Then, on the diagram he writes a bunch } \\
\text { of zeros after, and suddenly rethinks } \\
\text { what he's doing. And I say, okay, now }\end{array}$ & $\begin{array}{l}\text { It's interesting; at this point Nancy had } \\
\text { found a pattern and had gone with it, } \\
\text { whereas he keeps going, and it takes a } \\
\text { little longer to see a pattern (and it's a } \\
\text { different pattern). } \\
\text { Something about sets? }\end{array}$ \\
\hline
\end{tabular}




\begin{tabular}{|c|c|c|}
\hline & $\begin{array}{l}\text { how come? He says when we hit size } 9 \text {, } \\
\text { one of them must be an apple, so we } \\
\text { remove the case where there's no } \\
\text { apples. So he fixes his pattern. } \\
\text { He says the pattern makes him think of } \\
\text { the dice problem, because he figures } \\
\text { the possibilities will grow and then } \\
\text { shrink - even though the subsets are of } \\
\text { different sizes, something is the same. } \\
\text { I ask what it was that made him realize } \\
\text { - he said, he's drawing out these O's, } \\
\text { he says wait a second, if I draw out all } \\
\text { the O's, when I get past } 9 \text {, one of the } \\
\text { oranges is already accounted for. So the } \\
\text { list gets smaller. } \\
\text { I asked if visually seeing it tipped him } \\
\text { off, and he said something was in his } \\
\text { mind, but he saw it and he has a limited } \\
\text { number of resources for the other } \\
\text { object, too. Interesting } \\
\text { trapezoid/triangle comparison. }\end{array}$ & $\begin{array}{l}\text { This is actually pretty cool; I like his } \\
\text { explanation. I didn't quite get it during } \\
\text { the interview, but it's making more } \\
\text { sense now. }\end{array}$ \\
\hline $52: 15$ & $\begin{array}{l}\text { Octagon problem. He says, for } \\
\text { simplicity he's going to draw a regular } \\
\text { octagon. He labels it. He writes } 1,2,3 \text { - } \\
8 ; 1,3,3-8 \text {, etc. Then he says, let's not } \\
\text { approach the problem like this. He } \\
\text { decides to break it into types of } \\
\text { triangles. He says he knows there's } \\
\text { going to be } 8 \text {, so he needs to decide } \\
\text { how many different triangles there } \\
\text { could be, but } 126 \text { could be the same as } \\
125 . \text { He knows } 1,2,3 \text { has } 8 \text {, so he's } \\
\text { going to look at another triangle. He } \\
\text { draws things and sees mirror images; } \\
\text { for some he finds } 16 \text { instead of } 8 . \text { He } \\
\text { notes he's not using any numbers past } 5 \\
\text { because he can mirror the triangle. He }\end{array}$ & $\begin{array}{l}\text { I'm not sure how useful this is, } \\
\text { honestly. }\end{array}$ \\
\hline
\end{tabular}




\begin{tabular}{|c|c|}
\hline $\begin{array}{l}\text { feels like there should be more. He gets } \\
16 \text { for } 1,3,6 \text {. So yeah, some talking and } \\
\text { trying to work through this. } \\
\text { He starts writing down } n+1+2, n+1+3 \text {, } \\
\text { etc. He said he thinks he's just } \\
\text { confusing himself; he talks through it } \\
\text { some. Just lots of drawing triangles. He } \\
\text { notes he needs to account for reverse } \\
\text { triangles, too. } \\
\text { He tries canceling out some that he's } \\
\text { already seen. Okay so he get } 64 \\
\text { possibilities. } \\
1: 08: 20 \text { I ask for his strategy. He says } \\
\text { he was confusing himself, so he figured } \\
\text { to just let } n \text { be an arbitrary point on the } \\
\text { octagon; where } n \text { is from } 1 \text { to } 8 \text {, which } \\
\text { is why he multiplied by } 9 \text { at the end. } \\
\text { He says he thinks the problem has to do } \\
\text { with modulo; it's a circle and it cycles } \\
\text { back, so I feel like I'd have to take the } \\
\text { different of } n+1 \text { mod } 8 \text {, or something. }\end{array}$ & $\begin{array}{l}\text { Yeah this kind of goes on longer than } \\
\text { it needs to, I think. }\end{array}$ \\
\hline
\end{tabular}

